\title{
Multi-Component Adsorption of Monoclonal Antibodies and Antibody Aggregates on Cation Exchange Resins
}

\author{
A Dissertation \\ Presented to the faculty of the School of Engineering and Applied Science at the \\ University of Virginia
}

In partial fulfillment of the requirements for the degree of

Doctor of Philosophy in Chemical Engineering

by

Jason M. Reck

December 2017 


\section{Approval Sheet}

This Dissertation is submitted in partial fulfillment of the requirements for the degree of Doctor of Philosophy (Chemical Engineering):

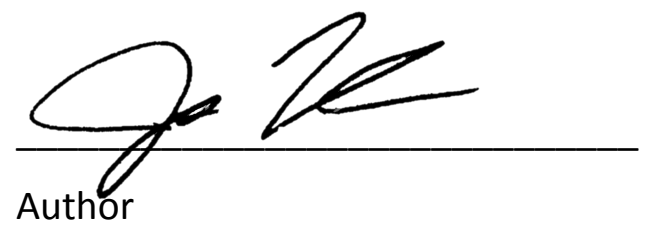

This Dissertation has been read and approved by the examining committee:

Giorgio Carta

Dissertation Advisor

Steven Caliari

Geoffrey Geise

Donald Griffin

Kyle Lampe

Accepted for the School of Engineering and Applied Science:

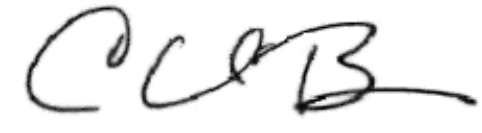

Craig H. Benson, School of

Engineering and Applied Science

December 2017 


\section{Abstract}

The removal of aggregates and, especially, soluble dimers and other moderate mass species, presents a significant challenge during the production of monoclonal antibody (mAb) therapeutics. These aggregate species are a key impurity attribute monitored during downstream processing, due to their lower efficacy and increased likelihood of inciting an immunogenic response. However, the chromatographic separation of these dimers and other soluble species from their monomeric form is often challenging, due in part to low selectivity and in part to slow mass transfer. Further, much of the previous work examining this topic has been largely empirical, relying on large data sets and extensive parameterization to describe the data. The overall goals of this dissertation are to provide a fundamental understanding of the multi-component chromatographic interactions of mAb monomer with its soluble dimer, and to elucidate the underlying mass transfer phenomena governing these interactions.

This work has characterized the behavior of a mAb monomer, as well as that of a processgenerated mAb dimer, for a variety of experimental conditions on Nuvia HR-S, a strong cation exchange resin. The single component behaviors of the proteins were analyzed using a variety of macroscopic measurements (batch equilibrium isotherms, column experiments) and microscopic measurements (confocal laser scanning microscopy) in order to find equilibrium and kinetic parameters. Additionally, light-based techniques (dynamic light scattering, biolayer interferometry) were used to probe the size of the proteins and the kinetics of protein binding. These same techniques were later extended and adapted to investigate the two-component behaviors of mAb monomer and dimer.

A key result of the batch two-component behavior was a strong dependence on the sodium ion concentration on the observed equilibrium and kinetics of the experimental system. At low salt concentrations, the two-component behavior of the monomer and soluble dimer show very little selectivity, a departure from the expected behavior in ion-exchange theory. As the salt concentration is increased to intermediate levels where significant binding is still present, the expected selectivity behavior emerges, with the more strongly bound dimer displacing the 
more weakly bound monomer. These results are attributed to a kinetic resistance to displacement of the monomer by the dimer under conditions where the binding strength is high, a result corroborated by biolayer interferometry measurements of the rate of adsorption and protein-protein surface exchange.

The results of the two-component batch behavior led to the development of a separation scheme for the monomer and dimer via frontal analysis. In this process, a mixture of antibody monomer and dimer is continuously fed to a packed column under conditions where the mixture is favorably bound, resulting in two breakthrough fronts whose monomer and dimer compositions are determined by the two-component equilibrium and kinetics of the system. These experiments were performed at a variety of sodium ion concentrations, resulting in processes that work best at intermediate salt concentrations, again corroborating the observed batch results.

Finally, a numerical model was developed to describe the two-component uptake in column and batch experiments. The purpose of the model is to provide a basis to test various mechanistic models to better understand the observed two-component behavior, as well as provide a means to optimize the experimental conditions required for a successful separation of monomer and dimer species. 


\section{Acknowledgements}

I would first and foremost like to thank my advisor, Prof. Giorgio Carta. Since arriving at the University, you've challenged me to be the best student and researcher I could be. I've learned so much more than what is contained in this dissertation, and you were one of the primary catalysts of that. I sincerely thank you for everything you've done and taught me.

I would like to thank the other members of my committee and the rest of the faculty in Chemical Engineering (past and present) who I've interacted with in my time here. Thank you for the guidance and knowledge you have provided me over the years. The culture of this University and Department was one of the biggest draws that brought me to Charlottesville, and I have never regretted it.

Next, I would like to thank my fellow students, lab mates, and friends who have been with me through grad school. There are too many to name individually, but please know, that much later in life, I will look back fondly on the time I've spent here in Charlottesville and it will primarily be because of all of you.

I would like to thank my parents and sisters for their support. You all had a big part in building the foundation upon which my successes have been built. Thank you for all of the love and encouragement along the way.

Finally, and most importantly, I want to thank Lillian. You've been here with me every step of the way, and I have difficulty expressing how important you've been in helping me get through this journey. I can't wait to start the next chapter of our lives together. 


\section{Table of Contents}

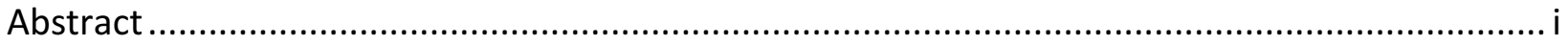

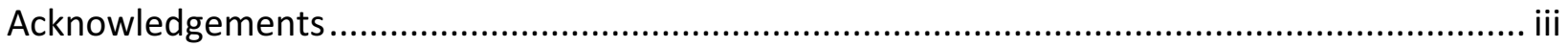

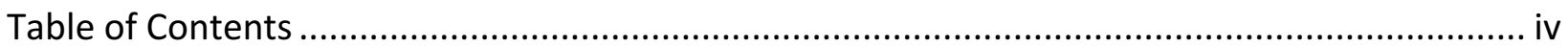

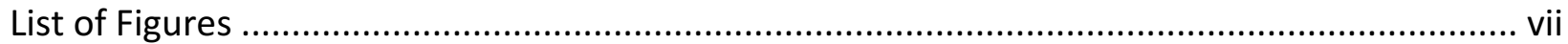

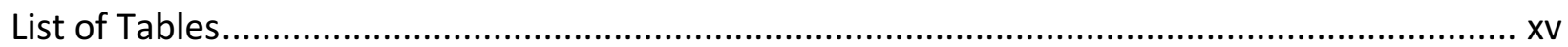

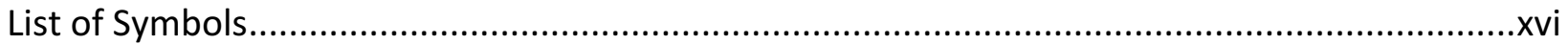

1. Introduction and Background................................................................................... 1

1.1. Monoclonal Antibodies as a Therapeutic Protein ....................................................... 1

1.2. Downstream Processing of Monoclonal Antibodies ................................................ 3

1.3. Aggregate Removal in Downstream Processing ................................................ 6

1.4. Cation Exchange Chromatography ............................................................. 7

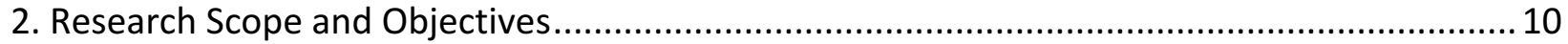

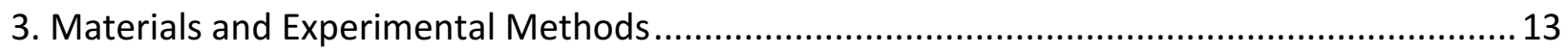

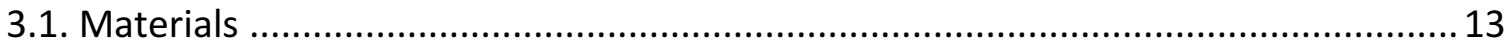

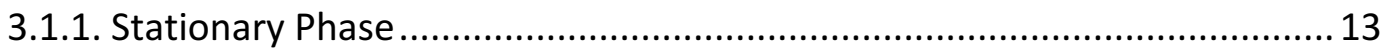

3.1.2. Proteins and Chemicals ...................................................................... 13

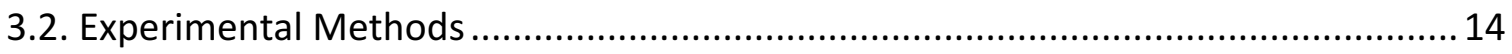

3.2.1. Particle Size Distribution..................................................................... 14

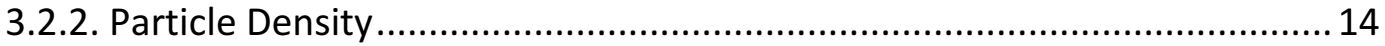

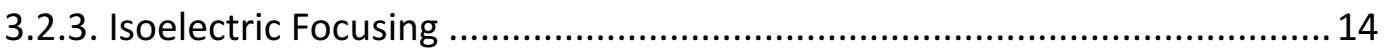

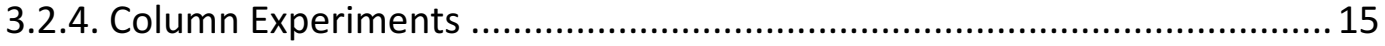

3.2.5. Size Exclusion Chromatography......................................................... 17

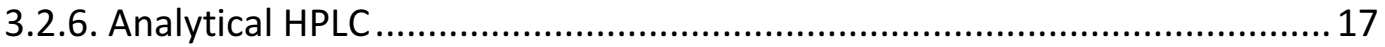

3.2.7. Inverse Size Exclusion Chromatography ................................................ 18

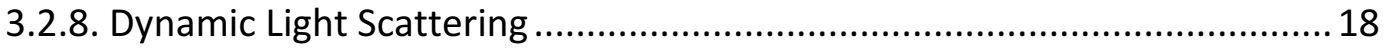

3.2.9. Equilibrium Adsorption Isotherms....................................................... 19

3.2.10. Confocal Laser Scanning Microscopy................................................... 19

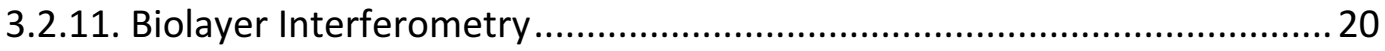




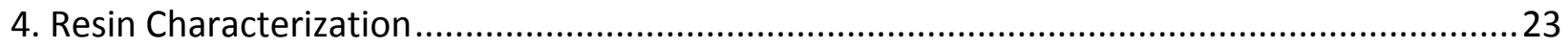

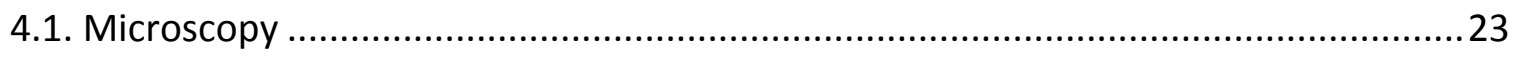

4.2. Inverse Size Exclusion Chromatography ……………...........................................23

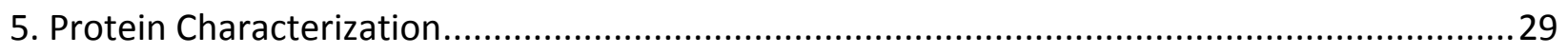

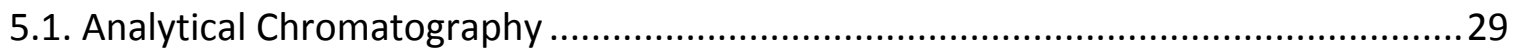

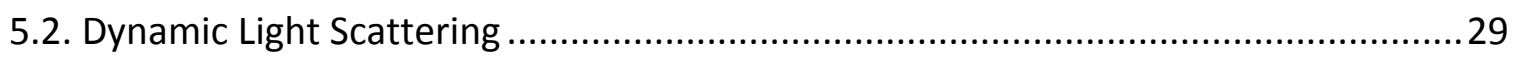

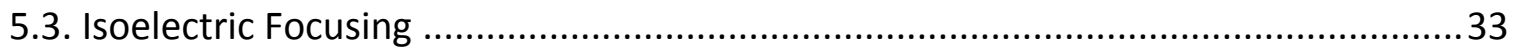

6. Characterization of Single Component Adsorption Equilibrium and Kinetics of Antibody

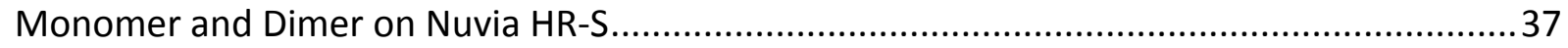

6.1. Retention Behavior at Low Protein Loadings …………........................................37

6.2. Adsorption Isotherms ………….......................................................................... 43

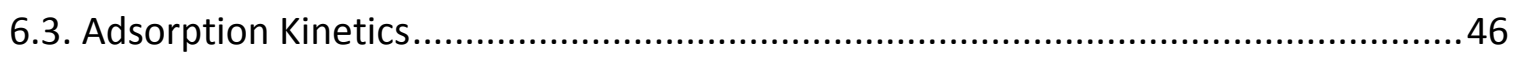

7. Characterization of Two-Component Adsorption Equilibrium and Kinetics of Antibody

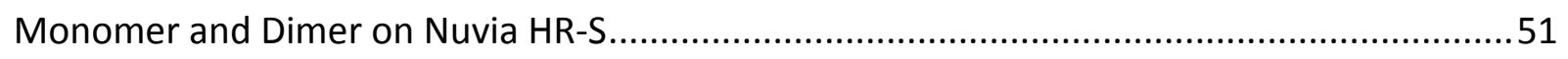

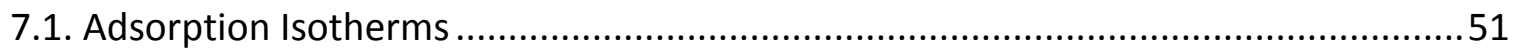

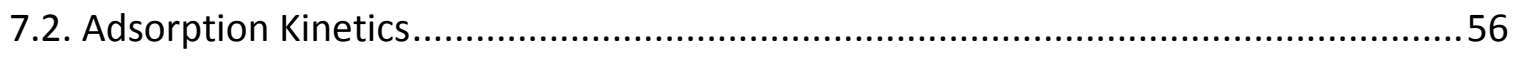

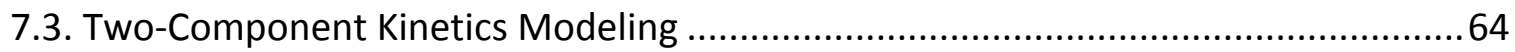

8. Frontal Analysis and Column Modeling of Monomer-Dimer Systems on Nuvia HR-S..............67

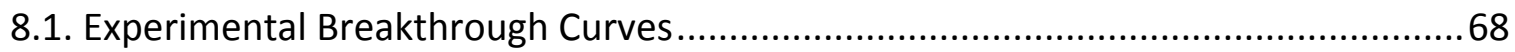

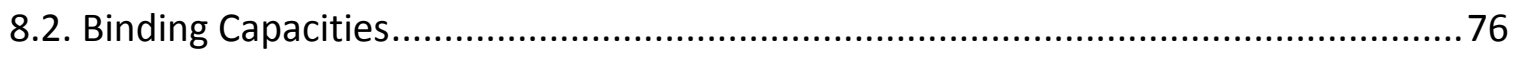

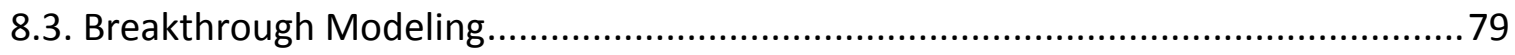

9. Kinetic Measurements via Biolayer Interferometry ………………………......................94

9.1. Probe functionalization attempts and characterization ...........................................97

9.2. Single Component Measurements ........................................................................... 101

9.3. Sequential Adsorption Measurements ..................................................................104

9.4. Modeling and Parameter Estimation ......................................................................107

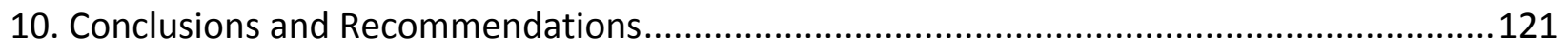

10.1. Single and Two-Component Adsorption Behavior ...............................................121

10.2. Frontal Analysis and Column Modeling ..............................................................124 


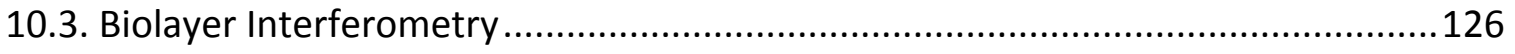

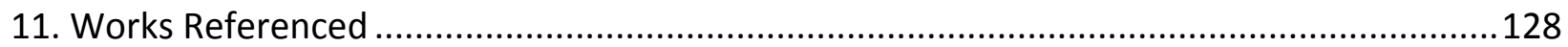

Appendix A: Numerical Model Derivation and Discretization .............................................140

Appendix B: Two-Component Adsorption Isotherms of Antibody Monomers ........................143 


\section{List of Figures}

Figure 1.1. Antibody approvals by year, and cumulative antibody approvals through October 2017. Bar denoting antibodies "Approved, Later Removed" were at one point approved by the FDA, but subsequently removed from the market for various reasons. Figure adapted from Ecker et al. (2015).

Figure 1.2. Traditional downstream "platform" purification process. Figure adapted from Shukla et al. (2007) .5

Figure 3.1. Sensorgram schematic for (a) single component protein binding experiments and (b) sequential protein binding experiments. $A$ is a baseline equilibration in load buffer, $B$ is the first protein binding to a clean surface, $C$ is the first protein dissociating from the surface, $D$ is a protein desorption in high salt, $\mathrm{E}$ is the second protein binding to a preloaded surface, and $\mathrm{F}$ is the second protein dissociating from the surface.

Figure 4.1. (a) Representative micrograph image and (b) particle size distribution for Nuvia HR-S taken from microphotographs at 10x magnification.

Figure 4.2. Sample TEM images of Nuvia HR-S at (a) 8000x and (b) 20000x magnification.........25

Figure 4.3. Inverse size exclusion chromatography peaks for glucose and dextran probes on Nuvia HR-S column

Figure 5.1. Analytical HPLC chromatograms of mAbs A through D on (a) C4 reverse phase column and (b) WCX-10 CEX column. MAb B chromatogram in (b) has been lightened to allow distinction from $\mathrm{mAb} C$

Figure 5.2. Analytical SEC chromatograms of (a) mAbs A-D and (b) mAb D dimer-rich sample. $r_{H}$-values for each species are reported in Table 5.1

Figure 5.3. IsoGel ${ }^{\circledR}$ Agarose pH 7-11 IEF Plate (Lonza Inc., Rockland, ME, USA) of mAb monomers A through D. Lane 1 contains Broad Range pl 4.45-9.6 IEF Standards (Bio-Rad Laboratories Inc., Hercules, CA, USA). Lane 2 through 5 contains mAb A through D monomers, respectively 34

Figure 5.4. IsoGel ${ }^{\circledR}$ Agarose pH 7-11 IEF Plate (Lonza Inc., Rockland, ME, USA) of mAb D monomer and dimer samples. Lane 1 contains Broad Range pl 4.45-9.6 IEF Standards (Bio-Rad 
Laboratories Inc., Hercules, CA, USA). Lane 2 contains mAb D monomer sample. Lane 3 contains mAb D dimer sample 35

Figure 6.1 Analytical SEC of (a) dimer-rich sample and (b) purified monomer and purified dimer samples. UV signal in (b) is normalized to yield the same peak area for both species .38

Figure 6.2. LGE chromatograms for (a) monomer and (b) purified dimer with gradients from 0 to $300 \mathrm{mM} \mathrm{NaCl}$ in 5, 10, 15, 25, and $40 \mathrm{CV}$. The dashed lines show the conductivity traces. The thick solid lines connect the conductivity values at which the peak elutes at each gradient slope

Figure 6.3. Log-log plot of normalized gradient slope, $\gamma$, vs. the $\mathrm{Na}^{+}$concentration at elution, $C_{\mathrm{Na}^{+}}^{E}$, for the LGE experiments of Fig. 6.2

Figure 6.4. Dimensionless van Deemter plot for monomer and dimer obtained from isocratic pulse elution under non-binding conditions. The reduced velocity, $v^{\prime}$, is multiplied times the ratio $\left[k^{\prime} /\left(1+k^{\prime}\right)\right]^{2}$ with $k^{\prime}=k_{\infty}^{\prime}$ to account for slight differences in retention

Figure 6.5. Single component adsorption isotherms for mAb monomer (filled symbols) and mAb dimer (open symbols). Solid and dashed lines represent the Langmuir model isotherm in (a) and the SMA model in (b) calculated using the parameters in Table 6.1...... 45

Figure 6.6. Representative CLSM images of single component adsorption of mAb monomer (left, red) and mAb dimer (right, green) on similarly sized resin particles at comparable times from $2.0 \mathrm{mg} / \mathrm{mL}$ solutions in $10 \mathrm{mM} \mathrm{Na}_{2} \mathrm{HPO}_{4}$ at $\mathrm{pH}$ 7.0. Actual times and particle diameters are shown in the insets.

Figure 6.7. Dimensionless front positions vs. reduced time for single component adsorption of mAb monomer and dimer for the conditions of Fig. 6.6. Lines are based on Eq. 6.7 with $D_{e} / D_{0}=$ 0.204 and 0.137 for the monomer and dimer, respectively. .48

Figure 6.8. LGE chromatograms of labeled and unlabeled (a) monomer and (b) dimer feedstocks on Nuvia HR-S. $10 \mathrm{CV}$ gradient from $10 \mathrm{mM} \mathrm{Na}_{2} \mathrm{HPO}_{4}, \mathrm{pH} 7.0$ to $10 \mathrm{mM} \mathrm{Na}_{2} \mathrm{HPO}_{4}, 300 \mathrm{mM} \mathrm{NaCl}$, $\mathrm{pH} 7.0$ .50

Figure 7.1. SEC chromatograms of two-component isotherm feed and supernatant for a mAb monomer and dimer mixture at $20 \mathrm{mM} \mathrm{Na}^{+}$at varying initial ratios of monomer and dimer. 
Solid line is of the isotherm feed before contact with resin, dashed line is isotherm supernatant at equilibrium. Inset text shows final total protein concentration $(\mathrm{mg} / \mathrm{mL})$ and ratio of monomer to dimer mass concentration .53

Figure 7.2. SEC chromatograms of two-component isotherm feed and supernatant for a mAb monomer and dimer mixture at (a) $20 \mathrm{mM}$, (b) $30 \mathrm{mM}$, (c) $50 \mathrm{mM}$, and (d) $80 \mathrm{mM} \mathrm{Na}{ }^{+}$. Sold line is the isotherm feed before contact with resin, dashed line is isotherm supernatant at equilibrium. Inset text shows final total protein concentration $(\mathrm{mg} / \mathrm{mL})$ and ratio of monomer to dimer mass concentration

Figure 7.3. Representative CLSM images of two-component adsorption of a mixture containing $1.15 \mathrm{mg} / \mathrm{mL} \mathrm{mAb}$ monomer and $1.02 \mathrm{mg} / \mathrm{mL} \mathrm{mAb}$ dimer in $10 \mathrm{mM} \mathrm{Na}{ }_{2} \mathrm{HPO}_{4}$ at $\mathrm{pH}$ 7.0. The top row (red) shows the monomer profile, the middle row (green) the dimer profile, and the bottom row a digital superposition of the monomer and dimer signals. Actual times and particle diameters are shown in the insets .58

Figure 7.4. Dimensionless front position vs. reduced time for pseudo one-component adsorption of mAb monomer and dimer for the conditions of Fig. 7.3. Line is based on Eq. 6.7 with averaged values of $q_{m}$ and $C$ for monomer and dimer

Figure 7.5. Representative CLSM images of adsorption of $1.91 \mathrm{mg} / \mathrm{mL} \mathrm{mAb}$ dimer on particles initially saturated with approximately $2 \mathrm{mg} / \mathrm{mL} \mathrm{mAb}$ monomer in $10 \mathrm{mM} \mathrm{Na} \mathrm{HPO}_{4}$ at pH 7.0. The top row (red) shows the monomer profile, the middle row (green) the dimer profile, and the bottom row a digital superposition of the monomer and dimer signals. Actual times and particle diameters are shown in the insets

Figure 7.6. Representative CLSM images of two-component adsorption of a mixture containing $1.15 \mathrm{mg} / \mathrm{mL} \mathrm{mAb}$ monomer and $0.96 \mathrm{mg} / \mathrm{mL} \mathrm{mAb}$ dimer in $10 \mathrm{mM} \mathrm{Na}{ }_{2} \mathrm{HPO}_{4}$ at pH 7.0 with 10 $\mathrm{mM}$ added $\mathrm{NaCl}$. The top row (red) shows the monomer profile, the middle row (green) the dimer profile, and the bottom row a digital superposition of the monomer and dimer signals. Actual times and particle diameters are shown in the insets

Figure 7.7. Representative CLSM images of two-component adsorption of a mixture containing $1.09 \mathrm{mg} / \mathrm{mL} \mathrm{mAb}$ monomer and $0.88 \mathrm{mg} / \mathrm{mL} \mathrm{mAb}$ dimer in $10 \mathrm{mM} \mathrm{Na}_{2} \mathrm{HPO}_{4}$ at pH 7.0 with 60 $\mathrm{mM}$ added $\mathrm{NaCl}$. The top row (red) shows the monomer profile, the middle row (green) the 
dimer profile, and the bottom row a digital superposition of the monomer and dimer signals. Actual times and particle diameters are shown in the insets

Figure 7.8. Representative CLSM images of two-component adsorption of a mixture containing $1.82 \mathrm{mg} / \mathrm{mL} \mathrm{mAb}$ monomer and $0.36 \mathrm{mg} / \mathrm{mL} \mathrm{mAb}$ dimer in $10 \mathrm{mM} \mathrm{Na} 2 \mathrm{HPO}_{4}$ at pH 7.0 with 60 $\mathrm{mM}$ added $\mathrm{NaCl}$. The top row (red) shows the monomer profile, the middle row (green) the dimer profile, and the bottom row a digital superposition of the monomer and dimer signals. Actual times and particle diameters are shown in the insets .63

Figure 7.9. Dimensionless front position vs. reduced time for two-component adsorption in 10 $\mathrm{mM} \mathrm{Na}_{2} \mathrm{HPO}_{4}$ at pH 7.0 with $60 \mathrm{mM}$ added $\mathrm{NaCl}$ for the conditions of (a) Fig. 7.7 and (b) Fig. 7.8 with $C_{M}$ and $C_{D}$ the same as described in those Figs. Lines are based on Eqs. 7.4 and 7.5 using $q_{m}$ and $D_{e}$ values obtained from single component measurements and $q^{*}$ values estimated from the two-component equilibrium measurements (Table 7.1) 66

Figure 8.1. Single component breakthrough curves of (a) monomer and (b) dimer in pH 7 load buffers containing 20 and $80 \mathrm{mM} \mathrm{Na}^{+}$. Feed concentrations were $2.0 \pm 0.1 \mathrm{mg} / \mathrm{mL}, u$ was 76 $\mathrm{cm} / \mathrm{h}$, column length was $4.4 \mathrm{~cm}$, and residence time was $3.5 \mathrm{~min}$. Dashed lines are model predictions neglecting any kinetic resistance to binding. Solid lines are fitted including a kinetic resistance to binding with rate constants given in Table 8.2 70

Figure 8.2. Binary breakthrough behavior of monomer-dimer mixture containing approximately $30 \%$ dimer in $\mathrm{pH} 7$ load buffer containing $20 \mathrm{mM} \mathrm{Na}^{+}$(a). SEC analysis of the experiment feed mixture (solid line) and the protein pool eluted in $1 \mathrm{M} \mathrm{NaCl}$ (dashed line) (b). $u$ was $150 \mathrm{~cm} / \mathrm{h}$, column length was $10.6 \mathrm{~cm}$, and residence time was $4.2 \mathrm{~min}$. Exact feed composition given in Table 8.1 .71

Figure 8.3. Binary breakthrough behavior of monomer-dimer mixture containing approximately $30 \%$ dimer in $\mathrm{pH} 7$ load buffer containing $30 \mathrm{mM} \mathrm{Na}^{+}$(a). SEC analysis of the experiment feed mixture (solid line) and the protein pool eluted in $1 \mathrm{M} \mathrm{NaCl}$ (dashed line) (b). $u$ was $76 \mathrm{~cm} / \mathrm{h}$, column length was $5.9 \mathrm{~cm}$, and residence time was $4.6 \mathrm{~min}$. Exact feed composition given in Table 8.1

Figure 8.4. Binary breakthrough behavior of monomer-dimer mixture containing approximately $30 \%$ dimer in $\mathrm{pH} 7$ load buffer containing $50 \mathrm{mM} \mathrm{Na}^{+}$(a). SEC analysis of the experiment feed 
mixture (solid line) and the protein pool eluted in $1 \mathrm{M} \mathrm{NaCl}$ (dashed line) (b). $u$ was $76 \mathrm{~cm} / \mathrm{h}$, column length was $5.9 \mathrm{~cm}$, and residence time was $4.6 \mathrm{~min}$. Exact feed composition given in Table 8.1

Figure 8.5. Binary breakthrough behavior of monomer-dimer mixture containing approximately $30 \%$ dimer in $\mathrm{pH} 7$ load buffer containing $80 \mathrm{mM} \mathrm{Na}^{+}$(a). SEC analysis of the experiment feed mixture (solid line) and the protein pool eluted in $1 \mathrm{M} \mathrm{NaCl}$ (dashed line) (b). $u$ was $76 \mathrm{~cm} / \mathrm{h}$, column length was $5.0 \mathrm{~cm}$, and residence time was $3.9 \mathrm{~min}$. Exact feed composition given in Table 8.1

Figure 8.6. Binary breakthrough behavior of monomer-dimer mixture containing approximately $30 \%$ dimer in $\mathrm{pH} 7$ load buffer containing $120 \mathrm{mM} \mathrm{Na}^{+}$(a). SEC analysis of the experiment feed mixture (solid line) and the protein pool eluted in $1 \mathrm{M} \mathrm{NaCl}$ (dashed line) (b). $u$ was $76 \mathrm{~cm} / \mathrm{h}$, column length was $5.6 \mathrm{~cm}$, and residence time was $4.4 \mathrm{~min}$. Exact feed composition given in Table 8.1 75

Figure 8.7. Comparison of binary breakthrough curves at $20 \mathrm{mM} \mathrm{Na}^{+}$with predictions based on Eqs. 8.6-8.8 using the SMA model (Eq. 8.5) to describe competitive binding of monomer and dimer. Experimental conditions are the same as in Fig. 8.2. Dashed lines show predictions without including a kinetic resistance to binding. Solid lines include a kinetic resistance to binding according to Eq. 8.8 with rate constants given in Table 8.2

Figure 8.8. Comparison of binary breakthrough curves at $30 \mathrm{mM} \mathrm{Na}^{+}$with predictions based on Eqs. 8.6 - 8.8 using the SMA model (Eq. 8.5) to describe competitive binding of monomer and dimer. Experimental conditions are the same as in Fig. 8.3. Dashed lines show predictions without including a kinetic resistance to binding. Solid lines include a kinetic resistance to binding according to Eq. 8.8 with rate constants given in Table 8.2

Figure 8.9. Comparison of binary breakthrough curves at $50 \mathrm{mM} \mathrm{Na}^{+}$with predictions based on Eqs. 8.6-8.8 using the SMA model (Eq. 8.5) to describe competitive binding of monomer and dimer. Experimental conditions are the same as in Fig. 8.4. Dashed lines show predictions without including a kinetic resistance to binding. Solid lines include a kinetic resistance to binding according to Eq. 8.8 with rate constants given in Table 8.2 
Figure 8.10. Comparison of binary breakthrough curves at $80 \mathrm{mM} \mathrm{Na}^{+}$with predictions based on Eqs. 8.6 - 8.8 using the SMA model (Eq. 8.5) to describe competitive binding of monomer and dimer. Experimental conditions are the same as in Fig. 8.5. Dashed lines show predictions without including a kinetic resistance to binding. Solid lines include a kinetic resistance to binding according to Eq. 8.8 with rate constants given in Table 8.2

Figure 8.11. Comparison of binary breakthrough curves at $120 \mathrm{mM} \mathrm{Na}^{+}$with predictions based on Eqs. 8.6 - 8.8 using the SMA model (Eq. 8.5) to describe competitive binding of monomer and dimer. Experimental conditions are the same as in Fig. 8.6. Dashed lines show predictions without including a kinetic resistance to binding. Solid lines include a kinetic resistance to binding according to Eq. 8.8 with rate constants given in Table 8.2

Figure 8.12. Binary breakthrough behavior of a $2 \mathrm{mg} / \mathrm{mL}$ monomer-dimer mixture containing approximately $15 \%$ dimer in $\mathrm{pH} 7$ load buffer containing $80 \mathrm{mM} \mathrm{Na}^{+}$(a). SEC analysis of the experiment feed mixture (solid line) and the protein pool eluted in $1 \mathrm{M} \mathrm{NaCl}$ (dashed line) (b). $u$ was $76 \mathrm{~cm} / \mathrm{h}$, column length was $3.0 \mathrm{~cm}$, and residence time was $2.4 \mathrm{~min}$. Lines in (a) are predictions based on Eqs. 8.6 - 8.8 including a kinetic resistance to binding with rate constants in Table 8.2

Figure 8.13. Comparison of experimental intraparticle concentration profiles of Fig. 7.3 (in 20 $\mathrm{mM} \mathrm{Na}^{+}$) with model predictions based on Eqs. 8.7 - 8.8. Solid and open circles are normalized fluorescence intensity profiles of the monomer and dimer, respectively. Lines are model predictions based on original experimental conditions

Figure 8.14. Comparison of experimental intraparticle concentration profiles of Fig. 7.7 (in 80 $\mathrm{mM} \mathrm{Na}^{+}$) with model predictions based on Eqs. 8.7 - 8.8. Solid and open circles are normalized fluorescence intensity profiles of the monomer and dimer, respectively. Lines are model predictions based on original experimental conditions

Figure 8.15. Comparison of experimental intraparticle concentration profiles of Fig. 7.8 (in 80 $\mathrm{mM} \mathrm{Na}^{+}$) with model predictions based on Eqs. 8.7 - 8.8. Solid and open circles are normalized fluorescence intensity profiles of the monomer and dimer, respectively. Lines are model predictions based on original experimental conditions .93 
Figure 9.1. Schematic representation of a biolayer interferometry probe with (a) no species bound and (b) after binding. Intensity plot based on Eq. 9.1 before (solid) and after (dashed) binding an example species (c). 96

Figure 9.2. Sensorgrams of BSA binding on AR2 $\mathrm{G}$ probes in $20 \mathrm{mM} \mathrm{NaCH}{ }_{3} \mathrm{COO}$ at pH 5.0 (solid) and in $10 \mathrm{mM} \mathrm{Na}_{2} \mathrm{HPO}_{4}$ at $\mathrm{pH} 7.0$ (dotted and inset). Changes between phases are marked in dashed vertical lines.

Figure 9.3. AR2G biosensor tip image at $4 \mathrm{x}$ magnification under (a) visible light and (b) fluorescence with bound mAb monomer 100

Figure 9.4. Sensorgrams for $\mathrm{mAb}$ monomer binding in $10 \mathrm{mM} \mathrm{Na}_{2} \mathrm{HPO}_{4}$ at $\mathrm{pH} 7.0$ with (a) $0 \mathrm{mM}$, (b) $30 \mathrm{mM}$, (c) $60 \mathrm{mM}$, and (d) $100 \mathrm{mM} \mathrm{NaCl}$. Reference sensor is shown as dotted line. Changes between phases are marked by dashed vertical lines .102

Figure 9.5. Sensorgrams for $\mathrm{mAb}$ dimer binding in $10 \mathrm{mM} \mathrm{Na}{ }_{2} \mathrm{HPO}_{4}$ at $\mathrm{pH} 7.0$ with (a) $0 \mathrm{mM}$, (b) $30 \mathrm{mM}$, (c) $60 \mathrm{mM}$, and (d) $100 \mathrm{mM} \mathrm{NaCl}$. Reference sensor is shown as dotted line. Changes between phases are marked by dashed vertical lines

Figure 9.6. Sensorgrams for sequential binding of $32 \mathrm{nM}$ mAb monomer followed by varying concentrations of mAb dimer in $10 \mathrm{mM} \mathrm{Na}_{2} \mathrm{HPO}_{4}$ at pH 7.0 with (a) $0 \mathrm{mM}$ and (b) $60 \mathrm{mM} \mathrm{NaCl}$. Reference sensors are shown as dotted lines. Changes between phases are marked by dashed vertical lines. 105

Figure 9.7. Sensorgrams for sequential binding of $17 \mathrm{nM}$ mAb dimer followed by varying concentrations of mAb monomer in $10 \mathrm{mM} \mathrm{Na}_{2} \mathrm{HPO}_{4}$ at $\mathrm{pH} 7.0$ with (a) $0 \mathrm{mM}$ and (b) $60 \mathrm{mM}$ $\mathrm{NaCl}$. Reference sensors are shown as dotted lines. Changes between phases are marked by dashed vertical lines 106

Figure 9.8. Sensorgrams for sequential binding of $34 \mathrm{nM} \mathrm{mAb}$ monomer in $10 \mathrm{mM} \mathrm{Na}_{2} \mathrm{HPO}_{4}$ at pH 7.0 with $0,10,30$, and $60 \mathrm{mM} \mathrm{NaCl}$ followed by (a) $34 \mathrm{nM}$ mAb monomer or (b) $17 \mathrm{nM} \mathrm{mAb}$ dimer in $10 \mathrm{mM} \mathrm{Na}_{2} \mathrm{HPO}_{4}$ at $\mathrm{pH} 7.0$ with $0 \mathrm{mM} \mathrm{NaCl}$. Changes between phases are marked by dashed vertical lines .108

Figure 9.9. Experimental data (open squares) and model fits for a one-site (solid line) and twosite (dashed line) binding model for antibody monomer in different concentrations of total $\mathrm{Na}^{+}$. 
Experimental conditions are the same as in Fig. 9.4. One-site model based on Eq. 9.2; two-site model based on Eq. 9.3 with parameters for both Eqs. given in Table 9.1

Figure 9.10. Experimental data (open squares) and model fits for a one-site (solid line) and twosite (dashed line) binding model for antibody dimer in different concentrations of total $\mathrm{Na}^{+}$. Experimental conditions are the same as in Fig. 9.5. One-site model based on Eq. 9.2; two-site model based on Eq. 9.3 with parameters for both Eqs. given in Table 9.1

Figure 9.11. Binding rate during association phase of monomer experiments depicted in Fig. 9.4 vs. total probe binding. Experimental conditions are the same as described in Fig. 9.4 116 Figure 9.12. Binding rate during association phase of dimer experiments depicted in Fig. 9.5 vs. total probe binding. Experimental conditions are the same as described in Fig. 9.5.....

Figure 9.13. Binding rate during second association phase of sequential adsorption experiments depicted in Fig. 9.8 vs. total probe binding. Experimental conditions are the same as described in Fig. 9.8 118

Figure 9.14. Experimental data (open squares) and model fits for a two-site binding model for sequential adsorption of $34 \mathrm{nM}$ antibody monomer in (a) $20 \mathrm{mM} \mathrm{Na}^{+}$and (b) $80 \mathrm{mM} \mathrm{Na}^{+}$ followed by $17 \mathrm{nM}$ antibody dimer in $20 \mathrm{mM} \mathrm{Na}^{+}$. Solid line is combined monomer and dimer model signal; dashed line is monomer model signal; dotted line is dimer model signal. Model lines based on numerical integration of Eq. 9.4 with parameters given in Table 9.1 120 Figure B.1. Protein retention factor due to binding vs. $\mathrm{Na}^{+}$concentration at retention for mAbs A - D from linear gradient elution experiments. .144

Figure B.2. Equilibrium isotherm measurements and calculated Langmuir isotherm fits for mAbs A, B, and D on Nuvia HR-S at $20 \mathrm{mM} \mathrm{Na}^{+}$(closed symbols, solid lines) and $80 \mathrm{mM} \mathrm{Na}^{+}$(open symbols, dashed lines) at $\mathrm{pH} 7.0$ 145

Figure B.3. Two-component adsorption equilibrium data for $\mathrm{mAb} B$ (filled circles) and $\mathrm{mAb} D$ (open circles) mixtures in $10 \mathrm{mM} \mathrm{Na}_{2} \mathrm{HPO}_{4}$ at pH 7.0 with (a) $0 \mathrm{mM}$ and (b) $60 \mathrm{mM} \mathrm{NaCl}$ added

Figure B.4. Two-component adsorption equilibrium data for mAb A (filled circles) and $\mathrm{mAb} D$ (open circles) mixtures in $10 \mathrm{mM} \mathrm{Na}_{2} \mathrm{HPO}_{4}$ at pH 7.0 with (a) $0 \mathrm{mM}$ and (b) $60 \mathrm{mM} \mathrm{NaCl}$ added 


\section{List of Tables}

Table 4.1. Inverse size exclusion chromatography probe molecular weights, correlated

hydrodynamic radii by Hagel et al. (1996), and measured distribution coefficients...... .27

Table 5.1. Protein properties and characterization data for mAbs A-D and mAb D dimer..... 36

Table 6.1. Langmuir and SMA isotherm parameters obtained by fitting the single-component adsorption data for monomer and dimer in $10 \mathrm{mM} \mathrm{Na}_{2} \mathrm{HPO}_{4}$ at $\mathrm{pH} 7.0$ with the addition of different $\mathrm{NaCl}$ concentrations

Table 7.1. Two-component adsorption equilibrium data for $\mathrm{mAb}$ monomer/dimer mixtures in $10 \mathrm{mM} \mathrm{Na}_{2} \mathrm{HPO}_{4}$ at $\mathrm{pH} 7.0$ with the addition of different $\mathrm{NaCl}$ concentrations .52

Table 8.1. Equilibrium binding capacities obtained from the single and binary breakthrough experiments compared with predictions from the SMA model..... .78

Table 8.2. Parameters used in numerical model calculations of Eqs. 8.6 - 8.8. Individual protein parameters from experiments in Chapter 6

Table 9.1. Kinetic parameters from one-site and two-site binding models for monomer and dimer at varying salt concentrations

Table B.1. Two-component adsorption equilibrium data for $\mathrm{mAb} B$ and $\mathrm{mAb} D$ mixtures in 10 $\mathrm{mM} \mathrm{Na}_{2} \mathrm{HPO}_{4}$ at $\mathrm{pH} 7.0$.

Table B.2. Two-component adsorption equilibrium data for $\mathrm{mAb} B$ and $\mathrm{mAb} D$ mixtures in 10 $\mathrm{mM} \mathrm{Na}_{2} \mathrm{HPO}_{4}$ at $\mathrm{pH} 7.0$ with the addition of $60 \mathrm{mM} \mathrm{NaCl}$ .149

Table B.3. Two-component adsorption equilibrium data for $\mathrm{mAb} A$ and $\mathrm{mAb} D$ mixtures in 10 $\mathrm{mM} \mathrm{Na}_{2} \mathrm{HPO}_{4}$ at $\mathrm{pH} 7.0$ with and without the addition of $60 \mathrm{mM} \mathrm{NaCl}$ 


\section{List of Symbols}

\section{Symbols}

A steric mass action constant during linear gradient elution experiments

$B \quad$ long-time value of autocorrelation function

Bi Biot number

$C \quad$ protein concentration in solution $(\mathrm{mg} / \mathrm{mL})$

$C_{0} \quad$ initial protein concentration in solution in isotherm measurements $(\mathrm{mg} / \mathrm{mL})$

$C_{F} \quad$ protein feed concentration in frontal analysis experiments $(\mathrm{mg} / \mathrm{mL})$

$\mathrm{C}_{\mathrm{Na}^{+}}$sodium concentration in solution (mM)

$C_{\mathrm{Na}^{+}}^{E} \quad$ sodium concentration at elution during linear gradient elution experiments (mM)

$c \quad$ protein concentration in resin pores $(\mathrm{mg} / \mathrm{mL})$

$C V_{s h}$ emergence of shock front during frontal analysis experiments in column volumes

$D_{0} \quad$ free solution diffusivity $\left(\mathrm{cm}^{2} / \mathrm{s}\right)$

$D_{e} \quad$ effective diffusivity $\left(\mathrm{cm}^{2} / \mathrm{s}\right)$

$d \quad$ distance between reflecting surfaces in BLI experiments $(\mathrm{nm})$

$d_{p} \quad$ particle diameter $(\mu \mathrm{m})$

$\bar{d}_{p} \quad$ volume-averaged particle diameter $(\mu \mathrm{m})$

$f \quad$ volume fraction of particle during particle sizing measurements

$g^{(2)} \quad$ autocorrelation function of the intensity of scattered light

$I \quad$ intensity of scattered light

$K_{D} \quad$ distribution coefficient

$K_{e} \quad$ equilibrium constant in steric mass action isotherm

$K_{L} \quad$ affinity constant in Langmuir isotherm $(\mathrm{mL} / \mathrm{mg})$

$k \quad$ kinetic rate constant in column modeling

$k_{B} \quad$ Boltzmann constant

$k_{a} \quad$ association rate constant in BLI modeling $\left(\mathrm{M}^{-1} \mathrm{~s}^{-1}\right)$

$k_{d} \quad$ dissociation rate constant in BLI modeling $\left(\mathrm{s}^{-1}\right)$ 
$k_{f} \quad$ film mass transfer coefficient $(\mathrm{cm} / \mathrm{s})$

$k^{\prime} \quad$ retention factor

$k_{\text {bind }}^{\prime} \quad$ protein retention factor due to binding

$k_{\infty}^{\prime} \quad$ protein non-binding retention factor

$L \quad$ column length $(\mathrm{cm})$

$M_{r} \quad$ protein molecular weight (Da)

$M_{w} \quad$ total mass of centrifuged resin in isotherm measurements (mg)

$n \quad$ refractive index of solution

$q \quad$ adsorbed phase protein concentration $(\mathrm{mg} / \mathrm{mL})$ or magnitude of scattering wave vector in dynamic light scattering measurements

$q_{0} \quad$ resin charge density in steric mass action isotherm (mM)

$q_{m} \quad$ monolayer binding capacity in Langmuir isotherm $(\mathrm{mg} / \mathrm{mL})$

$q^{*} \quad$ equilibrium adsorbed phase concentration $(\mathrm{mg} / \mathrm{mL})$

$\langle\bar{q}\rangle \quad$ total average protein concentration in resin particles (mg protein $/ \mathrm{mL}$ resin)

$R \quad$ binding signal in BLI experiments $(\mathrm{nm})$

$R_{\max } \quad$ maximum binding signal in $\mathrm{BLI}$ experiments $(\mathrm{nm})$

$r \quad$ particle radial coordinate in column modeling

$r_{H} \quad$ hydrodynamic radius $(\mathrm{nm})$

$r_{p} \quad$ particle radius $(\mu \mathrm{m})$

$r_{\text {pore }} \quad$ resin pore radius $(\mathrm{nm})$

$r_{s} \quad$ radial position of adsorption front in confocal microscopy experiments $(\mu \mathrm{m})$

T temperature

$t \quad$ time

$t_{0} \quad$ offset time at the start of dissociation phase in BLI experiments

$u \quad$ superficial velocity $(\mathrm{cm} / \mathrm{s})$

$V \quad$ total solution volume in isotherm measurements $(\mathrm{mL})$

$V_{C} \quad$ column volume $(\mathrm{mL})$ 
$V_{R} \quad$ adjusted retention volume $(\mathrm{mL})$

$v \quad$ interstitial velocity $(\mathrm{cm} / \mathrm{s})$

$v^{\prime} \quad$ reduced velocity

$x \quad$ column axial coordinate in column modeling

$z \quad$ protein effective charge

\section{Greek Symbols}

$\alpha_{i, j} \quad$ resin selectivity for species $i$ over species $j$

$\beta \quad$ gradient slope $(\mathrm{mM} / \mathrm{min})$ or system geometry factor for dynamic light scattering measurements

$\varepsilon \quad$ column extraparticle void fraction/porosity

$\varepsilon_{p} \quad$ resin intraparticle void fraction/porosity

$\eta \quad$ solution dynamic viscosity

$\Gamma \quad$ decay rate during dynamic light scattering measurements

$\gamma \quad$ normalized gradient slope (mM)

$\lambda_{0} \quad$ laser wavelength in a vacuum

$\rho_{r} \quad$ resin density $(\mathrm{g} / \mathrm{mL})$

$\rho_{s} \quad$ dimensionless front position in confocal microscopy experiments

$\sigma \quad$ steric hindrance parameter in steric mass action isotherm

$\tau \quad$ delay time during dynamic light scattering measurements

$\tau_{b} \quad$ estimated time scale for binding

$\tau_{D} \quad$ estimated time scale for diffusion

$\theta \quad$ scattering angle in dynamic light scattering measurements 


\section{Introduction and Background}

\subsection{Monoclonal Antibodies as a Therapeutic Protein}

Nearly 40 years ago, advances in molecular biology, cell culture techniques, and bioengineering allowed scientists at Genentech to express and purify recombinant insulin (Goeddel et al. 1979). The resulting FDA approval in 1982 (licensed to Eli Lilly as Humulin) marked the first small step in the creation of, previously unachievable, recombinant protein therapeutics (Leader et al. 2008; Carter 2011). Four years later in 1986, the FDA gave approval to JanssenCilag for Orthoclone OKT3 (Leavy 2010; Liu 2014), the first recombinant monoclonal antibody $(\mathrm{mAb})$, a class of proteins that has since grown to become the most successful of all biologic therapeutics. Figure 1.1 shows antibody approvals by year, as well as cumulative antibody approvals in the US through October 2017, adapted from Ecker et al. (2015) and updated with additional approvals since original publication (Jarvis 2015; Jarvis 2016; Jarvis 2017; Reichert 2017). With over 350 mAbs estimated to be in various phases of clinical development (Reichert 2013) and nearly $75 \%$ of all antibody approvals occurring in the past 10 years, monoclonal antibodies are projected to continue their growth for the foreseeable future. In 2015 , sales of biologics reached an estimated 184 billion dollars, accounting for $24 \%$ of global sales of all pharmaceuticals (Evaluate Ltd. 2016). And within the subset of biologics, mAbs accounted for nearly half of those sales, reaching an estimated 85 billion dollars in global sales (Grand View Research Inc. 2016).

Biologically, the advantages of mAbs as a therapeutic drug are numerous (Leader et al. 2008; Imai \& Takaoka 2006). In general, the complexity of proteins grants them a binding specificity and functional capability that cannot be replicated with relatively simple small molecule compounds. Because of native antibodies' role in the human immune system, mAbs are also uniquely suited to utilize these advantages to their fullest as a therapeutic. The vast library of antibodies in nature allow scientists to generate antibodies that can target virtually any receptor (Hayhurst \& Georgiou 2001; Liao et al. 2009). The large variety in mAbs can be partially attributed to their structure, which is comprised of a large, very highly conserved region and two much smaller variable regions that confer target specificity. The specificity of 


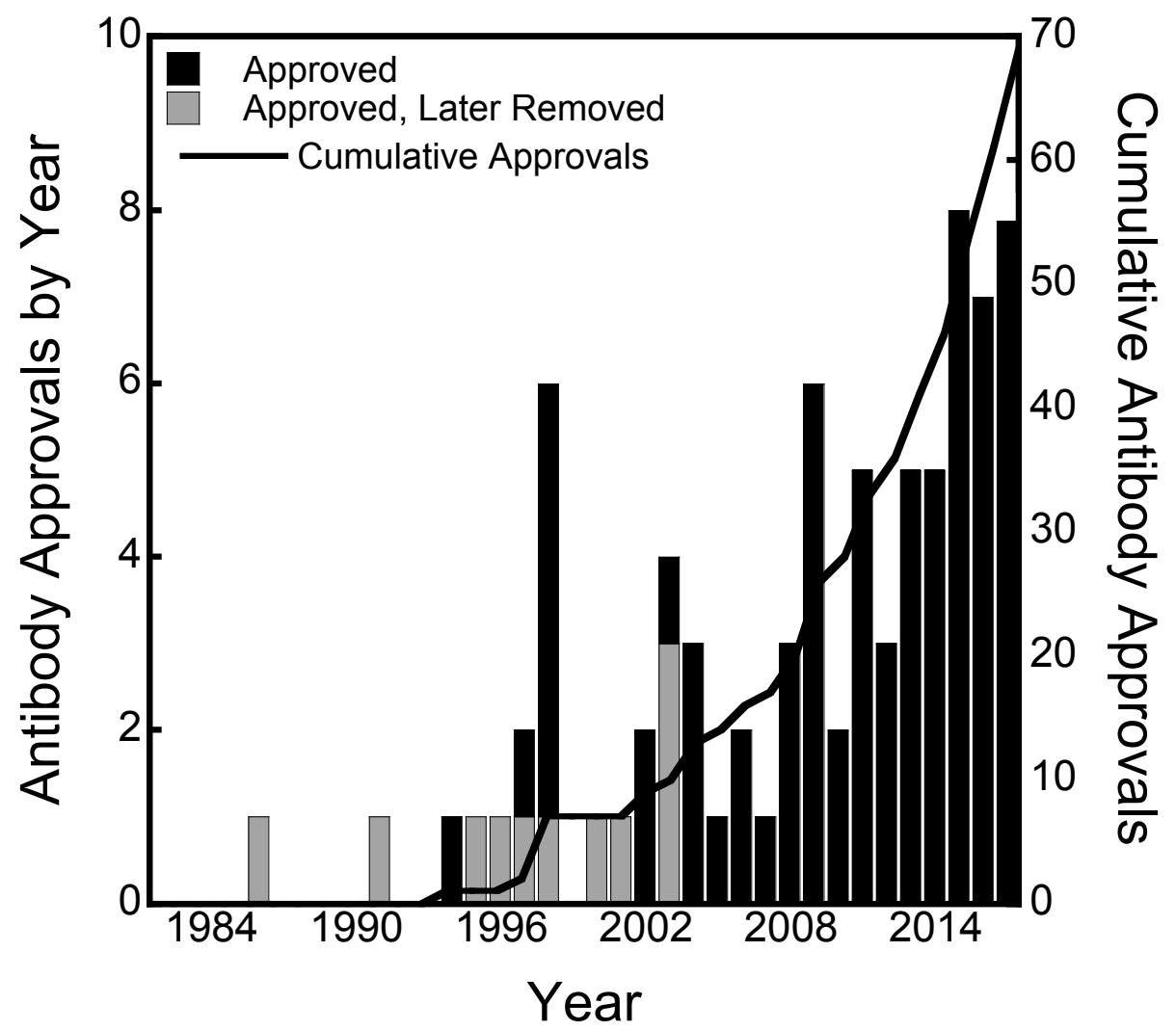

Figure 1.1. Antibody approvals by year, and cumulative antibody approvals through October 2017. Bar denoting antibodies "Approved, Later Removed" were at one point approved by the FDA, but subsequently removed from the market for various reasons. Figure adapted from Ecker et al. (2015) 
these variable regions tends to minimize potential side effects compared to small molecules, and also allows for a lower dosage in a patient. Additionally, the humanization of mAbs allows them to be well-tolerated by the body's existing immune system when administered as a drug (Lonberg 2005; Lonberg 2008). The suitability of mAbs as a therapeutic are borne out in their approval rates, which are typically higher than $20 \%$, compared to approximately $10 \%$ for small molecule drugs (Reichert 2003; Reichert et al. 2005).

However, the advantages of protein complexity do come at a price, as proteins are significantly more costly to produce and formulate than small molecules. Yet, mAbs are again uniquely suited to minimize these costs. The structural similarities of mAbs in their highly-conserved regions allow for very similar production approaches to mAbs of very different pharmaceutical application. These so called "platform" approaches have made both the upstream (Wurm 2004) and downstream (Shukla et al. 2007) purification development of mAbs significantly faster than that of other, non-mAb biopharmaceuticals. The downstream production of mAbs has become so reliant on this platform approach, that some claim that further work in downstream development could be better spent elsewhere (Kelley 2009), though others continue to develop and investigate new purification techniques (Low et al. 2007; Gagnon 2012).

\subsection{Downstream Processing of Monoclonal Antibodies}

The downstream processing of antibodies traditionally involves a series of chromatography and filtration steps (Shukla et al. 2007; Low et al. 2007; Kelley 2009; Hanke \& Ottens 2014) in order to meet impurity clearance standards and specifications set by the FDA and other health governing bodies (Food and Drug Administration 1999). These impurities are divided into two main groups: process-related impurities and product-related impurities. Process related impurities are further divided into three classifications: cell-substrate derived impurities (e.g. host cell proteins, host cell DNA), cell culture derived impurities (e.g. cell culture media components), and downstream-derived impurities (e.g. reagents, solvents, leachables). The latter two of these sub-groups can generally be mitigated through judicious processing choices 
while the former (host cell proteins in particular) often requires multiple orthogonal steps due to diversity in the characteristics of the impurities (Levy et al. 2014). Product-related impurities are molecular variants of the desired product (e.g. clipped species, aggregates, glycoforms) that often arise during the cell culture or fermentation steps (Dengl et al. 2013; Kunert \& Reinhart 2016), though they can also be generated during processing (Cromwell et al. 2006; Vázquez-Rey \& Lang 2011). In addition to the aforementioned impurities, processes for mammalian-derived products must also demonstrate the ability to clear viruses that may infect the cell culture (Food and Drug Administration 1998).

A traditional downstream process is shown in Fig. 1.2. Following the removal of cell debris via centrifugation and depth filtration, Protein A chromatography is used for the capture of the antibody product. Acting through an affinity mechanism that binds the conserved region of antibodies, Protein $\mathrm{A}$ is the workhorse of the mAb downstream process, often able to achieve purities in excess of 95\% from the clarified cell culture (Fahrner et al. 2001; Shukla \& Hinckley 2008). The antibody is eluted from Protein A at a low pH (Shukla et al. 2005), and then held at the low pH in order to inactivate viruses (Mattila et al. 2016). Following titration from the low $\mathrm{pH}$ conditions, polishing chromatography steps are used to remove the remaining impurities. Due to the high pl of most antibodies, anion exchange chromatography is often used and operated in a 'flowthrough' mode, binding acidic host cell proteins, negatively charged viruses, and host cell DNA while allowing the positively charged antibody product to pass through the column without interacting with the anion exchange media. The impurities removed via anion exchange chromatography represent the vast majority of process-related impurities, and thus, whether any additional polishing chromatography steps are necessary is typically product dependent. The remaining impurities are often product-related impurities, which can be difficult to characterize and separate. Cation exchange, mixed-mode, and hydrophobic interaction chromatography are all commonly used to remove these product-related impurities (Gagnon 2012). The typical downstream process is finalized with a nanofiltration step to remove potential virus contaminants, followed by an ultrafiltration/diafiltration step to concentrate the protein into its final formulation buffer. 


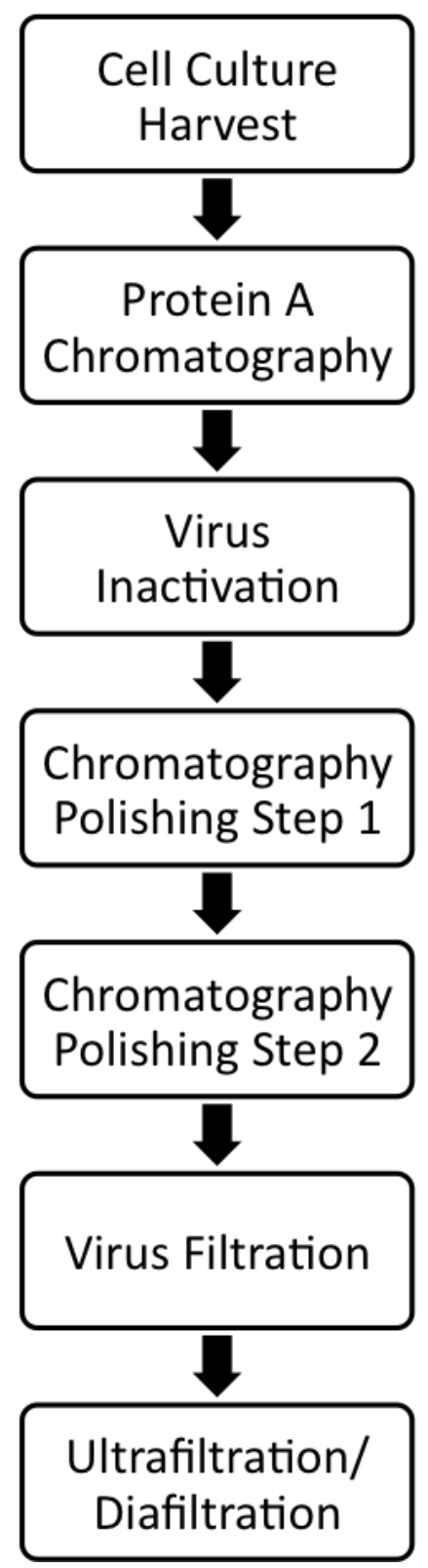

Figure 1.2. Traditional downstream "platform" purification process. Figure adapted from Shukla et al. (2007) 


\subsection{Aggregate Removal in Downstream Processing}

This platform approach has been an integral part in the successful commercialization of antibody-based therapies; however, advances in upstream technology as well as next generation therapeutic schemes will challenge the continued reliance on the traditional mAb platform (Carter 2011). The improvements in upstream processing, which have produced higher product titers, have also produced much more challenging impurity profiles. Of the fine impurities, protein aggregates are one of the most common and problematic impurities encountered in downstream processing of mAbs. A product-related impurity, they can be generated during cell cultivation (Dengl et al. 2013), formed during downstream processing when harsh conditions (e.g. low pH and/or high concentration of chaotropes) are used (Vázquez-Rey \& Lang 2011), or as a result of binding to certain chromatography surfaces (Guo et al. 2014). In addition to a potentially lower efficacy than their monomeric form (Vázquez-Rey \& Lang 2011), mAb aggregates are also more likely to generate an immunogenic response (Rosenberg 2006), and can result in less stable formulations (Wang et al. 2007), making them a key impurity attribute monitored during downstream processing. The removal of insoluble aggregates or higher-order soluble aggregate species can typically be achieved through filtration or with relatively simply chromatographic processes (Ansaldi \& Lester 2003), owing to the large difference in size and charge compared to the monomer. However, chromatographic separations of antibody dimers or other soluble species with modest differences in molecular mass from the monomeric form is often challenging, due in part to low selectivity in most chromatographic media, and in part to slow mass transfer. These difficult separations may require the use of operationally demanding techniques at manufacturing scale, such as gradient elution and/or small chromatographic bead sizes to achieve the necessary column efficiencies.

Currently, the separation of soluble antibody aggregates is most commonly achieved in manufacturing scale processes during the final polishing step using hydrophobic interaction chromatography (HIC) (Kramarczyk et al. 2008), mixed-mode chromatography (MMC) (Chmielowski et al. 2014), or cation exchange chromatography (CEX) (Kelley et al. 2008). Size 
exclusion chromatography (SEC) can be effective, but its generally low productivity and high plate number requirements make this technique unattractive. HIC based separations are reliant on the aggregates being more hydrophobic than the corresponding monomers, as they are frequently formed from unfolded intermediates. While also effective, this tool depends on complex protein-surface interactions and requires the use of high concentrations of kosmotropes that reduce solubility, and stationary phases that can lead to surface-induced conformational changes and unfolding, resulting in low recoveries (Jungbauer et al. 2005). MMC utilizes both hydrophobic and electrostatic interactions to, in theory, increase selectivity; however, this results in some of the same drawbacks as HIC while also requiring significant empirical data due to the complexity of the competing interactions (Toueille et al. 2011). CEX, on the other hand, is based on the higher charge of aggregates compared to the monomer, which generally results in stronger binding. Because of the generally milder operating conditions and more predictable behavior, CEX is often preferred.

\subsection{Cation Exchange Chromatography}

As stated above, CEX is often predictable, and the interaction of proteins with porous CEX resins in single component systems is relatively well understood with regards to both equilibrium (e.g. Kopaciewicz et al. 1983; Velayudhan \& Horvath 1988; Brooks \& Cramer 1992; DePhillips \& Lenhoff 2001; Roth et al. 1996) and kinetics (e.g. Ljunglöf \& Hjorth 1996; Ljunglöf \& Thömmes 1998; Hubbuch, Linden, Knieps, Thömmes, et al. 2003; Hubbuch, Linden, Knieps, Ljunglöf, et al. 2003; Hubbuch et al. 2002; Carta et al. 2005). Extending these well-developed equilibrium and kinetic models to describe the competitive behavior of multiple proteins binding simultaneously is of obvious practical interest.

A few studies have investigated the competitive binding of well-characterized model proteins. Weinbrenner and Etzel (1994), for example, showed the occurrence of concentration overshoots during the frontal loading of a strong cation exchange membrane with either mixtures of BSA and $\alpha$-lactalbumin or mixtures of BSA monomer and dimer. In both cases, the 
overshoot was attributed to competitive binding. Gadam et al. (1995) investigated the displacement-based separation of mixtures of $\alpha$-chymotrypsinogen and cytochrome $\mathrm{c}$ using a sulfated dextran displacer with a strong CEX resin. Martin et al. (2005) investigated the competitive binding kinetics of lysozyme and cytochrome $\mathrm{c}$ using finite bath and shallow bed methods. These studies demonstrated that the competitive binding of relatively small, but also very different, proteins occurred over time scales that are shorter than those associated with intraparticle diffusion.

Additional studies on larger molecules, such as mAbs, have corroborated many of the results from smaller proteins. Linden et al. (1999) used confocal laser scanning microscopy (CLSM) to visualize the simultaneous adsorption of polyclonal IgG and BSA on individual CEX resin beads. As shown by these authors, relatively sharp fronts were established within the resin, suggesting that the competitive binding of the two species occurred on time scales shorter than intraparticle diffusion. More recently, Tao et al. (2011, 2011, 2012) studied the competitive binding of a deamidated mAb mixture on both macroporous and polymer-grafted CEX resins in both batch and column experiments. Competitive binding of native and deamidated mAb species was shown to take place at a rate limited only by intraparticle diffusion for both resin types. However, while the rate was essentially independent on the direction of transport for the macroporous resin, the rates observed for the polymer-grafted resin were different dependent on whether native and deamidated isoforms were adsorbed simultaneously or in a consecutive manner, with the native species displacing the deamidated isoforms. Tao et al. (2011), Pérez-Almodóvar et al. (2012), and Zhu and Carta (2014) also used CLSM to study the evolution of intraparticle concentration profiles during adsorption of protein mixtures in CEX and $A E X$ resins for, respectively, demidated mAb mixtures, multiple mAbs, and mixtures of native and PEGylated proteins. These authors considered both macroporous and polymergrafted resins. For all three protein systems, simultaneous adsorption resulted in the formation of distinct bands within the resin bead in the case of the macroporous molecules, consistent with rapid displacement of the weaker binding species by the stronger binding one. Smooth profiles and slow rates for consecutive binding were seen however for adsorption in the 
polymer-grafted materials, indicating that transport and mechanisms are fundamentally different in these matrices. A detailed review addressing many of the underlying phenomena of protein adsorption and transport in polymer-grafted resins can be found in Lenhoff (2011).

Finally, several models have been developed to describe competitive protein binding equilibrium and kinetics for model systems in both macroporous materials and in polymergrafted resins (Martin et al. 2005; Tao et al. 2012; Lenhoff 2011; Xu \& Lenhoff 2009; Borg et al. 2014). These models and others have been used in industrial case studies to quantify the separation of mAb aggregates. Ansaldi and Lester (2003), for example, have described the use of salt steps and salt gradients to separate dimers and multimers from protein monomers on both weak and strong CEX resins. Zhou et al. (2007) demonstrated aggregate clearance from a $\mathrm{mAb}$ feedstock utilizing salt gradients and hybrid $\mathrm{pH}$-salt gradients with both weak and strong CEX resins. Suda et al. (2009) compared and modeled mAb aggregate clearance using both gradient elution and flow-through operation on macroporous and polymer-grafted CEX resins. Although useful as a design tool and to help define the critical parameter space, absent a more fundamental understanding of the underlying phenomena, such models have required parameterization through extensive experimental data. By probing and understanding the fundamentals of adsorption of monomers and their aggregates, it may be possible to reduce the development burden that these separations demand. 


\section{Research Scope and Objectives}

The primary purpose of this dissertation is to fundamentally understand the two-component behavior of a monoclonal antibody $(\mathrm{mAb})$ monomer and its soluble dimer on a cation exchange resin. This will involve a multi-scale approach that will attempt to use surface level, particle level, and column level experiments to put forth a coherent description of the two-component monomer-dimer behavior.

A full description of the two-component behavior must begin with an understanding of the single component behavior. This involves determination of the adsorption equilibrium and kinetics of the monomer and dimer on a macroporous CEX resin, with the goal of understanding how the increased size and charge of the dimer affect the interactions with the stationary phase. The equilibrium behavior will be determined at both high and low protein loadings using batch adsorption isotherms and linear gradient elution chromatography, respectively. These techniques will be used to extract isotherm parameters, which can be used with available adsorption models to describe the system. The kinetics behavior will be determined at both high and low protein loadings using confocal laser scanning microscopy and isocratic elution experiments under non-binding conditions, respectively. These techniques will be used to determine the mass transfer mechanism and rate of adsorption.

With an understanding of the single component behavior, the two-component behavior can be experimentally examined. The equilibrium behavior will be determined using two-component adsorption isotherms and the kinetics behavior will be determined using simultaneous and sequential adsorption with confocal microscopy. The equilibrium and kinetics responses will be compared to available model predictions based on the single component data in order to evaluate any complexities that may arise due to moving to a two-component system. Following these single-particle level measurements and modeling, the chromatographic response of the monomer-dimer system will be evaluated and modeled on the column scale. This is ultimately a more practical setting to evaluate and model the information learned from the single-particle level measurements done previously. 
Finally, the actual ion-exchange mechanism for the monomer and dimer will be examined using biolayer interferometry. This technique allows for a surface level evaluation of the association and dissociation kinetics of the protein adsorbing and desorbing to an ion-exchange surface. These kinetic measurements can be done for the single component case of a protein adsorbing to a clean surface, as well as a sequential case where a protein encounters an already loaded surface, giving insight into what effect, if any, the exchange of these macromolecules has with respect to the overall adsorption kinetics. Additionally, these experiments have the advantage of decoupling intraparticle mechanisms of transport, such as diffusion. While likely to be only qualitative, these measurements will hopefully give some insight into observed complexities in the monomer and dimer two-component behavior on the chromatographic media.

In summary, this dissertation will seek to meet the following goals:

1. Determine the physical characteristics of the chromatographic resin and the antibody monomer and dimer used in the work.

2. Characterize the single component adsorption equilibrium and kinetics of the monomer and dimer on the resin at the particle level. These results will be evaluated over a range of protein and salt concentrations and compared to available models.

3. Characterize the two-component adsorption equilibrium and kinetics of the monomer and dimer on the resin at the particle level. These results will be evaluated over a range of protein and salt concentrations and compared to available models.

4. Evaluate the one and two-component column behavior of the monomer and dimer system as a function of salt and protein concentration. These results will be numerically modeled using the particle level understanding of the one and twocomponent adsorption equilibrium and kinetics to capture the observed experimental behavior.

5. Determine the surface level exchange behavior of the monomer and dimer as a function of salt using biolayer interferometry. These results will be compared to the observed experimental behavior of the monomer and dimer system on the column and at the particle level. 
The first goal, the characterization of the chromatographic resin and antibody proteins, is addressed in Chapters 4 and 5, respectively. Chapter 6 details the second goal through adsorption isotherms, linear gradient elution, non-binding pulse injections, and confocal scanning laser microscopy. Chapter 7 uses similar techniques, but in a two-component system to address the third goal. The fourth goal is examined in Chapter 8 through experimental breakthrough curves of the monomer and dimer on a packed column. This chapter also details the development of a numerical model to accurately capture the observed experimental behavior. Finally, Chapter 9 utilizes biolayer interferometry to attempt to quantitate the exchange kinetics of the monomer and dimer, in fulfillment of the fifth and final goal. While these experiments do not represent a direct comparison to the internal pore structure of the chromatographic resin, they attempt to qualitatively corroborate the observed experimental results of the earlier chapters. 


\section{Materials \& Experimental Methods}

\subsection{Materials}

\subsubsection{Stationary Phase}

The primary stationary phase used throughout this work is Nuvia HR-S from Bio-Rad Laboratories, Inc. (Hercules, CA, USA). The resin possesses a macroporous backbone based on acrylamido and vinyl co-polymers functionalized with sulfonic acid groups. Its structure is similar to that of UNOsphere S and Rapid S, which have apparent pore sizes around $120 \mathrm{~nm}$ based on inverse size exclusion chromatography (Hunter \& Carta 2000). The manufacturer reported nominal particle diameter is $50 \pm 10 \mu \mathrm{m}$. The resin was chosen because of its inertness toward catalyzing the on-column formation of aggregates compared to other resins (Guo \& Carta 2015), relatively large pore size to allow diffusion with comparatively low hindrance, and small particle size to provide relatively fast kinetics.

\subsubsection{Proteins and Chemicals}

All antibodies used in this work were provided by Medlmmune (Gaithersburg, MD, USA) in purified bulk solutions of approximately $30 \mathrm{~g} / \mathrm{L}$ in histidine buffer at pH 6.5 or phosphate buffer at $\mathrm{pH}$ 7.0. This includes 4 separate high-purity IgG1 monoclonal antibodies ( $M_{r} \approx 150,000 \mathrm{Da}$ ) containing low levels of higher molecular weight species, and a solution of an antibody monomer enriched primarily with soluble antibody aggregates, assumed to be primarily dimers $\left(M_{r} \approx 300,000 \mathrm{Da}\right)$. This latter sample was obtained as the aggregate-rich fraction from a hydroxyapatite chromatography step following low-pH elution from a Protein A column. All buffering species and salts were purchased from Thermo Fisher Scientific (Waltham, MA, USA) and MilliporeSigma (St. Louis, MO, USA). Rhodamine Red-X and Green-X succinimidyl ester amine-reactive dyes were purchased from Life Technologies (Waltham, MA, USA). IsoGel Agarose IEF plates pH 3-10 were purchased from Lonza Rockland (Rockland, ME, USA). Isoelectric focusing gel pH 4.5 - 9.6 standards and staining solution were purchased from BioRad Laboratories (Hercules, CA, USA). All additional chemicals were purchased from Thermo Fischer Scientific and MilliporeSigma unless otherwise noted. 


\subsection{Experimental Methods}

\subsubsection{Particle Size Distribution}

The particle size distribution of the resin was calculated based on a volume-average of individual hydrated resin beads viewed on a Nikon Eclipse E200 microscope with Nikon E-Plan Achromat 10x, NA 0.25 WD $7 \mathrm{~mm}$ objective. A pixel to length ratio was established by imaging a reference standard with a known length. Images were converted to a black and white binary image in ImageJ, and individual beads were selected by using a circularity filter of $0.85-1.0$. The area of each particle was then calculated, and converted into a diameter and volume fraction.

\subsubsection{Particle Density}

The density of resin used in the work was measured in a $10 \mathrm{~mL}$ pycnometer. The total pycnometer volume was calculated to be $8.98 \mathrm{~mL}$ by a mass balance of the dry, empty pycnometer and the pycnometer filled with water at a known temperature and, thus, density. A slurry of resin equilibrated in water was then added to Corning ${ }^{\circledR}$ Spin- $X^{\circledR}$ microfiltration tubes and spun at 5000 RPM for 15 minutes in an Eppendorf Minispin ${ }^{\circledR}$ bench-top centrifuge to remove interstitial liquid. The resulting dry mass of resin was added to the pycnometer, and then the remaining volume filled with water at a known temperature. A resin particle density was then ascribed to the dry resin mass in order to complete the material balance of the resin and water slurry compared to the measured pycnometer volume. The resin particle density for Nuvia HR-S was calculated to be $1.08 \mathrm{~g} / \mathrm{mL}$.

\subsubsection{Isoelectric Focusing}

The isoelectric point of the various proteins was measured using precast IsoGel Agarose IEF 3$10 \mathrm{pH}$ plates purchased from Lonza Rockland (Rockland, ME, USA) using a GE Mulitphor II electrophoresis system from GE Healthcare Bio-Sciences AB (Uppsala, Sweden) cooled to $10{ }^{\circ} \mathrm{C}$. Thin, nine $\mathrm{cm}$ electrode wicks were prepared in $0.01 \mathrm{M}$ acetic acid for the anode and IEF 
cathode solution (Thermo Fisher Scientific) for the cathode. Protein samples were prepared in deionized water with a concentration of approximately $2 \mathrm{mg} / \mathrm{mL}$ and a target total protein load of $10 \mu \mathrm{g}$ per lane. The outer two lanes of each gel contained Bio-Rad IEF Standards 4.45-9.6 (Hercules, CA, USA). The gel was pre-focused for 10 minutes at $1 \mathrm{~W}$, and then run at $1000 \mathrm{~V}$ and $25 \mathrm{~W}$ until the current no longer declined. The gel was stained in Bio-Rad Coomassie Blue G-250 solution, then placed in fixative solution $(36 \% \mathrm{MeOH} v / \mathrm{v}, 6 \%$ trichloroacetic acid w/v, $2 \%$ sulfosalicyclic acid $\mathrm{w} / \mathrm{v})$, and finally placed in destaining solution ( $25 \% \mathrm{EtOH} \mathrm{v} / \mathrm{v}, 9 \%$ glacial acetic acid v/v), with each step lasting for 30 minutes under gentle agitation. After destaining, the gel was removed from solution, placed between blotting paper under weights, and allowed to dry overnight.

\subsubsection{Column Experiments}

A large piece of this work is based on analyzing the chromatographic behavior of columns under varying processing and running conditions. Columns were run on one of several chromatographic workstations depending on the nature of the experiment. Preparative experiments were performed on an ÄKTA Explorer 10 with accompanying Frac-950 fraction collector and P-960 sample pump, or an ÄKTA Pure 25 with accompanying F9-C fraction collector and P9-S sample pump, both systems from GE Healthcare (Piscataway, NJ, USA). Analytical experiments were performed on a Waters e2695 Alliance HPLC (Milford, MA, USA) equipped with several in-line detection modules: UV was monitored by a SpectraSystem UV1000 (Thermo Fisher Scientific, Waltham, MA, USA), refractive index was measured by a Waters 2414 refractive index detector (Milford, MA, USA), and conductivity was monitored by a Waters 430 conductivity detector (Milford, MA, USA). Analytical experiments were also performed on a Waters Acquity H-Class UPLC with built in UV monitor (Milford, MA, USA).

All Nuvia HR-S columns were packed in the lab on an ÄKTA workstation using laboratory scale glass columns $0.5 \mathrm{~cm}$ in diameter (a Tricorn 5/100 with column packing extension tube and an HR 5/5 column were used, both obtained from GE Healthcare, Piscataway, NJ, USA). The 
columns were flow packed with the resin to varying bed heights between 3 and $11 \mathrm{~cm}$ by first preparing an approximately 50\% slurry in $\mathrm{pH} 7$ phosphate buffer with $1 \mathrm{M} \mathrm{NaCl}$ added, pouring a calibrated volume of the slurry in the column, quickly inserting the top adaptor, and then running the mobile phase at $2.5 \mathrm{~mL} / \mathrm{min}$ until the bed was consolidated. The packing quality of all columns was tested by measuring the HETP and asymmetry for a pulse injection of salt. Reduced HETP values and asymmetry factors for salt were $6.0 \pm 1.2$ and $0.97 \pm 0.15$, respectively. The extraparticle void fractions of all columns were obtained from the retention of pulse injections of blue dextran, assuming that it is completely excluded from the resin pores, and were measured to be $0.38 \pm 0.03$.

Isocratic elution and linear gradient elution (LGE) experiments were performed to characterize low protein loading behavior. For these experiments, $100 \mu \mathrm{L}$ injections of purified protein at approximately $2 \mathrm{mg} / \mathrm{mL}$ concentration were loaded onto the column. Isocratic elution experiments were run under non-binding conditions in $10 \mathrm{mM} \mathrm{Na}_{2} \mathrm{HPO}_{4}, 1 \mathrm{M} \mathrm{NaCl}$ at pH 7.0 at flow rates ranging from 0.125 to $3.25 \mathrm{~mL} / \mathrm{min}$, corresponding to reduced velocities between 65 and 2050. The first and second moments of the resulting peaks were obtained by numerical integration and used to calculate the reduced HETP. Proteins were loaded in $10 \mathrm{mM} \mathrm{Na}_{2} \mathrm{HPO}_{4}$, $\mathrm{pH} 7.0$ and eluted with 5, 10, 15, 25, and $40 \mathrm{CV}$ gradients to $10 \mathrm{mM} \mathrm{Na}_{2} \mathrm{HPO}_{4}, \mathrm{pH} 7.0$ with 300 $\mathrm{mM}$ added $\mathrm{NaCl}$.

For the frontal analysis experiments, all protein feeds were prepared with a total protein concentration of $2.0 \pm 0.3 \mathrm{mg} / \mathrm{mL}$, and loaded onto the column for 120 to 380 column volumes, depending on the load buffer $\mathrm{Na}^{+}$concentration and feed composition. For the two-component experiments, fractions were collected in $4 \mathrm{CV}$ increments and analyzed off-line via size exclusion chromatography (see Section 3.2.5). After loading was completed, the column was eluted with $1 \mathrm{M} \mathrm{NaCl}$, and the resulting eluate pool was also analyzed off-line via size exclusion chromatography. Protein recoveries in the elution pool were in excess of $90 \%$ for all experiments. 


\subsubsection{Size Exclusion Chromatography}

Size exclusion chromatography was performed for three different scales and purposes. A HiPrep 26/10 desalting column (GE Healthcare, Piscataway, NJ, USA) was used for bufferexchange on the ÄKTA Pure 25. For this purpose, protein ( $<35 \mathrm{mg} / \mathrm{mL},<12 \mathrm{~mL}$ ) was loaded using a $50 \mathrm{~mL}$ Superloop (GE Healthcare, Piscataway, NJ, USA) at $10 \mathrm{~mL} / \mathrm{min}$ into the desalting column, previously equilibrated with the desired buffer. A Superdex 200 Increase 10/300 GL column (GE Healthcare, Piscataway, NJ, USA) was used to isolate antibody dimer from monomer on the ÄKTA workstations. The protein solution enriched in dimer (see Section 3.1.2) was run at $0.5 \mathrm{~mL} / \mathrm{min}$ in $50 \mathrm{mM} \mathrm{Na}_{2} \mathrm{HPO}_{4}, 150 \mathrm{mM} \mathrm{NaCl}$, pH 6.8 with $0.5 \mathrm{~mL}$ injections of approximately $5 \mathrm{mg} / \mathrm{mL}$ serially injected. The resulting dimer peaks were collected separately from the monomer and the purity was assessed based on analytical SEC. Analytical SEC was run on two different columns in order to differentiate protein size. Using the HPLC system

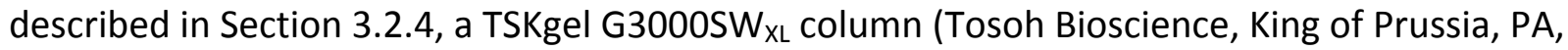
USA) was run isocratically with a mobile phase of $100 \mathrm{mM} \mathrm{Na}_{2} \mathrm{HPO}_{4}, 300 \mathrm{mM} \mathrm{NaCl}$, pH 6.8 at 0.5 or $1.0 \mathrm{~mL} / \mathrm{min}$ with injection volumes of either 50 or $100 \mu \mathrm{L}$ and a 20 -minute run time. Using the UPLC system described in Section 3.2.4, an Acquity UPLC Protein BEH SEC column (200 pore, $1.7 \mu \mathrm{m}$ particles) and accompanying guard column (Waters, Milford, MA, USA) was run isocratically with a mobile phase of $100 \mathrm{mM} \mathrm{Na}_{2} \mathrm{HPO}_{4}, 300 \mathrm{mM} \mathrm{NaCl}, \mathrm{pH} 6.8$ at $0.3 \mathrm{~mL} / \mathrm{min}$ with an injection volume of $5 \mu \mathrm{L}$ and a 7-minute run time.

\subsubsection{Analytical HPLC}

Both reverse phase chromatography (RP) and HPLC cation exchange chromatography were used to characterize the various protein samples. The protein solutions were prepared at 1 $\mathrm{mg} / \mathrm{mL}$ in the respective load buffers, with $5 \mu \mathrm{L}$ injections for the reverse phase experiments, and 10 or $20 \mu \mathrm{L}$ injections for the HPLC CEX experiments. Using the UPLC system described in Section 3.2.4, an Acquity UPLC BEH300 C4 RP column (Waters, Milford, MA, USA) was run with a water/acetonitrile gradient at $0.35 \mathrm{~mL} / \mathrm{min}$. Using the UPLC system described in Section 3.2.4, 
a ProPac WCX-10 CEX column (Thermo Fisher Scientific, Waltham, MA, USA) was run with a 10 $\mathrm{mM} \mathrm{Na}_{2} \mathrm{HPO}_{4}, \mathrm{pH} 7.0 / 10 \mathrm{mM} \mathrm{Na}_{2} \mathrm{HPO}_{4}, 0.25 \mathrm{M} \mathrm{NaCl}, \mathrm{pH} 7.0$ gradient at $1 \mathrm{~mL} / \mathrm{min}$.

\subsubsection{Inverse Size Exclusion Chromatography}

Inverse size exclusion chromatography (iSEC) was used to estimate the pore radius of the resin. The probes were glucose, 4k, 10k, 40k, 70k, 270k, and 670k dextran, and 2000k blue dextran. Each probe was prepared at $5 \mathrm{mg} / \mathrm{mL}$ in $10 \mathrm{mM} \mathrm{Na}_{2} \mathrm{HPO}_{4}, 1 \mathrm{M} \mathrm{NaCl}, \mathrm{pH} 7.0$ and used a $20 \mu \mathrm{L}$ injection volume at $0.5 \mathrm{~mL} / \mathrm{min}$ using the Waters HPLC unit and in-line RI detector described in Section 3.2.4. The system extra-column volume was measured using separate glucose and blue dextran injections with an extra-column mimic in-line, yielding an extra-column volume of $0.380 \pm 0.005 \mathrm{~mL}$. A Nuvia HR-S column packed as described in Section 3.2.4 was attached, and each probe was injected. The peak retentions were measured based on both the first moment and the retention at the peak maximum.

\subsubsection{Dynamic Light Scattering}

Dynamic light scattering (DLS) experiments were performed using the Waters HPLC unit with inline UV described in 3.2.4 and an in-line dynamic light scattering module (DynaPro NanoStar and miniDAWN TREOS, Wyatt Technologies, Santa Barbara, CA, USA). For these experiments, protein samples were run over the TSKgel G3000SW $\mathrm{XL}_{\mathrm{X}}$ column described in Section 3.2.5. In order to achieve sufficient autocorrelation functions, high protein concentrations were necessary, requiring $100 \mu \mathrm{L}$ injection volumes with samples prepared at $5 \mathrm{mg} / \mathrm{mL}$ and with a flow rate of $0.5 \mathrm{~mL} / \mathrm{min}$. Autocorrelation function decay curves were analyzed in ASTRA V 5.3.4.20 software (Wyatt Technologies, Santa Barbara, CA, USA). 


\subsubsection{Equilibrium Adsorption Isotherms}

Adsorption isotherms were obtained for a range of salt concentrations by mixing known amounts of resin with protein solutions of varying initial concentration. The resin was first washed and equilibrated with each load buffer and then filtered for 15 min to remove any interstitial liquid using Corning ${ }^{\circledR}$ Spin- $X^{\circledR}$ microfiltration tubes with an Eppendorf Minispin ${ }^{\circledR}$ bench-top centrifuge operated at 5000 RPM. Small quantities of the filtered resin (10-30 mg) were added to $1.5 \mathrm{~mL}$ centrifuge tubes containing $1.5 \mathrm{~mL}$ of protein solution. The tubes were then sealed and rotated gently overnight ( $~ 12 \mathrm{hr}$ ) before being centrifuged at 5000 RPM for 5 min to separate the resin from supernatant. The supernatant UV280 absorbance was measured in either a 1-cm optical path disposable cuvette on a DU ${ }^{\circledR} 640$ spectrophotometer (BeckmanCoulter, Indianapolis, IN, USA) or as a micro-aliquot on a NanoDrop 2000c UV-vis spectrophotometer (Thermo Fisher Scientific, Waltham, MA, USA). The total protein bound was determined by mass balance, with the resin mass converted to volume using the hydrated particle density, previously determined in Section 3.2.2. The bound protein concentration is given by:

$$
\langle\bar{q}\rangle=\frac{V}{M_{w} / \rho_{r}}\left(C_{0}-C\right)
$$

where $V$ is the volume of protein solution, $\rho_{r}$ is the resin density (measured as described in Section 3.2.2), $M_{w}$ is the mass of centrifuged resin, $C_{0}$ is the initial protein concentration, and $C$ is the supernatant protein concentration. For two-component isotherms, the feed and supernatant were analyzed by SEC as described in Section 3.2.5 or by HPLC CEX as described in Section 3.2.6 in order to fully define the mass balances.

\subsubsection{Confocal Laser Scanning Microscopy}

Confocal laser scanning microscopy (CLSM) was used to observe the intraparticle distribution of antibody monomer and dimer during transient adsorption, both individually and in mixtures. For this purpose, monomer and purified dimer samples were fluorescently labeled with aminereactive dyes Rhodamine $\operatorname{Red}^{\mathrm{TM}}-\mathrm{X}$ and $\mathrm{Green}^{\mathrm{TM}}-\mathrm{X}$, respectively, by incubating $1 \mathrm{~mL}$ of solutions 
containing $8 \mathrm{mg} / \mathrm{mL}$ protein in $500 \mathrm{mM} \mathrm{NaHCO}_{3}, \mathrm{pH} 8.5$ buffer with each reactive dye in darkness for $1 \mathrm{~h}$ following the method in Tao, et al. (2011) and Perez-Almodóvar, et al (2012). The dye-to-protein molar ratio was 1:3 for the monomer and 1:6 for the dimer, which resulted in average molar labeling ratios of 0.24 and 0.16 for the monomer and dimer, respectively, determined spectrophotometrically based on the dye extinction coefficients at 571 and $503 \mathrm{~nm}$

for Rhodamine $\operatorname{Red}^{\mathrm{TM}}-\mathrm{X}$ and Rhodamine $\mathrm{Green}^{\mathrm{TM}}-\mathrm{X}$ protein conjugates, respectively. A PD 10 desalting column (GE Healthcare, Piscataway, NJ, USA) was used to remove unreacted dye.

Labeled protein was added to a solution of unlabeled protein in a $1 / 40$ ratio resulting in final labeling ratios of $1 / 166$ and $1 / 250$ for the monomer and dimer, respectively, with a total protein concentration of approximately $2 \mathrm{mg} / \mathrm{mL}$. At time zero, a small amount of filtered resin $(<5 \mathrm{mg})$, prepared in the same manner as the isotherm measurements of Section 3.2.9, was added to $5 \mathrm{~mL}$ of the labeled/unlabeled protein solution and gently agitated. At varying times, $300 \mu \mathrm{L}$ samples were taken from the mixture, placed in Spin- $X^{\circledR}$ microfiltration tubes, and spun for $30 \mathrm{~s}$ to remove excess supernatant with an Eppendorf Minispin ${ }^{\circledR}$ centrifuge at 13400 RPM. The resin beads held in the filter basket were then re-suspended in a solution containing $50 \%$ $(w / w)$ sucrose in the load buffer and then quickly added onto a slide for imaging. Sucrose was used to match the refractive index of the resin particles, allowing imaging with little or no decay in fluorescence intensity (Tao, Carta, et al. 2011; Pérez-Almodóvar et al. 2012; Pérez-Almodóvar et al. 2011). Imaging was performed with a Zeiss LSM 510 microscope with a Plan-Apochromat $63 \times 1.4$ NA oil objective (Carl Zeiss Microlmaging, LLC, Thornwood, NY, USA) using a 561 nm diode-pumped solid state laser for the monomer and a $488 \mathrm{~nm}$ argon gas laser for the dimer. All data were analyzed using ImageJ software (Schneider et al. 2012).

\subsubsection{Biolayer Interferometry}

The experiments utilizing biolayer interferometry (BLI) were performed on BLItz and Octet Red96 systems from Pall ForteBio (Menlo Park, CA, USA). The single-channel BLItz system was used preliminarily for concentration range scouting and probe testing. The multi-channel Octet 
system was used for high-throughput data collection. The sample probes used on the system were amine-reactive $2^{\text {nd }}$ generation (AR2G) biosensors, also from Pall ForteBio. These probes possess a carboxy-terminated surface ligand intended for the reaction-mediated covalent coupling of amine groups. An AR2G reagent kit was purchased from Pall ForteBio for attempts to functionalize the surface with a strong cation exchange ligand.

Protein solutions were prepared by buffer exchange into $10 \mathrm{mM} \mathrm{Na}_{2} \mathrm{HPO}_{4}$ at $\mathrm{pH} 7.0$ with different $\mathrm{NaCl}$ concentrations using a HiPrep 26/10 desalting column (as described in Section 3.2.5), and then diluted to $0.5-1.0 \mathrm{mg} / \mathrm{mL}$ with the same buffer. All buffers and proteins used were $0.22 \mu \mathrm{m}$ filtered prior to use. For the BLI measurements, $200 \mu \mathrm{L}$ samples were aliquoted into opaque $0.5 \mathrm{~mL}$ polypropylene tubes for the BLItz system and into opaque 96-well trays for the Octet system. For both the BLItz and Octet, a stock protein solution at high concentrations $(0.5-1.0 \mathrm{mg} / \mathrm{mL})$ was used as a saturation control. For lower concentration experiments, the stock solution was diluted 100x with the corresponding buffer, and then serially diluted 1:1 with buffer to achieve the desired concentration range.

All experiments followed a similar protocol, shown in Figure 3.1. Prior to use, all AR2G probes were hydrated in the experiment buffer for at least $10 \mathrm{~min}$. For single component measurements (Fig. 3.1a), each run comprised a two-minute baseline step, a protein association step of varying length depending on the total $\mathrm{Na}^{+}$concentration, a five-minute dissociation step, and a two-minute strip step. For sequential adsorption measurements (Fig. 3.1b), the same protocol was followed, but with an additional protein association and dissociation step prior to the strip step. For each experiment, the hydration, baseline, association, and dissociation steps each had the same buffer compositions, unless otherwise noted. The strip step buffer was $10 \mathrm{mM} \mathrm{Na}_{2} \mathrm{HPO}_{4}$ with $1 \mathrm{M}$ added $\mathrm{NaCl}$ at $\mathrm{pH}$ 7.0. For experiments performed on the Octet, reference wells with no protein present during the association phase(s) were used as controls. Results from the BLItz and Octet systems were captured using BLItz Pro v.1.1.1.33 and Octet Data Acquisition Software v.8.2.0.9, both from Pall ForteBio. 

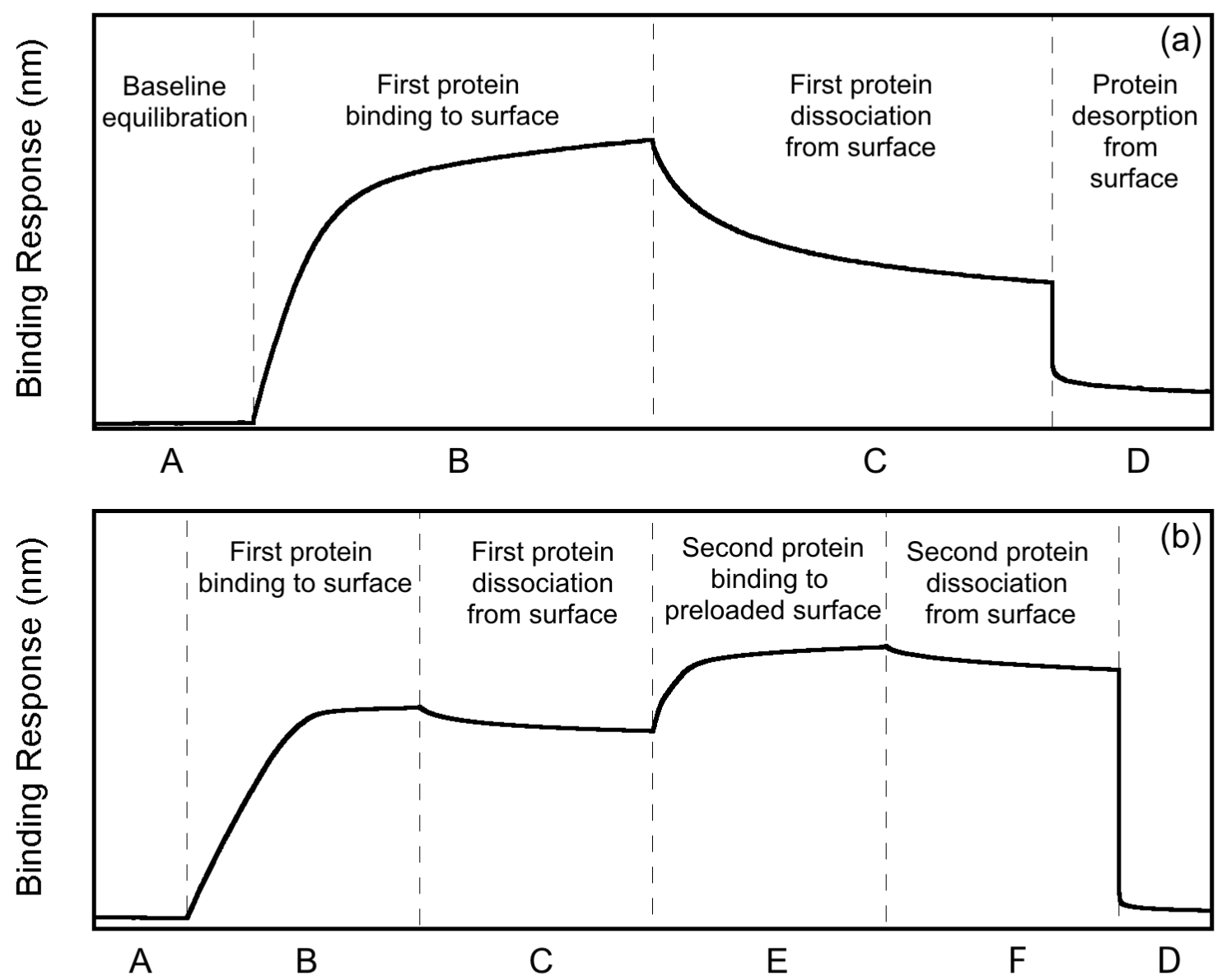

Figure 3.1. Sensorgram schematic for (a) single component protein binding experiments and (b) sequential protein binding experiments. A is a baseline equilibration in load buffer, $B$ is the first protein binding to a clean surface, $C$ is the first protein dissociating from the surface, $D$ is a protein desorption in high salt, $\mathrm{E}$ is the second protein binding to a preloaded surface, and $\mathrm{F}$ is the second protein dissociating from the surface. 


\section{Resin Characterization}

\subsection{Microscopy}

The Nuvia HR-S particle size distribution was determined as described in Section 3.2.1. Figure 4.1a shows a representative section of one of the micrographs. From each individually micrographed particle, the volume-averaged particle diameter was calculated using: $\bar{d}_{p}=\sum_{i} f_{i} d_{p, i}$

where $f_{i}$ is the volume fraction of an individual particle, and $d_{p, i}$ is the diameter of an individual particle. The volume-averaged diameter for Nuvia HR-S was $52.6 \pm 5.7 \mu \mathrm{m}$ based on 160 individually imaged resin particles. The particles are relatively mono-dispersed compared to other similarly sized resins (Wu et al. 2013; Pérez-Almodóvar et al. 2011), with the measured distribution narrower than what is claimed by the manufacturer. Figure $4.1 \mathrm{~b}$ shows the histogram of particle diameters of the imaged resin particles.

Additionally, Nuvia HR-S particles were imaged via Transmission Electron Microscopy (TEM) as described in Pérez-Almodóvar et al. (2011). Two images of different magnification are shown in Figure 4.2. Each image is taken near the particle surface to illustrate the curvature of the spherical particles. The darker sections in each image are the solid base matrix while the light gray areas throughout the remainder are the embedding resin used to fix the samples. From the images, Nuvia HR-S has an open macroporous structure, with some of the interior pores measuring several hundred nanometers in size.

\subsection{Inverse Size Exclusion Chromatography}

A packed column of Nuvia-HR S was analyzed via inverse size exclusion chromatography (iSEC) as described in Section 3.2.7. Table 4.1 lists the nominal probe molecular weights and the correlated hydrodynamic radii in nm using the correlation of Hagel et al. (1996), given by:

$r_{H}=0.027\left(M_{r}\right)^{0.498}$

where $r_{H}$ is the hydrodynamic radius in $\mathrm{nm}$, and $M_{r}$ is the molecular weight in Daltons. 

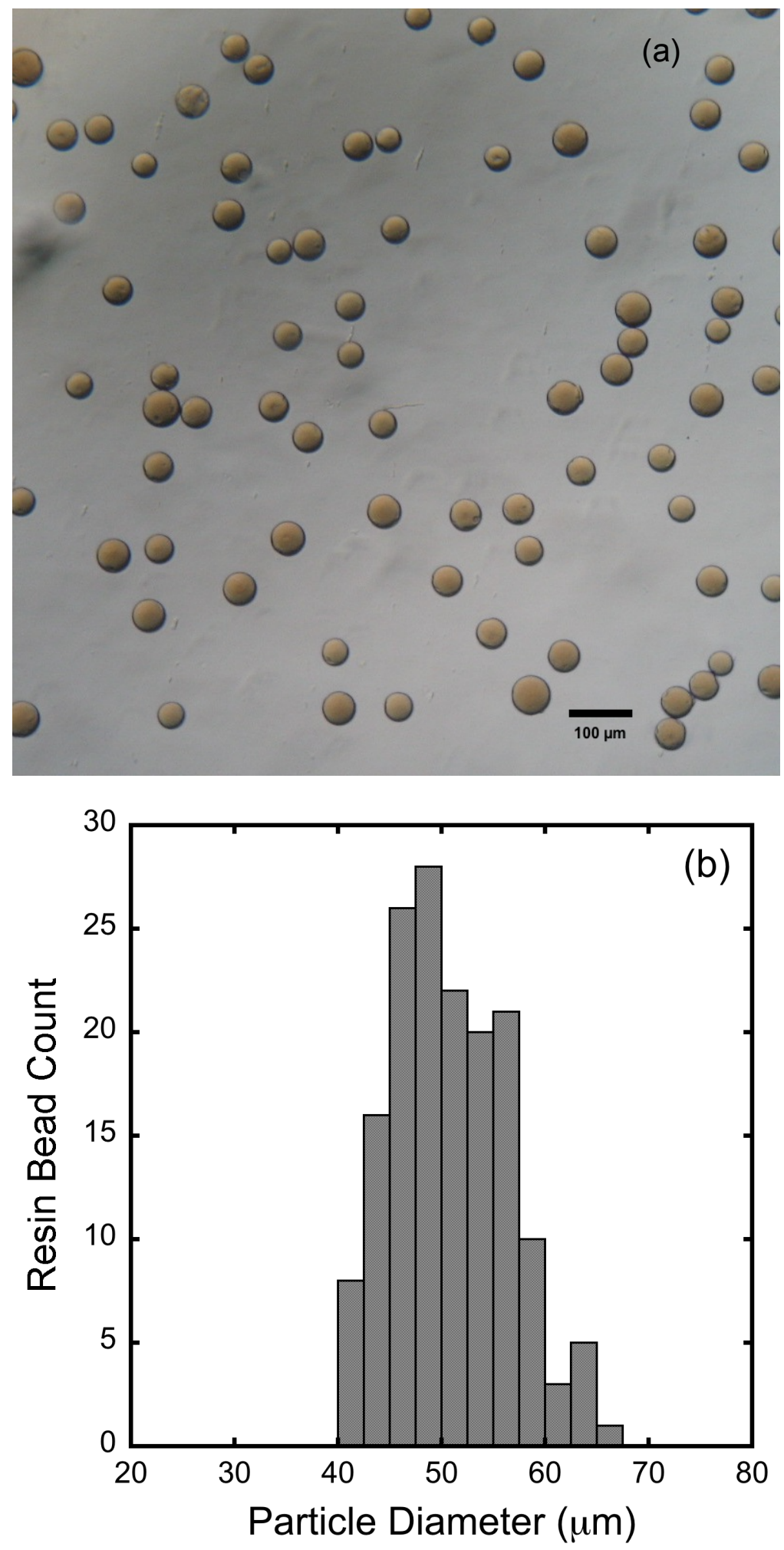

Figure 4.1. (a) Representative micrograph image and (b) particle size distribution for Nuvia-HR S taken from microphotographs at 10x magnification. 

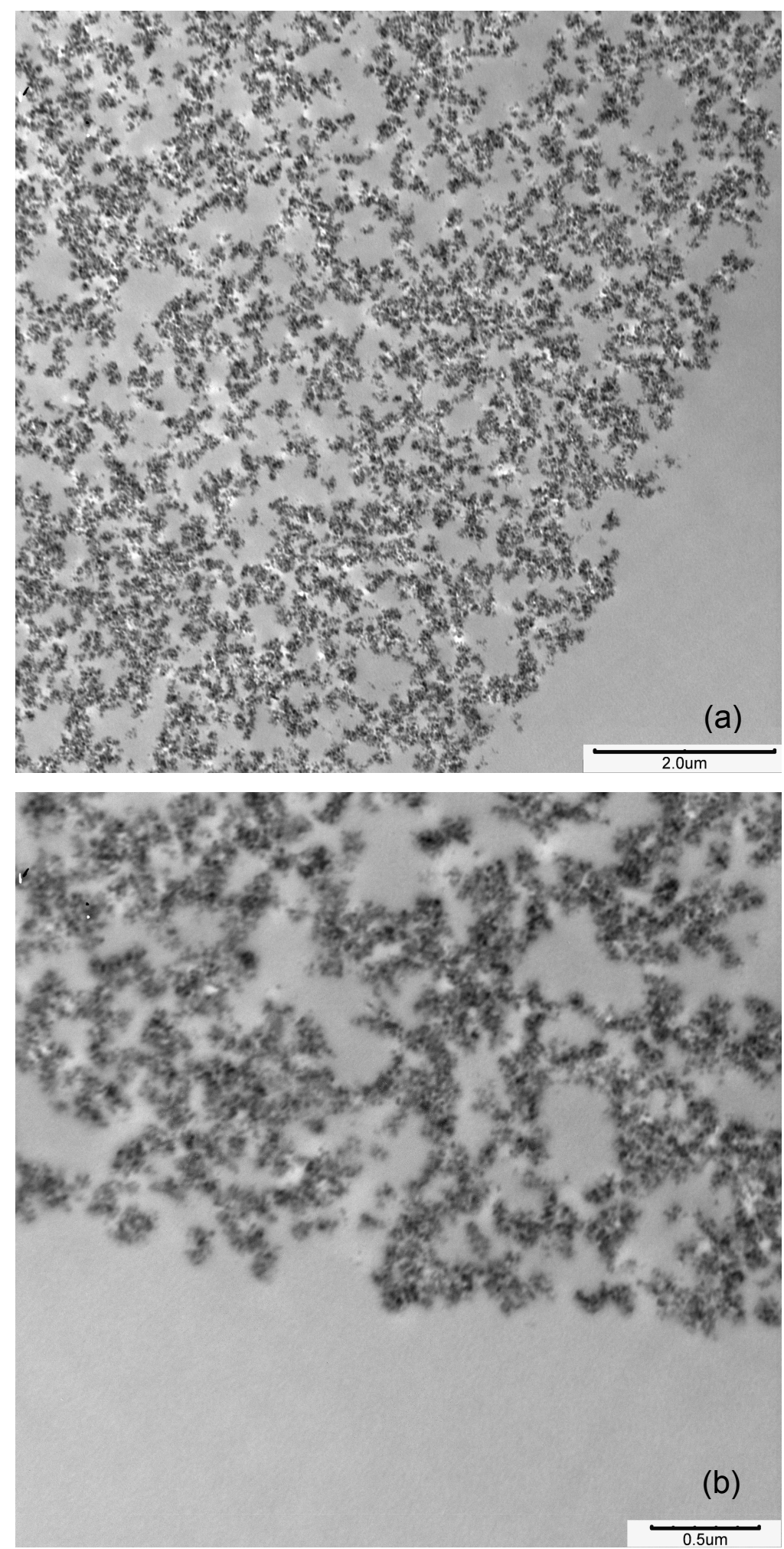

Figure 4.2. Sample TEM images of Nuvia HR-S at (a) 8000x and (b) 20000x magnification. 
Chromatograms of the refractive index signal for the various dextran probes are shown in Fig. 4.3, along with the calculated extra-column porosity. The peak retention was calculated for each peak by both the first moment and the retention at peak maximum. The retention volumes are adjusted by subtracting the extra-column retention volume in the system, determined from a glucose and high molecular weight dextran sample injection with a zerovolume column mimic.

The experimental distribution coefficient was calculated by:

$$
K_{D}=\frac{V_{R} / V_{C}-\varepsilon}{1-\varepsilon}
$$

where $V_{R}$ is the adjusted retention volume from the first moment or peak maximum, $V_{C}$ is the column volume, and $\varepsilon$ is the column porosity. The values of $K_{D}$ using both the first moment and peak retention at maximum signal are reported in Table 4.1. The values show good agreement for both methods for smaller probes, but begin to differ as the size of the probe increases. This can be seen visually in Fig. 4.3 where the 270 and 670 dextran probes show significant tailing, likely due to a higher polydispersity in these samples. These distribution coefficients are then fitted to existing models related to pore architecture. One common model is the single cylindrical pore approximation, which is given by (Striegel et al. 2009):

$$
K_{D}=\varepsilon_{p}\left(1-\frac{r_{H}}{r_{\text {pore }}}\right)^{2}
$$

where the parameters $\varepsilon_{p}$, the intraparticle resin porosity, and $r_{p o r e}$, the pore radius, are regressed parameters. Using the moment method calculations for $K_{D}$ and a linearized form of this model yields $\varepsilon_{p}=0.765 \pm 0.037$ and $r_{\text {pore }}=54.8 \pm 7.3 \mathrm{~nm}$. The same analysis using the peak maximum calculations yields $\varepsilon_{p}=0.784 \pm 0.041$ and $r_{\text {pore }}=41.6 \pm 3.0 \mathrm{~nm}$. In both instances, the reported errors are regression errors. The moment method calculated value of $55 \mathrm{~nm}$ is in good agreement with previously reported pore radii for resins with a similar architecture and backbone (Hunter \& Carta 2000). 


\begin{tabular}{|c|c|c|c|c|}
\hline Probe & $\begin{array}{c}\text { Nominal } M_{r} \\
\text { (Da) }\end{array}$ & $\begin{array}{c}\text { Correlated } r_{H} \\
\mathbf{( n m})\end{array}$ & $\begin{array}{c}\text { First Moment } \\
K_{D}\end{array}$ & $\begin{array}{c}\text { Peak Maximum } \\
K_{D}\end{array}$ \\
\hline Glucose & 180 & 0.359 & 0.859 & 0.881 \\
\hline Dextran 4 & 4,000 & 1.68 & 0.746 & 0.764 \\
\hline Dextran 10 & 10,000 & 2.65 & 0.660 & 0.673 \\
\hline Dextran 40 & 40,000 & 5.29 & 0.575 & 0.575 \\
\hline Dextran 70 & 70,000 & 6.99 & 0.530 & 0.510 \\
\hline Dextran 270 & 270,000 & 13.7 & 0.407 & 0.328 \\
\hline Dextran 670 & 670,000 & 21.5 & 0.316 & 0.127 \\
\hline
\end{tabular}

Table 4.1. Inverse size exclusion chromatography probe molecular weights, and correlated hydrodynamic radii by Hagel et al. (1996), and measured distribution coefficients 


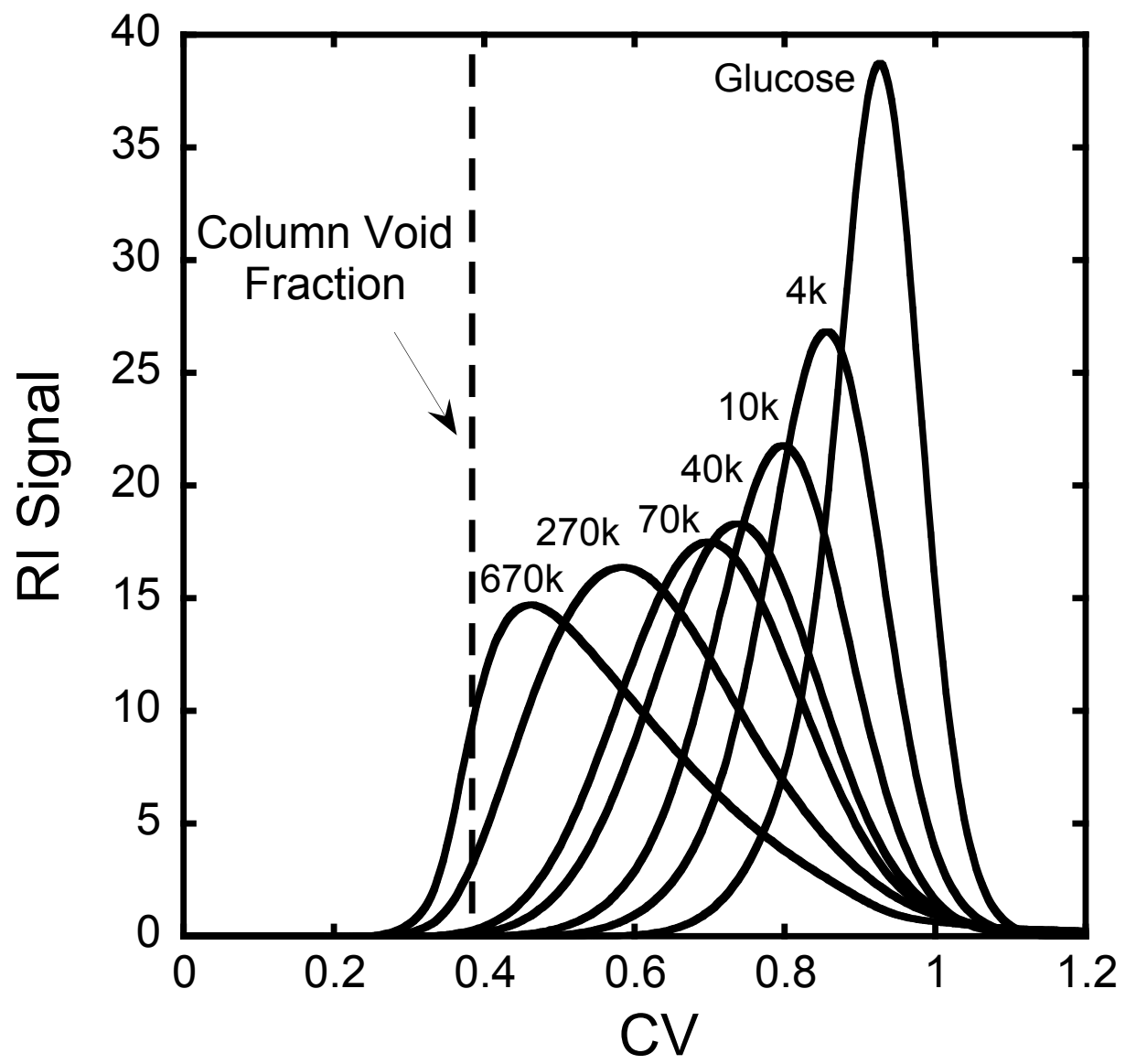

Figure 4.3. Inverse size exclusion chromatography peaks for glucose and dextran probes on Nuvia HR-S column 


\section{Protein Characterization}

\subsection{Analytical Chromatography}

Various analytical chromatographic techniques were performed on the antibody samples used throughout this work. Figure 5.1 shows analytical chromatograms of the reverse phase response and HPLC cation exchange response of the four antibodies provided by MedImmune (Section 3.1.2). The reverse phase response in Fig. 5.1a measures the hydrophobicity of the mAbs, with mAb B being the most hydrophobic. Figure $5.1 \mathrm{~b}$ shows a measure of the electrostatic response of the mAbs, with mAb D having the strongest retention, and mAbs $B$ and $\mathrm{C}$ having very similar retention. Additionally, Fig. $5.1 \mathrm{~b}$ reveals a number of charge variant species for the various mAbs, with multiple notable shoulders for mAbs $A$ through $C$, and a large second peak (approximately 10\%) for mAb D. The elution order observed on the analytical cation exchange column does not necessarily ensure that this order of retention will be preserved for other cation exchange resins.

\subsection{Dynamic Light Scattering}

Dynamic light scattering (DLS) experiments were performed for each of the proteins as described in Section 3.2.8. In light scattering experiments, detectors measure the autocorrelation function of the intensity of scattered light given by (Frisken 2001):

$g^{(2)}(\tau)=\frac{\langle I(t) I(t+\tau)\rangle}{\langle I(t)\rangle^{2}}$

where $I(t)$ is the scattered light intensity, $I(t+\tau)$ is the scattered light intensity after a delay time, $\tau$, and the brackets denote an averaging over $t$. Using the method of cumulants (Koppel 1972) and assuming a single-decay rate as would be expected for a monodisperse solution, the autocorrelation function can be fit using (Frisken 2001):

$g^{(2)}(\tau)=B+\beta \exp (-2 \Gamma \tau)$

where $B$ is a baseline that should equal 1 at long times, $\beta$ is an experimental parameter dependent on the system geometry, and $\Gamma$ is the decay rate, which is equal to $D_{0} q^{2}$, where $D_{0}$ 
is the protein diffusivity in solution, and $q$ is the magnitude of the scattering wave vector. The difference between the incident and scattered light wave vectors is given by:

$q=\frac{4 \pi n}{\lambda_{0}} \sin \left(\frac{\theta}{2}\right)$

where $n$ is the refractive index of the solution, $\lambda_{0}$ is the laser wavelength in a vacuum, and $\theta$ is the scattering angle of the instrument. Experimentally, the DLS instrument extracts the protein diffusivity from the fitted decay rate $(\Gamma)$ for a given autocorrelation function. This diffusivity is then converted to a hydrodynamic radius using the well-known Stokes-Einstein relation, given by:

$$
D_{0}=\frac{k_{B} T}{6 \pi \eta r_{H}}
$$

where $k_{B}$ is the Boltzmann constant, $T$ is temperature, $\eta$ is the solution dynamic viscosity, and $r_{H}$ is the hydrodynamic radius of an equivalent spherical particle. Because of the globular nature of the proteins used here, this is a reasonable assumption.

The DLS experiments were run with an in-line HPLC SEC column to separate the pure protein peaks by size, with each point along the peak having its own distinct autocorrelation function. Figure 5.2a shows the SEC chromatograms of the mAb monomers with their hydrodynamic radius at each point along the peak as extracted from the corresponding autocorrelation function. All of the mAbs emerge at the same time with the exception of $m A b B$, which elutes slightly later. Despite this later elution, all four mAb monomers have statistically similar hydrodynamic radii. The late eluting behavior of $\mathrm{mAb} B$ may be related to its similarly different behavior on the reverse phase column (Fig. 5.1a), with its increased hydrophobicity possibly resulting in a slight interaction with the SEC column matrix. Figure 5.2b shows the SEC chromatogram of the dimer-enriched sample of mAb D (see Section 3.1.2). There is a large early eluting peak corresponding to the dimer, followed by the later eluting monomer. The hydrodynamic radii from the DLS measurements of these peaks corroborate this interpretation of the SEC data. The SEC data and hydrodynamic radii of each species are summarized in Table 5.1. 

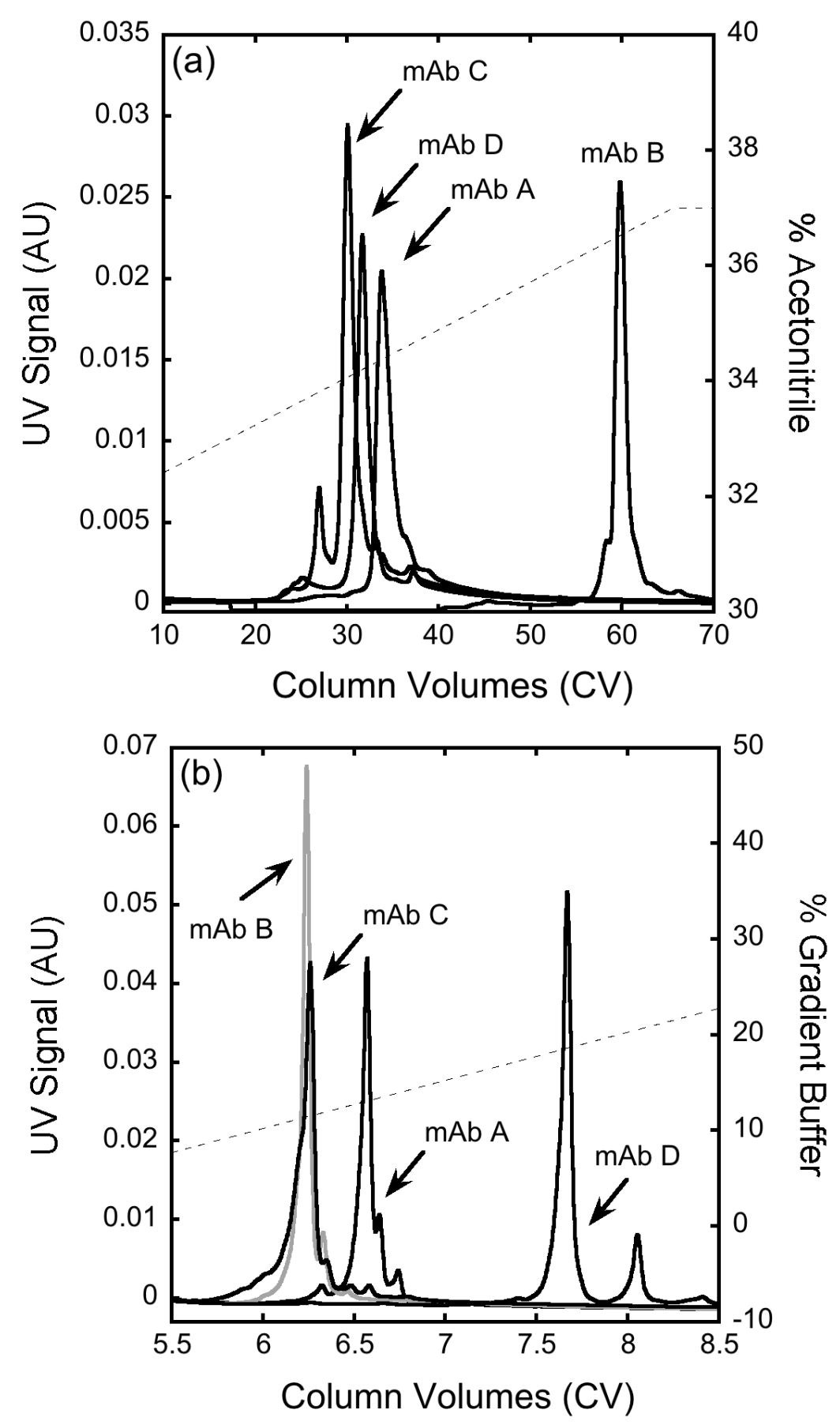

Figure 5.1. Analytical HPLC chromatograms of mAbs A through D on (a) C4 reverse phase column and (b) WCX-10 CEX column. MAb B chromatogram in (b) has been lightened to allow distinction from $\mathrm{mAb} C$ 

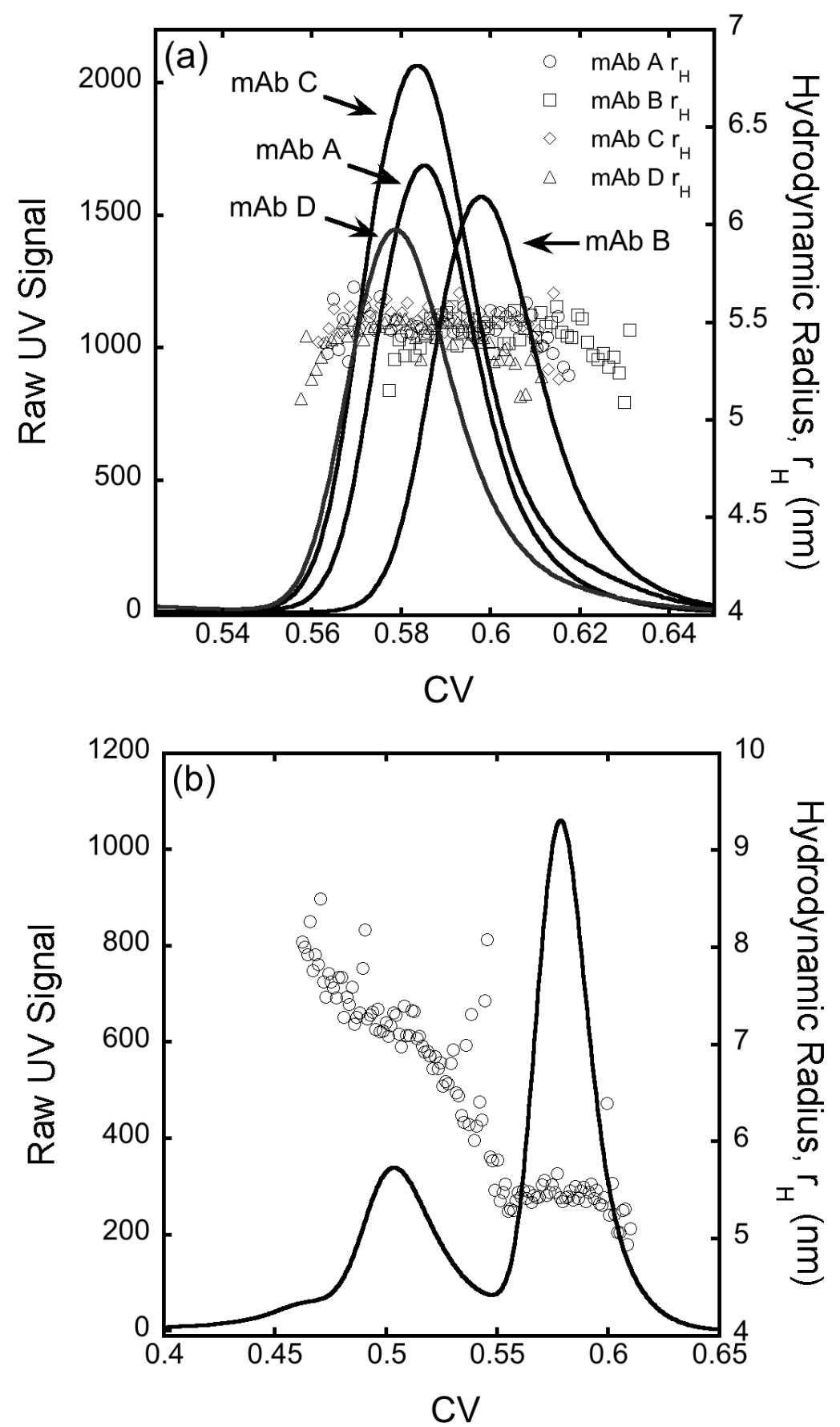

Figure 5.2. Analytical SEC chromatograms of (a) mAbs A-D, and (b) mAb D dimer-rich sample. $r_{H}$-values for each species are reported in Table 5.1 


\subsection{Isoelectric Focusing}

Isoelectric focusing gels were run as described in Section 3.2.3. Figure 5.3 shows a greyscale image of a gel, with standards in lane 1, and mAbs A through D in lanes 2 through 5 , respectively. To extract the $\mathrm{pl}$ for each protein, a greyscale intensity plot was taken of each lane. The pl of each standard in lane 1 was matched to its intensity maximum, and then plotted vs. its distance along the lane. This plot was then fit with a standard curve in order to allow interpolation of $\mathrm{pl}$ for any distance traveled in the lane. The intensity maximum corresponding to each of the four mAb monomers was then fit using this standard curve to extract the corresponding $\mathrm{pl}$. All of the mAb monomers showed very similar pls, with $\mathrm{mAb} C$ being the lowest, and $\mathrm{mAb} D$ being the highest. Each mAb also displays some faint bands near the maximum intensity, indicating the likely presence of some minor charge variants, as can be seen in the HPLC CEX profiles in Fig. 5.1b.

Figure 5.4 shows a greyscale image of a gel, with standards in lane 1, mAb D in lane 2, and a pure mAb D dimer in lane 3, isolated from the mAb D dimer enriched stock via the methods described in Section 3.2.5. Despite some smearing of the mAb D dimer lane, the monomer and dimer form of mAb D both appear to have the same pl. The pl for all of the protein samples is summarized in Table 5.1. 
pH 9.6

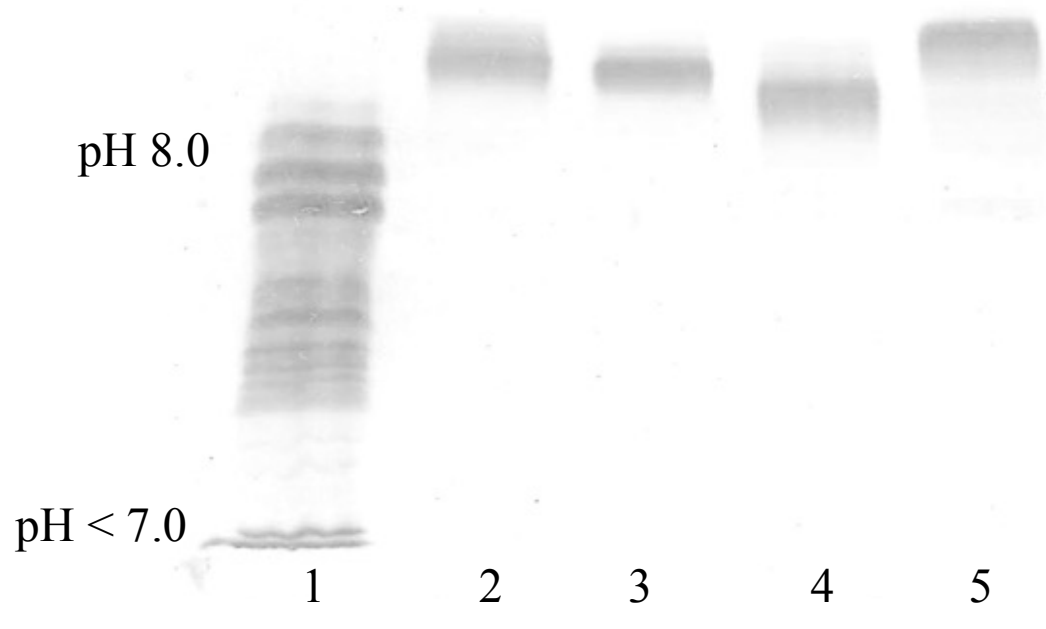

Figure 5.3. IsoGel ${ }^{\circledR}$ Agarose pH 7-11 IEF Plate (Lonza Inc., Rockland, ME, USA) of mAb monomers A through D. Lane 1 contains Broad Range pl 4.45-9.6 IEF Standards (Bio-Rad Laboratories Inc., Hercules, CA, USA). Lane 2 through 5 contains mAb A through D monomers, respectively 
pH 9.6

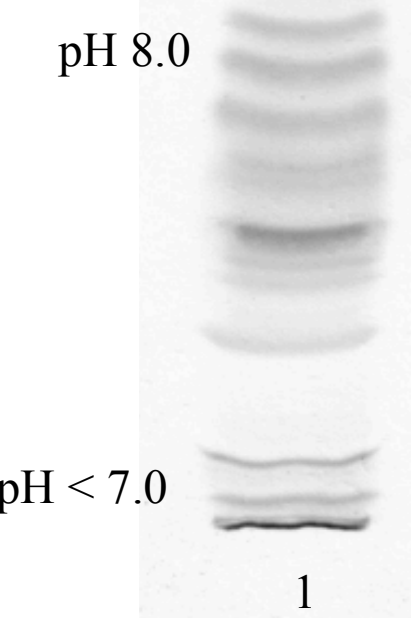

2

3

Figure 5.4. IsoGel ${ }^{\circledR}$ Agarose $\mathrm{pH}$ 7-11 IEF Plate (Lonza Inc., Rockland, ME, USA) of mAb D monomer and dimer samples. Lane 1 contains Broad Range pl 4.45-9.6 IEF Standards (Bio-Rad Laboratories Inc., Hercules, CA, USA). Lane 2 contains mAb D monomer sample. Lane 3 contains mAb D dimer sample 


\begin{tabular}{|c|c|c|c|c|c|c|}
\hline $\begin{array}{c}\text { Protein } \\
\text { Sample }\end{array}$ & $\begin{array}{c}\text { Extinction } \\
\text { Coefficient } \\
(\mathrm{mL} / \mathrm{mg} \mathrm{cm})\end{array}$ & $\begin{array}{c}\% \\
\text { Monomer } \\
\text { by SEC }\end{array}$ & $\begin{array}{c}\% \text { High MW } \\
\text { Species by } \\
\text { SEC }\end{array}$ & $\begin{array}{c}\text { pl from } \\
\text { Isoelectric } \\
\text { focusing gel }\end{array}$ & $\begin{array}{c}D_{0} \text { from } \\
\text { DLS }\left(\times 10^{-7}\right. \\
\left.\mathrm{cm}^{2} / \mathrm{s}\right)\end{array}$ & $\begin{array}{c}r_{H} \text { from DLS } \\
\text { measurements } \\
(\mathrm{nm})\end{array}$ \\
\hline $\mathrm{mAb} \mathrm{A}$ & $1.40^{\mathrm{a}}$ & 99.3 & 0.7 & 8.5 & 3.9 & $5.5 \pm 0.1$ \\
\hline $\mathrm{mAb} \mathrm{B}$ & $1.47^{\mathrm{a}}$ & 99.5 & 0.5 & 8.5 & 4.0 & $5.4 \pm 0.1$ \\
\hline $\mathrm{mAb} \mathrm{C}$ & -- & 98.3 & 1.1 & 8.4 & 3.9 & $5.5 \pm 0.1$ \\
\hline $\mathrm{mAb} \mathrm{D}$ & $1.33^{\mathrm{a}}$ & 97.0 & 2.8 & 8.6 & 4.0 & $5.4 \pm 0.1$ \\
\hline $\begin{array}{c}\mathrm{mAb} D \\
\text { dimer }\end{array}$ & $1.33^{\mathrm{b}}$ & 67.7 & 32.3 & 8.6 & 3.0 & $7.2 \pm 0.6$ \\
\hline
\end{tabular}

Table 5.1. Protein properties and characterization data for mAbs A-D and mAb D dimer

${ }^{a}$ Data provided by MedImmune

${ }^{b}$ Assumed extinction coefficient based on monomeric species 


\section{Characterization of Single Component Adsorption Equilibrium and Kinetics of Antibody Monomer and Dimer on Nuvia HR-S}

This chapter investigates the single component adsorption equilibrium and kinetics behavior of mAb D and its soluble dimer on Nuvia HR-S. Moving forward, it should be assumed that any reference to a mAb monomer and its dimer is referring to $m A b D$, unless otherwise noted.

Evaluating the behavior of the monomer-dimer system required isolation of the antibody dimer from the feedstock discussed in Section 3.1.2. This was done via serial injections on a preparative SEC column, as described in Section 3.2.5. Figure 6.1 shows the results of that separation, with Fig. 6.1a showing the original sample, and Fig. 6.1b showing the highly purified sample containing predominantly dimer (purity > 93\%) and pure monomer for comparison.

\subsection{Retention Behavior at Low Protein Loadings}

Figure 6.2 shows representative linear gradient elution (LGE) chromatograms with different gradient slopes (see Section 3.2.4) for the purified monomer and dimer samples. An impurity present in the monomer sample is partially resolved using the shallower gradients and elutes at the same conductivity as that of the purified dimer. This is likely a small amount of the dimer initially present in the sample, as well as the charge variant seen in Fig 5.1b. As seen in Fig. 6.2, the conductivity at elution, shown by the thick solid lines, decreases as the gradient slope increases and this decrease is more pronounced for the monomer.

According to the stoichiometric displacement model (Kopaciewicz et al. 1983), the protein retention factor as a function of sodium ion concentration can be expressed as:

$k^{\prime}=k_{\infty}^{\prime}+A\left(C_{N a^{+}}\right)^{-z}$

where $k_{\infty}^{\prime}$ is the protein retention factor for non-binding conditions, $z$ is the effective binding charge, and $A$ is a constant, different for each protein, that depends on the resin charge density. The following relationship is obtained between the normalized gradient slope, 

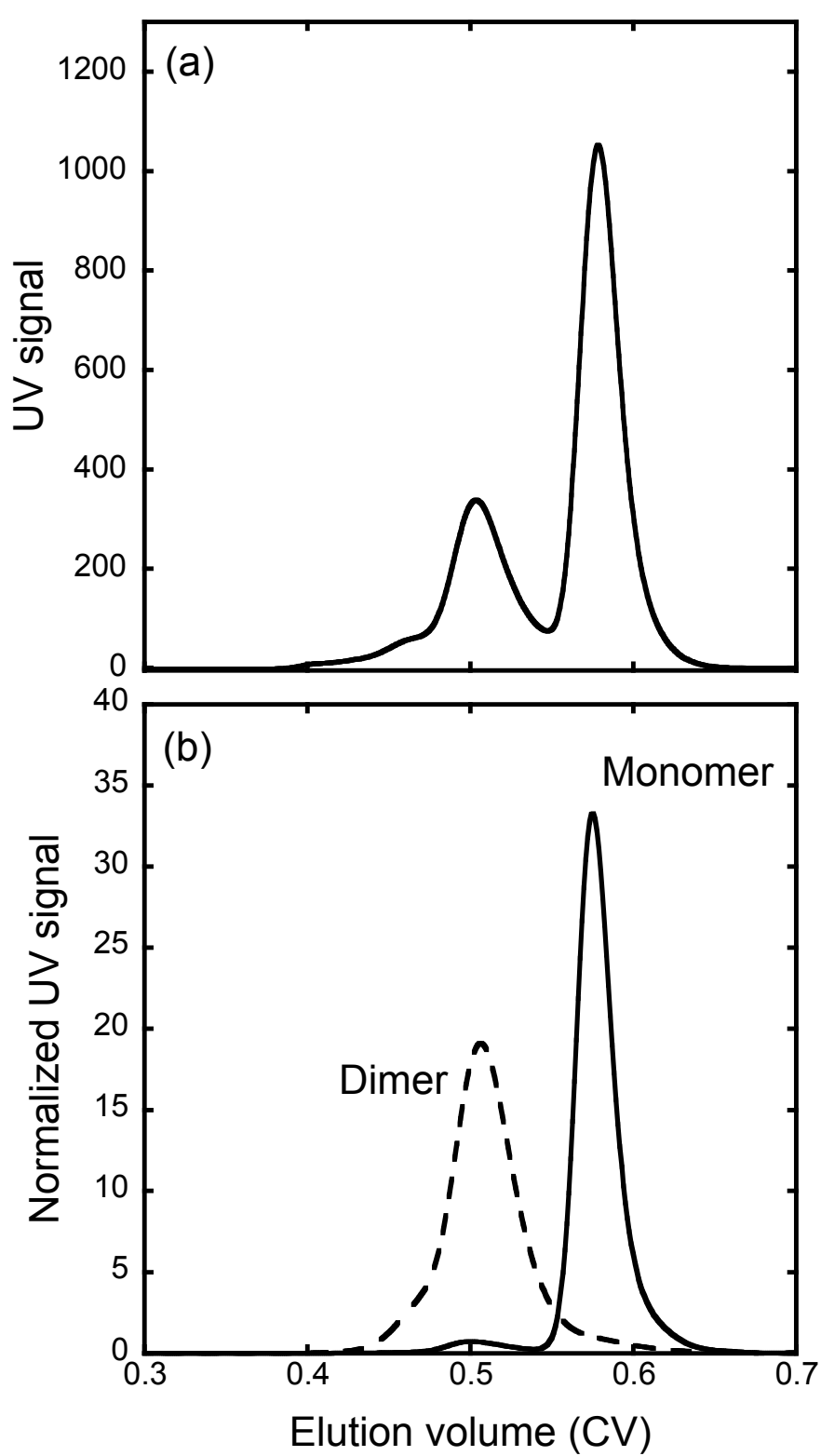

Figure 6.1. Analytical SEC of (a) dimer-rich sample and (b) purified monomer and purified dimer samples. UV signal in (b) is normalized to yield the same peak area for both species 

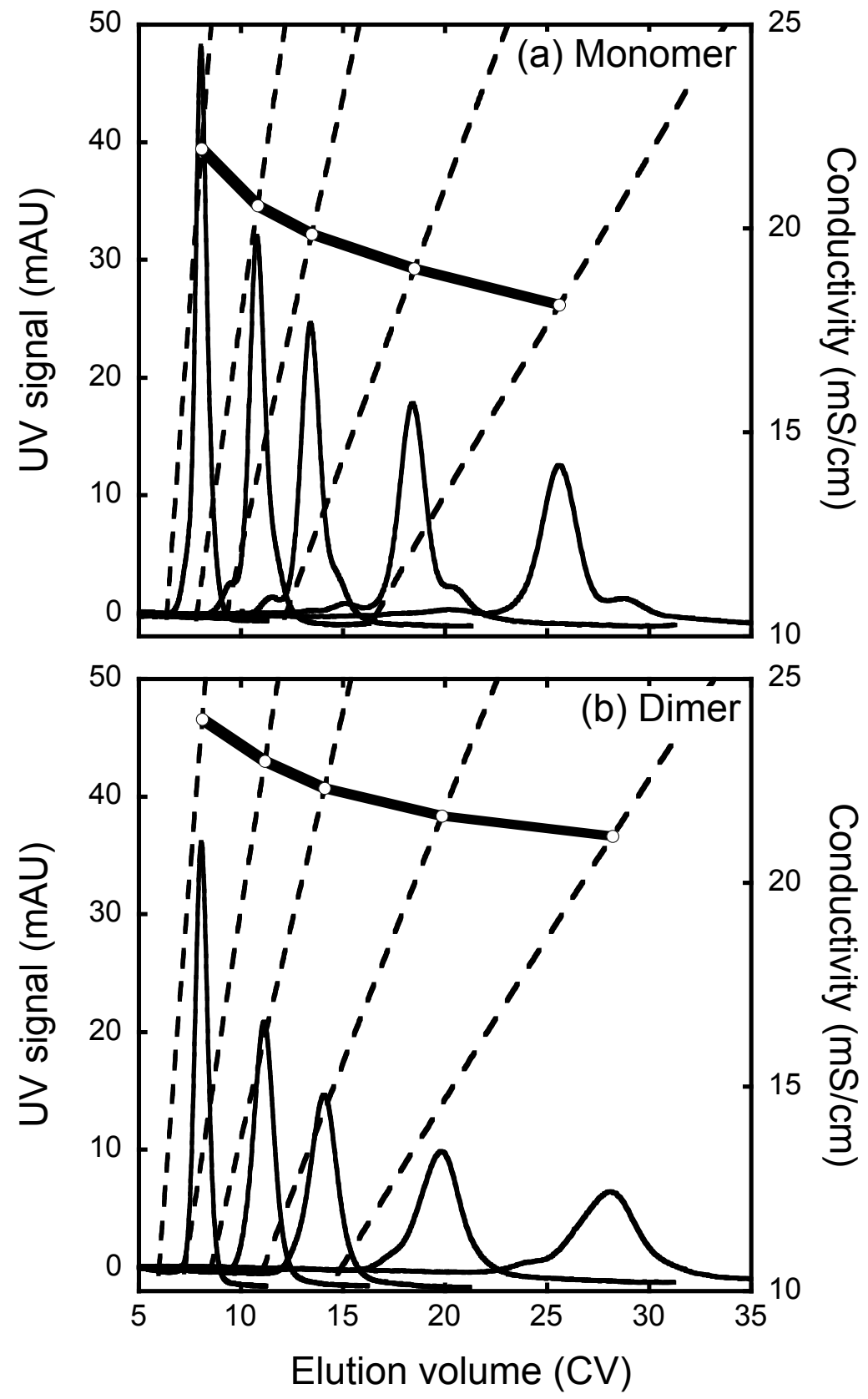

Figure 6.2. LGE chromatograms for (a) monomer and (b) purified dimer with gradients from 0 to $300 \mathrm{mM} \mathrm{NaCl}$ in 5, 10, 15, 25, and $40 \mathrm{CV}$. The dashed lines show the conductivity traces. The thick solid lines connect the conductivity values at which the peak elutes at each gradient slope 


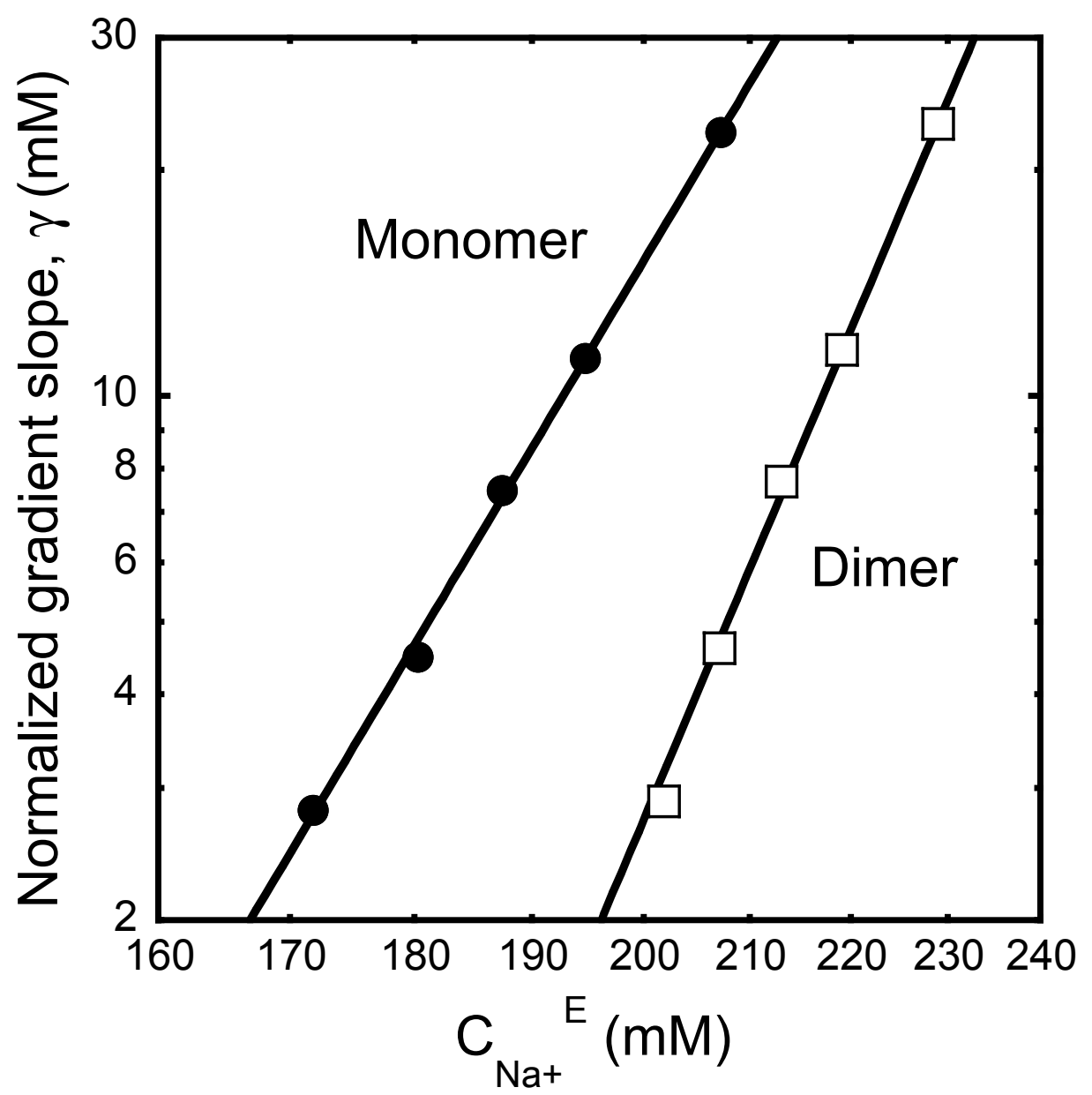

Figure 6.3. Log-log plot of normalized gradient slope, $\gamma$, vs. the $\mathrm{Na}^{+}$concentration at elution, $C_{\mathrm{Na}^{+}}^{E}$, for the LGE experiments of Fig. 6.2. 
$\gamma=\beta L / v$, and the sodium concentration at elution, $C_{\mathrm{Na}^{+}}^{E}$, when $k_{\infty}^{\prime}$ equals the retention factor of salt (Carta et al. 2005):

$$
\log (\gamma)=(z+1) \log \left(C_{\mathrm{Na}^{+}}^{E}\right)-\log [A(z+1)]
$$

Figure 6.3 shows a log-log plot of the LGE data according to this equation. The regressed parameter values are $z=10.2 \pm 0.2$ and $A=(3.23 \pm 2.66) \times 10^{23}$ for the monomer and $z=14.8$ \pm 0.3 and $A=(5.15 \pm 7.66) \times 10^{34}$ for the dimer, with both $A$-values based on $\mathrm{Na}^{+}$ concentration in $\mathrm{mM}$ units. The higher effective charge for the dimer is qualitatively consistent with its larger size, allowing interaction with a greater number of charged ligands on the resin surface. For these dilute conditions, the dimer/monomer selectivity based on Eq. 6.1 is given by:

$\alpha_{D, M}=\frac{A_{D}}{A_{M}}\left(C_{N a^{+}}\right)^{z_{M}-z_{D}}=(1.59 \pm 2.71) \times 10^{11}\left(C_{\mathrm{Na}^{+}}\right)^{-(4.6 \pm 0.4)}$

and is predicted to decrease with increasing $\mathrm{Na}^{+}$concentration. Over the range of salt concentrations where the proteins actually eluted in the experiments $(170-230 \mathrm{mM}), \alpha_{D, M}$ varies between 8.7 and 2.2. Much higher values would be predicted by this equation at lower salt concentrations (e.g. $\alpha_{D, M}=1.7 \times 10^{5}$ at $20 \mathrm{mM} \mathrm{Na}^{+}$), but these large values are likely unreliable, in part because of the large statistical uncertainty of the $A$-values determined from the data fit, and in part because the physical uncertainty of being able to extrapolate beyond the experimental range of salt concentrations.

Figure 6.4 shows the reduced HETP obtained for individual injections of monomer and purified dimer under non-binding conditions (see Section 3.2.4) plotted vs. the term $v^{\prime}\left[k_{\infty}^{\prime} /\left(1+k_{\infty}^{\prime}\right)\right]^{2}$. The term in brackets is included to take into account slight differences in retention caused by somewhat greater exclusion of the dimer from the particle pores. The values of $k_{\infty}^{\prime}$ were 0.768 \pm 0.019 and $0.689 \pm 0.020$ for monomer and dimer, respectively. Both lines in Fig. 6.4 are straight, indicating that band broadening is mass transfer controlled. For these conditions, neglecting external mass transfer, the $c$-term of the van Deemter equation is given by (Carta et al. 2005): 


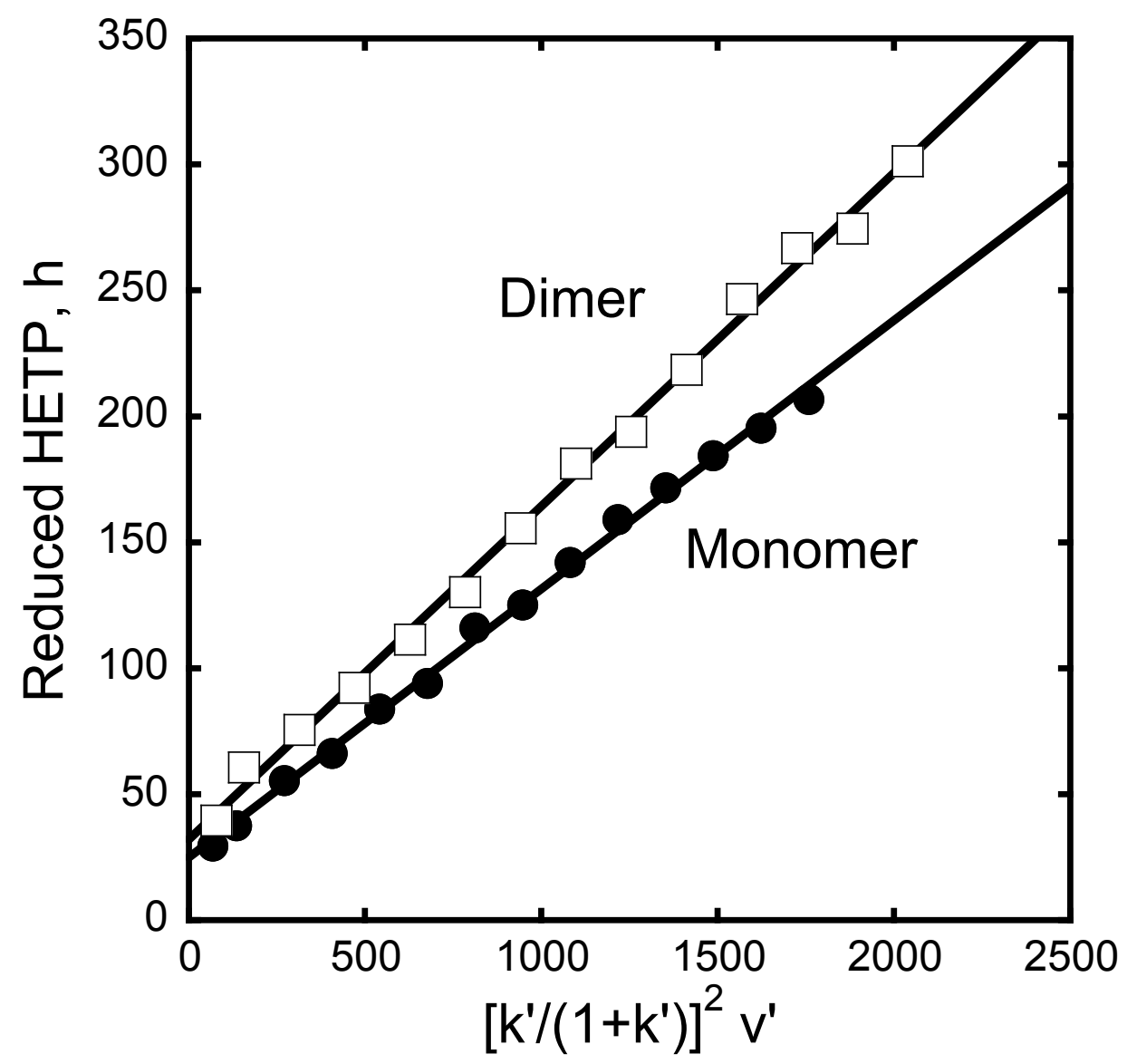

Figure 6.4. Dimensionless van Deemter plot for monomer and dimer obtained from isocratic pulse elution under non-binding conditions. The reduced velocity, $v^{\prime}$, is multiplied times the ratio $\left[k^{\prime} /\left(1+k^{\prime}\right)\right]$ with $k^{\prime}=k_{\infty}^{\prime}$ to account for slight differences in retention 
$c=\frac{1}{30} \frac{\varepsilon}{1-\varepsilon}\left(\frac{k^{\prime}}{1+k^{\prime}}\right)^{2} \frac{D_{0}}{D_{e}}$

where $D_{e}$ is the effective pore diffusivity. The regressed values of $D_{e} / D_{0}$ are $0.195 \pm 0.003$ and $0.157 \pm 0.003$, for monomer and dimer, respectively. The smaller value of this ratio for the dimer is consistent with its larger size, likely resulting in somewhat greater diffusional hindrance. The corresponding effective pore diffusivities are $7.6 \times 10^{-8}$ and $4.7 \times 10^{-8} \mathrm{~cm}^{2} / \mathrm{s}$ for the monomer and dimer, respectively.

\subsection{Adsorption Isotherms}

Figure 6.5 shows the single component adsorption isotherms performed as described in Section 3.2.9 in $10 \mathrm{mM} \mathrm{Na}_{2} \mathrm{HPO}_{4}$ at pH 7.0 with 0,60 , and $100 \mathrm{mM}$ added $\mathrm{NaCl}$. Both the Langmuir isotherm model:

$q=\frac{q_{m} K_{L} C}{1+K_{L} C}$

where $q_{m}$ is the monolayer binding capacity and $K_{L}$ the affinity constant, and the steric mass action (SMA) model (Brooks \& Cramer 1992):

$q=\frac{K_{e}\left[q_{0}-(z+\sigma) q\right]^{z} C}{\left(C_{N a^{+}}\right)^{z}}$

where $K_{e}$ is the equilibrium constant for the exchange of protein and $\mathrm{Na}^{+}$counterions, $q_{0}$ is the resin charge density, $z$ is the protein effective binding charge, and $\sigma$ is the steric hindrance parameter, can be used to fit the data. The regressed values of the parameters appearing in these two equations, $q_{m}$ and $K_{L}$ for the Langmuir model and $q_{0}, K_{e}$, and $\sigma$ for the SMA model are summarized in Table 6.1 and calculated curves are shown in Figure 6.5 by solid and dashed lines, for the monomer and dimer, respectively. In fitting the SMA model parameters, $z$ was assumed to be the same as that determined from the LGE experiments for each species. Although the SMA model fits the data with slightly lower accuracy than the Langmuir model, fewer parameters are needed for the SMA model to fit the entire range of salt concentrations. As seen from the figure and from Table 6.1, at low salt, when binding is 


\begin{tabular}{|c|c|c|c|c|c|c|}
\hline & \multicolumn{6}{|c|}{ Langmuir isotherm parameters } \\
\hline & \multicolumn{2}{|c|}{$0 \mathrm{mM} \mathrm{NaCl}$} & \multicolumn{2}{|c|}{$60 \mathrm{mM} \mathrm{NaCl}$} & \multicolumn{2}{|c|}{$100 \mathrm{mM} \mathrm{NaCl}$} \\
\hline & $\begin{array}{c}q_{m} \\
(\mathrm{mg} / \mathrm{mL})\end{array}$ & $\begin{array}{c}K_{L} \\
\text { (mL/mg) }\end{array}$ & $\begin{array}{c}q_{m} \\
(\mathrm{mg} / \mathrm{mL})\end{array}$ & $\begin{array}{c}K_{L} \\
\text { (mL/mg) }\end{array}$ & $\begin{array}{c}q_{m} \\
(\mathrm{mg} / \mathrm{mL})\end{array}$ & $\begin{array}{c}K_{L} \\
(\mathrm{~mL} / \mathrm{mg})\end{array}$ \\
\hline Monomer & 137 & 145 & 73.3 & 54.4 & 53.1 & 1.17 \\
\hline \multirow[t]{3}{*}{ Dimer } & 145 & 221 & 93.1 & 74.7 & 72.1 & 13.1 \\
\hline & \multicolumn{6}{|c|}{ SMA isotherm parameters } \\
\hline & \multicolumn{2}{|c|}{$q_{0}(\mathrm{mM})$} & $z$ & \multicolumn{2}{|c|}{$K_{e}$} & $\sigma$ \\
\hline Monomer & \multirow{2}{*}{\multicolumn{2}{|c|}{132}} & 10.2 & \multicolumn{2}{|c|}{104} & 111 \\
\hline Dimer & & & 14.8 & \multicolumn{2}{|c|}{$1.33 \times 10^{4}$} & 229 \\
\hline
\end{tabular}

Table 6.1. Langmuir and SMA isotherm parameters obtained by fitting the single-component adsorption data for monomer and dimer in $10 \mathrm{mM} \mathrm{Na}_{2} \mathrm{HPO}_{4}$ at pH 7.0 with the addition of different $\mathrm{NaCl}$ concentrations 

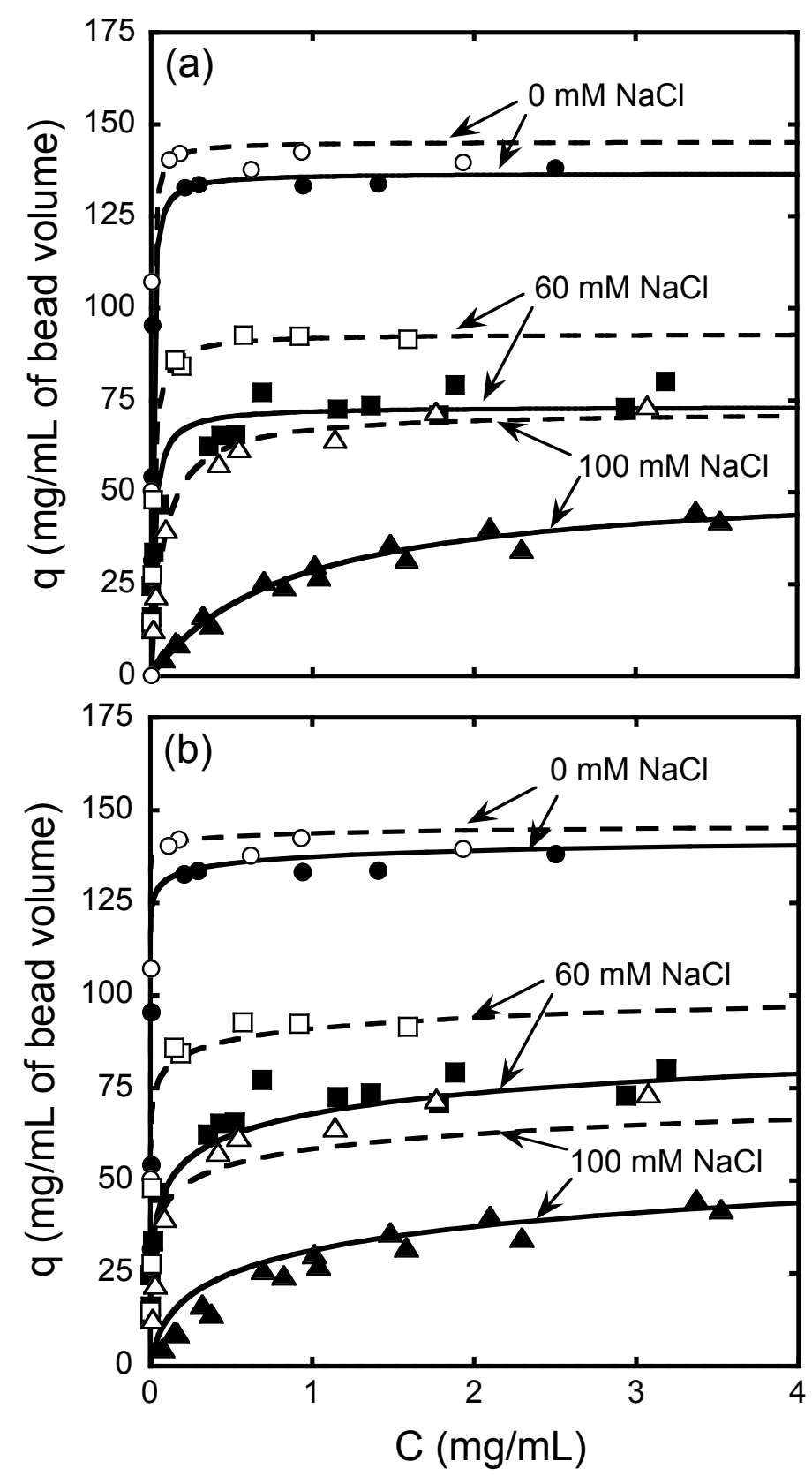

Figure 6.5. Single component adsorption isotherms for mAb monomer (filled symbols) and $m A b$ dimer (open symbols). Solid and dashed lines represent the Langmuir model isotherm in (a) and the SMA model in (b) calculated using the parameters in Table 6.1 
strongest, the binding capacities on a mass basis are nearly the same for monomer and dimer, suggesting that for these conditions the binding orientation is such that the thickness of the bound layer is the same for both species. At higher salt, however, when binding is weaker, preference for the dimer over the monomer becomes more evident as the difference between maximum dimer and monomer capacity increases.

\subsection{Adsorption Kinetics}

Figure 6.6 shows representative confocal laser scanning microscopy (as described in Section 3.2.10) images for single component adsorption of monomer (red) and dimer (green), each with protein concentration of $2.0 \mathrm{mg} / \mathrm{mL}$ in $10 \mathrm{mM} \mathrm{Na}_{2} \mathrm{HPO}_{4}$ at $\mathrm{pH} 7.0$ buffer on similarly sized particles and at comparable times. Both monomer and dimer display a sharp adsorption front. However, the dimer front progresses more slowly despite the fact that, for these conditions, the equilibrium binding capacities are nearly the same. Figure 6.7 shows the dimensionless position of the adsorption fronts, $\rho_{s}=r_{s} / r_{p}$, vs. reduced time, $t / r_{p}^{2}$, where $r_{s}$ is the radial position of the adsorption front and $r_{p}$ is the particle radius. The values of $r_{p}$ and $r_{s}$ were obtained for each image at the inflection points of the digitized, averaged radial fluorescence intensity profiles. The sharp fronts observed experimentally and trends of $\rho_{s}$ vs. time seen in this figure are consistent with a pore diffusion mechanism. Accordingly, since the isotherm is highly favorable for these conditions, the effective pore diffusivity was determined by comparing the data in Fig. 6.7 with the following equation, which is based on the well-known shrinking core model (Ruthven 1983; Carta \& Jungbauer 2010):

$$
2 \rho_{s}^{3}-3 \rho_{s}^{2}+1=\frac{6 D_{0} C t}{q_{m} r_{p}^{2}} \frac{D_{e}}{D_{0}}
$$

where $C$ is the protein solution concentration and $q_{m}$ is the protein binding capacity. The fitted values of $D_{e} / D_{0}$ are $0.204 \pm 0.006$ and $0.137 \pm 0.002$ for monomer and dimer, respectively. These ratios, corresponding to $D_{e}$-values of $8.0 \times 10^{-8}$ and $4.1 \times 10^{-8} \mathrm{~cm}^{2} / \mathrm{s}$ for the monomer and dimer, respectively, are in fairly good agreement with the values obtained under 


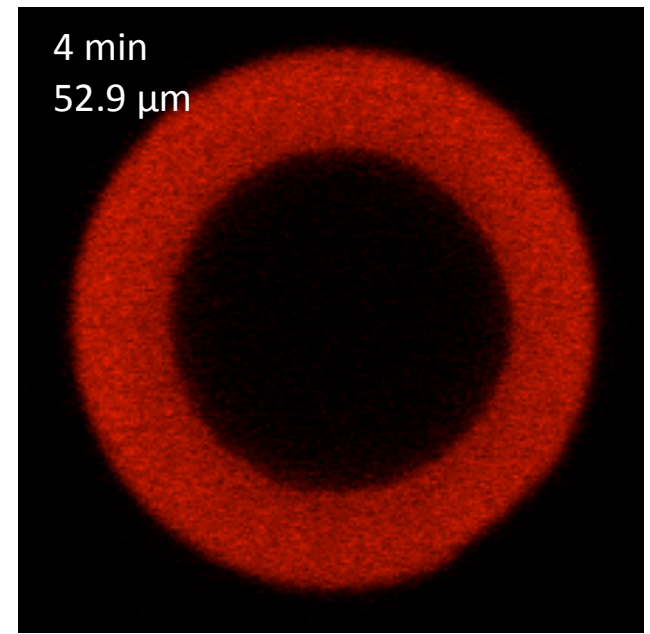

\section{$4 \mathrm{~min}$ \\ $52.9 \mu \mathrm{m}$}

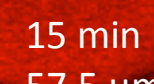

$57.5 \mu \mathrm{m}$

$16 \mathrm{~min}$

$55.7 \mu \mathrm{m}$
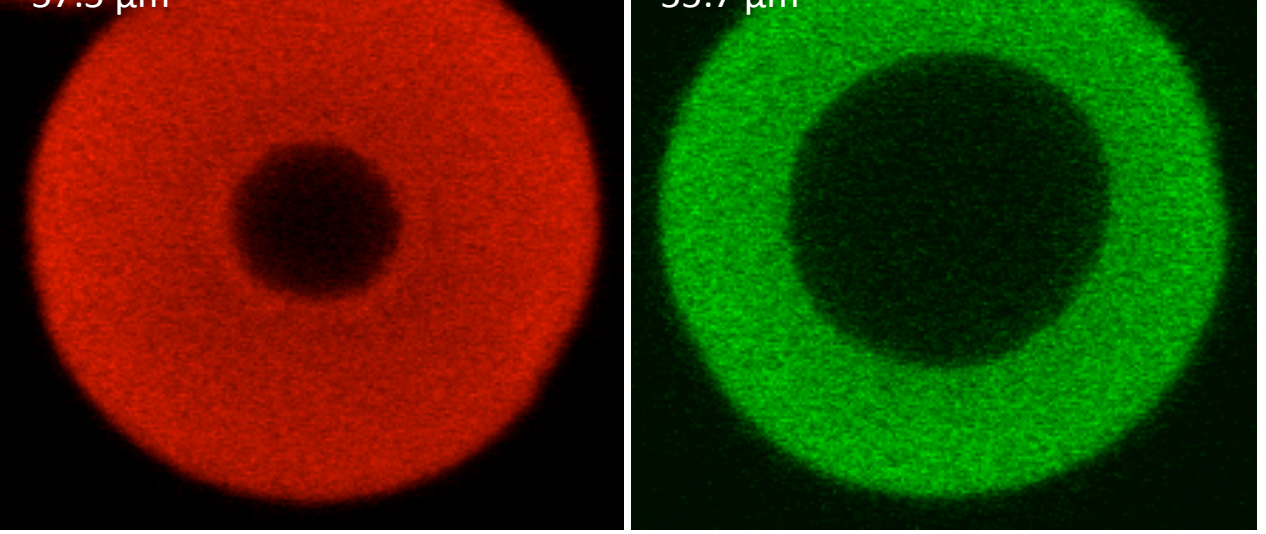

Figure 6.6. Representative CLSM images of single component adsorption of mAb monomer (left, red) and mAb dimer (right, green) on similarly sized resin particles at comparable times from $2.0 \mathrm{mg} / \mathrm{mL}$ solutions in $10 \mathrm{mM} \mathrm{Na}_{2} \mathrm{HPO}_{4}$ at $\mathrm{pH}$ 7.0. Actual times and particle diameters are shown in the insets 


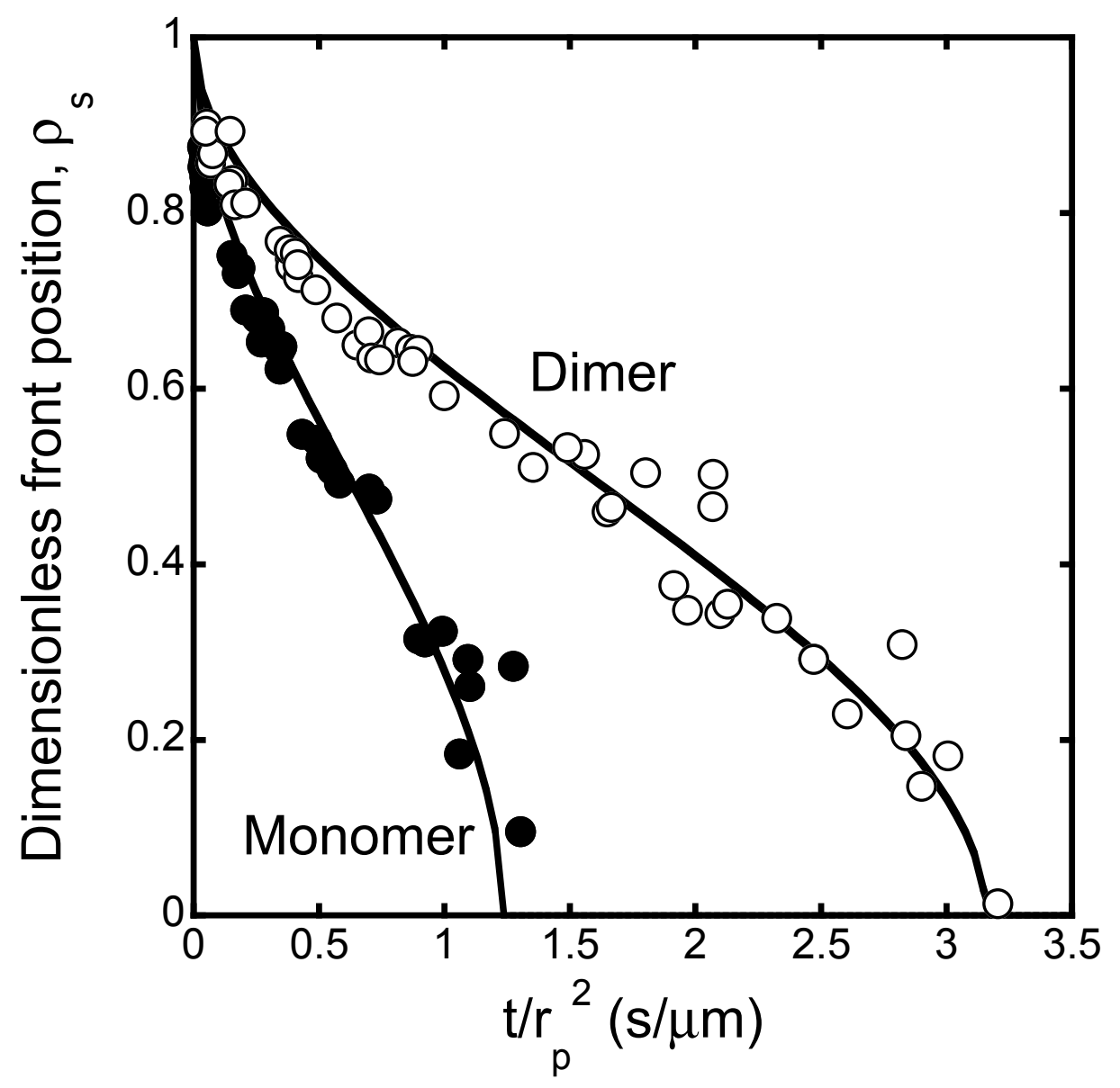

Figure 6.7. Dimensionless front positions vs. reduced time for single component adsorption of mAb monomer and dimer for the conditions of Fig. 6.6. Lines are based on Eq. 6.7 with $D_{e} / D_{0}=0.204$ and 0.137 for monomer and dimer, respectively 
non-binding conditions for the same species ( 0.195 and 0.157 , respectively), suggesting that the single component adsorption kinetics remains limited by pore diffusion for both species with no evidence of a significant kinetic resistance to binding.

As noted by previous authors (e.g. Carta et al. 2005; Teske et al. 2005; Teske et al. 2006; Teske et al. 2007), a potential limitation of confocal microscopy using fluorescent protein conjugates is that labeling may alter the protein charge and hydrophobicity, thereby affecting interactions with the resin and resulting in artifacts such as the appearance of concentration overshoots due to competitive binding between labeled and unlabeled protein molecules. In order to determine whether this phenomenon affected our results, linear gradient elution experiments were performed with labeled and unlabeled mAb monomer and dimer at $\mathrm{pH} 7.0$ on a Nuvia HR$S$ column with detection at $280 \mathrm{~nm}$ for the unlabeled protein and at 570 and $503 \mathrm{~nm}$ for the labeled monomer and dimer, respectively, which are the UV/Vis absorption maxima of the red and green dyes used. As shown in Fig. 6.8, there was no detectable difference in either retention or peak shape between the unlabeled and labeled species indicating that in our case, labeling did not significantly affect interactions with the resin. 

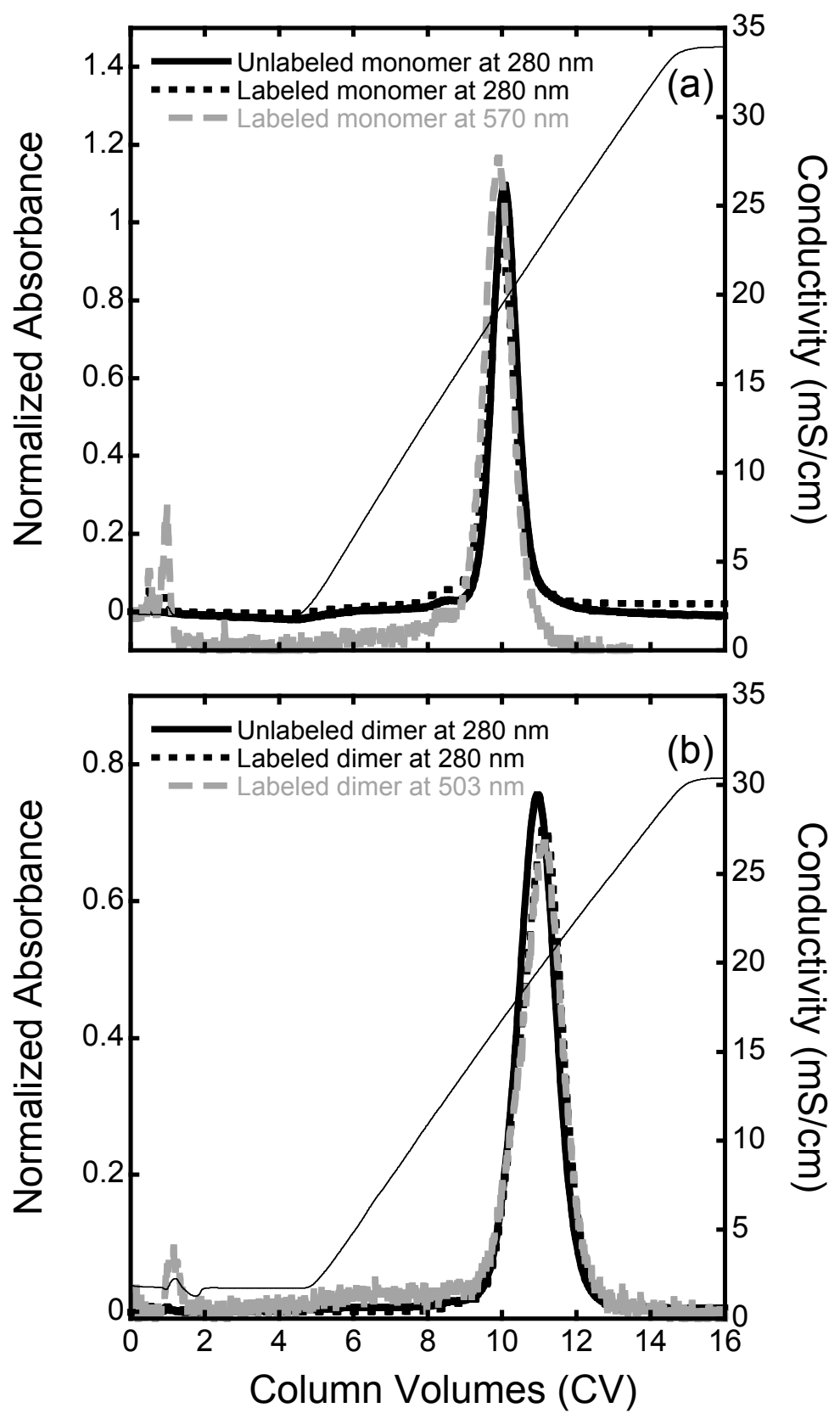

Figure 6.8. LGE chromatograms of labeled and unlabeled (a) monomer and (b) dimer feedstocks on Nuvia HR-S. $10 \mathrm{CV}$ gradient from $10 \mathrm{mM} \mathrm{Na}_{2} \mathrm{HPO}_{4}, \mathrm{pH} 7.0$ to $10 \mathrm{mM} \mathrm{Na}{ }_{2} \mathrm{HPO}_{4}, 300 \mathrm{mM} \mathrm{NaCl}$, $\mathrm{pH} 7.0$ 


\section{Characterization of Two-Component Adsorption Equilibrium and Kinetics of Antibody Monomer and Dimer on Nuvia HR-S}

The previous chapter characterized the behavior of the mAb monomer and dimer individually using a variety of experimental techniques. This chapter extends two of those techniques, adsorption isotherms and confocal laser scanning microscopy, to examine the two-component response of the monomer and dimer within the framework of the previously described equilibrium and kinetics models.

\subsection{Adsorption Isotherms}

Two-component isotherms of mAb monomer and dimer were evaluated at varying salt concentrations as described in Section 3.2.9. In this instance, analytical SEC was used to evaluate the percentage of monomer and dimer in the feed and supernatant after equilibration in order to complete the material balances. Table 7.1 shows the two-component adsorption equilibrium data in $10 \mathrm{mM} \mathrm{Na}_{2} \mathrm{HPO}_{4}$ at pH 7.0 with $0,10,30$, and $60 \mathrm{mM}$ added $\mathrm{NaCl}$ and various ratios of monomer and dimer concentrations, $C_{M}$ and $C_{D}$, respectively, in the supernatant at equilibrium. Both solution and bound concentrations are shown in mass concentration units $(\mathrm{mg} / \mathrm{mL})$ and the selectivity is calculated as:

$\alpha_{D, M}=\frac{q_{D}}{C_{D}} \frac{C_{M}}{q_{M}}$

As seen from this table, the selectivity varies with both salt concentration and with the relative concentration of monomer and dimer in the equilibrated supernatant. In general, the selectivity is low at low salt, when protein binding is strong and the total protein bound is greatest, averaging around 1.2 and, in some cases, even becoming smaller than 1 with no $\mathrm{NaCl}$ added. The selectivity increases steadily with salt concentration, as binding becomes weaker and the total bound protein concentration decreases, eventually reaching values averaging around 8.4 with $60 \mathrm{mM}$ added $\mathrm{NaCl}$. Consistent trends of increasing selectivity are also seen at each salt concentration as the ratio of monomer to dimer in the equilibrated supernatant increases. 


\begin{tabular}{|c|c|c|c|c|c|c|}
\hline $\begin{array}{l}C_{\mathrm{Na}^{+}} \\
(\mathrm{mM})\end{array}$ & $\begin{array}{c}C_{M} \\
(\mathrm{mg} / \mathrm{mL})\end{array}$ & $\begin{array}{c}C_{D} \\
(\mathrm{mg} / \mathrm{mL})\end{array}$ & $\begin{array}{c}q_{\text {total }} \\
(\mathrm{mg} / \mathrm{mL})\end{array}$ & $\begin{array}{c}q_{M} \\
(\mathrm{mg} / \mathrm{mL})\end{array}$ & $\begin{array}{c}q_{D} \\
(\mathrm{mg} / \mathrm{mL})\end{array}$ & $\alpha_{D, M}$ \\
\hline \multirow{9}{*}{0} & 0.56 & 0.53 & 159.2 & 79.7 & 79.5 & 1.05 \\
\hline & 0.71 & 1.26 & 167.3 & 66.9 & 100.4 & 0.85 \\
\hline & 0.81 & 2.09 & 161.6 & 57.9 & 103.7 & 0.69 \\
\hline & 1.27 & 0.55 & 164.8 & 81 & 83.8 & 2.39 \\
\hline & 1.47 & 1.41 & 146.8 & 63.8 & 83 & 1.36 \\
\hline & 1.58 & 2.31 & 143.1 & 69.7 & 73.4 & 0.72 \\
\hline & 2.12 & 0.78 & 147.6 & 84.2 & 63.4 & 2.05 \\
\hline & 2.25 & 1.58 & 149.9 & 83.1 & 66.8 & 1.14 \\
\hline & 2.25 & 2.47 & 161.9 & 90.5 & 71.4 & 0.72 \\
\hline \multirow{4}{*}{10} & 0.58 & 0.3 & 128.2 & 56.6 & 71.6 & 2.5 \\
\hline & 0.65 & 1.03 & 127.2 & 53.8 & 73.4 & 0.85 \\
\hline & 1.31 & 0.3 & 126.5 & 65.2 & 61.3 & 4.14 \\
\hline & 1.48 & 1.18 & 126.3 & 59.7 & 66.6 & 1.4 \\
\hline \multirow{4}{*}{30} & 0.7 & 0.14 & 110.5 & 33.4 & 77.1 & 11.8 \\
\hline & 0.79 & 0.75 & 108.1 & 24.5 & 83.6 & 3.58 \\
\hline & 1.52 & 0.18 & 107.5 & 39.3 & 68.2 & 14.5 \\
\hline & 1.58 & 0.8 & 112.7 & 35.9 & 76.8 & 4.24 \\
\hline \multirow{6}{*}{60} & 0.34 & 0.15 & 82.9 & 12.4 & 70.5 & 12.9 \\
\hline & 0.48 & 0.11 & 91.2 & 21.9 & 69.3 & 13.6 \\
\hline & 0.43 & 0.73 & 89.5 & 11.9 & 77.6 & 3.86 \\
\hline & 0.6 & 0.88 & 105 & 28.4 & 76.6 & 1.83 \\
\hline & 1.05 & 0.18 & 92.6 & 25.4 & 67.2 & 15.8 \\
\hline & 1.26 & 1.01 & 98.4 & 20.6 & 77.8 & 4.73 \\
\hline
\end{tabular}

Table 7.1. Two-component adsorption equilibrium data for $\mathrm{mAb}$ monomer/dimer mixtures in $10 \mathrm{mM} \mathrm{Na}_{2} \mathrm{HPO}_{4}$ at pH 7.0 with the addition of different $\mathrm{NaCl}$ concentrations 

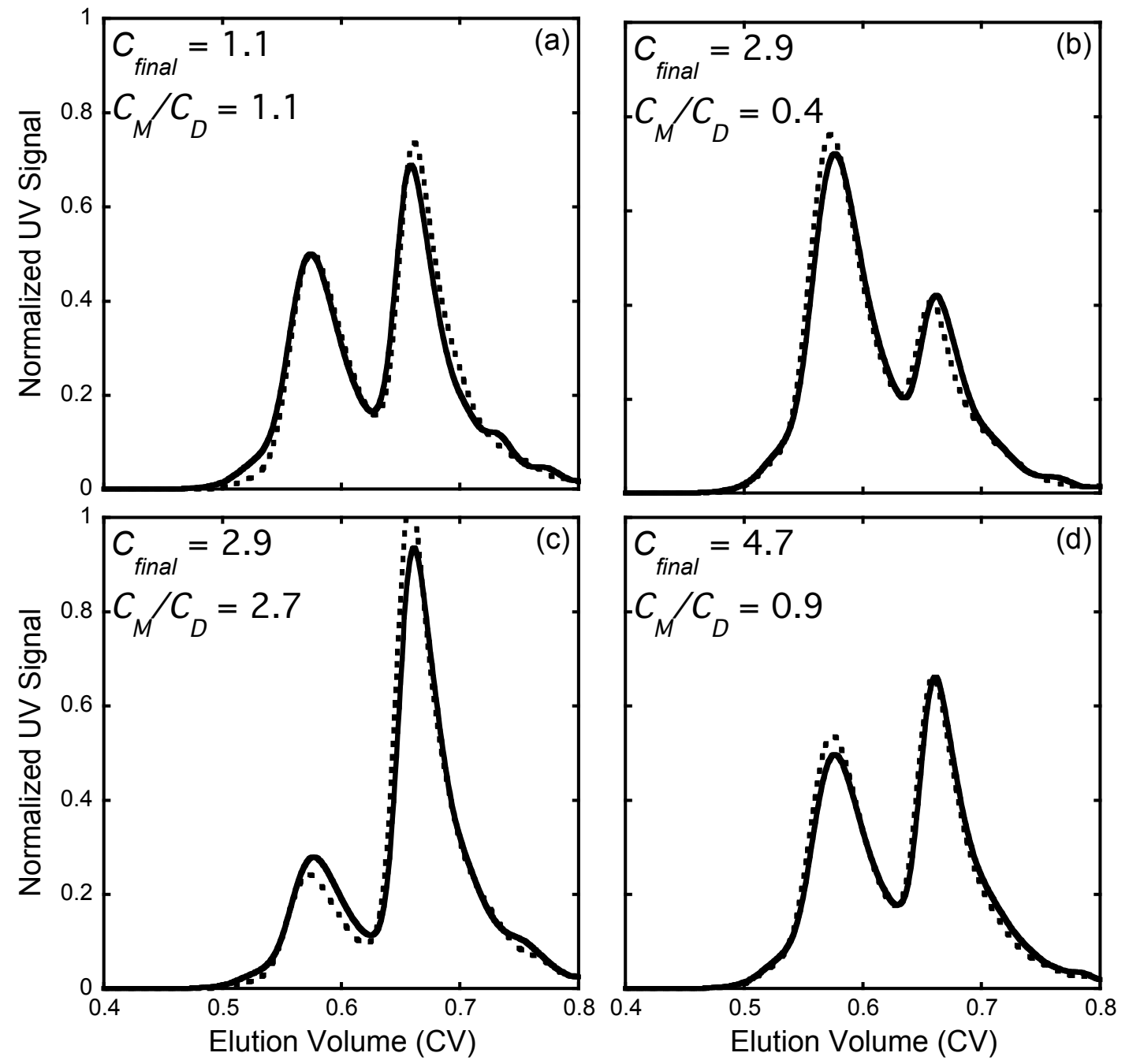

Figure 7.1. SEC chromatograms of two-component isotherm feed and supernatant for a mAb monomer and dimer mixture at $20 \mathrm{mM} \mathrm{Na}^{+}$at varying initial ratios of monomer and dimer. Solid line is the isotherm feed before contact with resin, dashed line is isotherm supernatant at equilibrium. Inset text shows final total protein concentration $(\mathrm{mg} / \mathrm{mL})$ and ratio of monomer to dimer mass concentration 

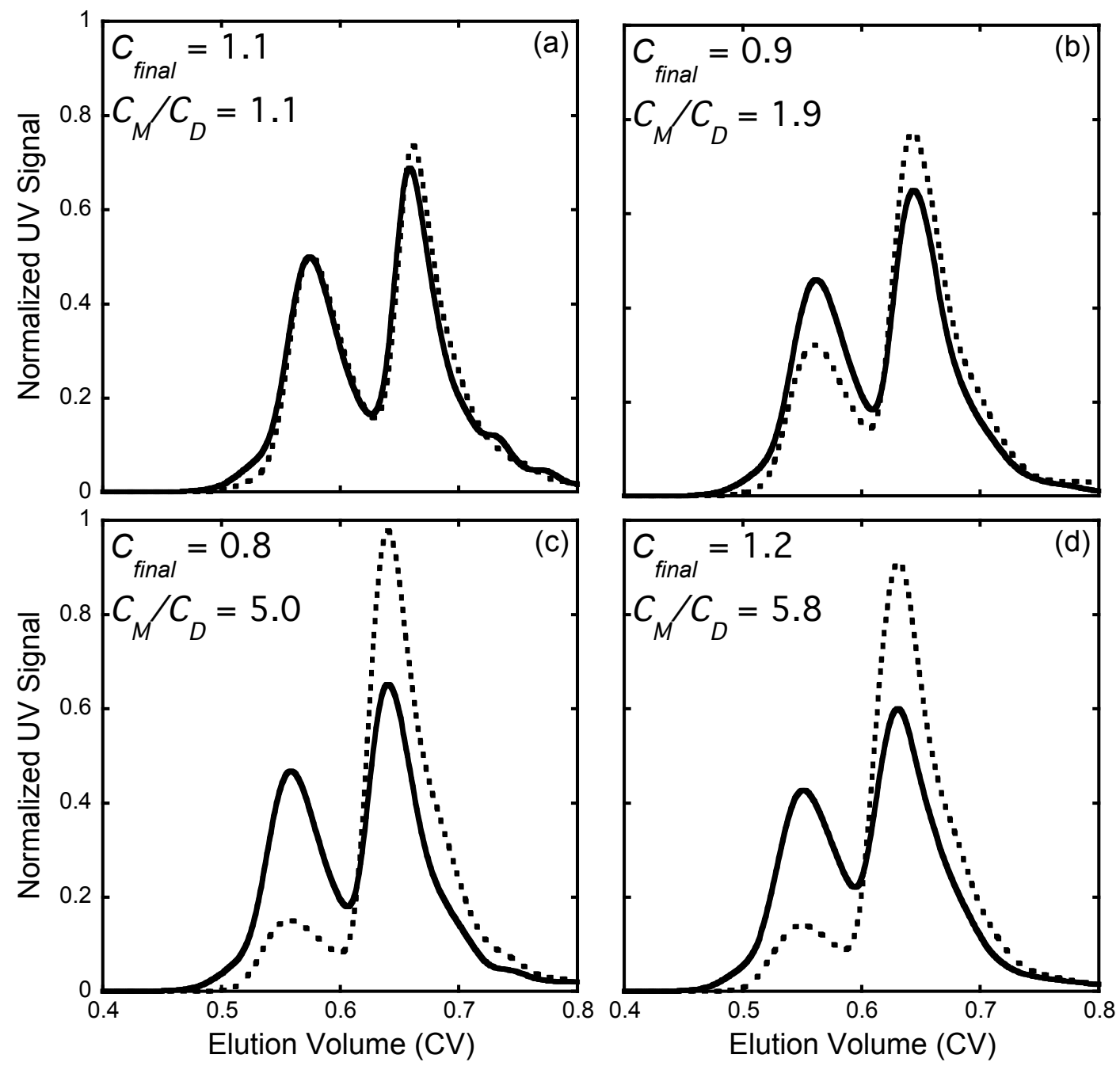

Figure 7.2. SEC chromatograms of two-component isotherm feed and supernatant for a mAb monomer and dimer mixture at (a) $20 \mathrm{mM}$, (b) $30 \mathrm{mM}$, (c) $50 \mathrm{mM}$, and (d) $80 \mathrm{mM} \mathrm{Na}^{+}$. Solid line is the isotherm feed before contact with resin, dashed line is isotherm supernatant at equilibrium. Inset text shows final total protein concentration $(\mathrm{mg} / \mathrm{mL})$ and ratio of monomer to dimer mass concentration 
Figures 7.1 and 7.2 show the SEC chromatograms of a subset of these experiments, with Fig. 7.1 showing the $20 \mathrm{mM} \mathrm{Na}^{+}$experiments that display little selectivity, and Fig. 7.2 showing a similar feed composition with gradually increasing $\mathrm{Na}^{+}$that shows the gradual depletion of the dimer peak in the eluate, owing to increased selectivity.

Unfortunately, neither the multicomponent version of the Langmuir model, nor the multicomponent version of the SMA model, is consistent with these trends. According to the multicomponent Langmuir, the selectivity at each salt concentration is given by (Ruthven 1983; Carta \& Jungbauer 2010):

$\alpha_{D, M}=\frac{q_{m, D} K_{L, D}}{q_{m, M} K_{L, M}}$

which gives values of $\alpha_{D, M}=1.6$ with no salt added and 1.7 with $60 \mathrm{mM}$, independent of the monomer-to-dimer concentration ratio, and quite different from the corresponding experimental average values of 1.2 and 8.4. According to the multicomponent SMA model, the selectivity is given by (Lewus \& Carta 1999):

$\alpha_{D, M}=\frac{K_{e, D}}{K_{e, M}}\left[q_{0}-\left(z_{M}+\sigma_{M}\right) q_{M}-\left(z_{D}+\sigma_{D}\right) q_{D}\right]^{z_{D}-z_{M}}\left(C_{N a^{+}}\right)^{z_{M}-z_{D}}$

This equation predicts a protein concentration dependence qualitatively consistent with the experimental data, i.e. $\alpha_{D, M}$ increasing as the monomer-to-dimer ratio in the equilibrated supernatant increases, but predicts a trend with regards to the effects of salt concentration inconsistent with the experimental data, with $\alpha_{D, M}$ decreasing rather than increasing as seen experimentally when the salt concentration is increased.

The specific reasons why neither the Langmuir model nor the SMA could describe quantitatively the experimental selectivities at high protein loads are not precisely known. A possibility is that this is due to the vastly different sizes of the monomer and dimer, which are not accounted for explicitly in either isotherm model. The two-component isotherm models of Gu et al. (1991) and Garke et al. (1999) that take into account the different sizes of the bound proteins by assuming an uneven surface saturation were also tested against the data, but also failed to 
predict quantitatively the selectivities observed experimentally. Thus, it seems that a new model is needed that takes into account both ion exchange and mutual size exclusion effects on the adsorbent surface.

\subsection{Adsorption Kinetics}

Figure 7.3 shows confocal laser scanning microscopy (CLSM) images for the adsorption of a mixture containing approximately equal concentrations of monomer and dimer (1.15 and 1.02 $\mathrm{mg} / \mathrm{mL}$, respectively) in $10 \mathrm{mM} \mathrm{Na}_{2} \mathrm{HPO}_{4}$ at $\mathrm{pH} 7.0$ (see Section 3.2.10). For these conditions, a single adsorption front is seen despite the fact that the monomer and dimer have different effective diffusivities. A slight overshoot is also seen for the dimer near the center of the bead at intermediate times. This slight overshoot is attributed to the presence of a small amount of monomer present in the dimer sample. As shown in Fig. 6.8, labeled and unlabeled species exhibited the same chromatographic retention behavior, suggesting that the labeled species are representative of the behavior of the bulk of the protein in both monomer or dimer samples. At long times, the resin is obviously saturated with a mixture of monomer and dimer consistent with the low selectivity obtained from the equilibrium measurements (Table 7.1). If the results in Fig. 7.3 are treated assuming that there is no selectivity between monomer and dimer, the single combined front seen in the images yields a plot of front position vs. reduced time, $t / r_{p}^{2}$, shown in Fig. 7.4. Regressing the data in this plot according to Eq. 6.7, with $q_{m}=$ $141 \mathrm{mg} / \mathrm{mL}$, which is the average of the monomer and dimer binding capacities for these conditions, and $C=2.17 \mathrm{mg} / \mathrm{mL}$, which is the total protein solution concentration used in this experiment, yields $D_{e}=5.2 \times 10^{-8} \mathrm{~cm}^{2} / \mathrm{s}$. Interestingly, this value is intermediate between those of the monomer and the dimer, suggesting that the combined front moves at a rate intermediate between those observed in the corresponding single component experiments. In turn, this result suggests that for these strong binding conditions, the diffusion fluxes of monomer and dimer are coupled, which can occur if, for example, the kinetics of exchange of the two bound species is slow. 
Figure 7.5 shows the results of a sequential adsorption experiment also in $10 \mathrm{mM} \mathrm{Na}_{2} \mathrm{HPO}_{4}$ at $\mathrm{pH}$ 7.0. In this case, the resin was first allowed to equilibrate with an excess amount of approximately $2 \mathrm{mg} / \mathrm{mL} \mathrm{mAb}$ monomer for several hours and then washed with the load buffer. Following removal of the supernatant with a microcentrifuge filter, the resin was then added to a large volume of $1.91 \mathrm{mg} / \mathrm{mL}$ dimer in the same buffer and CLSM images were then collected as described in Section 3.2.10. As seen in Fig. 7.5, gradual replacement of the pre-adsorbed monomer by the dimer occurs, but proceeds only slowly and even after 120 minutes, a significant amount of monomer is still bound to the resin.

Figure 7.6 shows the results for the adsorption of a mixture containing approximately the same concentrations of monomer and $\operatorname{dimer}(1.15 \mathrm{mg} / \mathrm{mL}$ and $0.96 \mathrm{mg} / \mathrm{mL}$, respectively) in $10 \mathrm{mM}$ $\mathrm{Na}_{2} \mathrm{HPO}_{4}$ at $\mathrm{pH} 7.0$, but with the addition of $10 \mathrm{mM} \mathrm{NaCl}$. In this case, a pure monomer front is observed moving toward the center of the particle, closely followed by a mixed front containing both monomer and dimer. Consistent with the higher selectivity obtained from the equilibrium measurements at this salt concentration (Table 7.1), partial displacement of the monomer by the dimer occurs, resulting in an inner band of concentrated monomer that moves toward the center of the particle ahead of the mixed monomer/dimer front. A ring of green fluorescence is also seen at the intermediate time at the same location as the displaced monomer front. As in the case of Fig. 7.3, we believe that this is a result of either residual monomer in the purified dimer sample or a labeling artifact.

Figure 7.7 shows the results for the adsorption of a mixture containing similar concentrations of monomer and dimer (1.16 and $0.84 \mathrm{mg} / \mathrm{mL}$, respectively) also in $10 \mathrm{mM} \mathrm{Na}{ }_{2} \mathrm{HPO}_{4}$ at pH 7.0, but now with that addition of $60 \mathrm{mM} \mathrm{NaCl}$. A clear separation of the two components within the particle is seen at this higher salt concentration, indicating substantial displacement of the monomer by the dimer. In this case, competition between the slower diffusing but more strongly held dimer and the faster diffusing but less strongly bound monomer results in the formation of two adsorption fronts - a faster moving front separating a monomer-saturated zone from a protein-free shrinking core, and a slower moving front, separating the monomer- 

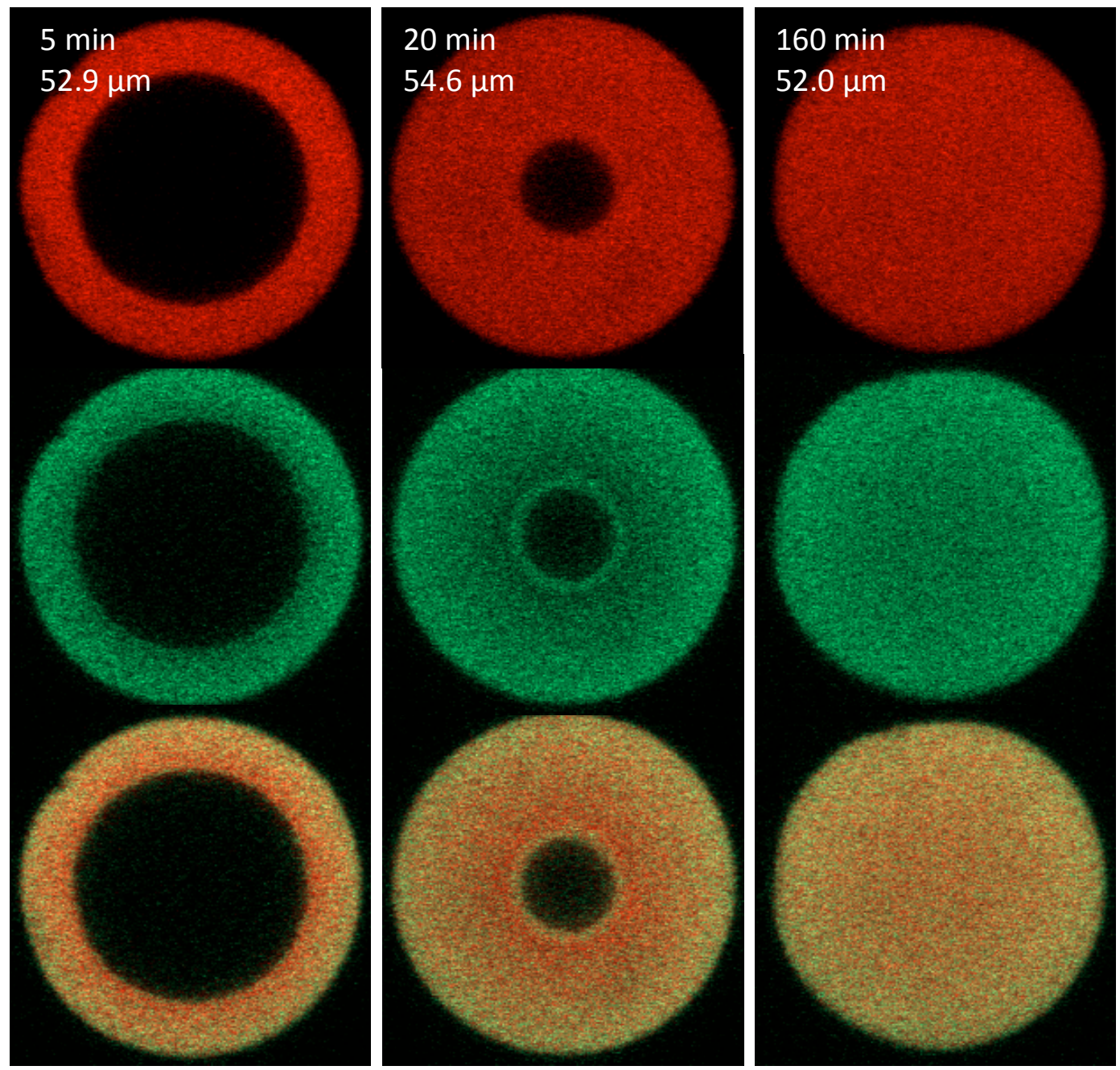

Figure 7.3. Representative CLSM images of two-component adsorption of a mixture containing $1.15 \mathrm{mg} / \mathrm{mL} \mathrm{mAb}$ monomer and $1.02 \mathrm{mg} / \mathrm{mL} \mathrm{mAb}$ dimer in $10 \mathrm{mM} \mathrm{Na}{ }_{2} \mathrm{HPO}_{4}$ at $\mathrm{pH}$ 7.0. The top row (red) shows the monomer profile, the middle row (green) the dimer profile, and the bottom row a digital superposition of the monomer and dimer signals. Actual times and particle diameters are shown in the insets 


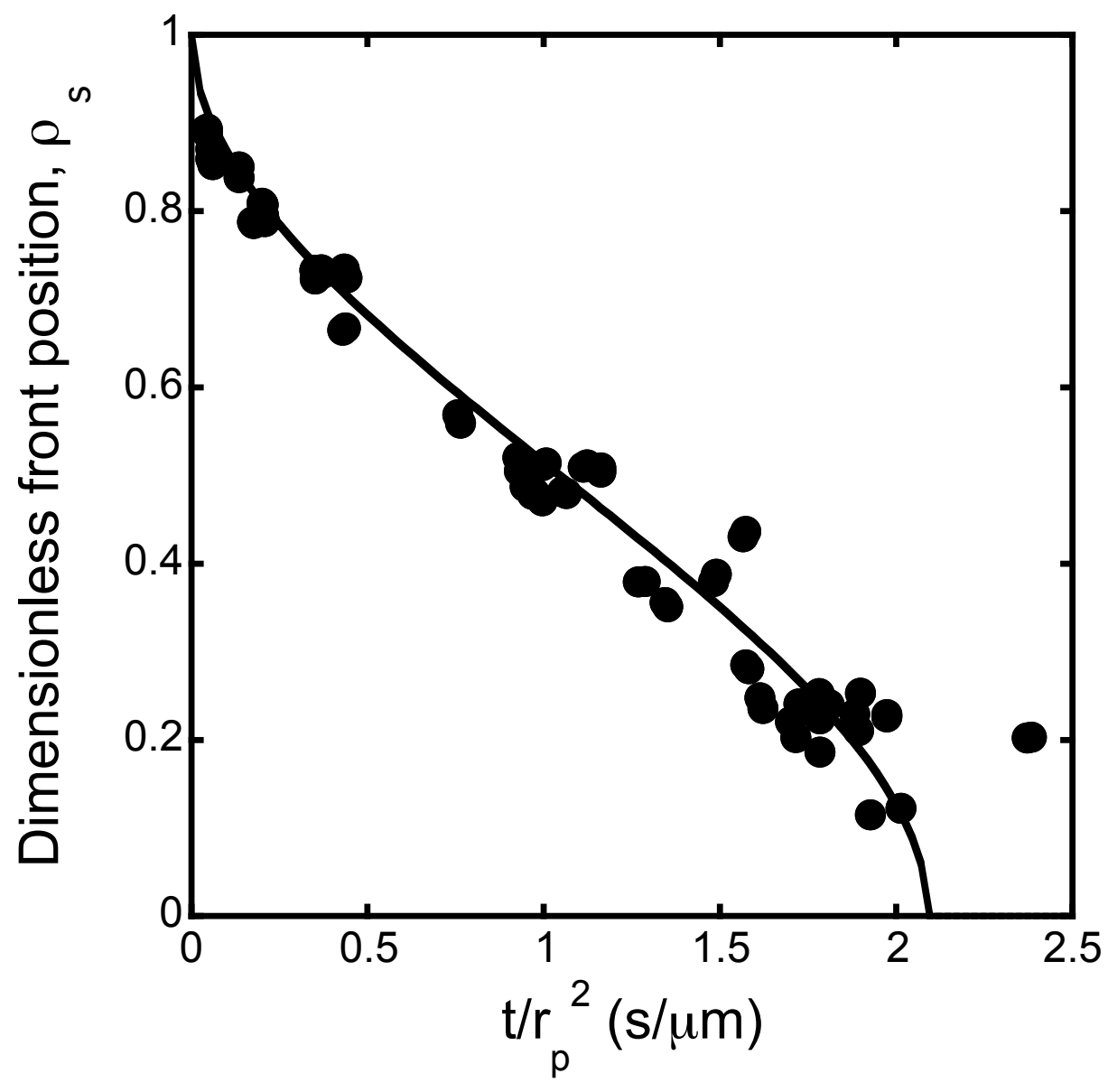

Figure 7.4. Dimensionless front position vs. reduced time for pseudo one-component adsorption of mAb monomer and dimer for the conditions of Fig. 7.3. Line is based on Eq. 6.7 with averaged values of $q_{m}$ and $C$ for monomer and dimer 

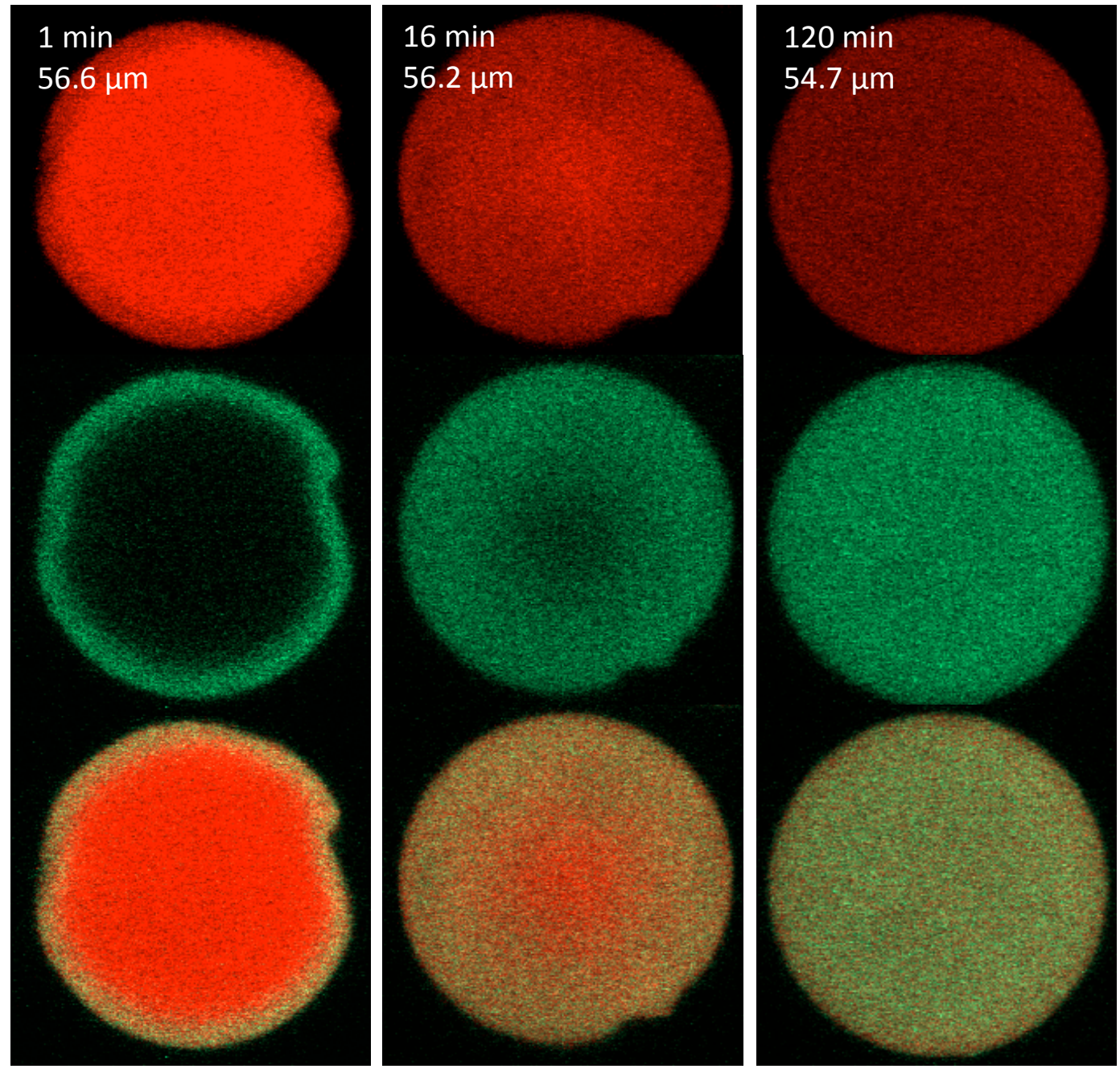

Figure 7.5. Representative CLSM images of adsorption of $1.91 \mathrm{mg} / \mathrm{mL} \mathrm{mAb}$ dimer on particles initially saturated with approximately $2 \mathrm{mg} / \mathrm{mL} \mathrm{mAb}$ monomer in $10 \mathrm{mM} \mathrm{Na} \mathrm{HPO}_{4}$ at pH 7.0. The top row (red) shows the monomer profile, the middle row (green) the dimer profile, and the bottom row a digital superposition of the monomer and dimer signals. Actual times and particle diameters are shown in the insets 

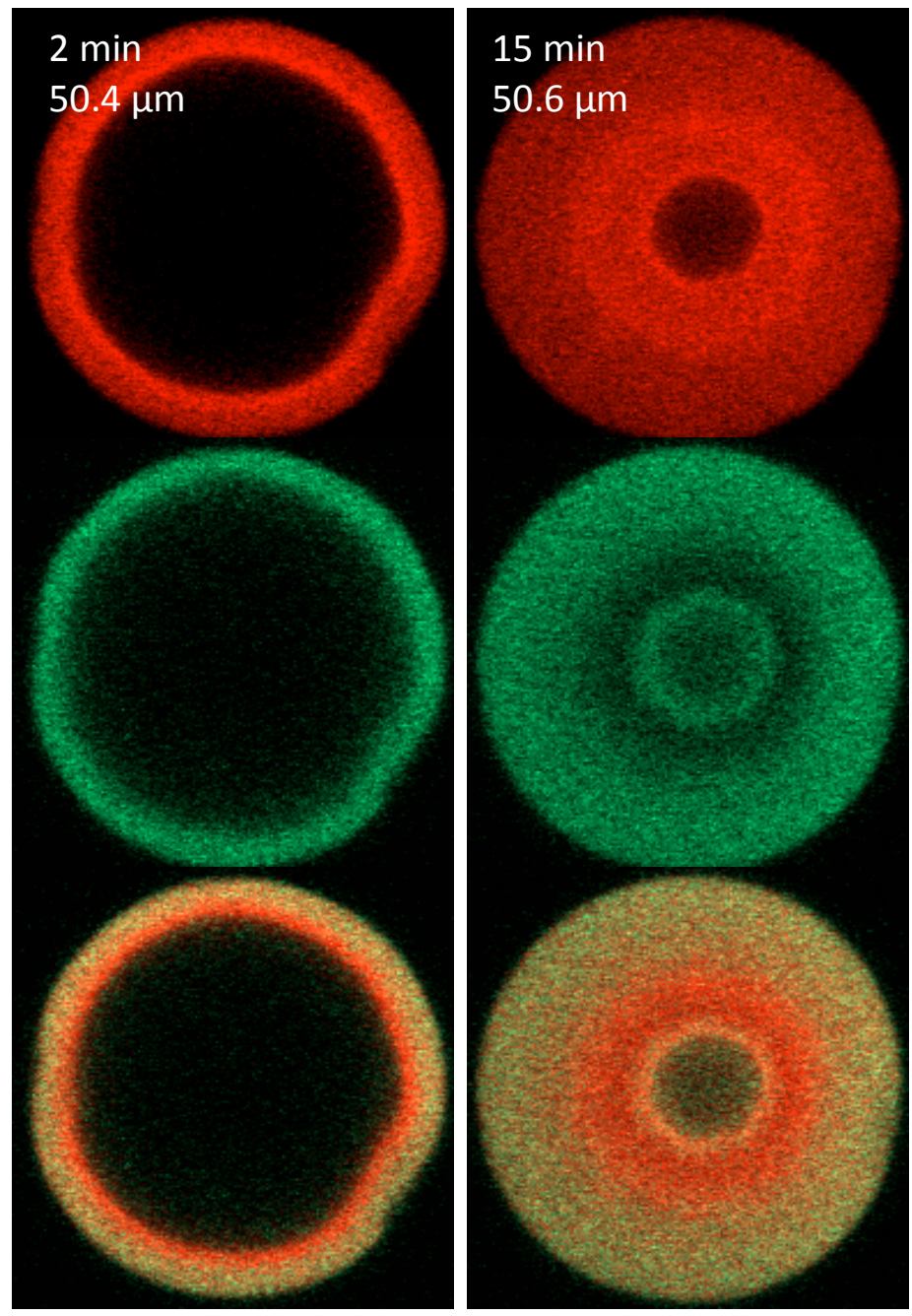

\section{$50 \mathrm{~min}$ \\ $50.6 \mu \mathrm{m}$}

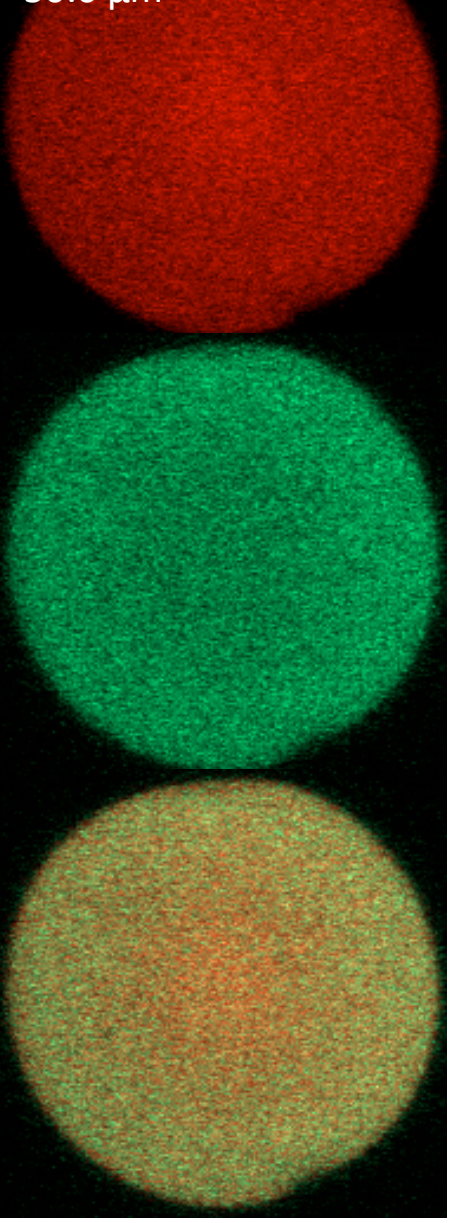

Figure 7.6. Representative CLSM images of two-component adsorption of a mixture containing $1.15 \mathrm{mg} / \mathrm{mL} \mathrm{mAb}$ monomer and $0.96 \mathrm{mg} / \mathrm{mL} \mathrm{mAb}$ dimer in $10 \mathrm{mM} \mathrm{Na}{ }_{2} \mathrm{HPO}_{4}$ at $\mathrm{pH} 7.0$ with 10 $\mathrm{mM}$ added $\mathrm{NaCl}$. The top row (red) shows the monomer profile, the middle row (green) the dimer profile, and the bottom row a digital superposition of the monomer and dimer signals. Actual times and particle diameters are shown in the insets 

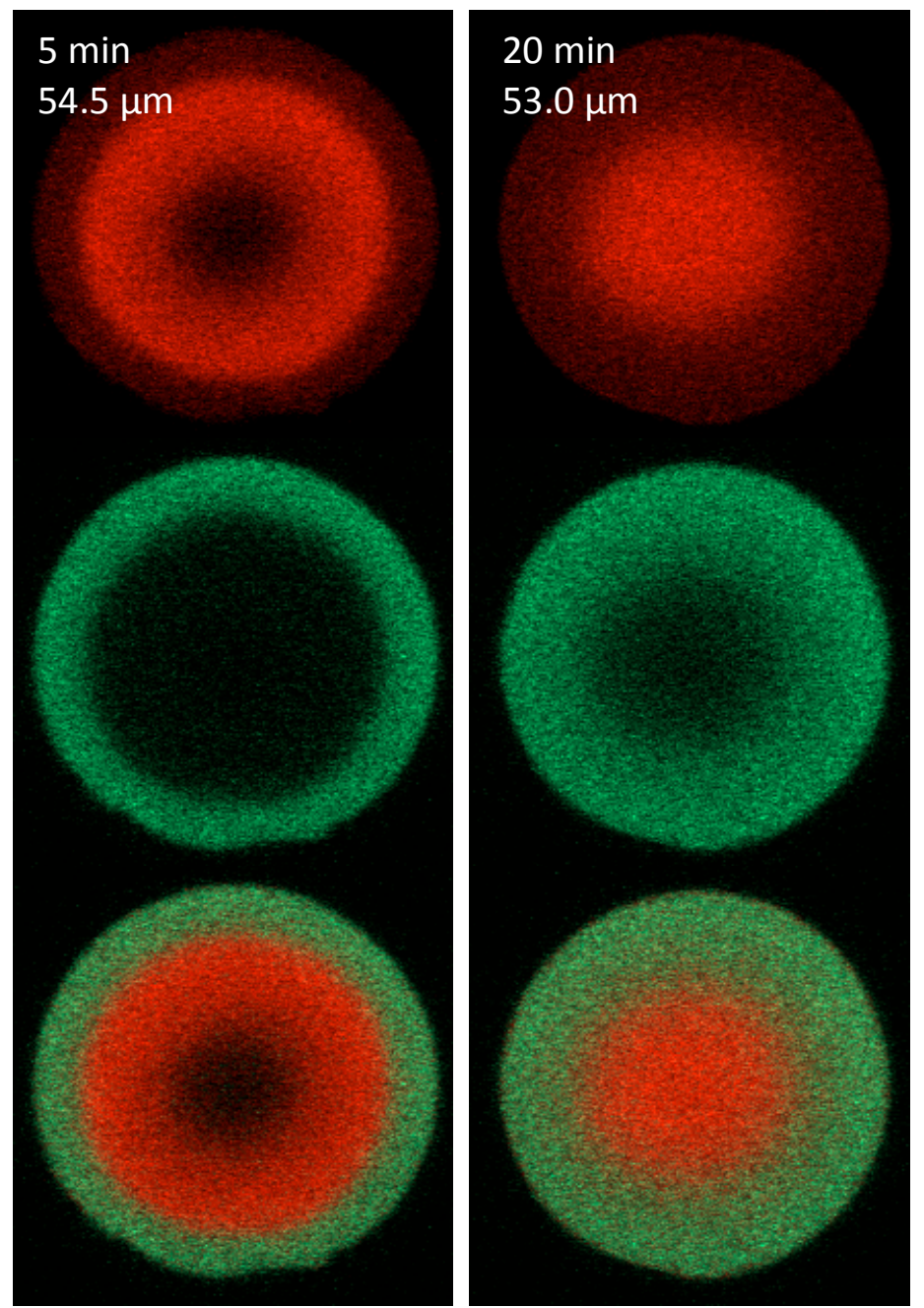

\section{$170 \mathrm{~min}$ \\ $54.1 \mu \mathrm{m}$}

Figure 7.7. Representative CLSM images of two-component adsorption of a mixture containing $1.09 \mathrm{mg} / \mathrm{mL} \mathrm{mAb}$ monomer and $0.88 \mathrm{mg} / \mathrm{mL} \mathrm{mAb}$ dimer in $10 \mathrm{mM} \mathrm{Na}{ }_{2} \mathrm{HPO}_{4}$ at $\mathrm{pH} 7.0$ with 60 $\mathrm{mM}$ added $\mathrm{NaCl}$. The top row (red) shows the monomer profile, the middle row (green) the dimer profile, and the bottom row a digital superposition of the monomer and dimer signals. Actual times and particle diameters are shown in the insets 

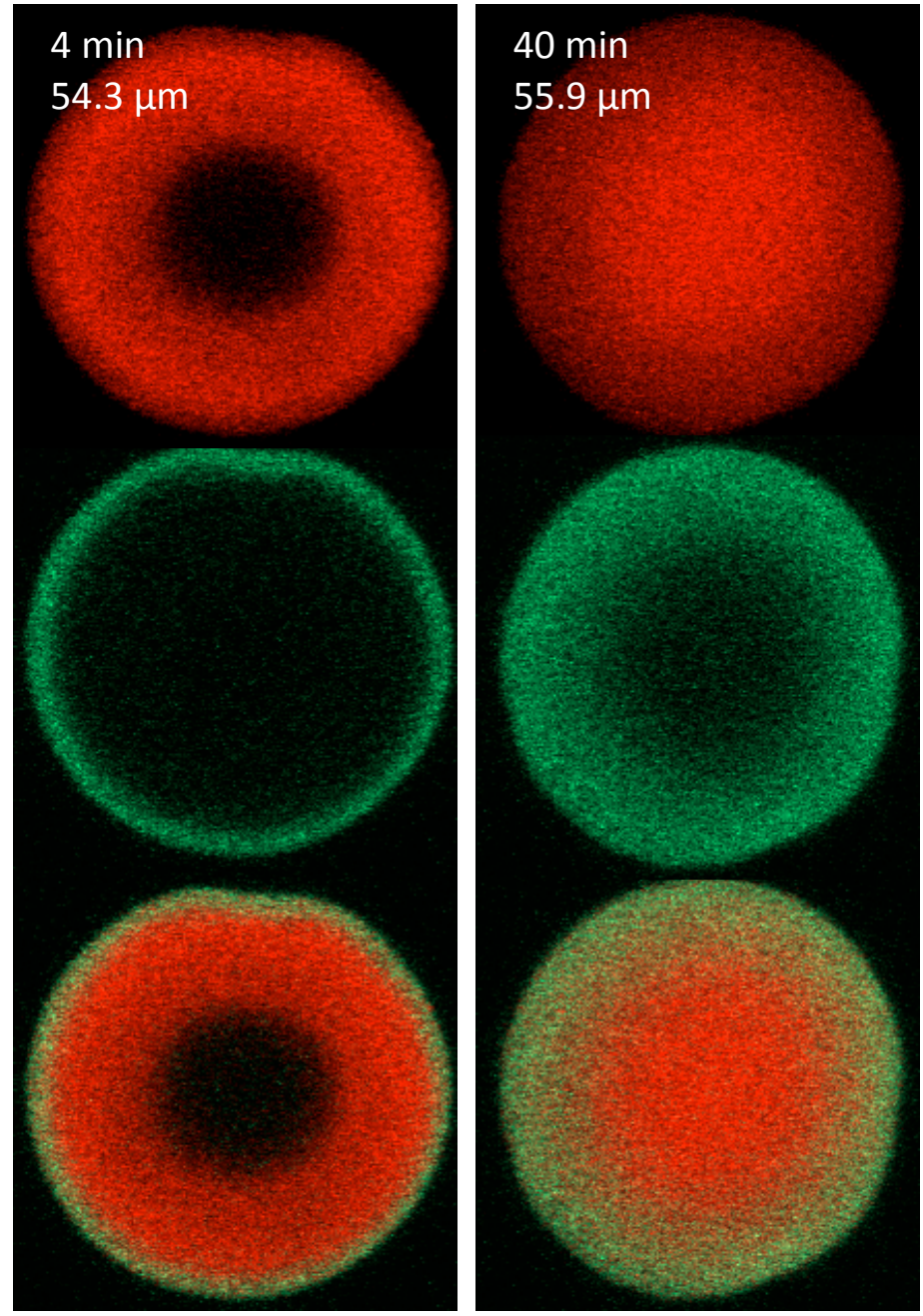

\section{$160 \mathrm{~min}$ \\ $55.1 \mu \mathrm{m}$}

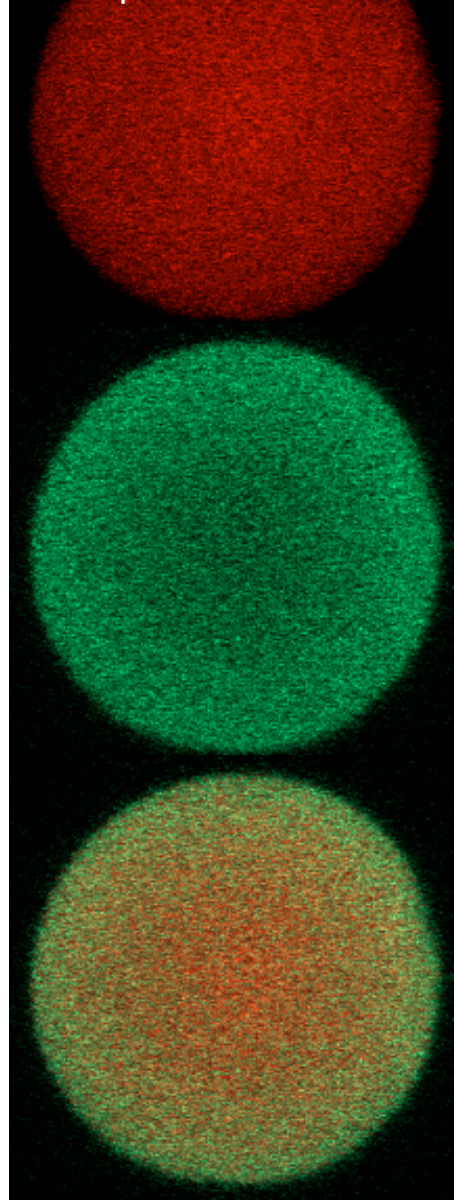

Figure 7.8. Representative CLSM images of two-component adsorption of a mixture containing $1.82 \mathrm{mg} / \mathrm{mL} \mathrm{mAb}$ monomer and $0.36 \mathrm{mg} / \mathrm{mL} \mathrm{mAb}$ dimer in $10 \mathrm{mM} \mathrm{Na}{ }_{2} \mathrm{HPO}_{4}$ at pH 7.0 with 60 $\mathrm{mM}$ added $\mathrm{NaCl}$. The top row (red) shows the monomer profile, the middle row (green) the dimer profile, and the bottom row a digital superposition of the monomer and dimer signals. Actual times and particle diameters are shown in the insets 
saturated zone from a layer saturated primarily with dimer with a small amount of monomer. Over time, these fronts disappear. First the protein-free core disappears and a monomersaturated core is formed. After that, the monomer-saturated core disappears, being gradually displaced by the advancing dimer front. At long times, the particle reaches equilibrium and contains an excess of bound dimer, consistent with the high selectivity determined from the equilibrium measurements at this salt concentration (Table 7.1).

Figure 7.8 shows an experiment for the same conditions as Fig. 7.7, but with a much lower ratio of dimer to monomer as might be encountered in a practical setting $(1.82 \mathrm{mg} / \mathrm{mL}$ monomer to $0.36 \mathrm{mg} / \mathrm{mL}$ dimer). The main difference in the results is that the monomer/dimer front advances much more slowly than in the case of Fig. 7.7. This occurs because the dimer concentration in the bulk solution, which is the driving force for mass transfer, is much lower. Despite the lower concentration, however, it is evident that the dimer still displaces the bound monomer via a similar (albeit slower) mechanism as that observed at the higher dimer concentration.

\subsection{Two-Component Kinetics Modeling}

Because the considered isotherm models are unable to provide a sufficiently accurate description of the competitive binding equilibrium and kinetics under low salt conditions (Section 7.1), detailed modeling of the two-component adsorption kinetics was not considered. However, a useful comparison of the rate data at the higher salt concentration can be made with the dual-shrinking core model presented by Martin et al. (2005). This model assumes that there is no kinetic limitation to binding, that the isotherms are rectangular for both adsorbed species, and that mass transfer is controlled by pore diffusion. Accordingly, the positions of the two adsorption fronts in the particle during simultaneous adsorption are described by the following equations:

$2 \rho_{s, M}^{3}-3 \rho_{s, M}^{2}+1=\left(\frac{q_{m, M}-q_{M}^{*}}{q_{m, M}}\right)\left[1+\frac{q_{D}^{*} D_{e, M} C_{M}}{\left(q_{m, M}-q_{M}^{*}\right) D_{e, D} C_{D}}\right] \frac{6 C_{D} D_{e, D} t}{q_{D}^{*} r_{p}^{2}}$ 
$2 \rho_{s, D}^{3}-3 \rho_{s, D}^{2}+1=\frac{6 C_{D} D_{e, D} t}{q_{D}^{*} r_{p}^{2}}$

where $\rho_{s, M}$ and $\rho_{s, D}$ are the dimensionless front positions of the monomer and of the monomer/dimer mixture, respectively, $q_{m, M}$ is the single component monomer binding capacity, $q_{M}^{*}$ and $q_{D}^{*}$ are the adsorbed concentration at equilibrium with the monomer/dimer mixture, $C_{M}$ and $C_{D}$ the monomer and dimer solution concentrations, and $D_{e, M}$ and $D_{e, D}$ are the effective pore diffusivities of the monomer and dimer, respectively. Figure 7.9 shows the dimensionless position of the two adsorption fronts observed during simultaneous adsorption for the experiments depicted in Figs. 7.7 and 7.8 in comparison with the lines calculated from Eqs. 7.4 and 7.5. The calculated curves are based on the values of $q_{m}$ and $D_{e}$ obtained for each species from the single component adsorption equilibrium and CLSM experiments (Sections 6.2 and 6.3), while the $q_{M}^{*}$ and $q_{D}^{*}$ were estimated based on the two-component adsorption equilibrium data (Table 7.1). The values used in the calculations are $q_{M}^{*}=25 \mathrm{mg} / \mathrm{mL}$ and $q_{D}^{*}=$ $78 \mathrm{mg} / \mathrm{mL}$ for Fig. $7.9 \mathrm{a}$, and $q_{M}^{*}=25 \mathrm{mg} / \mathrm{mL}$ and $q_{D}^{*}=70 \mathrm{mg} / \mathrm{mL}$ for Fig. $7.9 \mathrm{~b}$, which correspond to selectivities, $\alpha_{D, M}=4.3$ and 14 for (a) and (b), respectively. These values are only approximate since the experimental concentrations in the equilibrium experiments were not identical to those used in the experiments of Figs. 7.7 and 7.8. Despite the approximate nature of this estimation, it is apparent nonetheless, that the model captures the experimental trends very well for the monomer/dimer front in Fig. 7.9a and for the pure monomer front in Fig. 7.9b. The poorer agreement with the pure monomer front in (a) and with the monomer/dimer front in (b) suggests that the assumption implicit in the model that the isotherm is rectangular breaks down at the lower protein concentration of monomer in (a) and of the dimer in (b). A more precise prediction would seem to require a full description of the two-component isotherm behavior. 

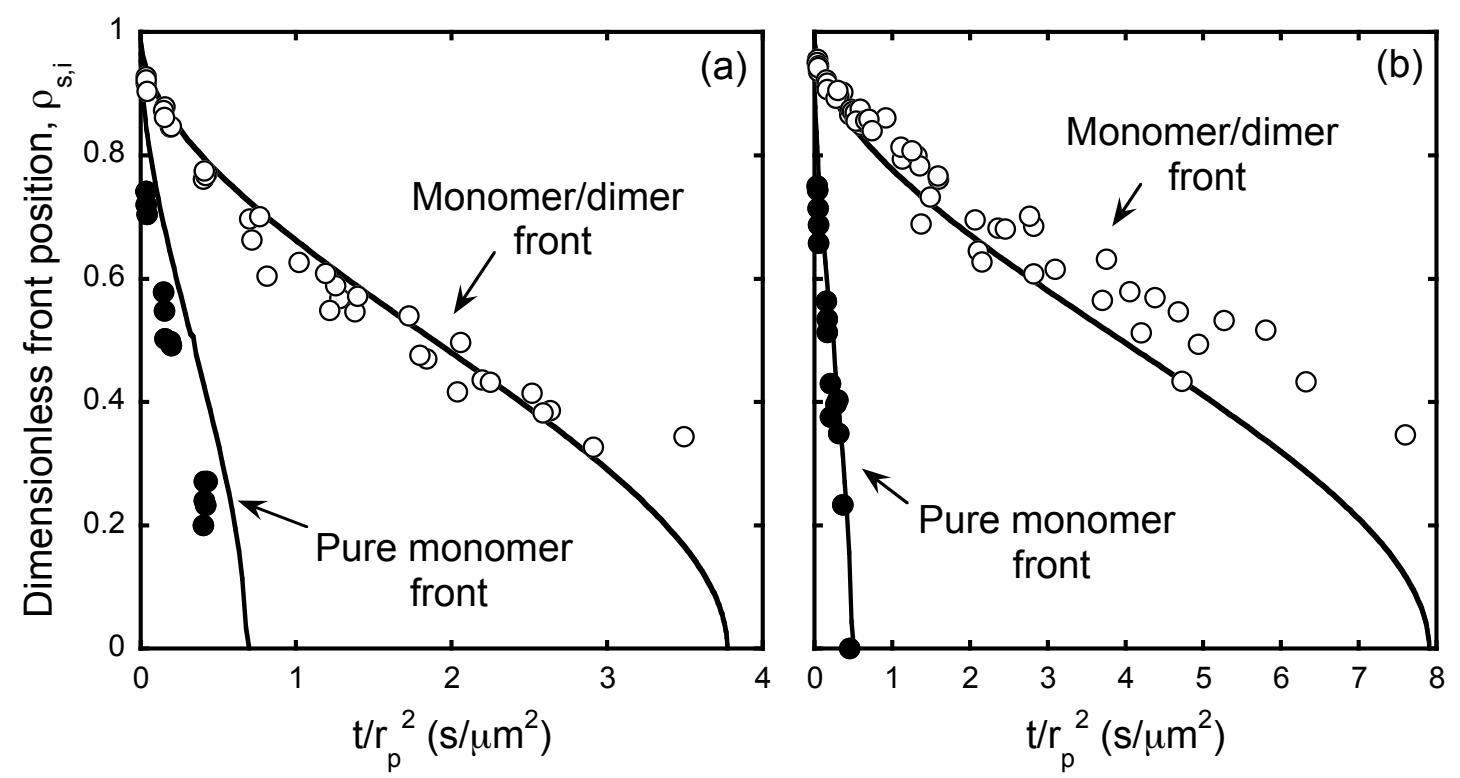

Figure 7.9. Dimensionless front position vs. reduced time for two-component adsorption in 10 $\mathrm{mM} \mathrm{Na}_{2} \mathrm{HPO}_{4}$ at pH 7.0 with $60 \mathrm{mM}$ added $\mathrm{NaCl}$ for the conditions of (a) Fig. 7.7 and (b) Fig. 7.8 with $C_{M}$ and $C_{D}$ the same as described in those Figs. Lines are based on Eqs. 7.4 and 7.5 using $q_{m}$ and $D_{e}$ values obtained from single component measurements and $q^{*}$ values estimated from the two-component equilibrium measurements (Table 7.1) 


\section{Frontal Analysis and Column Modeling of Monomer-Dimer Systems on Nuvia HR-S}

Chapters 6 and 7 investigated the adsorption equilibrium and kinetics of a monoclonal antibody $(\mathrm{mAb})$ and its soluble dimer in a macroporous cation exchange resin. Binding equilibrium and binding kinetics behaviors were investigated for monomer and dimer at both high and low protein loadings, and both individually and for the two-component system. It was found that conditions could be identified where (a) selective elution of the two species occurred in a salt gradient at low protein loads (Fig. 6.2), and (b) displacement of one species by the other occurred over relatively short time scales at high protein loads (Figs. 7.7 - 7.8). In general, effective displacement of the monomer by the dimer required intermediate salt concentrations. Almost no exchange between the two species was observed over reasonable time scales at low salt concentration, when binding is very strong. However, at intermediate salt concentrations, favorable exchange at rates that were nearly entirely limited by diffusional transport was observed.

Though carried out exclusively in batch mode, these results led to the conclusion that it might be possible to exploit the monomer-dimer exchange process to obtain separation by frontal analysis. In such a process, a monomer-dimer feed mixture would be continuously loaded to a column. Ideally, the more weakly bound species (the monomer in this case) would emerge from the column first, concentrated in a pure band as a result of competition with the dimer for binding sites. Then, immediately prior to dimer breakthrough, the column would be washed to recover the unbound monomer and eluted at high salt to remove the dimer along with any coadsorbed monomer.

Compared to bind-elute processes, a potential advantage of frontal analysis is that the column binding capacity is fully utilized, which, in turn, can lead to greater productivity. Its success depends, however, on having both selectivity and sufficiently fast kinetics so that the competitive binding process can occur over reasonable time scales. Since predicting purity and yield in such a process is more complicated, a model capable of describing the column dynamics is desirable. 
Protein separations by frontal analysis have been studied by prior authors for a few different systems. Weinbrenner and Etzel demonstrated the separation of BSA monomer and dimer by frontal loading on CEX membranes. Fargues, et al. showed the competitive breakthrough of a system of albumin and hemoglobin on hydroxyapatite supports. Garke et al. showed evidence of competitive binding of $\gamma$-globulin/lysozyme mixtures on a CEX column. These authors also developed a modified Langmuir model to describe competitive binding in batch adsorption, but did not provide the extension of the model to predict the column behavior. Hunter and Carta examined frontal analysis of BSA and BSA aggregate mixtures on AEX chromatography columns. Xu and Lenhoff also developed a model, based on colloidal energetics, to predict binary protein adsorption on CEX resins. While successful, the model was only tested against relatively small model proteins such as lysozyme, cytochrome $C$, and ribonuclease. A few studies have also recently been published on binary adsorption of antibodies. Liu et al. explored the frontal loading of mAbs on CEX columns, quantifying the breakthrough of aggregates and other impurities over a variety of stationary phases and loading conditions, but did not provide a mechanistic model to describe the separation. Tao et al. studied the separation of mAb charge variants by frontal analysis and provided a mechanistic model assuming full mass transfer control, but did not consider the separation of aggregates.

This chapter will first investigate experimentally the frontal analysis separation of an antibody monomer-dimer system at varying salt concentration and residence times, and additionally will develop a model to describe the separation, with the goal of being able to predict experimental conditions that provide an optimum balance of yield and purity.

\subsection{Experimental Breakthrough Curves}

Figure 8.1a and 8.1b show the single component breakthrough curves for monomer and dimer, respectively, in buffers containing 20 and $80 \mathrm{mM} \mathrm{Na}^{+}$(see Section 3.2.4). For both the monomer and dimer, breakthrough occurs earlier at $80 \mathrm{mM} \mathrm{Na}^{+}$because of the lower binding capacity. The dimer breakthrough curves are significantly shallower than those obtained for the 
monomer, a result consistent with the lower pore diffusivity of the dimer determined in Chapter 6 using confocal microscopy and non-binding pulse injections. The figures also show model predictions, which are discussed in Section 8.3.

Figures $8.2 a-8.6 a$ show representative binary breakthrough curves. Results are shown for load buffers containing $20,30,50,80$, and $120 \mathrm{mM} \mathrm{Na}^{+}$with a feed containing approximately 2.0 $\mathrm{mg} / \mathrm{mL}$ total protein with $70 \%$ monomer. In addition to the monomer and dimer breakthrough profiles, the outlet monomer purity, calculated as $C_{M} /\left(C_{M}+C_{D}\right)$, is also shown. The area between monomer and dimer curves is directly related to the selectivity. As seen in Fig. 8.2a, little separation occurs at $20 \mathrm{mM} \mathrm{Na}^{+}$. However, the separation improves dramatically at higher $\mathrm{Na}^{+}$concentrations with a well-defined band of purified monomer. For example, with $80 \mathrm{mM}$ $\mathrm{Na}^{+}$(Fig. 8.5a), fractions with monomer purity greater than $95 \%$ are obtained at the column outlet between approximately 25 and 75 CV, corresponding to feed loads between 50 and 150 $\mathrm{mg}$ total protein per $\mathrm{mL}$ of column. Each of the experimental binary breakthrough curves exhibits an overshoot of the monomer front above the feed concentration $\left(C_{M} / C_{M, F}>1\right)$, which confirms that the dimer displaces the bound monomer. The magnitude and duration of the overshoot is, however, dependent on the $\mathrm{Na}^{+}$concentration, ranging from a modest overshoot of very brief duration at $20 \mathrm{mM} \mathrm{Na}^{+}$, when binding is strongest and selectivity is apparently smallest, to a well defined overshoot at 50 and $80 \mathrm{Na}^{+}$, when binding is weaker but selectivity is higher.

Figures $8.2 b-8.6 b$ show the SEC results for the eluate pools, obtained by eluting the column with $1 \mathrm{M} \mathrm{NaCl}$ following each of the breakthrough runs, in comparison with those of the particular feed mixture used. Consistent with the breakthrough curves, at $20 \mathrm{mM} \mathrm{Na}^{+}$(Fig. $8.2 b)$, the eluate pool chromatogram is very similar to that of the feed. Conversely, much less monomer is found in the eluate pool compared to the feed for the $50 \mathrm{mM} \mathrm{Na}^{+}$run (Fig. 8.4b), and almost no monomer is found in the eluate pool at 80 (Fig. 8.5b) and $120 \mathrm{mM} \mathrm{Na}^{+}$(Fig. 8.6b). It is evident, however, that in addition to the dimer, some higher order species are also present in the eluate pool, as demonstrated by the early eluting peak in the SEC 

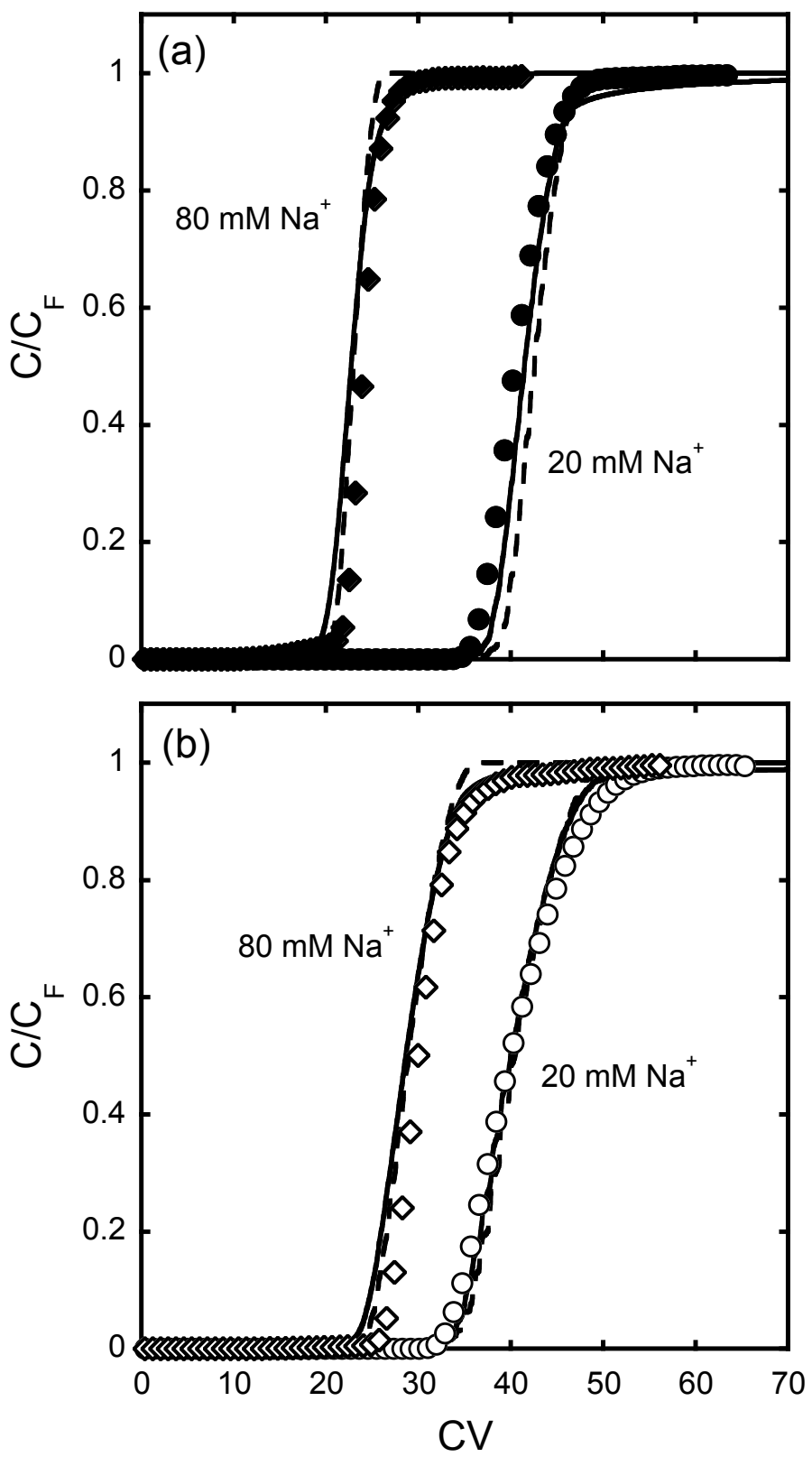

Figure 8.1. Single component breakthrough curves of (a) monomer and (b) dimer in $\mathrm{pH} 7$ load buffers containing 20 and $80 \mathrm{mM} \mathrm{Na}^{+}$. Feed concentrations were $2.0 \pm 0.1 \mathrm{mg} / \mathrm{mL}, u$ was 76 $\mathrm{cm} / \mathrm{h}$, column length was $4.4 \mathrm{~cm}$, and residence time was $3.5 \mathrm{~min}$. Dashed lines are model predictions neglecting any kinetic resistance to binding. Solid lines are fitted including a kinetic resistance to binding with rate constants given in Table 8.2 

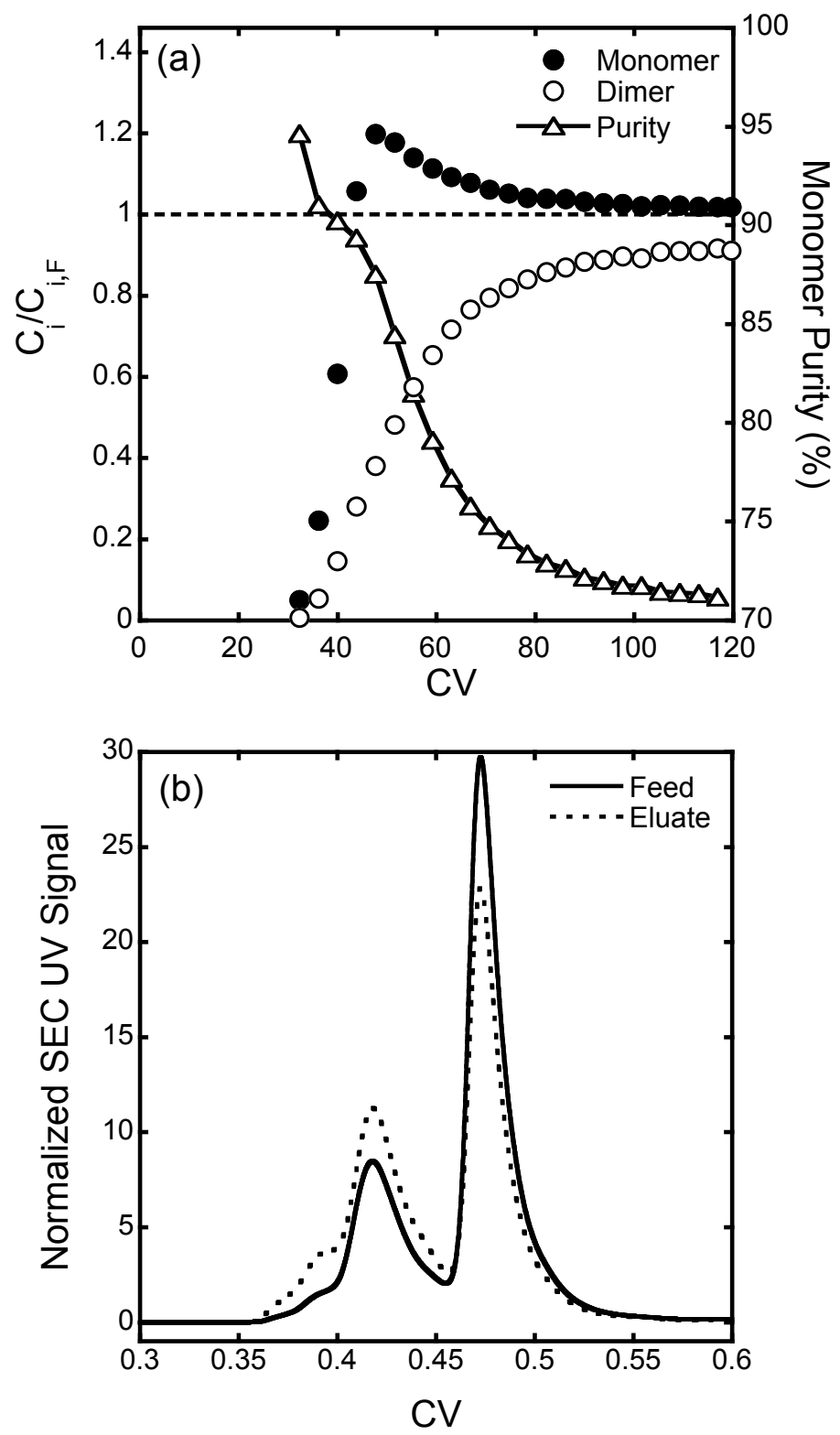

Figure 8.2. Binary breakthrough behavior of monomer-dimer mixture containing approximately $30 \%$ dimer in $\mathrm{pH} 7$ load buffer containing $20 \mathrm{mM} \mathrm{Na}^{+}$(a). SEC analysis of the experiment feed mixture (solid line) and the protein pool eluted in $1 \mathrm{M} \mathrm{NaCl}$ (dashed line) (b). $u$ was $150 \mathrm{~cm} / \mathrm{h}$, column length was $10.6 \mathrm{~cm}$, and residence time was $4.2 \mathrm{~min}$. Exact feed compositions are given in Table 8.1 

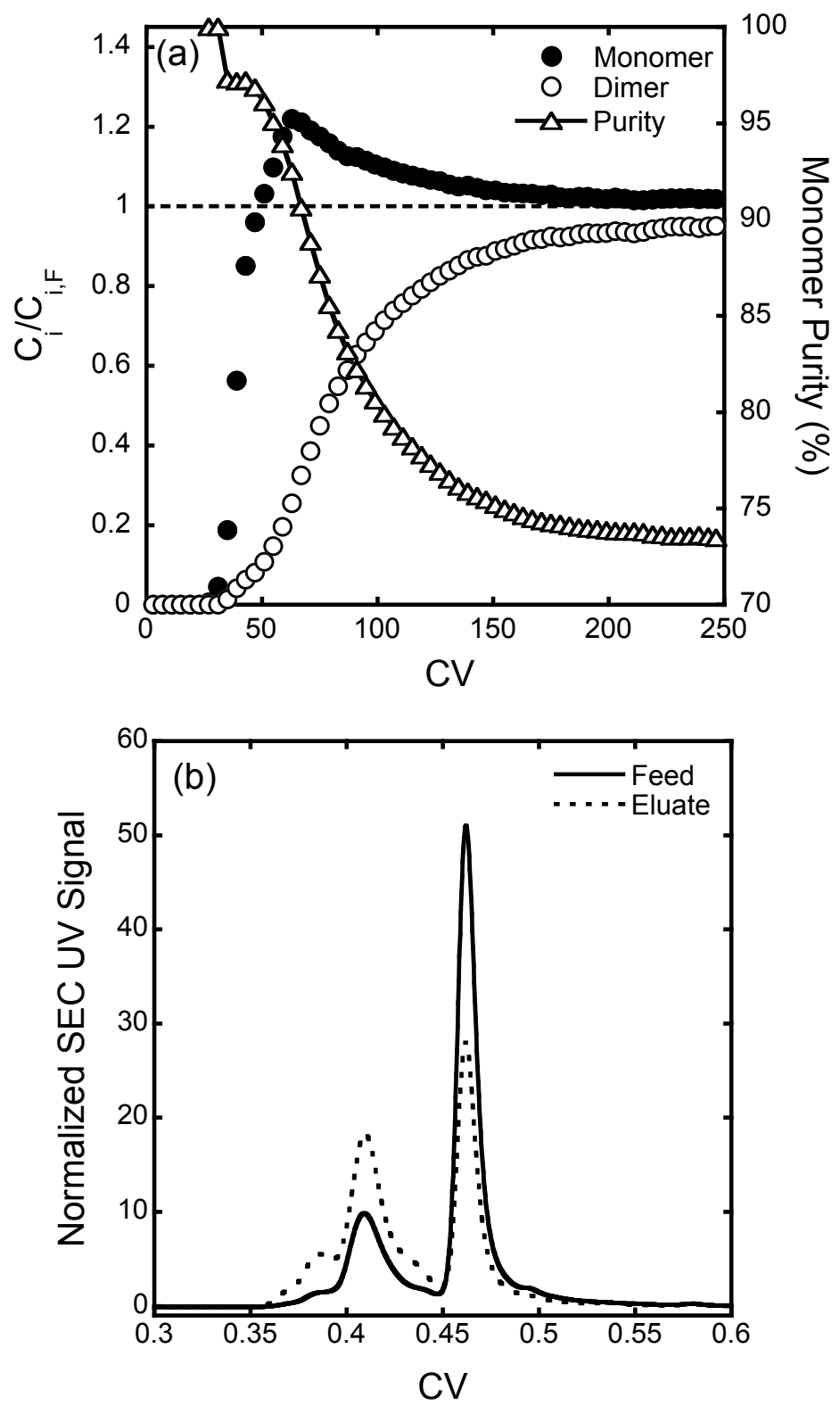

Figure 8.3. Binary breakthrough behavior of monomer-dimer mixture containing approximately $30 \%$ dimer in $\mathrm{pH} 7$ load buffer containing $30 \mathrm{mM} \mathrm{Na}^{+}$(a). SEC analysis of the experiment feed mixture (solid line) and the protein pool eluted in $1 \mathrm{M} \mathrm{NaCl}$ (dashed line) (b). $u$ was $76 \mathrm{~cm} / \mathrm{h}$, column length was $5.9 \mathrm{~cm}$, and residence time was 4.6 min. Exact feed compositions are given in Table 8.1 

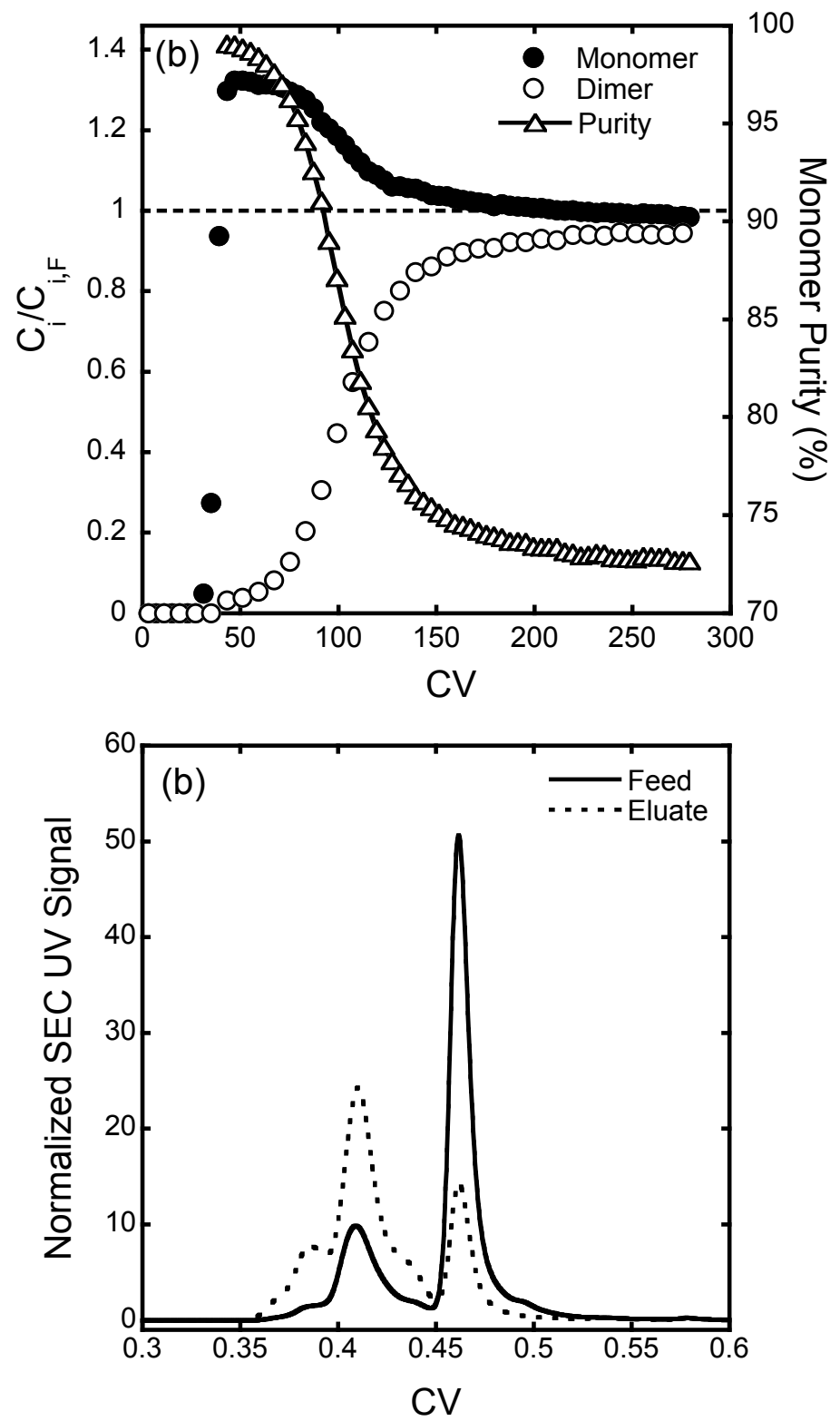

Figure 8.4. Binary breakthrough behavior of monomer-dimer mixture containing approximately $30 \%$ dimer in $\mathrm{pH} 7$ load buffer containing $50 \mathrm{mM} \mathrm{Na}^{+}$(a). SEC analysis of the experiment feed mixture (solid line) and the protein pool eluted in $1 \mathrm{M} \mathrm{NaCl}$ (dashed line) (b). $u$ was $76 \mathrm{~cm} / \mathrm{h}$, column length was $5.9 \mathrm{~cm}$, and residence time was 4.6 min. Exact feed compositions are given in Table 8.1 

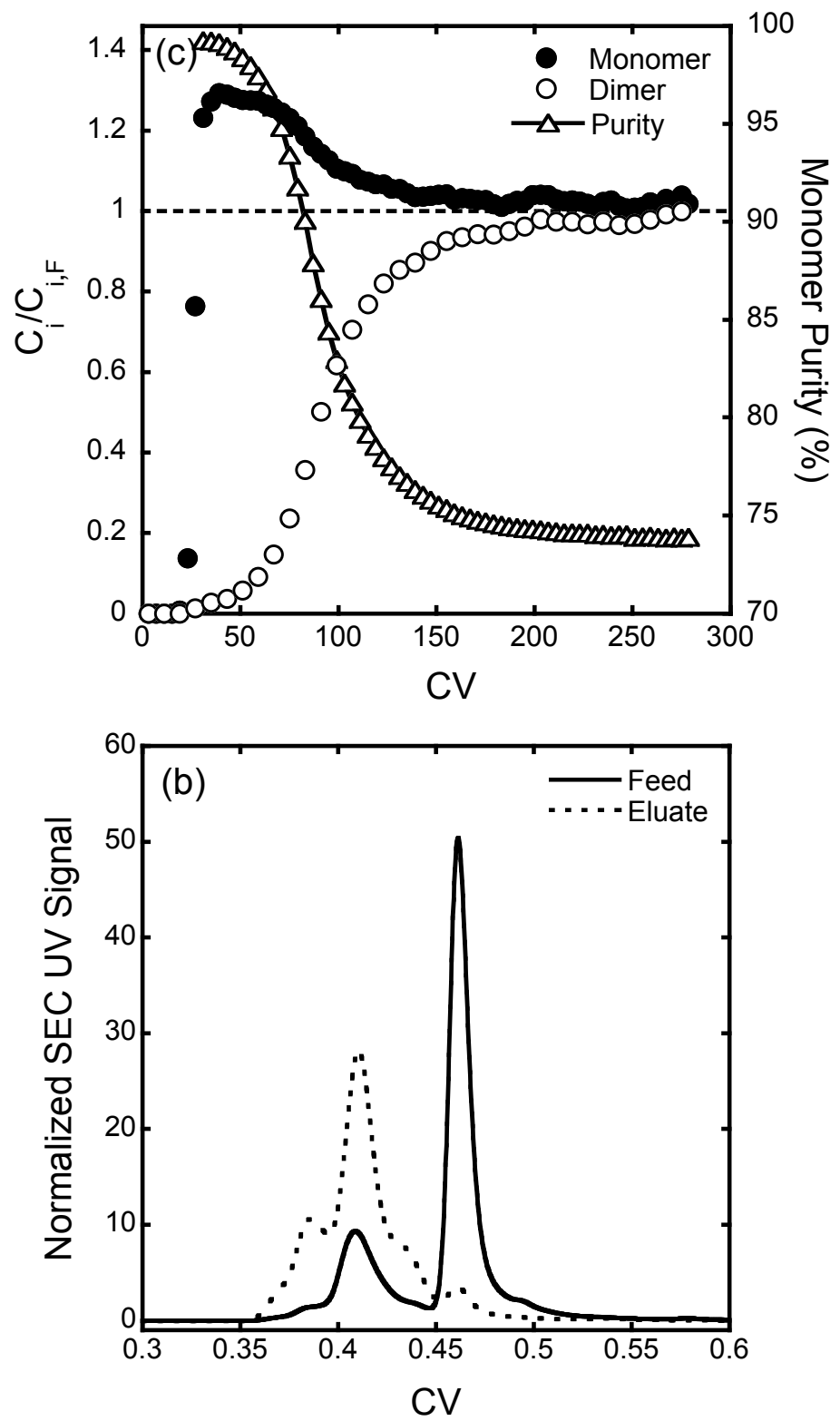

Figure 8.5. Binary breakthrough behavior of monomer-dimer mixture containing approximately $30 \%$ dimer in $\mathrm{pH} 7$ load buffer containing $80 \mathrm{mM} \mathrm{Na}^{+}$(a). SEC analysis of the experiment feed mixture (solid line) and the protein pool eluted in $1 \mathrm{M} \mathrm{NaCl}$ (dashed line) (b). $u$ was $76 \mathrm{~cm} / \mathrm{h}$, column length was $5.0 \mathrm{~cm}$, and residence time was 3.9 min. Exact feed compositions are given in Table 8.1 

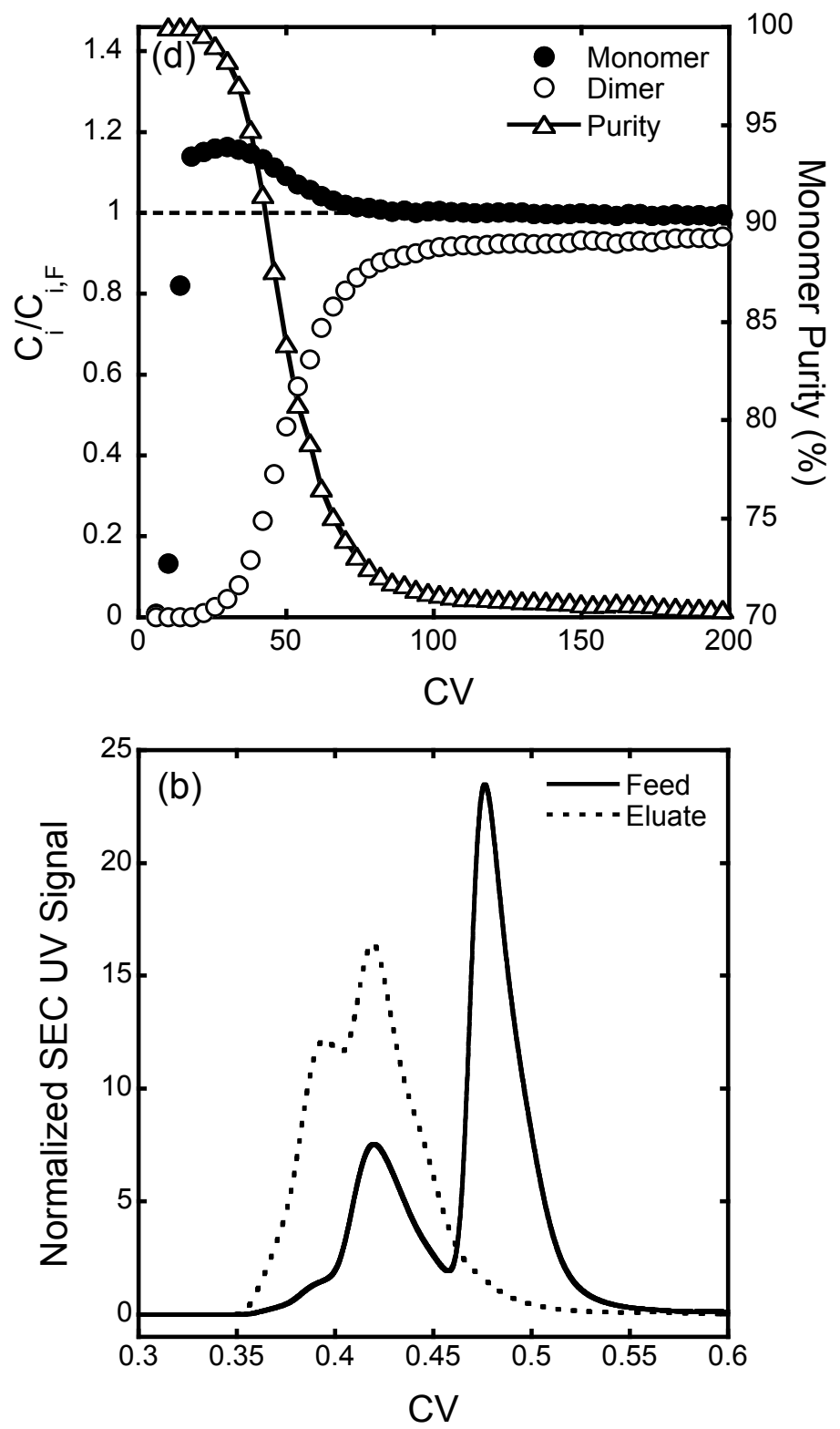

Figure 8.6. Binary breakthrough behavior of monomer-dimer mixture containing approximately $30 \%$ dimer in $\mathrm{pH} 7$ load buffer containing $120 \mathrm{mM} \mathrm{Na}^{+}$(a). SEC analysis of the experiment feed mixture (solid line) and the protein pool eluted in $1 \mathrm{M} \mathrm{NaCl}$ (dashed line) (b). $u$ was $76 \mathrm{~cm} / \mathrm{h}$, column length was $5.6 \mathrm{~cm}$, and residence time was $4.4 \mathrm{~min}$. Exact feed compositions are given in Table 8.1 
chromatograms. These species are present as impurities in the original dimer sample and are concentrated on the resin during the frontal analysis process.

\subsection{Binding Capacities}

The monomer and dimer equilibrium binding capacities were obtained directly by integrating the breakthrough curves. Under local equilibrium conditions, the pure component breakthrough curve would consist of a shock emerging from the column at:

$C V_{s h}=\varepsilon+(1-\varepsilon) \frac{q^{*}\left(C_{F}\right)}{C_{F}}$

where $\varepsilon$ is the extraparticle (or external) porosity, $q^{*}\left(C_{F}\right)$ is the bound protein concentration in equilibrium with the feed (in units of $\mathrm{mg}$ per $\mathrm{mL}$ of particle volume), $C_{F}$ is the protein feed concentration, and $C V$ represents the effluent volume divided by the column volume (Ruthven 1983; Carta \& Jungbauer 2010). Accordingly, the equilibrium binding capacities can be calculated from the area above each breakthrough curve with the following equation:

$q^{*}\left(C_{F}\right)=\frac{\int_{0}^{\infty}\left(C_{F}-C\right) d C V}{(1-\varepsilon)}-\frac{\varepsilon C_{F}}{(1-\varepsilon)}$

where $C$ is the actual effluent protein concentration, and the remaining variables are as defined in Eq. 8.1. For the binary feed, again under local equilibrium conditions and assuming that the dimer can displace the monomer, the breakthrough curve would comprise two shocks with an intermediate plateau of pure monomer at a concentration $C_{M, 1}$. These two shocks would occur at $C V_{s h, 1}$ and $C V_{s h, 2}$ given by (Ruthven 1983; Carta \& Jungbauer 2010):

$$
\begin{aligned}
& C V_{s h, 1}=\varepsilon+(1-\varepsilon) \frac{q_{M}^{*}\left(C_{M, 1}, 0\right)}{C_{M, 1}} \\
& C V_{s h, 2}=\varepsilon+(1-\varepsilon) \frac{q_{M}^{*}\left(C_{M, F}, C_{D, F}\right)-q_{M}^{*}\left(C_{M, 1}, 0\right)}{C_{M, F}-C_{M, 1}}=\varepsilon+(1-\varepsilon) \frac{q_{D}^{*}\left(C_{M, F}, C_{D, F}\right)}{C_{D, F}}
\end{aligned}
$$

where $C_{M, F}$ and $C_{D, F}$ are the monomer and dimer feed concentrations, and the remaining variables are as defined in Eq. 8.1. As seen in Figs. 8.2 - 8.6, the experimental profiles are 
diffuse and only roughly approximate the local equilibrium shocks. Nevertheless, the values of $q_{M}^{*}\left(C_{M, 1}, 0\right), q_{M}^{*}\left(C_{M, F}, C_{D, F}\right)$, and $q_{D}^{*}\left(C_{M, F}, C_{D, F}\right)$ can still be calculated approximately from Eqs. 8.3 and 8.4 by substituting the centers of mass of the breakthrough curves of pure monomer and monomer/dimer feed in place of $C V_{s h, 1}$ and $C V_{s h, 2}$.

The results of these calculations are shown in Table 8.1 where they are compared with predictions based on single component isotherm measurements using the steric mass action (SMA) law model (Brooks \& Cramer 1992):

$q_{i}^{*}=\frac{K_{e, i}\left[q_{0}-\left(z_{M}+\sigma_{M}\right) q_{M}-\left(z_{D}+\sigma_{D}\right) q_{D}\right]^{z_{i}} C_{i}}{C_{N a^{+}}^{z_{i}}}$

where $q_{0}$ is the resin charge density, $K_{e, i}$ is the equilibrium constant, $z_{i}$ is the effective protein binding charge, and $\sigma_{i}$ is the steric hindrance parameter. The values of the parameters appearing in this equation, $q_{0}, K_{e, i}, z_{i}$, and $\sigma_{i}$, were those obtained in Chapter 6 from a global fit of single component isotherms over different $\mathrm{Na}^{+}$concentrations (Table 6.1).

As seen in Table 8.1, the overshoot concentrations, $C_{M, 1}$, and the corresponding $q^{*}$-values derived from the pure monomer front are in reasonable agreement with the SMA isotherm values at all $\mathrm{Na}^{+}$concentrations. However, agreement for the mixture $q^{*}$-values, derived from the monomer/dimer front, is highly dependent on the $\mathrm{Na}^{+}$concentration. At $20 \mathrm{mM} \mathrm{Na}{ }^{+}$, when protein binding is strongest, there is very poor agreement with the SMA model. On the other hand, at higher $\mathrm{Na}^{+}$concentrations, when protein binding is weaker, the agreement improves significantly and becomes quantitative for both monomer and dimer at 80 and $120 \mathrm{mM} \mathrm{Na}^{+}$. This result is consistent with the conclusions reached in Chapter 7, where, based on batch isotherm data, it was shown that the SMA model was only suitable at relatively high $\mathrm{Na}^{+}$ concentrations. In fact, based on Eq. 8.5, the monomer-dimer selectivity, $\alpha_{D, M}=q_{D}^{*} C_{M} / q_{M}^{*} C_{D}$, is predicted to be inversely proportional to $C_{\mathrm{Na}^{+}}^{z_{D^{-}} z_{M}}$. Since the dimer effective charge, $z_{D}$, is greater than that of the monomer, $z_{M}$, the SMA model predicts lower selectivity for the dimer 


\begin{tabular}{|c|c|c|c|c|c|c|c|c|}
\hline $\begin{array}{c}C_{N a^{+}} \\
(\mathrm{mM})\end{array}$ & $\begin{array}{c}C_{M, F} \\
(\mathrm{mg} / \mathrm{mL})\end{array}$ & $\begin{array}{c}C_{D, F} \\
(\mathrm{mg} / \mathrm{mL})\end{array}$ & \multicolumn{2}{c|}{$\begin{array}{c}C_{M, 1} / q_{M}^{*}\left(C_{M, 1}\right) \\
(\mathrm{mg} / \mathrm{mL})\end{array}$} & \multicolumn{2}{c|}{$\begin{array}{c}q_{M}^{*}\left(C_{M, F}, C_{D, F}\right) \\
(\mathrm{mg} / \mathrm{mL})\end{array}$} & \multicolumn{2}{|c|}{$\begin{array}{c}q_{D}^{*}\left(C_{M, F}, C_{D, F}\right) \\
(\mathrm{mg} / \mathrm{mL})\end{array}$} \\
\cline { 4 - 9 } & & $\begin{array}{c}\mathrm{BT} \\
\text { Curve }\end{array}$ & $\begin{array}{c}\text { SMA } \\
\text { Model }\end{array}$ & $\begin{array}{c}\mathrm{BT} \\
\text { Curve }\end{array}$ & $\begin{array}{c}\text { SMA } \\
\text { Model }\end{array}$ & $\begin{array}{c}\text { BT } \\
\text { Curve }\end{array}$ & $\begin{array}{c}\text { SMA } \\
\text { Model }\end{array}$ \\
\hline 20 & 2.0 & 0 & - & - & 130 & 140 & - & - \\
\hline 20 & 0 & 2.1 & - & - & - & - & 140 & 150 \\
\hline 20 & 1.6 & 0.73 & $2.0 / 130$ & $2.3 / 140$ & 91 & 8.9 & 71 & 140 \\
\hline 30 & 1.4 & 0.52 & $1.6 / 110$ & $1.8 / 130$ & 70 & 8.8 & 83 & 130 \\
\hline 50 & 1.3 & 0.52 & $1.8 / 110$ & $1.8 / 100$ & 25 & 7.8 & 96 & 110 \\
\hline 80 & 2.0 & 0 & - & - & 76 & 74 & - & - \\
\hline 80 & 0 & 2.0 & - & - & - & - & 99 & 95 \\
\hline 80 & 1.4 & 0.52 & $1.8 / 77$ & $1.9 / 73$ & 9 & 7 & 87 & 83 \\
\hline 120 & 1.4 & 0.64 & $1.7 / 30$ & $1.8 / 36$ & 5 & 5 & 67 & 53 \\
\hline
\end{tabular}

Table 8.1. Equilibrium binding capacities obtained from the single and binary breakthrough experiments compared with predictions from the SMA model 
at higher $\mathrm{Na}^{+}$concentration. This trend is observed experimentally at relatively high $\mathrm{Na}^{+}$, but not at all at low $\mathrm{Na}^{+}$concentrations.

\subsection{Breakthrough Modeling}

The model used to describe the breakthrough curves for both single and two-component systems is based on two main assumptions. The first is that plug flow is assumed with axial dispersion simulated through the numerical dispersion introduced by a backwards-finite difference discretization of the relevant material balance equation. The second is that intraparticle transport is assumed to be controlled by pore-diffusion. The first of these assumptions is based on the fact that the reduced velocity, $v^{\prime}=v d_{p} / D_{0}$, where $v$ is the interstitial velocity, $d_{p}$ is the particle diameter, and $D_{0}$ is the protein solution diffusivity, is well above 500 for the conditions studied. In this case, mass transfer, rather than axial dispersion, dominates band broadening (Carta et al. 2005). The second assumption is based on confocal microscopy imaging results in Chapter 6 , showing that single component adsorption at low $\mathrm{Na}^{+}$ concentrations followed very closely the shrinking core model. The effective pore diffusivities, $D_{e}=8.0 \times 10^{-8}$ and $4.1 \times 10^{-8} \mathrm{~cm}^{2} / \mathrm{s}$ for the mAb monomer and mAb dimer, respectively, were determined, and are, as expected, 5 to 10 times lower than the corresponding free solution diffusivities as a result of diffusional hindrance. External mass transfer is also neglected based on the large value of the Biot number, $B i=k_{f} r_{p} / D_{e}$, where $k_{f}$ is the boundary layer mass transfer coefficient and $r_{p}$ is the particle radius. Using $k_{f}$-values estimated from engineering correlations (Wilson \& Geankoplis 1966) gave $B i$-values well in excess of 40, demonstrating that the external resistance is negligible. Finally, although the single component adsorption kinetics is expected to be fast, a kinetic resistance to binding can be expected for the binary case since this involves the competitive binding of molecules with large differences in size and binding charge. Accordingly, our model also includes a kinetic contribution to binding, which is represented by the kinetic expression of the SMA model.

The model equations along with initial and boundary conditions are as follows: 
$\varepsilon \frac{\partial C_{i}}{\partial t}-\left.\frac{3(1-\varepsilon) D_{e, i}}{r_{p}} \frac{\partial c_{i}}{\partial r}\right|_{r=r_{p}}+u \frac{\partial C_{i}}{\partial x}=0$

$\left.C_{i}\right|_{t=0}=0,\left.\quad C_{i}\right|_{x=0}=C_{i, F}$

$\varepsilon_{p} \frac{\partial c_{i}}{\partial t}+\frac{\partial q_{i}}{\partial t}=\frac{D_{e, i}}{r^{2}} \frac{\partial}{\partial r}\left(r^{2} \frac{\partial c_{i}}{\partial r}\right)$

$\left.c_{i}\right|_{t=0}=0,\left.\frac{\partial c_{i}}{\partial r}\right|_{r=0}=0,\left.\quad c_{i}\right|_{r=r_{p}}=C_{i}$

$\frac{\partial q_{i}}{\partial t}=k_{i}\left\{c_{i}\left[q_{0}-\sum_{j}\left(z_{j}+\sigma_{j}\right) q_{j}\right]^{z_{i}}-\frac{q_{i} C_{N a^{+}}^{z_{i}}}{K_{e, i}}\right\}$

$\left.q_{i}\right|_{t=0}=0$

In these equations, $C_{i}$ and $c_{i}$ are the protein concentrations in the mobile phase and within the particle pores, respectively, $u$ is the mobile phase superficial velocity, $x$ is the column axial coordinate, $q_{i}$ is the local bound protein concentration at a radial position $r$ within the particle, $\varepsilon_{p}$ is the intraparticle porosity, and $k_{i}$ is a rate constant accounting for the finite rate of exchange between protein and $\mathrm{Na}^{+}$counterions. The model equations (Eqs. 8.6-8.8) were solved numerically for both one and two-component systems by spatially discretizing Eq. 8.6 and 8.7 by finite differences and integrating the resulting system of time-dependent ODEs using the ode15s variable order differential equation solver in MATLAB R2015a (The Mathworks, Natick, MA, USA). A more detailed description of the equation discretizations can be found in Appendix A. The SMA model parameters were obtained from single component isotherm data determined in Chapter 6. Additional model parameters (e.g. $\varepsilon, u, r_{p}$ ) were set to match experimental conditions and measured resin properties (see Chapter 4). The rate constant values, $k_{M}$ and $k_{D}$, for monomer and dimer, respectively, were fitted to the experimental breakthrough profiles. The model parameters are summarized in Table 8.2.

Model predictions of the single component breakthrough curves are shown in Fig. 8.1. Two cases are shown, one without a kinetic resistance to binding, i.e. assuming a completely mass transfer controlled process (dashed lines) and one including a kinetic resistance as described by 
Eq. 8.8 (solid lines). For the mass transfer controlled case, the values of $k_{M}$ and $k_{D}$ were chosen to be sufficiently high that further increasing them did not change the shape of the model predicted breakthrough curves. In this scenario, the concentrations at each local discretization point in the numerical solution scheme are essentially under equilibrium conditions as described by the multi-component SMA isotherm of Eq. 8.5. Conversely, for the case where the kinetic resistance is included, $k_{M}$ and $k_{D}$ were adjusted to minimize the difference between experimental and model profiles. As seen in this figure, the difference between the two cases, with and without kinetic resistance to binding, is very small, suggesting that the exchange between either monomer or dimer for $\mathrm{Na}^{+}$counterions on the CEX surface is fast.

Model predictions for the binary breakthrough curves are shown in Figs. 8.7-8.11. As before, the dashed lines are predictions without a kinetic resistance while the solid lines include the resistance according to Eq. 8.8. For the cases of 20 and $80 \mathrm{mM} \mathrm{Na}^{+}$, Figs. 8.7 and 8.10, respectively, the values of the kinetic constants, $k_{M}$ and $k_{D}$, are the same as those obtained by fitting the corresponding single component breakthrough curves. For the other cases (Figs. 8.8, 8.9, and 8.11), $k_{M}$ and $k_{D}$ were adjusted to provide the best possible agreement with the experimental curves. As seen in Fig. 8.7, whether a kinetic resistance is included or not, model predictions deviate grossly from the experimental profiles. Without a kinetic resistance, the model predicted separation is much worse. This occurs because, as discussed previously, the SMA model fails to predict the two-component equilibrium for these very strong binding conditions. In this case, no set of $k_{M}$ and $k_{D}$ values results in predicted breakthrough curves that follow the experimental ones. Agreement can only be obtained if the equilibrium model matches the experimental $q^{*}$ values (see Table 8.1). $\mathrm{As} \mathrm{Na}^{+}$is increased to $30 \mathrm{mM}$ (Fig. 8.8), the case without a kinetic resistance still deviates grossly from the experimental profiles, but the case with a kinetic resistance begins to resemble the shape of the experimental curves. Further, as seen in Figs. 8.9-8.11, higher $\mathrm{Na}^{+}$concentrations lead to model predicted curves that are much closer to the experimental ones. Moreover, it can be seen that the difference between curves calculated with and without a kinetic resistance become much closer to each 


\begin{tabular}{|c|c|c|}
\hline \multicolumn{3}{|c|}{ General Parameters } \\
\hline \multicolumn{2}{|l|}{ Column length } & See figure captions \\
\hline \multicolumn{2}{|c|}{ Superficial velocity, $u$} & See figure captions \\
\hline \multicolumn{2}{|c|}{ Particle radius, $r_{p}(\mu \mathrm{m})$} & 26 \\
\hline \multicolumn{2}{|c|}{ Column extraparticle porosity, $\varepsilon$} & 0.38 \\
\hline \multicolumn{2}{|c|}{ Resin intraparticle porosity, $\varepsilon_{p}$} & 0.50 \\
\hline \multicolumn{3}{|c|}{ Individual Protein Parameters } \\
\hline & Monomer & Dimer \\
\hline$D_{e}\left(\mathrm{~cm}^{2} / \mathrm{s}\right)$ & $8.0 \times 10^{-8}$ & $4.1 \times 10^{-8}$ \\
\hline$q_{0}$ & \multicolumn{2}{|c|}{132} \\
\hline$z$ & 10.2 & 14.8 \\
\hline$\sigma$ & 111 & 229 \\
\hline$K_{e}$ & 104 & $1.33 \times 10^{4}$ \\
\hline \multicolumn{3}{|c|}{ Rate Parameters for Eq. 8.8} \\
\hline$C_{\mathrm{Na}^{+}}(\mathrm{mM})$ & $k_{M}\left(\mathrm{mM}^{-2} \mathrm{~s}^{-1}\right)$ & $k_{D}\left(\mathrm{mM}^{-2} \mathrm{~s}^{-1}\right)$ \\
\hline 20 & $1 \times 10^{-17}$ & $1.3 \times 10^{-23}$ \\
\hline 30 & $3 \times 10^{-18}$ & $1 \times 10^{-20}$ \\
\hline 50 & $3 \times 10^{-20}$ & $1 \times 10^{-24}$ \\
\hline 80 & $1 \times 10^{-21}$ & $4 \times 10^{-29}$ \\
\hline 120 & $2 \times 10^{-23}$ & $3 \times 10^{-31}$ \\
\hline
\end{tabular}

Table 8.2. Parameters used in model calculations of Eqs. 8.6-8.8. Individual protein parameters from experiments in Chapter 6 


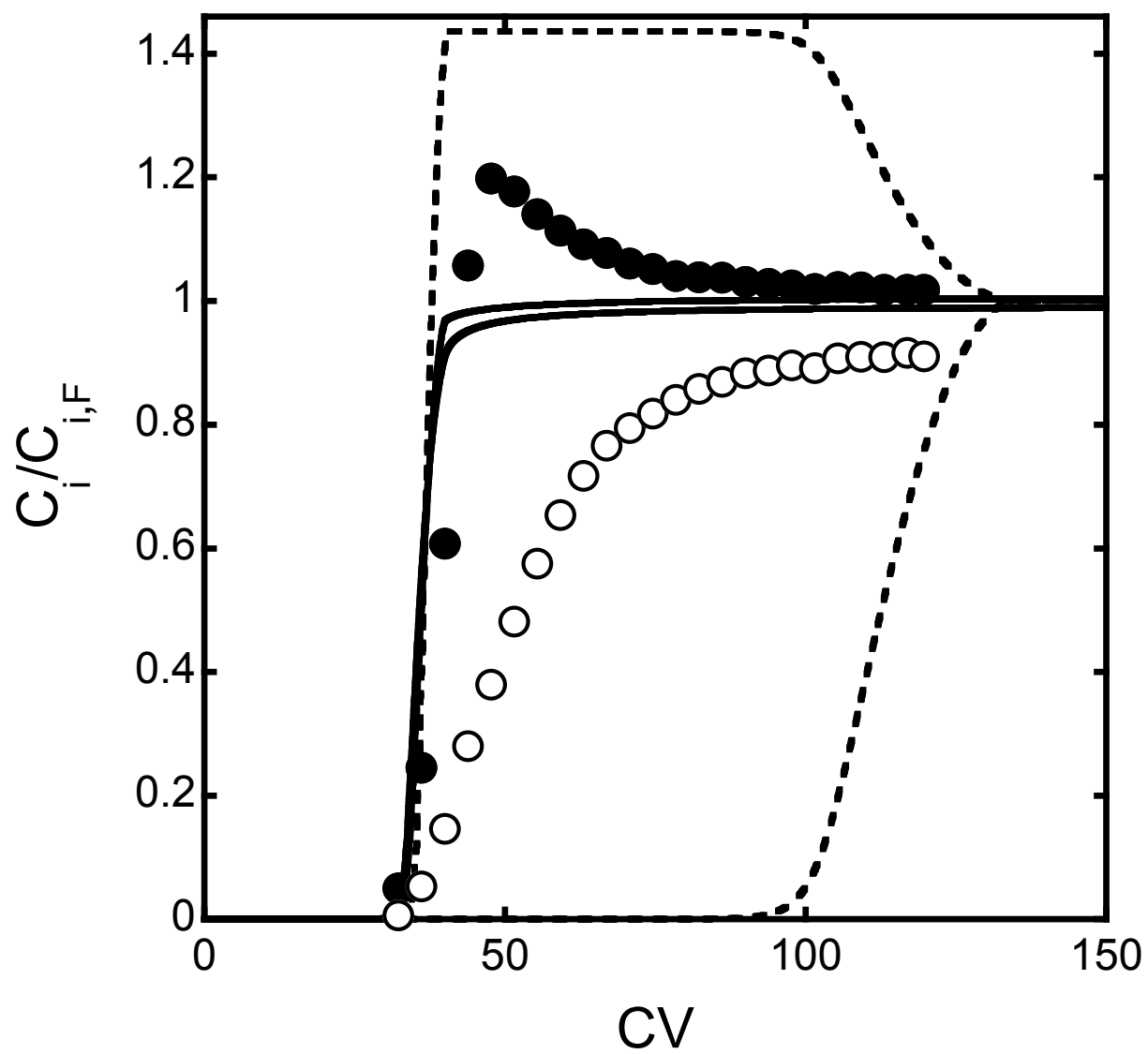

Figure 8.7. Comparison of binary breakthrough curves at $20 \mathrm{mM} \mathrm{Na}^{+}$with predictions based on Eqs. 8.6-8.8 using the SMA model (Eq. 8.5) to describe competitive binding of monomer and dimer. Experimental conditions are the same as in Fig. 8.2. Dashed lines show predictions without including a kinetic resistance to binding. Solid lines include a kinetic resistance to binding according to Eq. 8.8 with rate constants given in Table 8.2 


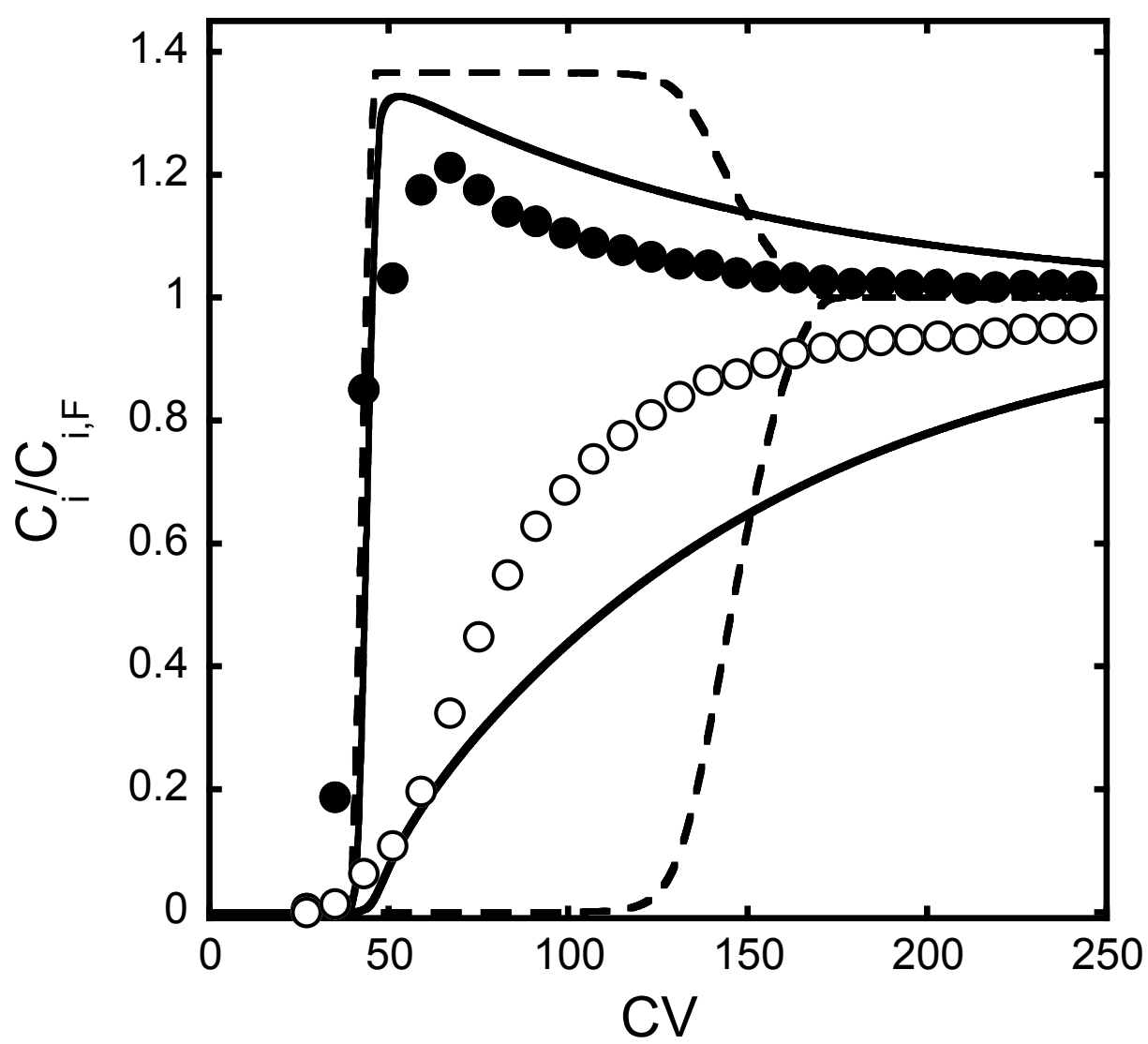

Figure 8.8. Comparison of binary breakthrough curves at $30 \mathrm{mM} \mathrm{Na}^{+}$with predictions based on Eqs. 8.6-8.8 using the SMA model (Eq. 8.5) to describe competitive binding of monomer and dimer. Experimental conditions are the same as in Fig. 8.3. Dashed lines show predictions without including a kinetic resistance to binding. Solid lines include a kinetic resistance to binding according to Eq. 8.8 with rate constants given in Table 8.2 


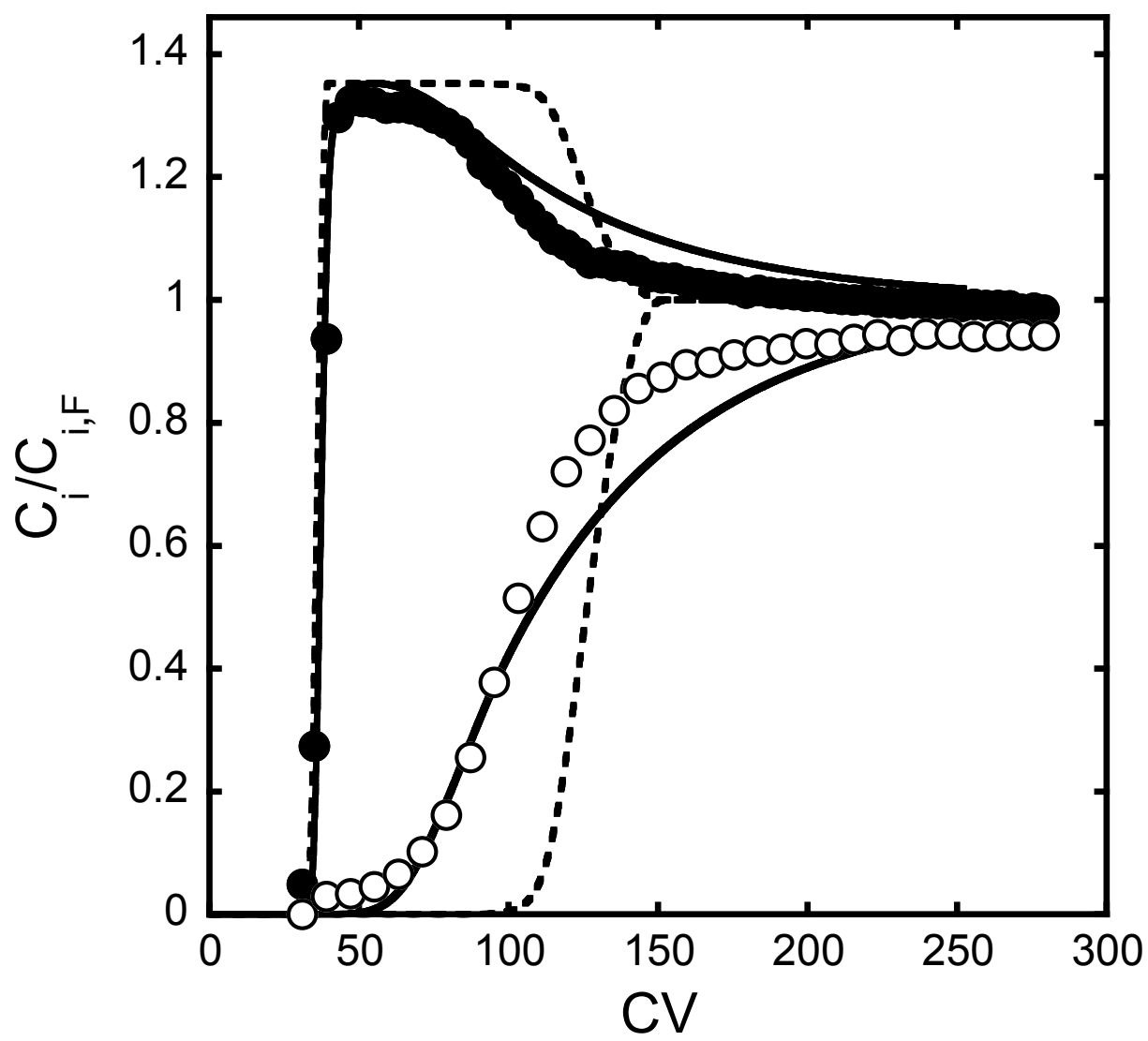

Figure 8.9. Comparison of binary breakthrough curves at $50 \mathrm{mM} \mathrm{Na}^{+}$with predictions based on Eqs. 8.6-8.8 using the SMA model (Eq. 8.5) to describe competitive binding of monomer and dimer. Experimental conditions are the same as in Fig. 8.4. Dashed lines show predictions without including a kinetic resistance to binding. Solid lines include a kinetic resistance to binding according to Eq. 8.8 with rate constants given in Table 8.2 


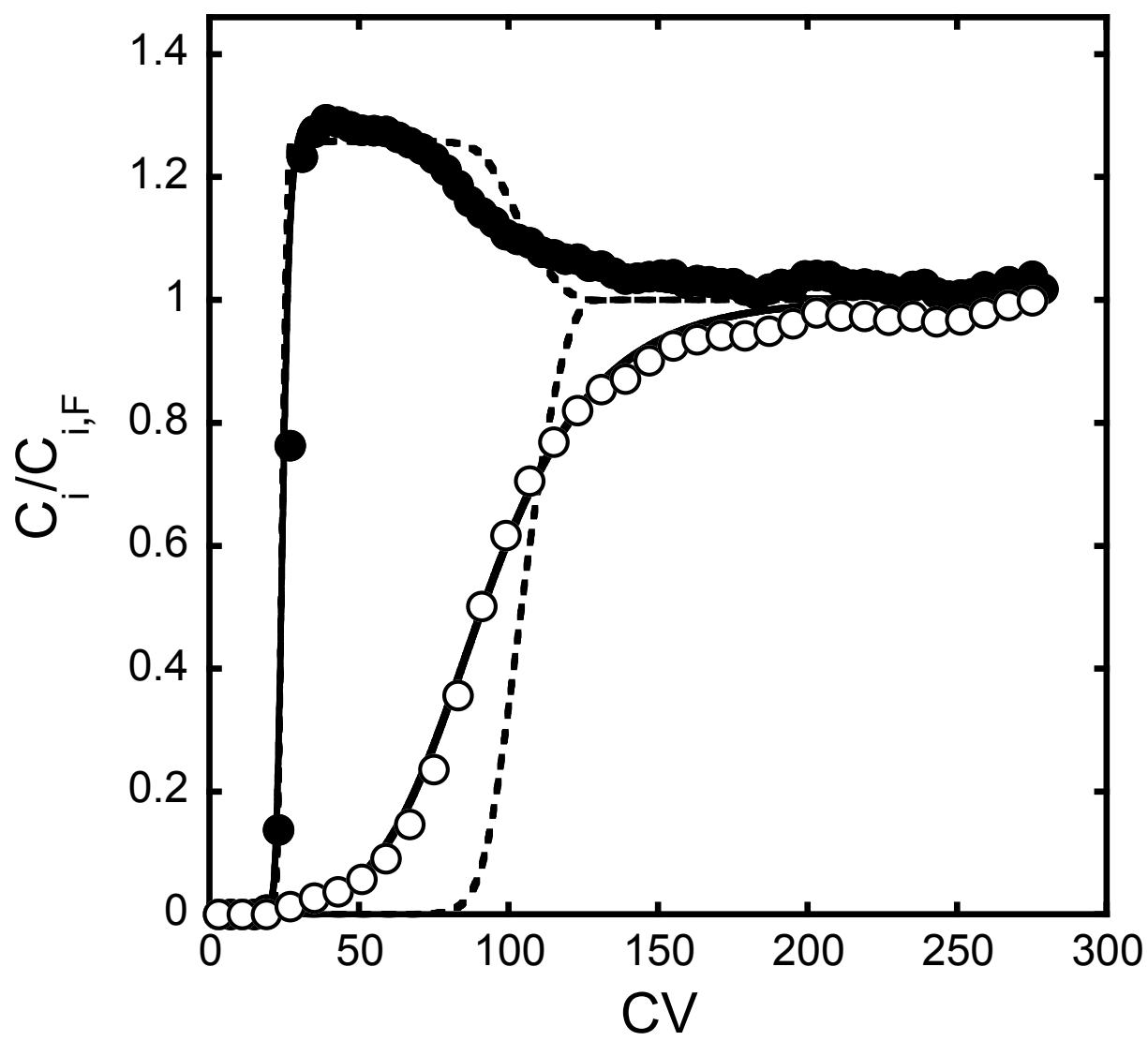

Figure 8.10. Comparison of binary breakthrough curves at $80 \mathrm{mM} \mathrm{Na}^{+}$with predictions based on Eqs. 8.6 - 8.8 using the SMA model (Eq. 8.5) to describe competitive binding of monomer and dimer. Experimental conditions are the same as in Fig. 8.5. Dashed lines show predictions without including a kinetic resistance to binding. Solid lines include a kinetic resistance to binding according to Eq. 8.8 with rate constants given in Table 8.2 


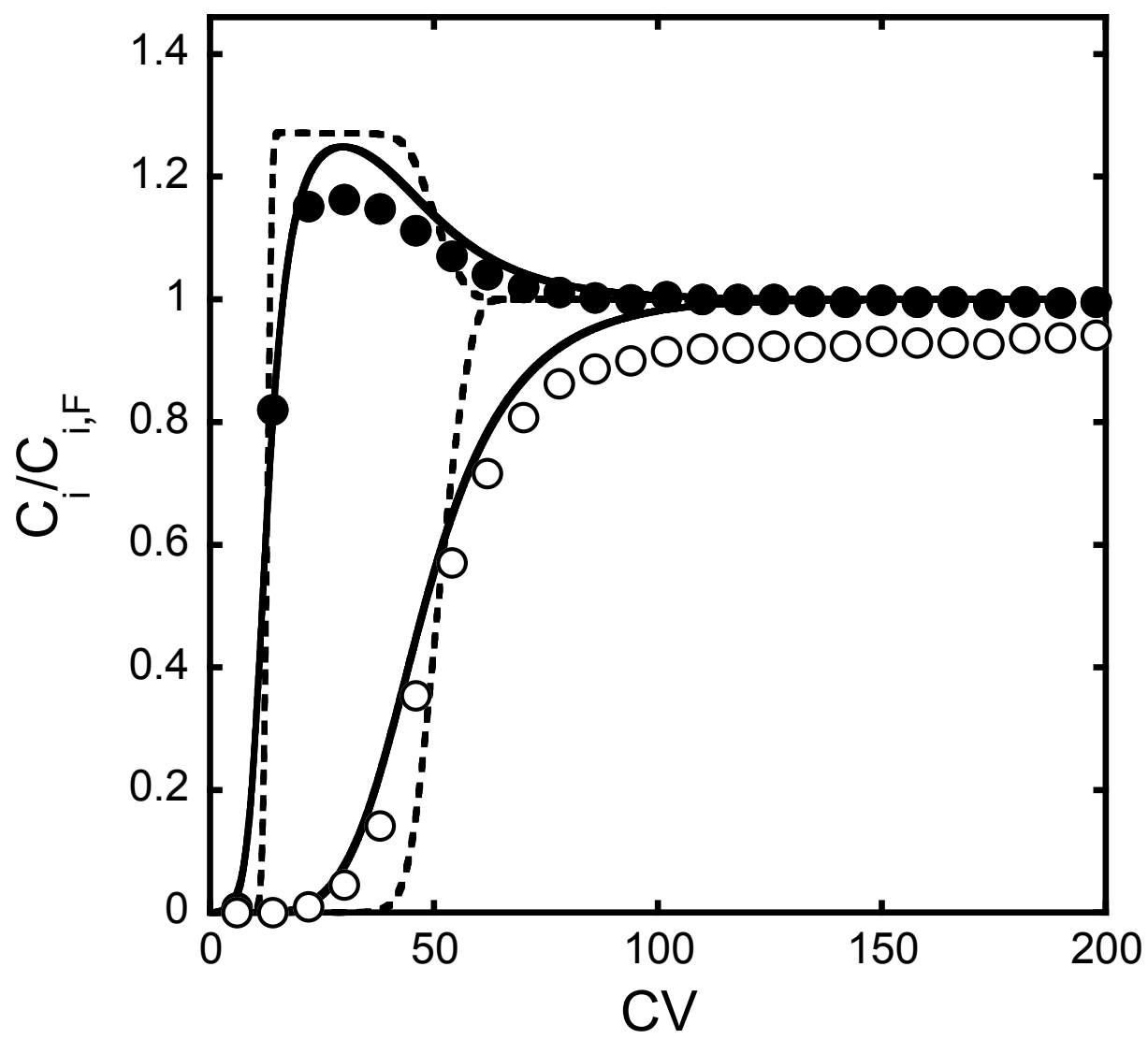

Figure 8.11. Comparison of binary breakthrough curves at $120 \mathrm{mM} \mathrm{Na}^{+}$with predictions based on Eqs. 8.6 - 8.8 using the SMA model (Eq. 8.5) to describe competitive binding of monomer and dimer. Experimental conditions are the same as in Fig. 8.6. Dashed lines show predictions without including a kinetic resistance to binding. Solid lines include a kinetic resistance to binding according to Eq. 8.8 with rate constants given in Table 8.2 
other as the $\mathrm{Na}^{+}$concentration is increased. In turn, this suggests that as the $\mathrm{Na}^{+}$concentration increases, the frontal analysis process becomes increasingly mass transfer controlled and, thus, predictable without including the kinetic resistance to binding.

A final consideration is whether the model developed in this work can (a) predict the effect of load composition and (b) provide results that are consistent with prior observation of intraparticle concentration profiles during co-adsorption of monomer-dimer mixtures shown in Chapter 7. The first consideration is of obvious practical value, while the second is important to confirm the physical validity of the model that has been developed as, usually, agreement with single-particle level measurements provides a stricter test of the validity of the model assumptions. Figure 8.12 compares model predictions with the experimental two-component breakthrough curves at $80 \mathrm{mM} \mathrm{Na}^{+}$obtained with a feed containing $85 \%$ monomer. Additionally, the experiment was run on a different length column with a significantly shorter residence time than the previous $80 \mathrm{mM} \mathrm{Na}^{+}$experiment. The experimental SEC chromatograms of the feed and eluate pool are also shown (Fig. 8.12b). As seen in Fig. 8.12a, the model prediction (adjusting only the feed concentrations and column length) is in excellent agreement with the experimental concentration and purity profiles. In this case, since the initial monomer purity is higher, purity levels above $95 \%$ are obtained between about 30 and $130 \mathrm{CV}$, corresponding to the range 60 to $260 \mathrm{mg}$ total protein loaded per $\mathrm{mL}$ of column volume. Note that, in agreement with the model, the monomer concentration overshoot is smaller than in the previous cases where the dimer content of the feed was higher. SEC analysis of the eluate pool (Fig. 8.12b) shows that at the end of the loading process the resin contains primarily bound dimer with a certain amount of higher order aggregates that were concentrated in the column as a result of their stronger binding.

Figures 8.13 - 8.15 compare model predictions with the intraparticle concentration profiles obtained by confocal microscopy for the co-adsorption of the monomer-dimer mixture at $\mathrm{pH}$ 7.0 at various experimental conditions. These concentration profiles were taken from Chapter 7, with Figs. 8.13, 8.14, and 8.15 corresponding to the model fits of the experiments depicted in 

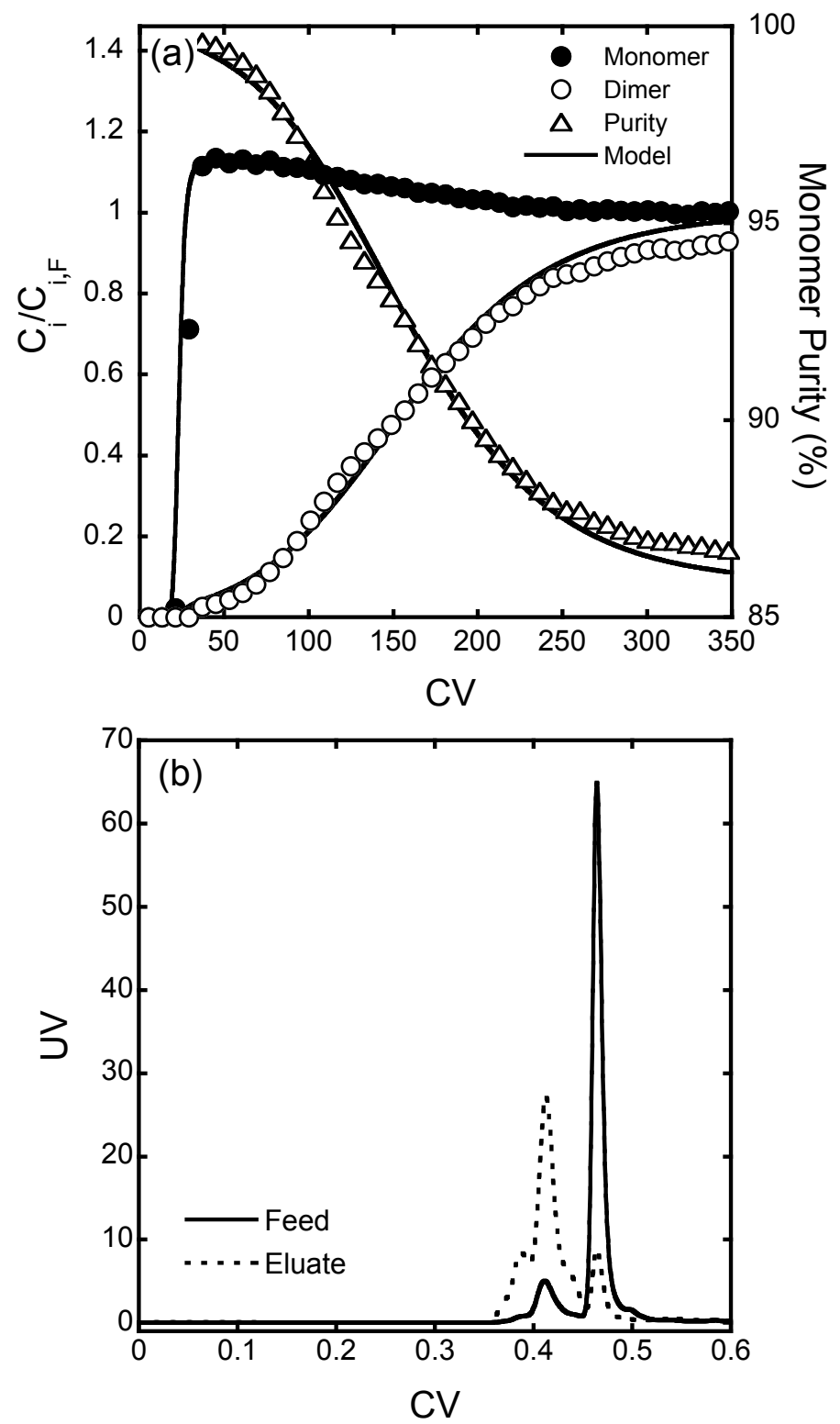

Figure 8.12. Binary breakthrough behavior of a $2 \mathrm{mg} / \mathrm{mL}$ monomer-dimer mixture containing approximately $15 \%$ dimer in $\mathrm{pH} 7$ load buffer with $80 \mathrm{mM} \mathrm{Na}^{+}$(a). SEC analysis of the experiment feed mixture (solid line) and the protein pool eluted in $1 \mathrm{M} \mathrm{NaCl}$ (dashed line) (b). $u$ was $76 \mathrm{~cm} / \mathrm{h}$, column length was $3.0 \mathrm{~cm}$, and residence time was $2.4 \mathrm{~min}$. Lines in (a) are predictions based on Eqs. 8.6 - 8.8 including a kinetic resistance to binding with rate constants in Table 8.2 
Figs. 7.3, 7.7, and 7.8, respectively. The radial intraparticle concentration profiles were obtained from the grey-scale intensities using ImageJ 1.42q image analysis software (Schneider et al. 2012) assuming that the red and green fluorescence intensities were proportional to the bound protein concentrations. Since the exact relationship between fluorescence intensity and protein concentration is unknown and varies with the microscope settings, the intensity curves were normalized so that their radial average would match the model-predicted profiles. Fig. 8.13 shows the case at $20 \mathrm{mM} \mathrm{Na}^{+}$, which, for the model with no kinetic resistance, predicts nearly complete displacement. However, with the kinetic resistance, profiles are obtained where even at long times (Fig. 8.13c), the predicted monomer concentration is higher than that of the dimer. Additionally, the model can capture the similar speeds of the monomer and dimer adsorption fronts into the particle first observed in Chapter 7. Though the model predicts qualitatively the single-particle behavior, deficiencies in the model are exacerbated in a column setting, resulting in the poorer fit of Fig. 8.7. However, as seen in Figs. 8.14 and 8.15 for the 80 $\mathrm{mM} \mathrm{Na}^{+}$cases, the model predicts nearly quantitatively the shape of the experimental intensity profiles, further confirming its validity. In agreement with the data, the model predicts relatively fast kinetics with relatively diffuse intraparticle concentration profiles, with the more weakly bound monomer rapidly advancing toward the center of the particle ahead of the dimer early on (Figs. 8.14a and 8.15a), followed by much slower rates later (Figs. 8.14b-c and 8.15b-c), with the dimer gradually displacing the bound monomer. It is notable that even after $160 \mathrm{~min}$, a substantial amount of monomer remains bound as a result of the relatively slow kinetics of competitive binding. This feature is clearly captured by the model. 

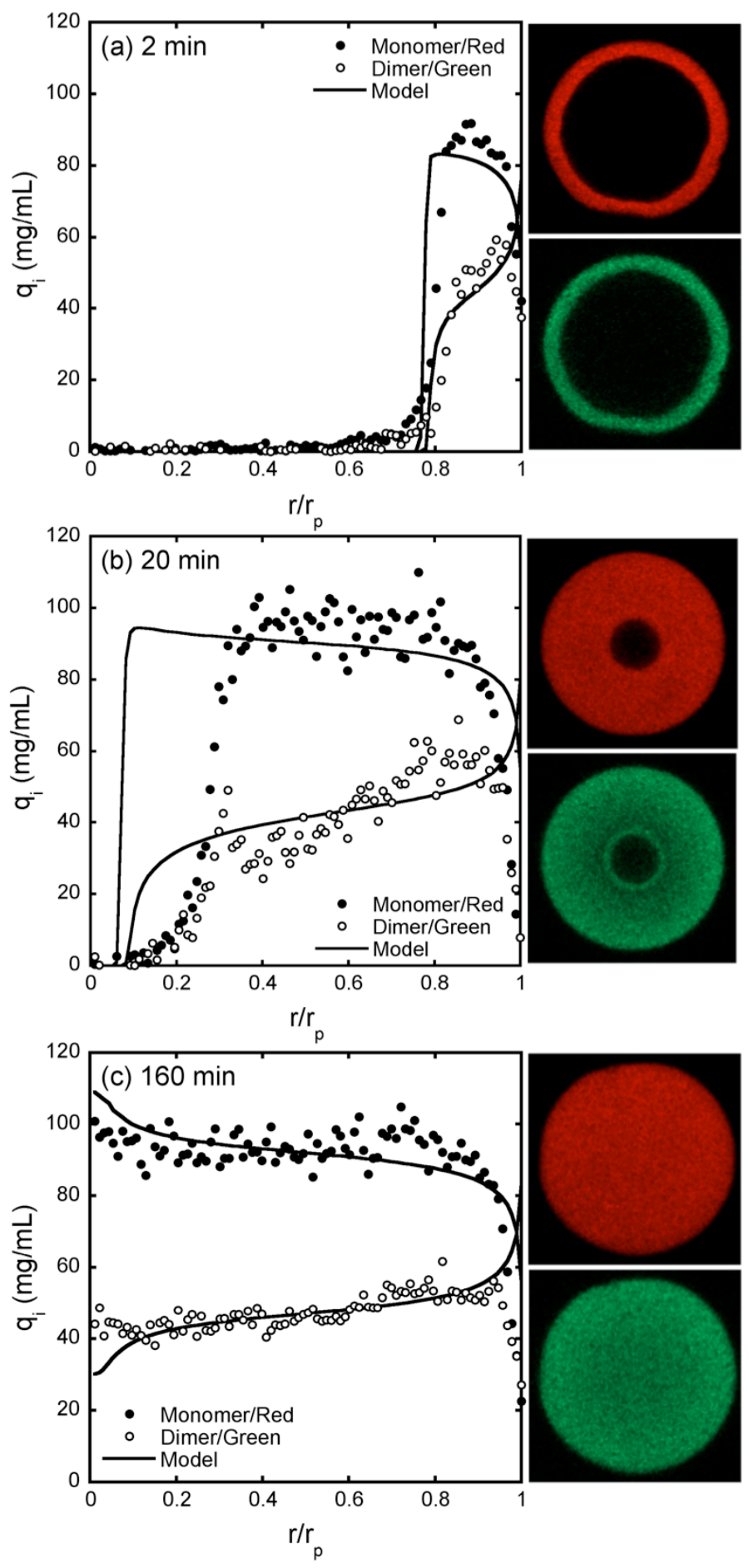

Figure 8.13. Comparison of experimental intraparticle concentration profiles of Fig. 7.3 (in 20 $\mathrm{mM} \mathrm{Na}^{+}$) with model predictions based on Eqs. 8.7 - 8.8. Solid and open circles are normalized fluorescence intensity profiles of the monomer and dimer, respectively. Lines are model predictions based on original experimental conditions 

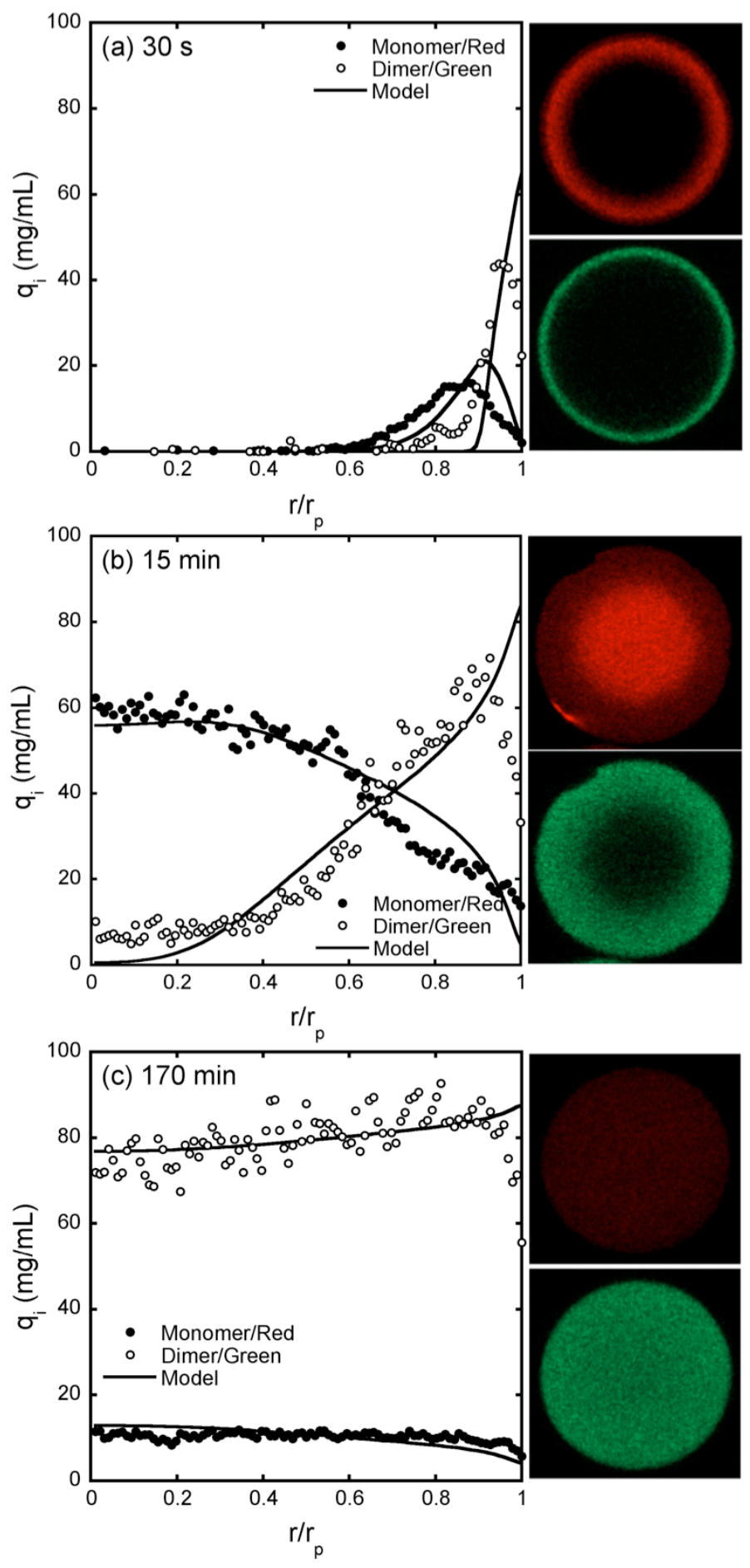

Figure 8.14. Comparison of experimental intraparticle concentration profiles of Fig. 7.7 with 80 $\mathrm{mM} \mathrm{Na}^{+}$with model predictions based on Eqs. 8.7 - 8.8. Solid and open circles are normalized fluorescence intensity profiles of the monomer and dimer, respectively. Lines are model predictions based on original experimental conditions 

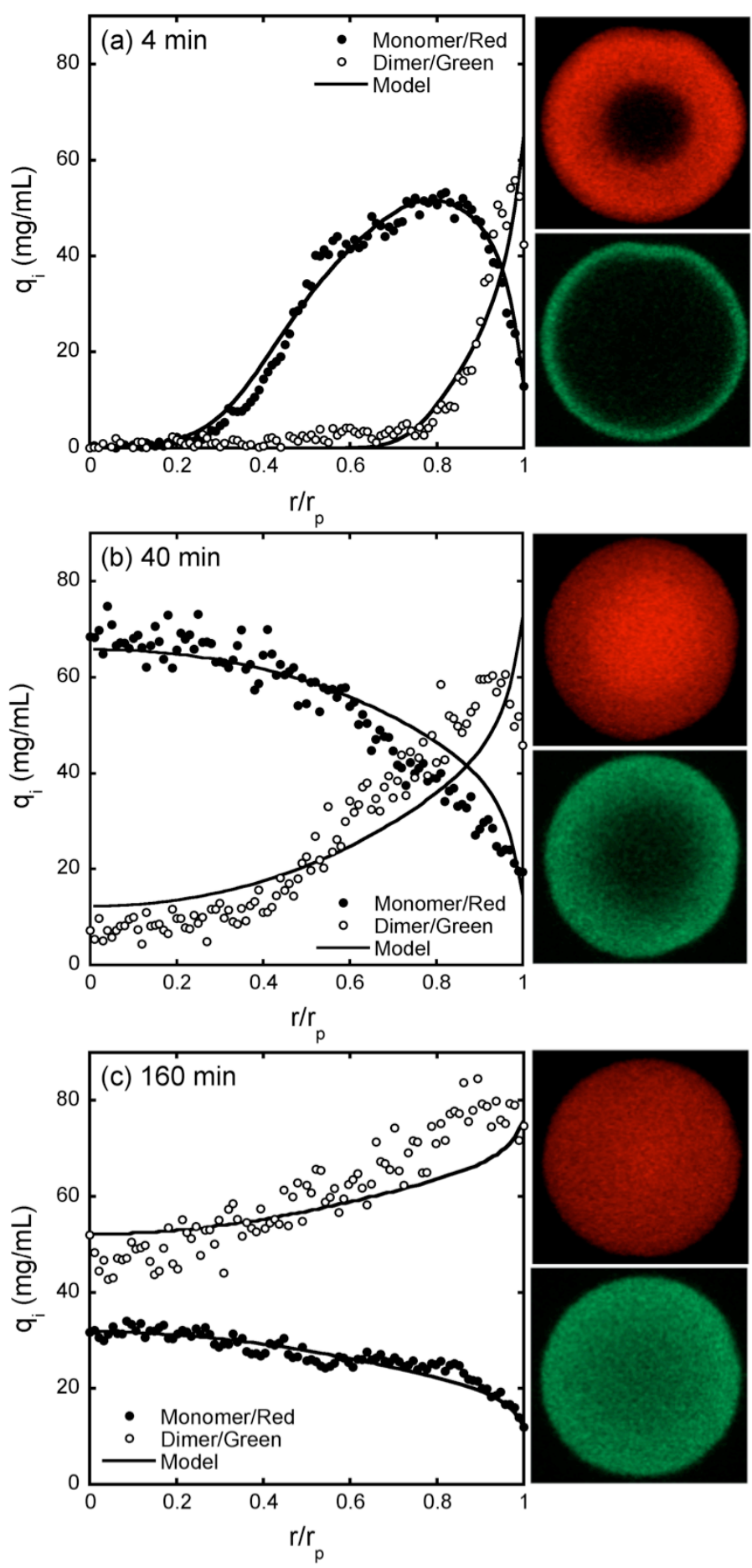

Figure 8.15. Comparison of experimental intraparticle concentration profiles of Fig. 7.8 with 80 $\mathrm{mM} \mathrm{Na}^{+}$with model predictions based on Eqs. 8.7 - 8.8. Solid and open circles are normalized fluorescence intensity profiles of the monomer and dimer, respectively. Lines are model predictions based on original experimental conditions 


\section{Kinetic Measurements via Biolayer Interferometry}

The results in chapters $7-8$ describing the two-component adsorption behavior of monoclonal antibody monomer and dimer on Nuvia HR-S showed behavior at low salt conditions that were inconsistent with traditional ion-exchange theory. Namely, the selectivity for higher valence molecules should be greater at these conditions. In modeling these results, a kinetic resistance to displacement was coupled to the standard pore diffusion model in an attempt to better capture the observed experimental trends. The experimental and modeling results are qualitatively consistent with a kinetic resistance that is strongest at low salt conditions and becomes less pronounced as salt concentration is increased. However, the porous structure of chromatographic media and large size of the protein molecules makes disentangling diffusional resistances from surface effects associated with adsorption and desorption a challenging task and prevents a mechanistic understanding of the actual displacement kinetics.

One potential solution to this problem is to directly measure the protein binding kinetics for conditions where mass transfer is fast using optical techniques such as surface plasmon resonance (SPR) (Lofas et al. 1991) or biolayer interferometry (Concepcion et al. 2009). The small volumes required and label-free nature of these measurements makes both techniques attractive; however, the disposable and high-throughput nature of BLI probes as well as the lack of microfluidics required for measurements offers BLI some additional advantages. BLI instruments monitor the temporal shift of light interference patterns generated from white light reflecting from an internal reference surface within a probe and from the functionalized surface of a biocompatible matrix at the distal tip of a probe (Chen 1998; Tan et al. 2008). These layers act as reflecting surfaces, which create an interference pattern, described by:

$$
I=I_{1}+I_{2}+2 \sqrt{I_{1} I_{2}} \cos \left(\frac{4 \pi n d}{\lambda}\right)
$$

where $I$ is the intensity of the wave, $n$ is the refractive index of the optical layer, $\lambda$ is the wavelength, and $d$ is the distance between the first reflecting surface and the second reflecting surface (Fig. 9.1a). Equation 9.1 has multiple maxima and minima that are continuously monitored by the system. As analytes adsorb and desorb to the functionalized 
surface, the interference pattern shifts due to changes in $d$ (Fig. 9.1b), allowing real-time visualization of adsorption and desorption kinetics. Figure 9.1c shows an example of how the intensity in Eq. 9.1 shifts as the length $d$ changes. Probes with surfaces functionalized with a variety of ligands, including reactive linkers, are commercially available.

BLI has already been used extensively for a number of different kinetic measurements. For example, Concepcion et al. (2009) demonstrated that BLI Protein A biosensors could quantitate crude antibody samples with accuracy similar to that obtained with Protein A HPLC columns. Yu et al. (2015) used BLI for epitope binning of crude IgG1 samples on a variety of functionalized sensors, including a custom biotinylated-anti-human Fc capture sensor. Do et al. (2008) used BLI with anti-human IgG Fc biosensors to quantitate the IgG dynamic binding capacity of various Protein A resins in packed columns. In each of these works, BLI was used to measure an affinity interaction between a particular ligand and a target analyte. However, other groups have used $\mathrm{BLI}$ for more novel measurements of difficult to quantify phenomena. For example, Naik et al. (2013) used BLI to quantify the kinetics of a pH-driven conformational change of anthrax protective antigen from a pre-pore to pore conformation as a function of $\mathrm{pH}$. Verzijl et al. (2017) captured human epidermoid carcinoma cells on BLI sensors and then correlated the change in signal to intracellular actin remodeling intitiated by exposing the cells to various agonistic compounds. These cases demonstrated the potential for BLI to help in quantifying systems that do not follow an affinity-driven ligand-analyte interaction. Finally, though not performed with BLI, Vicente et al. (2010a, 2010b) used an SPR chip functionalized with a weak anion ligand to analyze the adsorption mechanism of BSA as a function of salt concentration, and to extract isotherm and kinetic parameters, allowing for a consideration of ion-exchange phenomena divorced from mass transport resistances in conventional chromatographic media. However, this work considered only a single component system without consideration of whether additional resistances may be introduced by a multi-component system. 


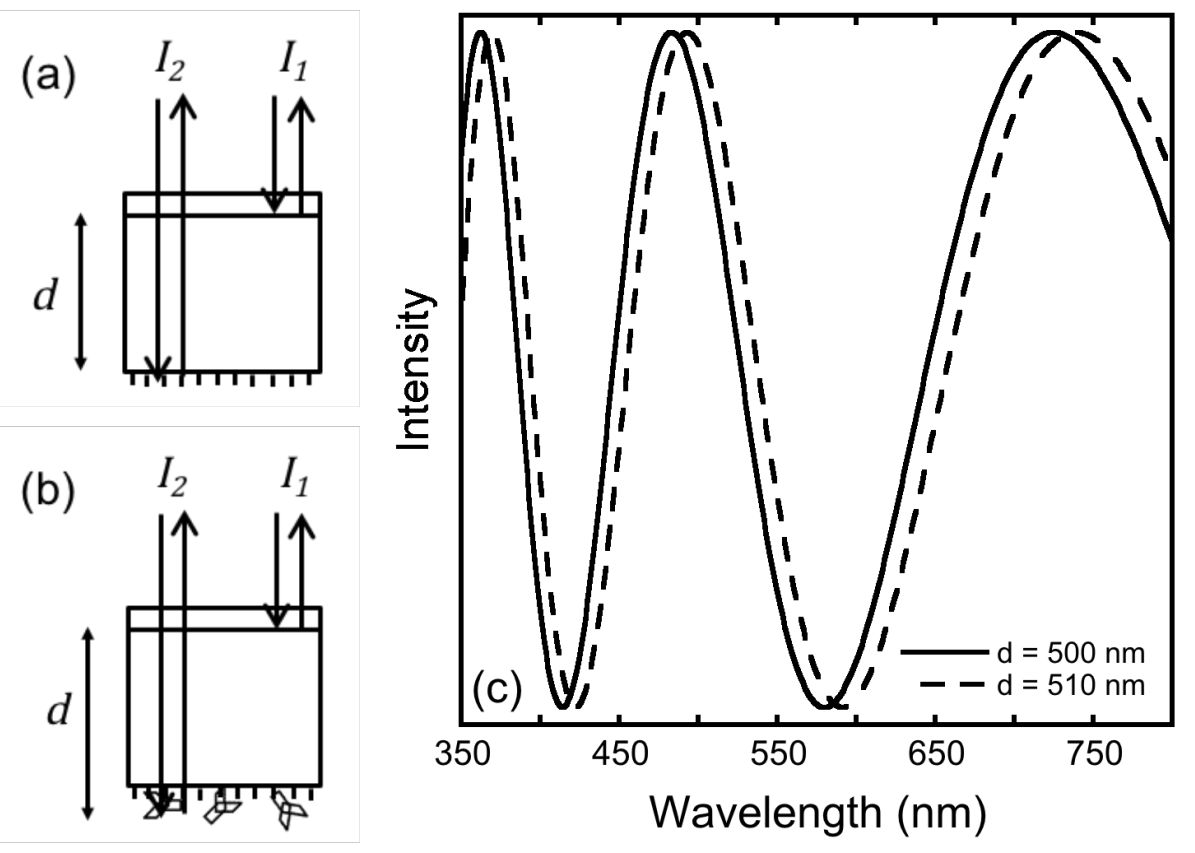

Figure 9.1. Schematic representation of a biolayer interferometry probe with (a) no species bound and (b) after binding. Intensity plot based on Eq. 9.1 before (solid) and after (dashed) binding an example species (c) 


\subsection{Probe functionalization attempts and characterization}

Attempts were made to functionalize the AR2G probe surfaces with a sulfopropyl ligand to match the functionality of Nuvia HR-S. For this purpose, the amine-terminated molecule 3amino-1-propanesulfonic acid (3-APS) was chosen. The AR2G reagent kit protocol from ForteBio uses an EDC (1-ethyl-3-[3-dimethylaminopropyl] carbodiimide) and s-NHS (Nhydroxysulfonsuccinimide) mixture to activate and stabilize the carboxyl groups to reactive NHS esters, which are then reacted with the primary amine on the ligand of interest (Pall ForteBio 2011a; Pall ForteBio 2011b). The reaction is quenched with an excess of ethanolamine to cap any remaining activated NHS esters. Following this protocol with 3-APS yielded BLI binding responses similar to those of unfunctionalized AR2G probes, albeit with a lower maximum binding response. Controls at low pH suggested no difference from the unfunctionalized probes, indicating the functionalization failed, possibly due to a lack of pre-concentration at the sensor surface due to the negatively charged sulfonic acid group of 3-APS. An altered protocol by Fischer (2010) for small, negatively charged molecules was attempted, but also failed to show a difference at low $\mathrm{pH}$ from the unfunctionalized commercial probes.

The difficulties in functionalizing the probe to a strong cation exchange surface led to continuing the work using the weak cation surface of the native AR2G probes at conditions where the surface should be fully deprotonated. Though the materials of the underlying matrix are not reported by ForteBio, most carboxylic acid groups have a $\mathrm{pK}_{\mathrm{a}}$ in the range of $2-5$ (Jinhua et al. 2006). To demonstrate the cationic nature of the probes, Fig. 9.2 shows AR2G sensorgrams of bovine serum albumin (BSA) binding in $10 \mathrm{mM} \mathrm{NaCH}_{3} \mathrm{COO}$ at pH 5.0 and $10 \mathrm{mM}$ $\mathrm{Na}_{2} \mathrm{HPO}_{4}$ at $\mathrm{pH} 7.0$ at three different protein concentrations. Due to its isoelectric point of 5.3, BSA will have a net positive charge at pH 5.0 and a net negative charge at pH 7.0. From Fig. 9.2, significant BSA binding is only observed at $\mathrm{pH} 5.0$, with the same concentrations of BSA at $\mathrm{pH}$ 7.0 exhibiting little to no binding, suggesting the AR2G probes are distinguishing based on the net BSA charge. A high salt wash at the end of the experiment desorbs a significant amount of the bound protein, though the signal does not return to baseline for the $\mathrm{pH} 5.0$ experiments. 

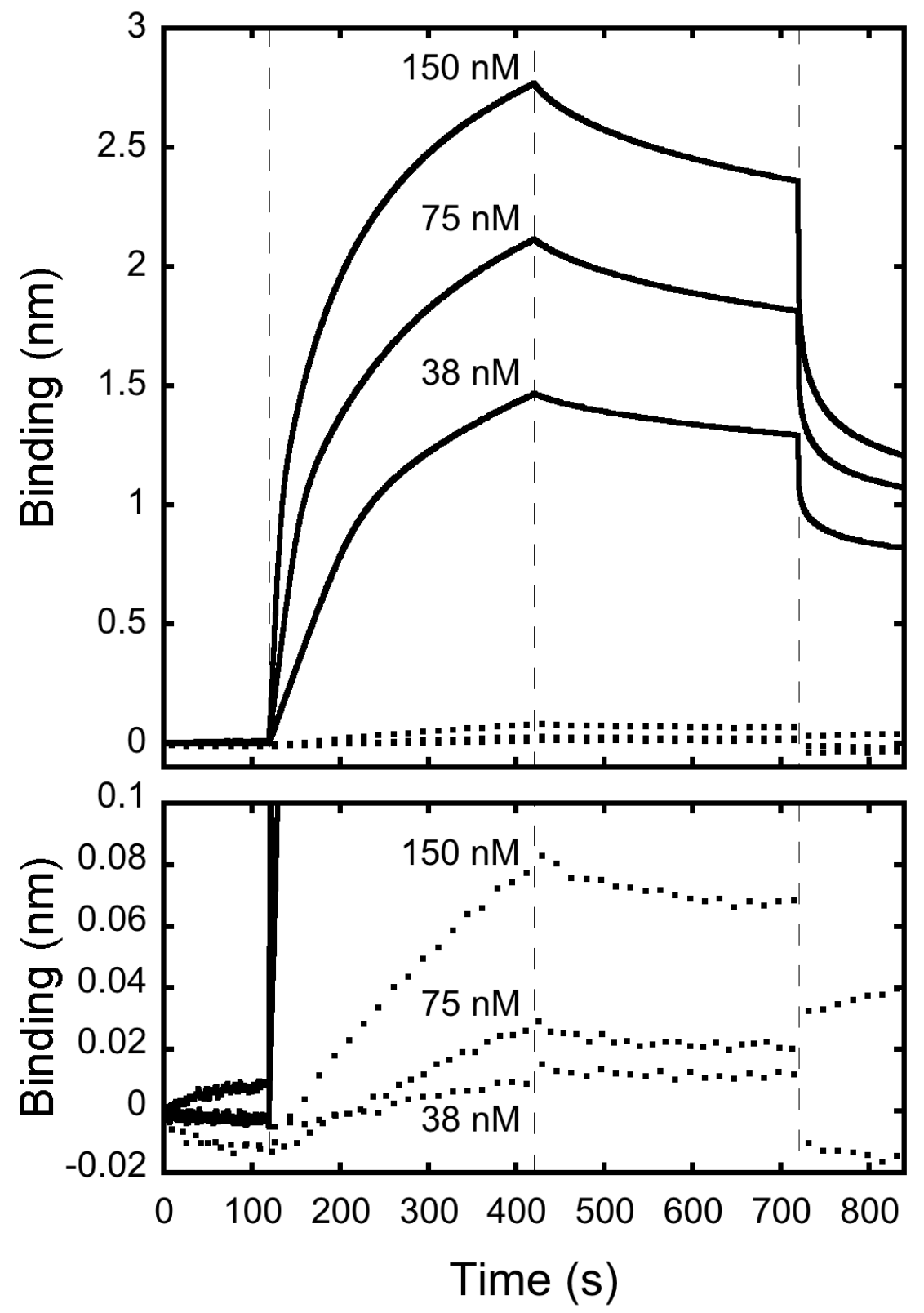

Figure 9.2. Sensorgrams of BSA binding on AR2 $\mathrm{G}$ probes in $20 \mathrm{mM} \mathrm{NaCH}{ }_{3} \mathrm{COO}$ at $\mathrm{pH} 5.0$ (solid) and in $10 \mathrm{mM} \mathrm{Na}_{2} \mathrm{HPO}_{4}$ at pH 7.0 (dotted and inset). Changes between phases are marked in dashed vertical lines 
This residual signal is likely due to irreversible binding of BSA to the sensor surface, possibly through hydrophobic interactions.

For further characterization, as a point of comparison, the porosity and pore radius results for Nuvia HR-S (Section 4.2) based on the single cylindrical pore model would yield a surface area of approximately $28 \mathrm{~m}^{2} / \mathrm{mL}$ particle. With the single component isotherm value for mAb monomer of $137 \mathrm{mg} / \mathrm{mL}$ particle (Section 6.2), this results in an approximate surface loading of $4.9 \mathrm{mg} / \mathrm{m}^{2}$. Biosensors from Pall ForteBio have a nominal tip diameter of $0.6 \mathrm{~mm}$. Additionally, they claim a biosensor surface can accommodate $10^{9}$ "capture sites" during protein linkage. Assuming a mAb monomer $\left(M_{r}=150,000\right)$ takes each of these sites, this results in an approximate surface loading of $0.88 \mathrm{mg} / \mathrm{m}^{2}$. These values are a similar order of magnitude, suggesting that the biosensors could serve as a reasonable approximation of a cation exchange surface.

A more rigorous interrogation of the tip protein binding capacity was attempted using fluorescently labeled protein. For these experiments, labeled mAb monomer was adsorbed to the tip surface and then desorbed into a $1 \mathrm{M} \mathrm{NaCl}$ solution. The protein concentration was then measured on a NanoDrop3300 Fluorospectrometer (Thermo Fisher Scientific, Waltham, MA, USA) using a fluorescence calibration curve. However, these measurements yielded unphysical measurements, with calculated loadings in excess of $100 \mathrm{mg} / \mathrm{m}^{2}$. The probe tips were then examined using an Eclipse E200 microscope with HB-10103AF Super High Pressure Mercury Lamp and Y-FL Epi-fluorescence attachments (Nikon Instruments Inc., Melville, NY, USA). Figure 9.3 shows microscope images of a probe tip after protein binding with visible light and under fluorescence. From Fig. 9.3b, it can be seen that binding is occurring along the length of the tip, in addition to the tip surface. This explains the unrealistic surface loadings, as the actual area of protein binding is significantly larger. However, without a way to differentiate between binding to the tip surface and binding to the tip length, a more rigorous and accurate measurement of the binding capacity of the tip surface is beyond the scope of this work. 


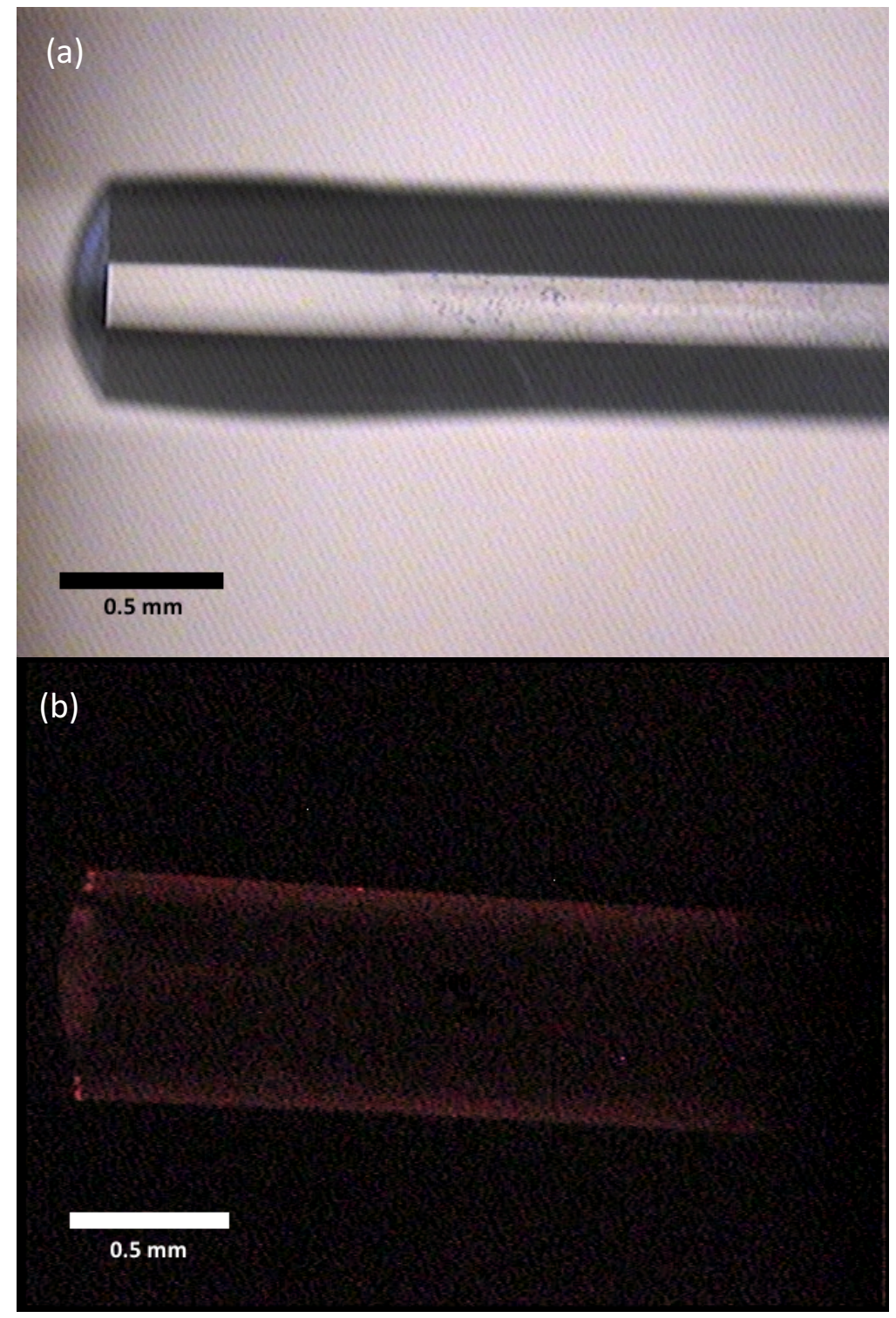

Figure 9.3. AR2G biosensor tip image at $4 \mathrm{x}$ magnification under (a) visible light and (b) fluorescence with bound mAb monomer 


\subsection{Single Component Measurements}

Figures 9.4 and 9.5 show the protein concentration dependent binding signals for single component monomer and single component dimer, respectively, at four different total $\mathrm{Na}^{+}$ concentrations. During the association phase, the highest protein concentration used gave a nearly vertical kinetic response in all cases, indicating immediate saturation of the probe and establishing the maximum binding signal at each salt concentration. The maximum signal achieved by the antibody dimer is higher than that of the monomer for all given salt concentrations, consistent with a higher equilibrium capacity of the dimer on the probe surface. Additionally, the difference between monomer and dimer binding decreases as the $\mathrm{Na}^{+}$ concentration decreases. This result is consistent with the results on Nuvia HR-S from Chapter 7, which showed little selectivity between monomer and dimer at low salt concentrations and higher selectivities at intermediate salt concentrations. The early time response during the association phase shows a proportional dependence on protein concentration at all $\mathrm{Na}^{+}$ concentrations measured. However, as $\mathrm{Na}^{+}$is increased, the maximum response obtained by a given protein concentration is reduced, indicating low binding and consistent with the behavior of ion-exchange resins. The dissociation phase of most of the experiments shows a biphasic response, with a rapid initial decrease in binding signal at early times followed by a much more gradual decay at longer times. This is most evident in the higher $\mathrm{Na}^{+}$experiments where binding is generally less favorable. For conditions where the binding is very favorable (low $\mathrm{Na}^{+}$ concentrations) but the probe is under-loaded during the association phase (low protein concentrations), the signal during dissociation is essentially flat, suggesting that dissociation is extremely slow for these conditions or that any dissociating protein is able to quickly re-adsorb. Following the dissociation phase, exposure to a high salt buffer results in an instantaneous desorption of nearly all of the bound protein, again consistent with the behavior of ionexchange resins. 

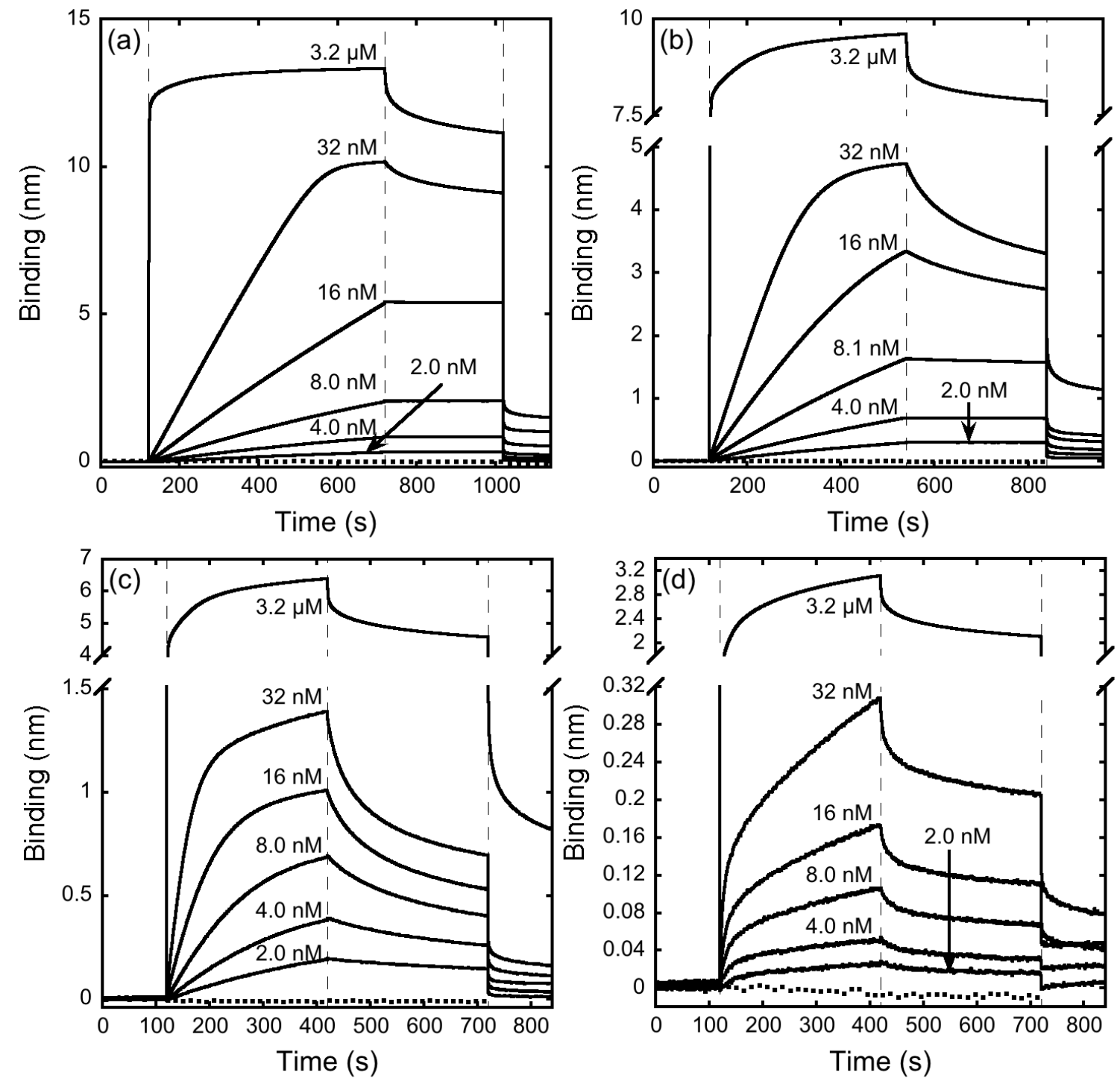

Figure 9.4. Sensorgrams for mAb monomer binding in $10 \mathrm{mM} \mathrm{Na}_{2} \mathrm{HPO}_{4}$ at $\mathrm{pH} 7.0$ with (a) $0 \mathrm{mM}$, (b) $30 \mathrm{mM}$, (c) $60 \mathrm{mM}$, and (d) $100 \mathrm{mM} \mathrm{NaCl}$. Reference sensor is shown as dotted line. Changes between phases are marked by dashed vertical lines 

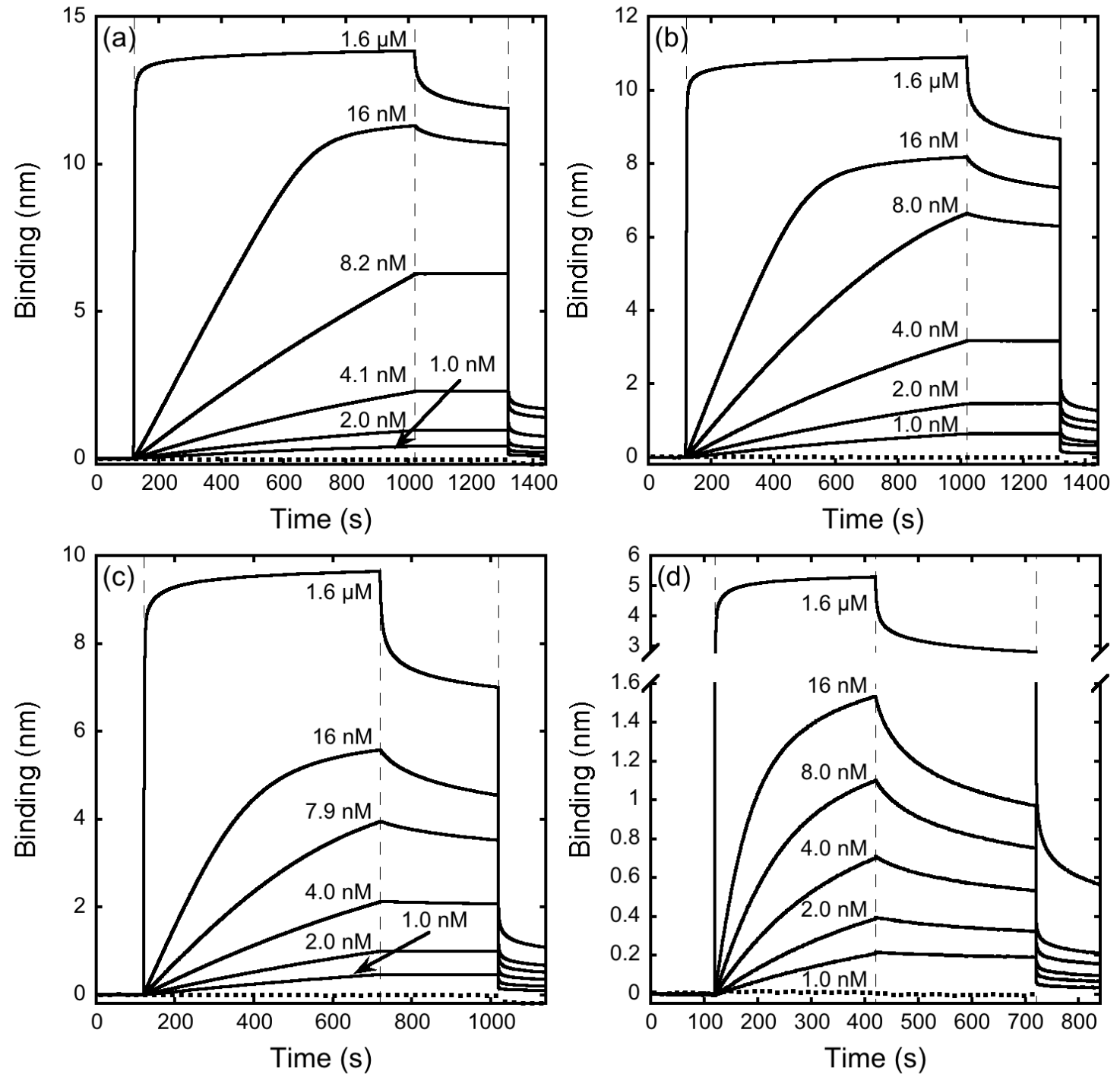

Figure 9.5. Sensorgrams for $\mathrm{mAb}$ dimer binding in $10 \mathrm{mM} \mathrm{Na}{ }_{2} \mathrm{HPO}_{4}$ at $\mathrm{pH} 7.0$ with (a) $0 \mathrm{mM}$, (b) $30 \mathrm{mM}$, (c) $60 \mathrm{mM}$, and (d) $100 \mathrm{mM} \mathrm{NaCl}$. Reference sensor is shown as dotted line. Changes between phases are marked by dashed vertical lines 


\subsection{Sequential Adsorption Measurements}

In these experiments, monomer and dimer were bound in various sequential manners in order to identify any kinetic limitations during exchange of the two molecules on the probe surface. Figure 9.6 shows the sequential adsorption of $32 \mathrm{nM}$ antibody monomer in both $20 \mathrm{mM} \mathrm{Na}^{+}$ (Fig. 9.6a) and $80 \mathrm{mM} \mathrm{Na}^{+}$(Fig. 9.6b), followed by the adsorption of the dimer (also in 20 or 80 $\mathrm{mM} \mathrm{Na}^{+}$) over the same range of protein concentrations shown in Figs. 9.5a and 9.5c. Each of these experiments utilized two reference probes, one with no protein during either the monomer or dimer association phase, and one with no protein during the dimer association phase. The monomer association and dissociation phases in Fig. 9.6 show excellent agreement for the different probes. The dimer association phase shows the impact that monomer binding and $\mathrm{Na}^{+}$concentration have on the subsequent dimer adsorption. The dimer association phase signals for the $20 \mathrm{mM} \mathrm{Na}^{+}$case (Fig. 9.6a) show only a modest further increase because of the high amounts of monomer already bound before exposure to the dimer. Conversely, the 80 $\mathrm{mM} \mathrm{Na}^{+}$results (Fig. 9.6b) show large increases in dimer signal because of the relatively low initial binding of monomer prior to exposure to the dimer. This result is consistent with the data in Chapter 7, showing stronger binding of the dimer compared to the monomer at this $\mathrm{Na}^{+}$ concentration on Nuvia HR-S.

Figure 9.7 shows the sequential adsorption of $17 \mathrm{nM}$ antibody dimer in both $20 \mathrm{mM} \mathrm{Na}^{+}$(Fig. 9.7a) and $80 \mathrm{mM} \mathrm{Na}^{+}$(Fig. 9.7b), followed by the adsorption of monomer (also in 20 or $80 \mathrm{mM}$ $\mathrm{Na}^{+}$), which is the reverse of the experiment in Fig. 9.6. Similar to Fig. 9.6, the dimer association and dissociation phases show good reproducibility from probe to probe, and are in good agreement with the response seen for the $16 \mathrm{nM}$ dimer runs in Figs. 9.5a and 9.5c. As with Fig. 9.6a, the second association phase of monomer shows very little additional signal increase, due to the large amounts of dimer already bound. In Fig. 9.7b, aside from the very high concentration sample, essentially no monomer association occurs, because in these instances the surface binding already exceeds what would be expected for monomer in $80 \mathrm{mM} \mathrm{Na}^{+}$. Figures 9.6 and 9.7 show the adsorption response in the second association phase is strongly linked to the binding during the first association phase. 

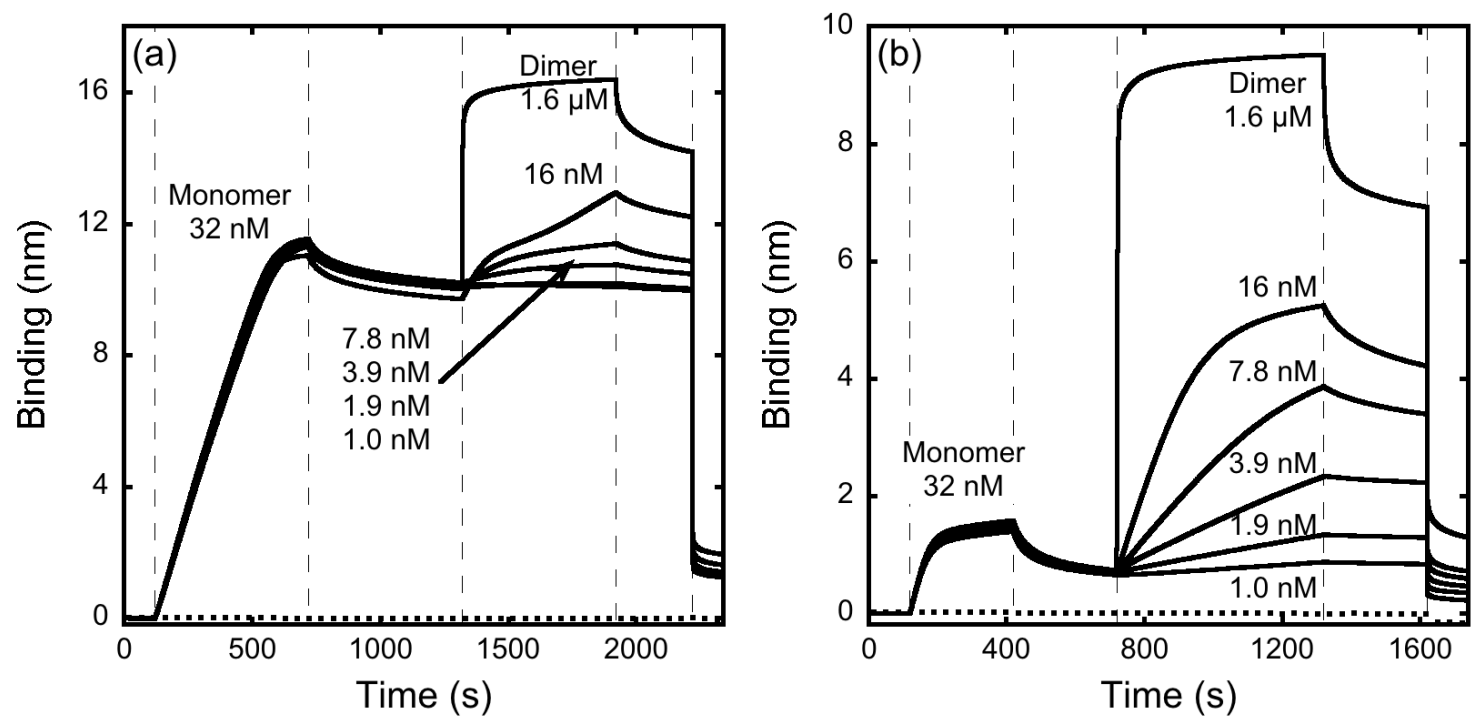

Figure 9.6. Sensorgrams for sequential binding of $32 \mathrm{nM}$ mAb monomer followed by varying concentrations of mAb dimer in $10 \mathrm{mM} \mathrm{Na}_{2} \mathrm{HPO}_{4}$ at pH 7.0 with (a) $0 \mathrm{mM}$ and (b) $60 \mathrm{mM} \mathrm{NaCl}$. Reference sensors are shown as dotted lines. Changes between phases are marked by dashed vertical lines 

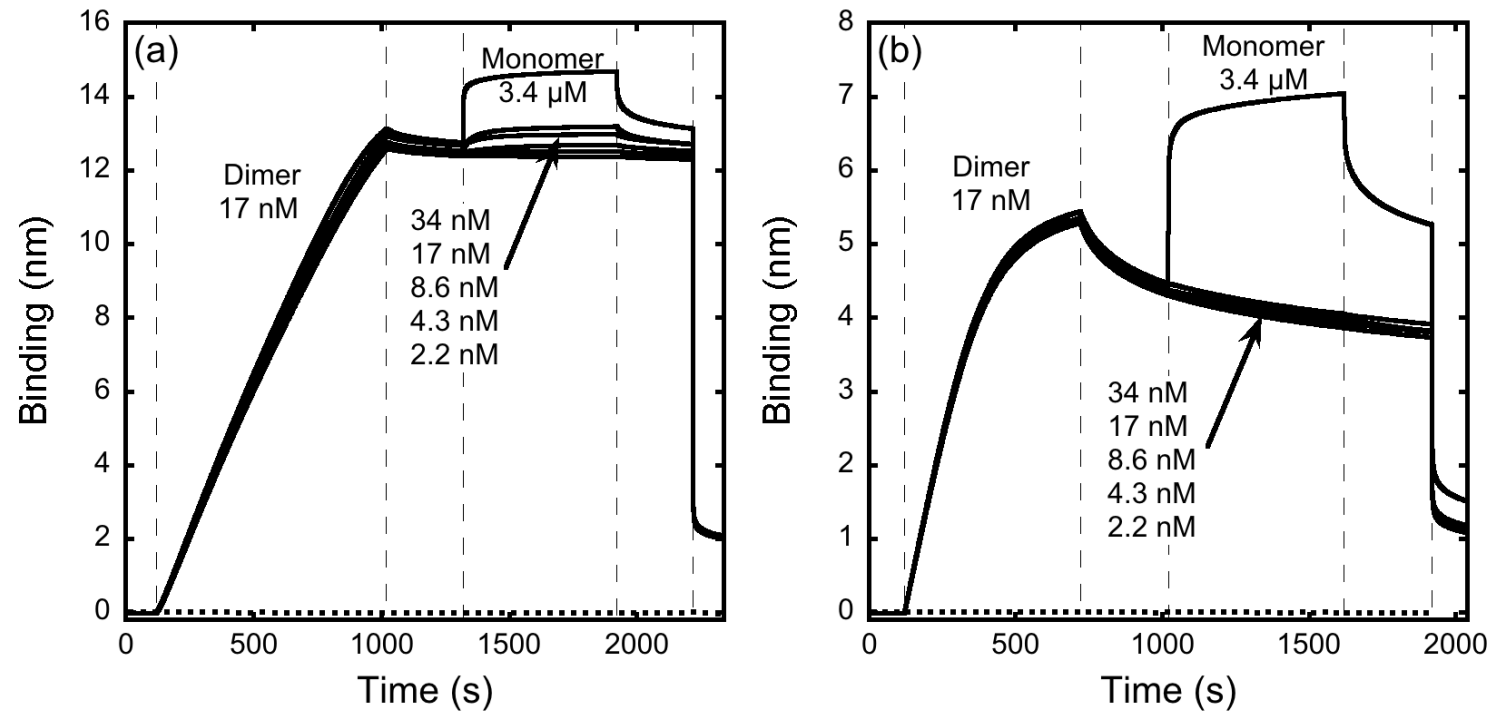

Figure 9.7. Sensorgrams for sequential binding of $17 \mathrm{nM}$ mAb dimer followed by varying concentrations of mAb monomer in $10 \mathrm{mM} \mathrm{Na}_{2} \mathrm{HPO}_{4}$ at pH 7.0 with (a) $0 \mathrm{mM}$ and (b) $60 \mathrm{mM}$ $\mathrm{NaCl}$. Reference sensors are shown as dotted lines. Changes between phases are marked by dashed vertical lines 
In order to control the amount of protein bound during the first association phase, mixed buffer experiments were performed where $34 \mathrm{nM}$ antibody monomer was bound at different $\mathrm{Na}^{+}$ concentrations $(20,30,50$, and $80 \mathrm{mM})$. This was followed by a dissociation phase in $20 \mathrm{mM}$ $\mathrm{Na}^{+}$, which results in minimal monomer dissociation, essentially 'freezing' the bound monomer to the probe surface. Accordingly, this allows measurement of the kinetics of either monomer or dimer binding to surfaces that contain different amounts of bound monomer for otherwise identical conditions. Figure $9.8 \mathrm{a}$ and $9.8 \mathrm{~b}$ show the results of these experiments. In the first case, shown in Fig. 9.8a, the second association phase contains $34 \mathrm{nM}$ antibody monomer in 20 $\mathrm{mM} \mathrm{Na}^{+}$, creating essentially a single component experiment with discontinuous loading. As seen from this figure, varying the $\mathrm{Na}^{+}$concentration in the first association and dissociation phases has the intended effect of changing the initial protein bound prior to the second association. During the second association phase, the sensorgram signals increase by different amounts dependent on the initial bound monomer, before reaching the same final value. In the second case, shown in Fig. 9.8b, the second association phase contains $17 \mathrm{nM}$ antibody dimer in $20 \mathrm{mM} \mathrm{Na}^{+}$. The behavior is obviously different from that shown in Fig. 9.8a. While all of the second association phases signals reach the same final value, the shapes of the curves differ dependent on the initially bound monomer. For the sensorgrams where high amounts of monomer were initially bound (e.g. in 20 and $30 \mathrm{mM} \mathrm{Na}^{+}$), the second association phase shows multiple inflection points, the first of which occurs near the maximum binding signal for monomer, followed by a slow increase in signal before reaching the final value. Sensorgrams corresponding to lower initial monomer binding (e.g. in 50 and $80 \mathrm{mM} \mathrm{Na}^{+}$) exhibit instead curve shapes similar to those in Fig. 9.8a. The slow rate observed during the dimer second association for high amounts of initially bound monomer indicates that a significant kinetic resistance exists during the displacement of the already bound monomer by the dimer.

\subsection{Modeling and Parameter Estimation}

Various models could be used to represent binding to surfaces. However, since the goal is to correlate the data so they can be used to predict kinetic effects in protein chromatography 

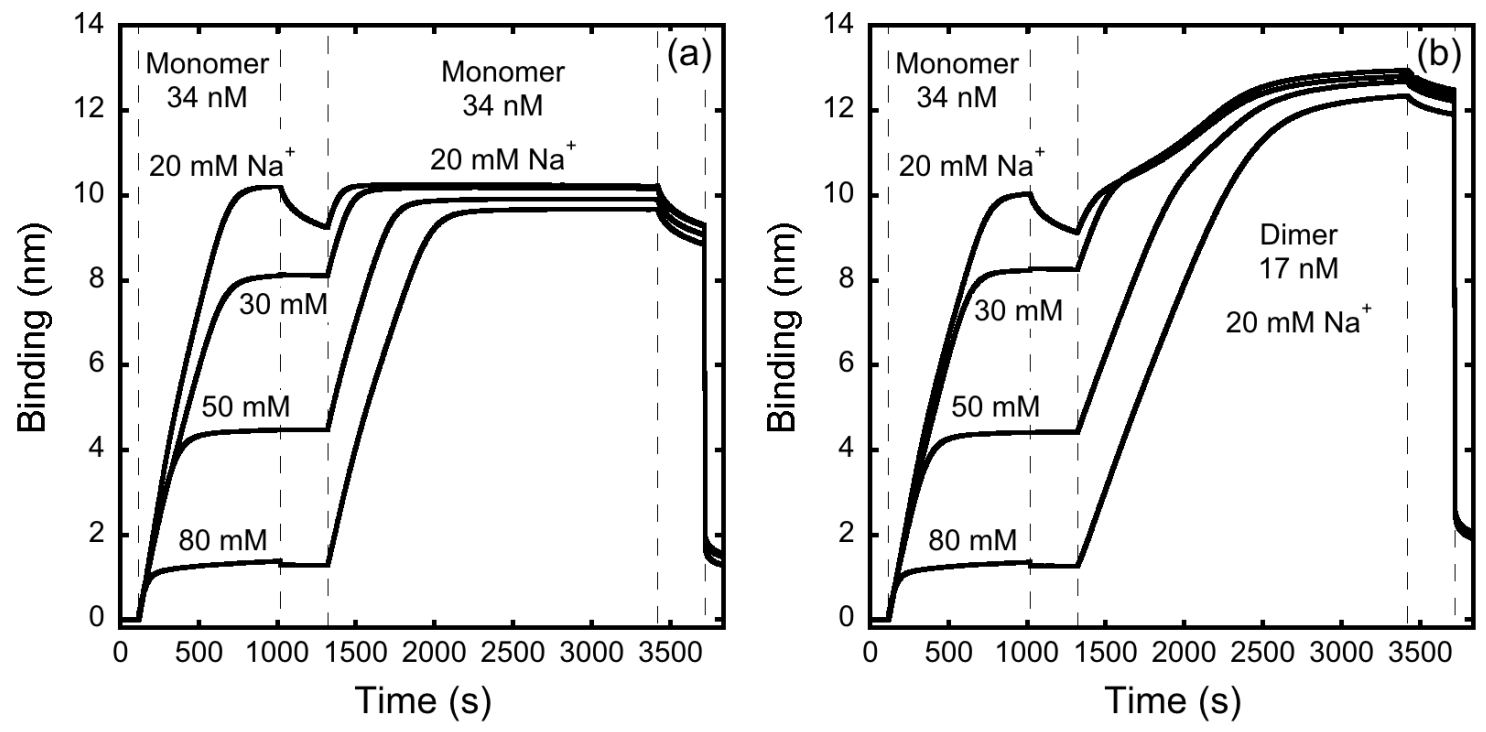

Figure 9.8. Sensorgrams for sequential binding of $34 \mathrm{nM} \mathrm{mAb}$ monomer in $10 \mathrm{mM} \mathrm{Na}_{2} \mathrm{HPO}_{4}$ at pH 7.0 with $0,10,30$, and $60 \mathrm{mM} \mathrm{NaCl}$ followed by (a) $34 \mathrm{nM}$ mAb monomer or (b) $17 \mathrm{nM} \mathrm{mAb}$ dimer in $10 \mathrm{mM} \mathrm{Na}_{2} \mathrm{HPO}_{4}$ at pH 7.0 with $0 \mathrm{mM} \mathrm{NaCl}$. Changes between phases are marked by dashed vertical lines 
columns, only simplified rate equations were considered. The simplest such model assumes a 1:1 interaction, with a single surface site binding a single protein molecule (O'Shannessy et al. 1993; O’Shannessy 1994; Önell \& Andersson 2005; Schuck 1997). Assuming no change in the protein concentration during the association and dissociation phases, the integrated rate expression for one component binding and dissociation from the surface is given by:

$$
\begin{aligned}
& R_{\text {assoc }}(t)=\frac{R_{\max } k_{a} C}{k_{a} C+k_{d}}\left\{1-\exp \left[-\left(k_{a} C+k_{d}\right) t\right]\right\} \\
& R_{\text {dissoc }}(t)=-R_{\text {assoc }}\left(t_{0}\right) \exp \left\{-\left[k_{d}\left(t-t_{0}\right)\right]\right\}
\end{aligned}
$$

where $R_{\max }$ is the maximum binding signal, $C$ is the protein concentration, $k_{a}$ is the association rate constant, $k_{d}$ is the dissociation constant, and $t_{0}$ is the time at the start of the dissociation phase. Assuming that protein binding to the surface and the BLI signal are linearly related, $R_{\max }$ is proportional to the maximum protein binding. The model is particularly applicable for fitting affinity interactions where a 1:1 interaction is more likely to be a valid assumption. However, in the experiments performed in this work, it is immediately obvious that a 1:1 model will fail to capture certain behaviors, such as the biphasic dissociation behavior. When encountering biphasic behavior, O'Shannessey et al. (1993) recommended a two-site model, which assumes two independent interactions accounting for the binding signal. The integrated rate expressions for the association and dissociation phases in a two-site model are given by:

$$
\begin{aligned}
& R_{\text {assoc }}(t)=\frac{R_{\text {max }, 1} k_{a, 1} C}{k_{a, 1} C+k_{d, 1}}\left\{1-\exp \left[-\left(k_{a, 1} C+k_{d, 1}\right) t\right]\right\}+\frac{R_{\max , 2} k_{a, 2} C}{k_{a, 2} C+k_{d, 2}}\left\{1-\exp \left[-\left(k_{a, 2} C+k_{d, 2}\right) t\right]\right\} \\
& R_{\text {dissoc }}(t)=R_{a s s o c, 1}\left(t_{0}\right) \exp \left\{-\left[k_{d, 1}\left(t-t_{0}\right)\right]\right\}+R_{a s s o c, 2}\left(t_{0}\right) \exp \left\{-\left[k_{d, 2}\left(t-t_{0}\right)\right]\right\}
\end{aligned}
$$

where $R_{\max }, C, k_{a}$, and $k_{d}$ are the same as defined in Eq. 9.2, with the subscripts 1 and 2 denoting the two different sites, and $R_{a s s o c .1}$ and $R_{a s s o c, 2}$ refer to the $1^{\text {st }}$ and $2^{\text {nd }}$ term, respectively, in Eq. 9.3a.

Additional models were also examined for fitting. Edwards et al. (1998) proposed a secondorder model accounting for depletion during the association phase, and accumulation during 
the dissociation phase. However, reference probes during the sequential adsorption experiments underwent dissociation in multiple wells, and showed no increase in dissociation rate upon entering a fresh well, suggesting accumulation of dissociated protein was not responsible for the biphasic dissociation behavior (see Fig. 9.7b). The steric mass-action (SMA) model where $\mathrm{Na}^{+}$concentration is explicitly accounted for was also examined, but due to the global nature of the fitting, resulted in constrained fits that showed good agreement for certain $\mathrm{Na}^{+}$concentrations, but not others (Brooks \& Cramer 1992). Finally, Johnson and Arnold suggested that heterogeneous binding (as may be expected in ion-exchange) could be described by a Temkin isotherm model, where site energies decrease linearly with surface coverage (Johnson \& Arnold 1995b; Johnson \& Arnold 1995a). Fits to this model form were not appreciably better than the one-site model and were significantly worse than the two-site model. Sensorgram modeling for all of the models was done in MATLAB R2015b (The Mathworks, Natick, MA, USA). For models with an integrate rate equation, the Isqnonlin nonlinear least squares solver was used to solve for the best-fit parameters. For models without an analytical time-dependent solution, the ode15s variable order differential equation solver was used in conjunction with Isqnonlin to find the best-fit parameters. Parameter confidence intervals were determined using nlparci.

Figures 9.9 and 9.10 show model fits for the one-site and two-site models for the antibody monomer and dimer experiments from Figs. 9.4 and 9.5. $R_{\max }$ values for both the one-site and two-site models were fitted for each individual protein concentration. For the two-site model fitting, the $R_{\max }$ associated with the second site averaged $10.5 \%$ of the total $R_{\max }$. Following previously established best practices (O'Shannessy et al. 1993; Edwards et al. 1998), the dissociation curves were analyzed independently to obtain unambiguous dissociation constants, which were then constrained during global fitting of the remaining parameters. Table 9.1 summarizes the association and dissociation constants of the two models for both monomer and dimer at each individual salt concentration. As discussed above, Figs. 9.9 and 9.10 show the one-site binding model is unable to capture the biphasic dissociation data, whereas the two-site model is able to capture the behavior quite well. For the dissociation 

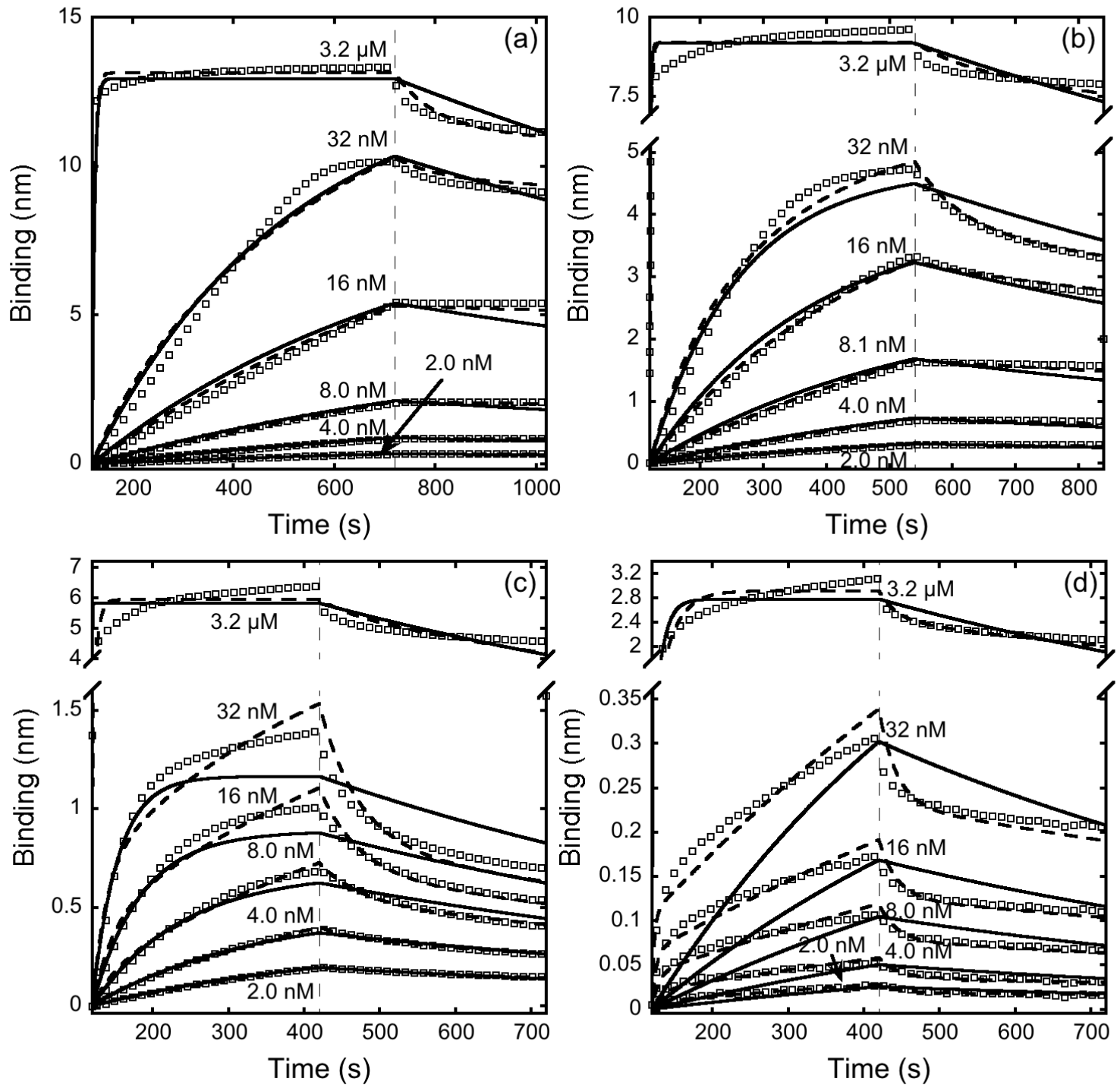

Figure 9.9. Experimental data (open squares) and model fits for a one-site (solid line) and twosite (dashed line) binding model for antibody monomer in different concentrations of total $\mathrm{Na}^{+}$. Experimental conditions are the same as in Fig. 9.4. One-site model based on Eq. 9.2; two-site model based on Eq. 9.3 with parameters for both Eqs. given in Table 9.1 

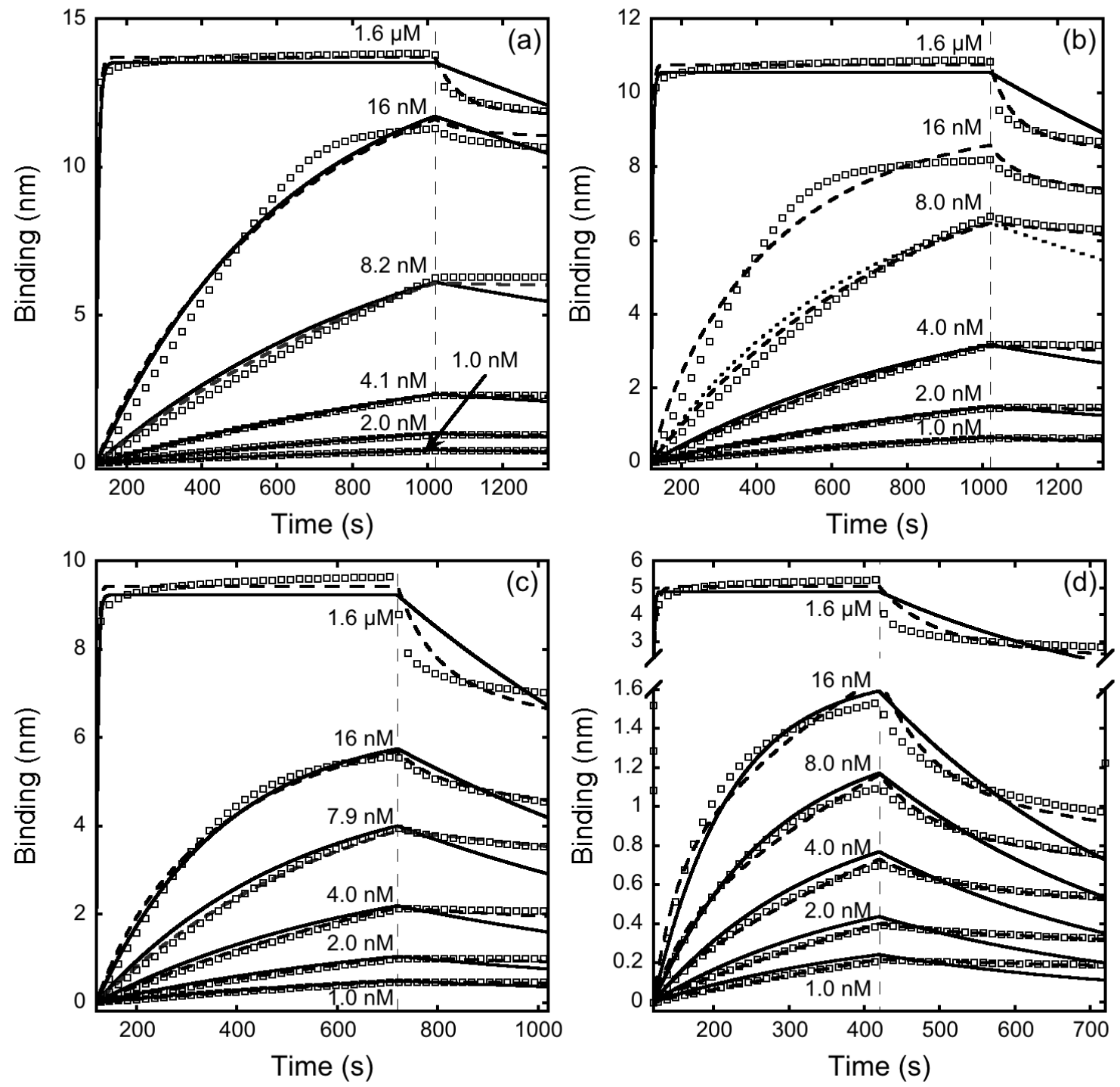

Figure 9.10. Experimental data (open squares) and model fits for a one-site (solid line) and twosite (dashed line) binding model for antibody dimer in different concentrations of total $\mathrm{Na}^{+}$. Experimental conditions are the same as in Fig. 9.5. One-site model based on Eq. 9.2; two-site model based on Eq. 9.3 with parameters for both Eqs. given in Table 9.1 


\begin{tabular}{|c|c|c|c|c|c|c|c|}
\hline \multirow[b]{2}{*}{ Protein } & \multirow[b]{2}{*}{$\begin{array}{l}\mathrm{C}_{\mathrm{Na}^{+}} \\
(\mathrm{mM})\end{array}$} & \multicolumn{2}{|c|}{ 1:1 Binding Model } & \multicolumn{2}{|c|}{$\begin{array}{l}\text { 2:1 Binding Model } \\
\text { Site } 1 \text { (Strong Site) }\end{array}$} & \multicolumn{2}{|c|}{$\begin{array}{c}\text { 2:1 Binding Model Site } \\
2 \text { (Weak Site) }\end{array}$} \\
\hline & & $\begin{array}{c}k_{a}(\mathrm{a}) \\
\left(10^{4} \mathrm{M}^{-1} \mathrm{~s}^{-1}\right)\end{array}$ & $\begin{array}{c}k_{d}(\mathrm{a}) \\
\left(10^{-4} \mathrm{~s}^{-1}\right)\end{array}$ & $\begin{array}{c}k_{a, 1} \text { (a) } \\
\left(10^{4} \mathrm{M}^{-1} \mathrm{~s}^{-1}\right)\end{array}$ & $\begin{array}{c}k_{d, 1}(\mathrm{~b}) \\
\left(10^{-4} \mathrm{~s}^{-1}\right)\end{array}$ & $\begin{array}{c}k_{a, 2} \text { (a) } \\
\left(10^{4} \mathrm{M}^{-1} \mathrm{~s}^{-1}\right)\end{array}$ & $\begin{array}{c}k_{d, 2}(\mathrm{~b}) \\
\left(10^{-2} \mathrm{~s}^{-1}\right)\end{array}$ \\
\hline \multirow{4}{*}{ Monomer } & 20 & $6.44 \pm 0.08$ & $\begin{array}{l}5.11 \pm \\
0.07\end{array}$ & $6.53 \pm 0.10$ & $\begin{array}{l}0.92 \pm \\
0.58\end{array}$ & $19.8 \pm 3.6$ & $\begin{array}{l}1.25 \pm \\
0.35\end{array}$ \\
\hline & 50 & $19.5 \pm 0.2$ & $\begin{array}{l}7.56 \pm \\
0.08\end{array}$ & $13.0 \pm 0.3$ & $\begin{array}{c}3.83 \pm \\
1.85\end{array}$ & $12.0 \pm 2.8$ & $\begin{array}{c}1.32 \pm \\
0.49\end{array}$ \\
\hline & 80 & $83.8 \pm 2.9$ & $\begin{array}{c}11.5 \pm \\
0.2\end{array}$ & $5.79 \pm 0.12$ & $\begin{array}{c}9.03 \pm \\
2.87\end{array}$ & $109 \pm 13.6$ & $\begin{array}{c}1.98 \pm \\
0.70\end{array}$ \\
\hline & 120 & $2.62 \pm 0.04$ & $\begin{array}{c}12.5 \pm \\
0.2\end{array}$ & $1.38 \pm 0.02$ & $\begin{array}{l}6.63 \pm \\
2.12\end{array}$ & $892 \pm 444$ & $\begin{array}{l}4.41 \pm \\
0.70\end{array}$ \\
\hline \multirow{4}{*}{ Dimer } & 20 & $9.81 \pm 0.09$ & $\begin{array}{c}3.77 \pm \\
0.06\end{array}$ & $11.0 \pm 0.1$ & $\begin{array}{c}0.38 \pm \\
0.23\end{array}$ & $44.5 \pm 9.5$ & $\begin{array}{c}1.63 \pm \\
0.44\end{array}$ \\
\hline & 50 & $16.6 \pm 0.2$ & $\begin{array}{l}5.65 \pm \\
0.07\end{array}$ & $17.1 \pm 0.1$ & $\begin{array}{l}1.63 \pm \\
0.65\end{array}$ & $23.0 \pm 2.8$ & $\begin{array}{l}1.75 \pm \\
0.41\end{array}$ \\
\hline & 80 & $18.5 \pm 0.3$ & $\begin{array}{c}10.6 \pm \\
0.1\end{array}$ & $20.7 \pm 0.3$ & $\begin{array}{c}3.20 \pm \\
0.43\end{array}$ & $25.7 \pm 2.4$ & $\begin{array}{l}1.43 \pm \\
0.25\end{array}$ \\
\hline & 120 & $45.6 \pm 2.4$ & $\begin{array}{c}26.2 \pm \\
0.2\end{array}$ & $14.4 \pm 0.5$ & $\begin{array}{c}4.86 \pm \\
2.14\end{array}$ & $147 \pm 13.9$ & $\begin{array}{c}1.42 \pm \\
0.55\end{array}$ \\
\hline
\end{tabular}

Table 9.1. Kinetic parameters from one-site and two-site binding models for monomer and dimer at varying salt concentrations
(a) Error is $95 \%$ confidence interval of non-linear regressed parameter
(b) Error is standard deviation of parameter value calculated from individual dissociation phases 
constants of the two-site model listed in Table 9.1, it appears that the second (weaker) site demonstrates a fairly constant value across both proteins and at all salt concentrations studied, which is responsible for the quick initial dissociation in the experiments while the first (stronger) site demonstrates a slight increase as the $\mathrm{Na}^{+}$concentration is increased, indicating weaker overall binding. Additionally, this stronger site dissociation constant is lower for the dimer than for the monomer at all salt concentrations observed, indicative of the overall stronger binding of the dimer to the surface.

Both the one-site and two-site models show similar fits for the association phase data, and neither is accurately able to capture the entirety of the behavior during association. This is more apparent if the association data is transformed to a binding rate vs. binding plot, which has also been used previously to extract kinetic parameters as an alternative to non-linear regression techniques (O’Shannessy 1994; Karlsson et al. 1991; Zeder-Lutz et al. 1993; Jendeberg et al. 1995). Figures 9.11 and 9.12 show the association phase binding rate averaged over $10 \mathrm{~s}$ intervals vs. total binding for the antibody monomer and dimer experiments depicted in Figs. 9.4 and 9.5, respectively. Data well described by a one-site model would show rates that linearly decrease to zero as the maximum binding signal is approached. However, these rate plots show two different rate regimes during the binding process. The first occurs at low total binding, far away from the maximum binding signal (apparent in Figs. 9.11a-b and 9.12a-b), and the second occurs as the binding response approaches the maximum binding signal, at which point the slope changes as the rate drops to zero. For conditions where the maximum binding signal is already very low (e.g. $120 \mathrm{mM} \mathrm{Na}^{+}$), the rate appears to be dominated by the second regime. Somewhat counter intuitively, the initial rate of association (at low loadings) seems to be independent of $\mathrm{Na}^{+}$concentration. This is also apparent during the first association phases in Fig. 9.8, where the differing $\mathrm{Na}^{+}$concentrations only appear to impact the maximum binding signal.

This rate analysis is also useful in visualizing the kinetic resistance due to the pre-bound monomer observed in the sequential protein binding experiments of Fig. 9.8. Figure 9.13 shows 
the binding rate averaged over $10 \mathrm{~s}$ intervals vs. protein binding for these experiments. For Fig. 9.13a, as with Figs. 9.11 and 9.12, the rate curves all have the same general shape with two distinct rate regimes, one faster regime at lower loadings, and one much slower regime as the maximum binding signal is approached. However, a different behavior is obvious from Fig. 9.13b where the dimer binds to a surface that is preloaded with different amounts of monomer. For experiments where high amounts of monomer were initially bound (e.g. in 20 and $30 \mathrm{mM} \mathrm{Na}^{+}$), the rate decreases precipitously as it reaches the signal corresponding to maximum monomer binding. Binding then continues at a much lower rate until the signal corresponding to maximum dimer binding is reached. For experiments where lower amounts of monomer were initially bound (e.g. in 50 and $80 \mathrm{mM} \mathrm{Na}^{+}$), there is no decrease at the maximum monomer binding value, and the rate decreases monotonically much like the monomer curves in Fig. 9.13a.

The complexity of these association curves highlight the limitations of the relatively simple models proposed. Figure 9.14 shows the two-site model fit of the 20 and $80 \mathrm{mM} \mathrm{Na}^{+}$sequential monomer and dimer experiments shown in Fig. 9.8b using the kinetic parameters from the single component experiments. Because the initial condition is not zero for the second association phase, a differential form of the two-site model was used:

$$
\frac{d R_{i, n}}{d t}=k_{a i, n} C_{i}\left(R_{\max i, n}-\sum_{j} R_{j, n}\right)-k_{d i, n} R_{i, n}
$$

where $R_{\max }, C, k_{a}$, and $k_{d}$ are the same as defined in Eq. 9.2, with the subscripts $i$ denoting the species and $n$ denoting the binding site. This allows for a decoupling of the monomer and dimer signals to show the impact the monomer preloading has on the subsequent dimer association. Figure 9.14a shows the slow dissociation of the monomer, which prevents the dimer from quickly binding to the surface. The model is obviously unable to capture the inflection points in the second association. Figure $9.14 \mathrm{~b}$ shows that because of the reduced preloading of the monomer, the dimer is readily able to bind to the surface in a manner very similar to the single component experiments. 


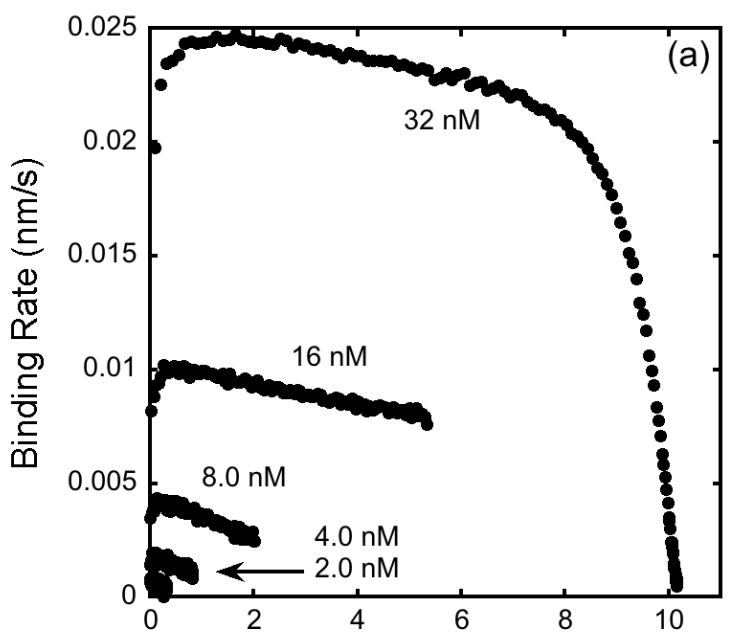

Binding ( $\mathrm{nm})$

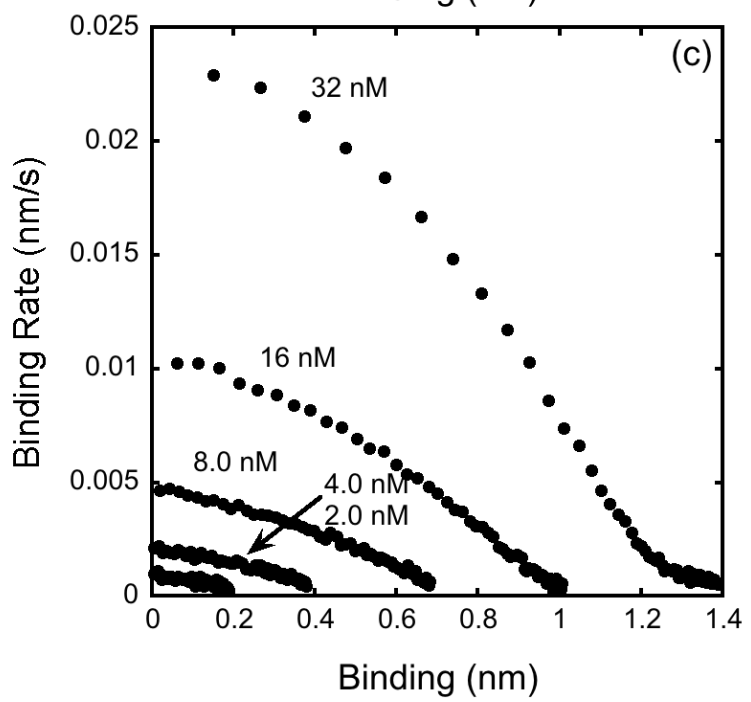

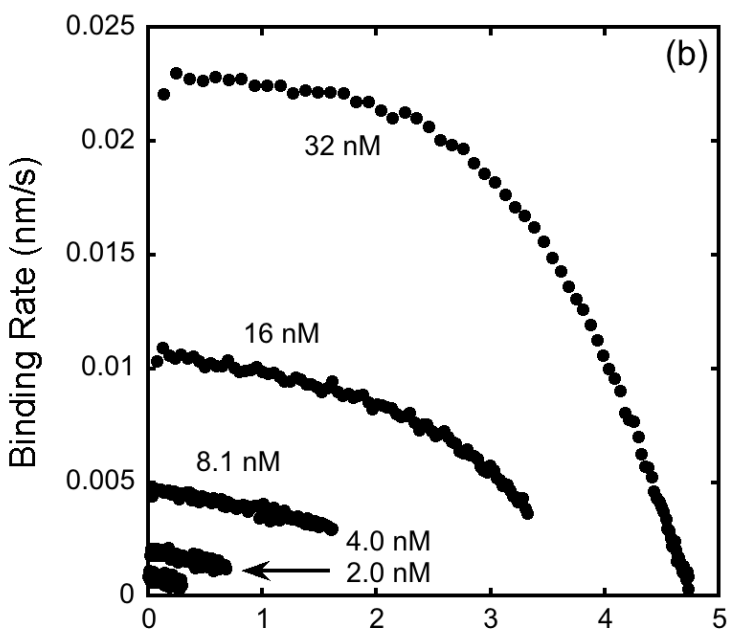

Binding ( $\mathrm{nm})$

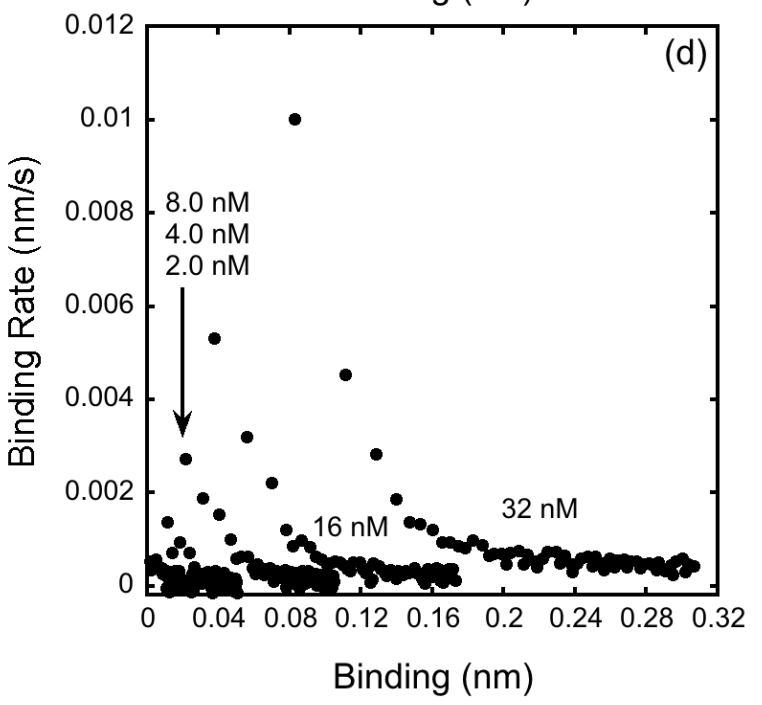

Figure 9.11. Binding rate during association phase of monomer experiments depicted in Fig 9.4 vs. total probe binding. Experimental conditions are the same as described in Fig. 9.4 

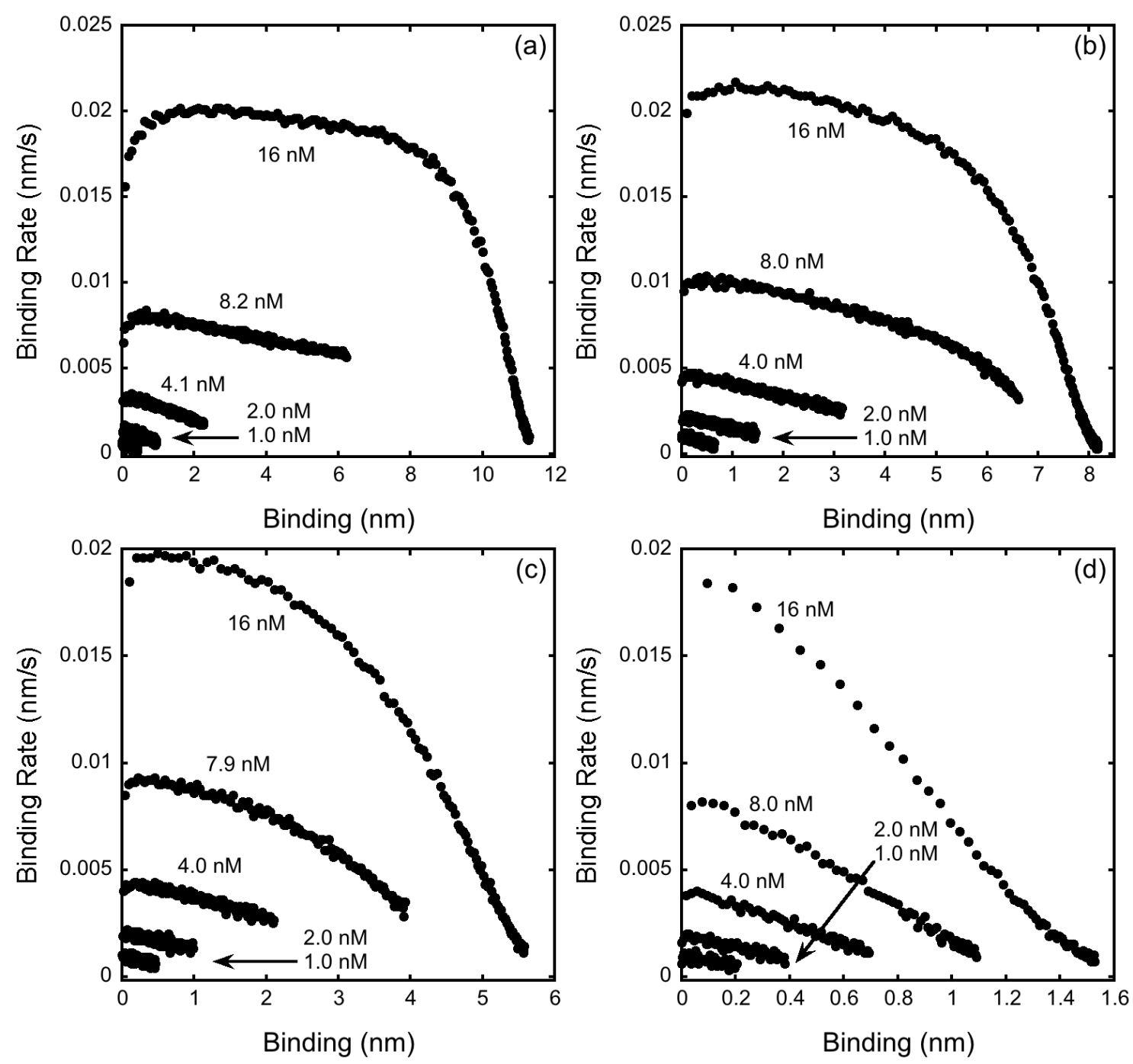

Figure 9.12. Binding rate during association phase of dimer experiments depicted in Fig. 9.5 vs. total probe binding. Experimental conditions are the same as described in Fig. 9.5 

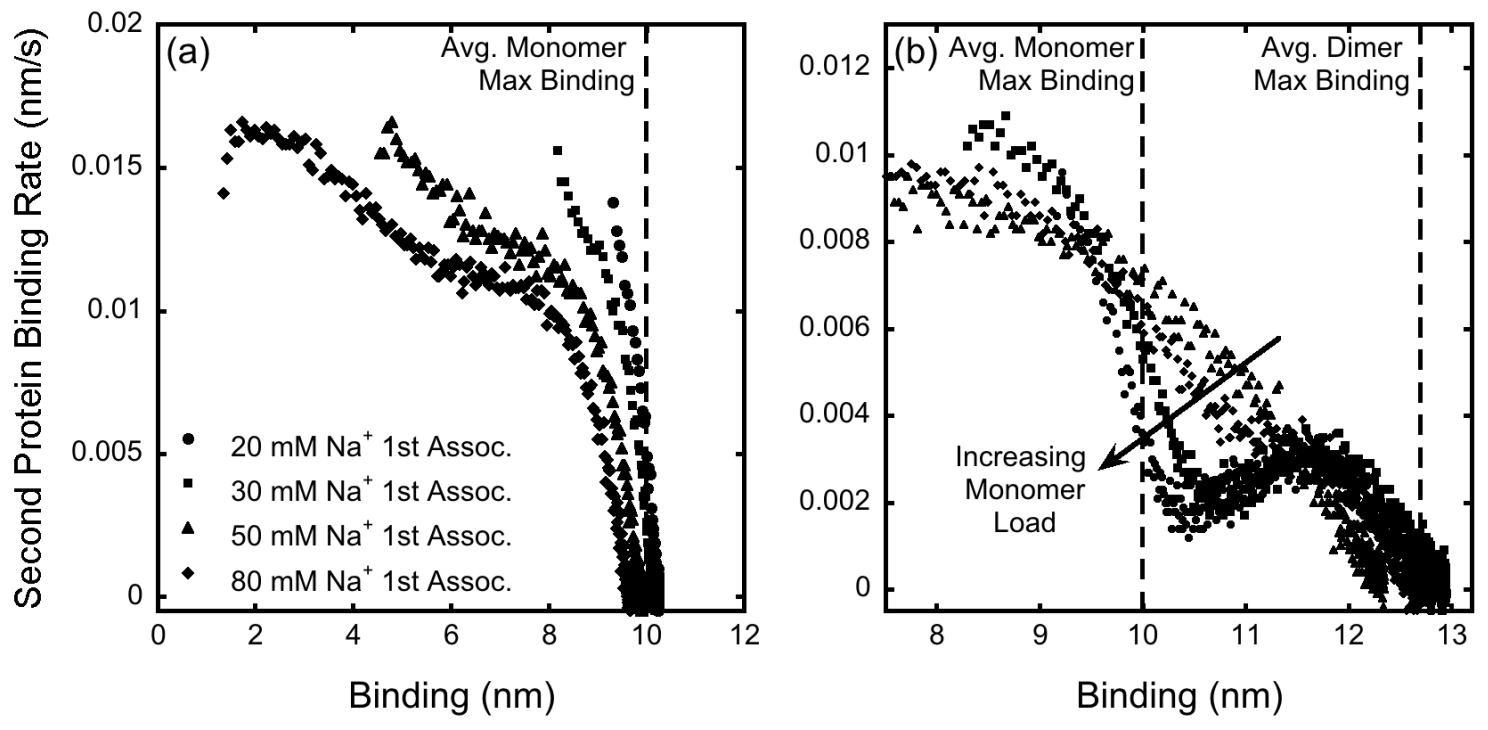

Figure 9.13. Binding rate during second association phase of sequential adsorption experiments depicted in Fig. 9.8 vs. total probe binding. Experimental conditions are the same as described in Fig. 9.8 
The regressed parameters from the association of monomer and dimer at varying salts suggest an association constant on the order of $10^{4}-10^{5} \mathrm{M}^{-1} \mathrm{~s}^{-1}$, values similar to that of antibody binding on Protein A domains (Jendeberg et al. 1995). Chapter 8 showed that for single component systems, the kinetics of surface adsorption is not a significant resistance during breakthrough experiments. Using the $k_{a}$ determined from the $20 \mathrm{mM} \mathrm{Na}^{+}$cases in this Chapter would result in a time-scale for binding, $\tau_{b}=1 /\left(k_{a} C\right)$, on the order of $1 \mathrm{~s}$ for $\sim 2 \mathrm{mg} / \mathrm{mL}$ solution of mAb monomer or dimer. This is significantly faster than the time-scales associated with diffusion limitations, $\tau_{D}=r_{p}^{2} / D_{e}$, which would be approximately $300 \mathrm{~s}$ for monomer or $600 \mathrm{~s}$ for dimer on the $50 \mu \mathrm{m}$ Nuvia HR-S resin. However, the kinetic resistances that arise from surface displacement in a two-component system may become significant. In the case of a monomer and dimer diffusing into a chromatographic resin, the lower diffusivity of the dimer will almost always result in the dimer encountering a surface with some degree of monomer loading. The rates of binding in Fig. 9.12b seem to imply that the rate of binding is an order of magnitude slower in the case where significant monomer is already bound to the surface. Significantly, this lowered rate is observed under conditions where the monomer concentration is essentially negligible. The presence of significant concentrations of monomer, as well as the pore architecture within a resin particle, suggest the actual rate of displacement observed in chromatographic experiments may be even slower. 

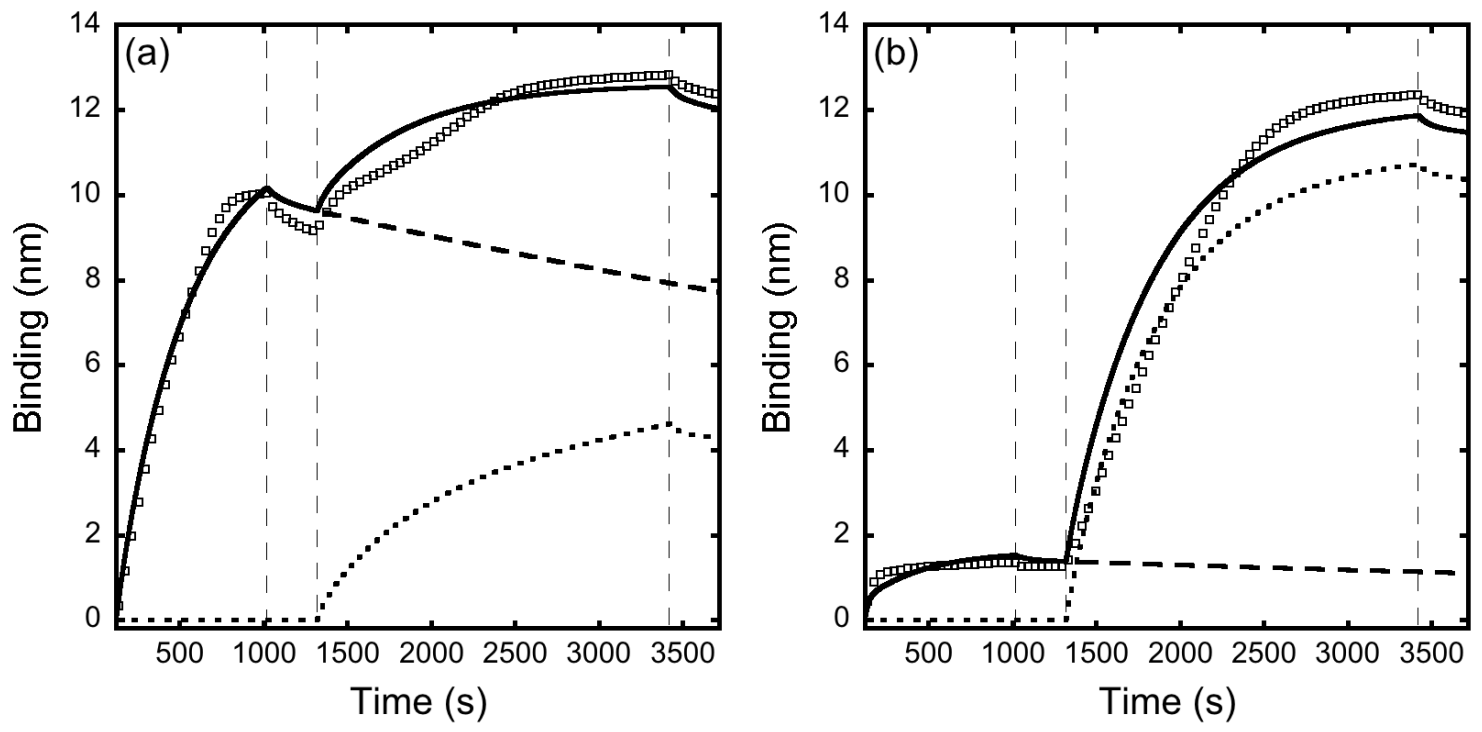

Figure 9.14. Experimental data (open squares) and model fits for a two-site binding model for sequential adsorption of $34 \mathrm{nM}$ antibody monomer in (a) $20 \mathrm{mM} \mathrm{Na}^{+}$and (b) $80 \mathrm{mM} \mathrm{Na}^{+}$ followed by $17 \mathrm{nM}$ antibody dimer in $20 \mathrm{mM} \mathrm{Na}^{+}$. Solid line is combined monomer and dimer model signal; dashed line is monomer model signal; dotted line is dimer model signal. Model lines based on numerical integration of Eq. 9.4 with parameters given in Table 9.1 


\section{Conclusions and Recommendations}

The results presented in this dissertation provide a description of the two-component adsorption behavior of a monoclonal antibody and its soluble dimer on a cation exchange resin. This included a physical characterization of the proteins and resin used, an examination of the single and two-component adsorption behavior at the particle level, the detailing of a practical separation scheme using frontal analysis based on the observed selectivities at the particle level, and a fundamental interrogation of competitive binding on an anionic surface using biolayer interferometry. More specific conclusions, as well as recommendations for further work pertaining to each of these topics are presented below.

\subsection{Single and Two-Component Adsorption Behavior}

These conclusions detail the work described in Chapters 6 and 7 of this dissertation. The results of these chapters show that the selectivity between a mAb monomer and dimer on a macroporous cation exchange resin varies substantially with salt concentration. For very dilute conditions, in the linear limit of the adsorption isotherm, LGE experiments yield selectivities that decrease with salt concentration from 8.7 to 2.2 in the range of salt concentrations where monomer and dimer are eluted. This trend is consistent with the behavior predicted by the stoichiometric displacement or mass action law model of ion exchange, which predicts that the affinity of the higher-charge ion (the dimer) for the resin is more strongly dependent on salt concentration that that of the lower-charge species (the monomer). For these conditions, since the salt concentration at elution is, for each component, a function of the steepness of the gradient, the salt gradient slope can be adjusted to optimize the separation.

Dramatically different results are obtained at lower salt concentrations and at high protein loads, as determined from batch adsorption equilibrium measurements. In this case, very similar equilibrium binding capacities are obtained on a mass basis for single component adsorption of monomer and dimer, despite the obviously large difference in molecular mass. This result suggests that for these strong binding conditions, the thickness of the adsorbed layer 
is essentially the same for each species, indicating a bound conformation where the dimer lies flat on the surface. The selectivity for two-component adsorption is also very low for these conditions, suggesting that interactions between either monomer or dimer species with the adsorbent surface are similar so that comparable amounts of monomer and dimer bind at equilibrium. Increasing the salt concentration weakens the binding, as shown by the decreasing steepness of the adsorption isotherm for each single component, but also improves the selectivity in two-component adsorption, increasingly favoring the dimer over the monomer. The reversal of the trend with respect to salt concentration relative to that observed under dilute conditions and higher salt concentrations in LGE experiments may be explained by crowding of the adsorbent surface. When binding is very strong, capacities close to complete monolayer coverage are attained and protein-protein interactions are the main determinants of selectivity. On the other hand, at high salt concentrations, the adsorbent surface is nearly protein free and protein-surface interactions control the selectivity according to the mass action law of ion exchange. At intermediate salt concentrations, both protein-protein and protein-surface interactions become important. Unfortunately, none of the currently available multicomponent adsorption equilibrium models seem to be capable of predicting these behaviors quantitatively based on single component isotherm data.

The adsorption kinetics is also affected by salt concentration. For the single component case, the kinetics is controlled by pore diffusion and similar effective diffusivities are obtained for non-binding and for strong binding conditions for either monomer or dimer. Whether under non-binding or strong binding conditions, the effective pore diffusivity is about twice as large for the monomer compared to the dimer. This result is due in part to the lower free solution diffusivity of the dimer and in part to its somewhere greater diffusional hindrance, both because of the larger size of the dimer. For conditions where binding is strong and the selectivity is low, simultaneous adsorption of monomer/dimer mixtures results in a single adsorption front within the particle. The apparent coupling of diffusion fluxes that seems to be responsible for the existence of a single front despite the dramatically different effective diffusivities is likely linked to the slow kinetics that can be expected for the exchange of bound 
monomer for dimer under conditions of nearly complete monolayer coverage that exist in this case. As the salt concentration is increased, however, and the system moves away from complete monolayer coverage, displacement of the bound monomer by the dimer occurs more readily and is no longer kinetically limited. In this case, simultaneous adsorption of monomer/dimer mixtures becomes diffusion-controlled and is described approximately by a dual-shrinking core mechanism. While the discrepancies between model and experimental results are significant, especially for the component present at a lower concentration, the model captures the experimental trends well and allows a prediction of the effects of varying operations conditions. As a corollary, the close agreement between the two-component kinetics data and the model, which assumes complete diffusion control, indicates that at the higher salt concentrations displacement of the monomer by the dimer occurs rapidly (i.e. on time scale much shorter than diffusion) even though binding is still relatively strong and molecular sizes are quite different.

These chapters examined the single and two-component behavior of monomer and dimer on a macroporous cation exchange resin. Many of the results are examined through the lens of diffusion within the macropores and competing for sites on the macropore surface. One recommendation would be to repeat some of these same experiments on polymerfunctionalized cation exchange resins. In polymer-functionalized resins, the internal pore structure is filled with charged polymers that greatly increase binding capacity and enhance diffusion rates into the resin. However, due to this functionalization, the mechanism of transport within these resins is quite different than in macroporous resins (Lenhoff 2011; PérezAlmodóvar et al. 2012; Tao et al. 2012). Repeating the experiments of these chapters on resins with a different transport mechanism may give further insight into what is driving the observed selectivity behavior in the macroporous resins. Whether all or only a subset of the same behaviors are observed (e.g. low selectivity at low salt concentration) in polymer-functionalized resins may also impact which resins are best suited to selectively remove the dimer using the frontal analysis techniques described in Chapter 8. 


\subsection{Frontal Analysis and Column Modeling}

These conclusions detail the work presented primarily in Chapter 8 of this dissertation. The ability to conduct mAb monomer-dimer separations by frontal analysis with a CEX resin was demonstrated for practical conditions. This required selecting buffer compositions where the binding strength is intermediate, thereby facilitating displacement of the monomer by the dimer. For these conditions, the binary breakthrough curves exhibit all of the characteristics expected for frontal analysis; namely, a pure monomer front reaching concentrations above the feed value, followed by a monomer-dimer front reaching the feed concentrations. Adsorption capacities determined from material balances show that the two front behaviors are qualitatively consistent with predictions from the SMA equilibrium model only at relatively high $\mathrm{Na}^{+}$concentrations. Poor agreement is found at low $\mathrm{Na}^{+}$, as for these conditions the SMA model predicts selectivity trends that are opposite of those observed experimentally. Fortunately, these are also conditions where the selectivity is low and thus practically unimportant.

The mechanistic model that was developed is limited to conditions where the SMA isotherm provides an adequate description of equilibrium, namely, at relatively high $\mathrm{Na}^{+}$concentrations. The model has predictive ability, but also provides insight about the factors that control the kinetics of the process. Comparison of model predictions and experimental results show that while intraparticle diffusion is the dominant contribution of band broadening, a kinetic resistance to binding must be considered. This resistance has only a small effect on single component breakthrough curves but deeply impacts the binary adsorption behavior. Thus, it must be included in the model in order to quantitatively predict binary breakthrough and hence the purity and yield attainable by frontal analysis. Comparison of model predictions with previously obtained batch uptake kinetics data generated by confocal microscopy confirm the validity of this conclusion. No attempt was made to model the binary breakthrough curves at low $\mathrm{Na}^{+}$concentrations. Although these conditions did not lead to a practical separation, modeling this behavior is interesting from a fundamental viewpoint. One possibility is that the predictions for these conditions failed because the SMA isotherm is simply unable to describe the relevant binary adsorption equilibria. Another possibility is that the binding kinetics model 
is overly simplistic. The work presented in Chapter 9 attempted to independently measure the competitive binding equilibrium and kinetics with the goal of developing more accurate kinetic models that could explain the unexpected selectivity trends as a function of counterion concentration.

From a more practical viewpoint, the results show that frontal analysis can be developed as a useful tool to remove aggregates from mAb feedstock. Compared to ordinary bind-elute modalities, frontal analysis has the advantage of more fully utilizing the resin binding capacity and minimizing dilution of the purified product. Resin with relatively small particle size and with relatively large pores are needed for such a process in order to minimize band-broadening effects. One recommendation in pushing this work further would be to explore alternative types of chromatographic media such as monoliths or membranes, where mass transfer is not rate limiting. Predicting performance, in these cases, would however require a description of other band-broadening factors which affect these systems (film mass transfer and axial dispersion), in addition to the kinetic resistances associated with the exchange of bound protein molecules observed in this work.

Another recommendation would be to attempt to implement the two-component frontal analysis technique in pursuit of a continuous chromatography process. One implementation of this could be a CaptureSMB-type system (Angarita et al. 2014), in which two columns are utilized. In such a separation scheme, the first column would be loaded with feedstock throughout the entirety of the first breakthrough front, with only pure monomer leaving the column. Just prior to breakthrough of the second front of monomer-dimer feed, the feed stream would be switched to the second column, which would be loaded in the same way. During loading on the second column, the first column would be eluted and prepped for the next round of loading. The modeling and sizing of columns to achieve an efficient separation that can be run indefinitely in this manner could be of practical importance for next generation processes. 


\subsection{Biolayer Interferometry}

These conclusions detail the work primarily described in Chapter 9 of this dissertation. This work demonstrated the use of biolayer interferometry to isolate and observe kinetic surface effects and relate them to results from chromatographic media. AR2G probes were shown to function as a weak cation exchanger, allowing for comparisons to cation exchange chromatography resins when operated at $\mathrm{pHs}$ where they would be fully deprotonated. The observed binding response of an antibody monomer and its soluble dimer were shown to be consistent with an ion-exchange resin surface; namely, a decreasing binding response as the binding is modulated by $\mathrm{Na}^{+}$, and a stronger response for the higher-charge dimer at all conditions observed.

Importantly, the BLI technique allowed for the observation of kinetic effects associated with the surface exchange of the antibody monomer and dimer. For surfaces with a large amount of monomer prebound, the uptake rate of a subsequent dimer step is seen to be significantly slower than for conditions where no or little monomer is initially present. That this reduced rate is observed with no monomer present suggests that the actual rate of exchange encountered under more practical conditions may be even slower. The results also support work of prior chapters, which showed that the effect of the kinetic resistance needed to describe the monomer and dimer behavior decreased as the salt concentration was increased. This may be explained by the reduced monomer loading on the surface at these higher salt concentrations.

From a practical perspective, the results suggest that these kinetic limitations are less important in single component systems. Additionally, many of these effects may only be observed under very high loading conditions, such as in the frontal mode described in Chapter 8. However, as impurity profiles become more severe, and the push is made for higher resin utilization, these effects may become important. Additionally, for systems where other mass transfer resistances are less significant (e.g. monoliths or membranes), these effects are likely to be more pronounced and should be taken into account when designing the step. 
A recommendation in furthering the work of this chapter could be working with Pall ForteBio to design or produce probes with strong ion exchange ligands. This would allow examining operating conditions that are not suitable for a weak cation surface (e.g. pH 5.0), but are of practical importance when designing chromatographic processes. An additional recommendation would be to attempt to further bridge the gap between the idealized surface of the BLI experiments and chromatographic media. Again, the use of alternative types of chromatographic media such as monoliths or ion-exchange membranes where diffusional resistances are not a significant factor may lend insight into how much the internal pore architecture of macroporous resins contribute to the kinetic resistances observed during macromolecule exchange.

Additionally, a more rigorous characterization of the actual biosensor probes would be of value. The binding along the length of the tip in Fig. 9.3b was unexpected, and may be of concern if it occurs for all biosensors. While this binding along the length may not impact the actual measurements (due to the design of the optics within the biosensor), it may impact the protein concentration in solution, particular at very low concentrations. Differentiating between the type of binding on the surface vs. along the length may require some clever experimental design, but would be worth further investigation.

Finally, BLI provides a relatively simple tool that is able to detect and quantify difficult to observe phenomena. However, the relatively simple models used in this work are unable to capture some of the complexities in the binding process, particularly during sequential adsorption experiments. Coupling BLI experiments with more complex biophysical modeling may lend insight into the origin of these difficult to quantitate surface interactions, particularly in multi-component systems, while also providing experimentally verifiable corroboration. 


\section{Works Referenced}

Angarita, M., Müller-Späth, T., Baur, D., Lievrouw, R., Lissens, G. \& Morbidelli, M. Twincolumn CaptureSMB: a novel cyclic process for Protein A affinity chromatography. J. Chromatogr. A 1389, 85-95 (2015).

Ansaldi, D. A. \& Lester, P. Separation of Polypeptide Monomers. U.S. Patent 6,620,918 B2 (2003).

Borg, N., Brodsky, Y., Moscariello, J., Vunnum, S., Vedantham, G., Westerberg, K. \& Nilsson, B. Modeling and robust pooling design of a preparative cation-exchange chromatography step for purification of monoclonal antibody monomer from aggregates. J. Chromatogr. A 1359, 170-181 (2014).

Bregenholt, S., Jensen, A., Lantto, J., Hyldig, S. \& Haurum, J. S. Recombinant human polyclonal antibodies: A new class of therapeutic antibodies against viral infections. Curr. Pharm. Des. 12, 2007-15 (2006).

Brooks, C. A. \& Cramer, S. M. Steric mass-action ion exchange: Displacement profiles and induced salt gradients. AIChE J. 38, 1969-1978 (1992).

Carta, G., Ubiera, A. R. \& Pabst, T. M. Protein mass transfer kinetics in ion exchange media: measurements and interpretations. Chem. Eng. Technol. 28, 1252-1264 (2005).

Carta, G. \& Jungbauer, A. Protein Chromatography: Process Development and Scale-Up. (Wiley-VCH GmbH \& Co., 2010).

Carter, P. J. Introduction to current and future protein therapeutics: a protein engineering perspective. Exp. Cell Res. 317, 1261-9 (2011).

Casadevall, A. \& Scharff, M. D. Return to the past: the case for antibody-based therapies in infectious diseases. Clin. Infect. Dis. 21, 150-61 (1995).

Chen, D.-J. Fiber optic direct-sensing bioprobe using a phase-tracking approach. U.S. Patent 5,804,453 (1998).

Chen, S., Lau, H., Brodsky, Y., Kleemann, G. R. \& Latypov, R. F. The use of native cationexchange chromatography to study aggregation and phase separation of monoclonal antibodies. Protein Sci. 19, 1191-204 (2010).

Chmielowski, R., Meissner, S., Roush, D., Linden, T. O., Glowacki, E., Konietzko, J. \& NtiGyabaah, J. Resolution of heterogeneous charged antibody aggregates via 
multimodal chromatography: A comparison to conventional approaches. Biotechnol. Prog. 30, 636-645 (2014).

Concepcion, J., Witte, K., Wartchow, C., Choo, S., Yao, D., Persson, H., Wei, J., Li, P., Heidecker, B., Ma, W., Varma, R., Zhao, L.-S., Perillat, D., Carricato, G., Recknor, M., Du, K., Ho, H., Ellis, T., Gamez, J., Howes, M., Phi-Wilson, J., Lockard, S., Zuk, R. \& Tan, H. Label-free detection of biomolecular interactions using biolayer interferometry for kinetic characterization. Comb. Chem. High Throughput Screen. 12, 791-800 (2009).

Cromwell, M. E. M., Hilario, E. \& Jacobson, F. Protein aggregation and bioprocessing. AAPS J. 8, E572-9 (2006).

Dengl, S., Wehmer, M., Hesse, F., Lipsmeier, F., Popp, O. \& Lang, K. Aggregation and chemical modification of monoclonal antibodies under upstream processing conditions. Pharm. Res. 30, 1380-1399 (2013).

DePhillips, P. \& Lenhoff, A. M. Determinants of protein retention characteristics on cation-exchange adsorbents. J. Chromatogr. A 933, 57-72 (2001).

Do. T., Ho, F., Heidecker, B., Witte, K., Chang, L. \& Lerner, L. A rapid method for determining dynamic binding capacity of resins for the purification of proteins. Protein Expr. Purif. 60, 147-150 (2008).

Ecker, D. M., Jones, S. D. \& Levine, H. L. The therapeutic monoclonal antibody market. $m A b s$ 7, 9-14 (2015).

Edwards, P. R., Maule, C. H., Leatherbarrow, R. J. \& Winzor, D. J. Second-order kinetic analysis of IAsys biosensor data: its use and applicability. Anal. Biochem. 263, 112 (1998).

Evaluate Ltd. EvaluatePharma World Preview 2016, Outlook to 2022. 1-39 (2016). Available at: http://info.evaluategroup.com/rs/607-YGS-364/images/wp16.pdf.

Fahrner, R. L., Knudsen, H. L., Basey, C. D., Galan, W., Feuerhelm, D., Vanderlaan, M. \& Blank, G. S. Industrial Purification of Pharmaceutical Antibodies: Development, Operation, and Validation of Chromatography Processes. Biotechnol. Genet. Eng. Rev. 18, 301-327 (2001).

Fargues, C., Bailly, M. \& Grevillot, G. Adsorption of BSA and hemoglobin on hydroxyapatite support: Equilibria and multicomponent dynamic adsorption. Adsorption 4, 5-16 (1998). 
Fischer, M. J. E., Amine coupling through EDC/NHS: A practical approach, in: Surf. Plasmon Reson. Methods Protoc. 55-73 (2010).

Food and Drug Administration. Guidance for industry Q5A viral safety evaluation derived from cell lines of human or animal origin guidance for industry. 1-33 (1998).

Food and Drug Administration. Guidance for industry : Q6B specifications : test procedures and acceptance criteria for biotechnological/biological products. 120 (1999).

Forrer, N., Butté, A. \& Morbidelli, M. Chromatographic behavior of a polyclonal antibody mixture on a strong cation exchanger column. Part I: Adsorption characterization. J. Chromatogr. A 1214, 59-70 (2008).

Forrer, N., Butté, A. \& Morbidelli, M. Chromatographic behavior of a polyclonal antibody mixture on a strong cation exchanger column. Part II: Adsorption modelling. J. Chromatogr. A 1214, 71-80 (2008).

Frisken, B. J. Revisiting the method of cumulants for the analysis of dynamic lightscattering data. Appl. Opt. 40, 4087-4091 (2001).

Gadam, S. D., Gallant, S. R. \& Cramer, S. M. Transient profiles in ion-exchange displacement chromatography. AlChE J. 41, 1676-1686 (1995).

Gagnon, P. Technology trends in antibody purification. J. Chromatogr. A 1221, 57-70 (2012).

Gallant, S. R., Vunnum, S. \& Cramer, S. M. Modeling gradient elution of proteins in ionexchange chromatography. AIChE J. 42, 2511-2520 (1996).

Garke, G., Hartmann, R., Papamichael, N., Deckwer, W.-D. \& Anspach, F. B. The Influence of Protein Size on Adsorption Kinetics and Equilibria in Ion-Exchange Chromatography. Sep. Sci. Technol. 34, 2521-2538 (1999).

Goeddel, D. V., Kleid, D. G., Bolivar, F., Heyneker, H. L., Yansura, D. G., Crea, R., Hirose, T., Kraszewski, A., Itakura, K. \& Riggs, A. D. Expression in Escherichia coli of chemically synthesized genes for human insulin. Proc. Natl. Acad. Sci. U. S. A. 76, 106-10 (1979).

Grand View Research Inc. Monoclonal antibodies (mAbs) market analysis, 2013-2024. (2016). Available at: http://www.grandviewresearch.com/industryanalysis/monoclonal-antibodies-market. 
Gu, T., Tsai, G.-J. \& Tsao, G. T. Multicomponent adsorption and chromatography with uneven saturation capacities. AIChE J. 37, 1333-1340 (1991).

Guo, J., Zhang, S. \& Carta, G. Unfolding and aggregation of a glycosylated monoclonal antibody on a cation exchange column. Part I. Chromatographic elution and batch adsorption behavior. J. Chromatogr. A 1356, 117-28 (2014).

Guo, J. \& Carta, G. Unfolding and aggregation of monoclonal antibodies on cation exchange columns: Effects of resin type, load buffer, and protein stability. J. Chromatogr. A 1388, 184-194 (2015).

Hagel, L., Östberg, M. \& Andersson, T. Apparent pore size distributions of chromatography media. J. Chromatogr. A 743, 33-42 (1996).

Hanke, A. T. \& Ottens, M. Purifying biopharmaceuticals: Knowledge-based chromatographic process development. Trends Biotechnol. 32, 210-220 (2014).

Haurum, J. S. Recombinant polyclonal antibodies: the next generation of antibody therapeutics? Drug Discov. Today 11, 655-60 (2006).

Hayhurst, A. \& Georgiou, G. High-throughput antibody isolation. Curr. Opin. Chem. Biol. 5, 683-9 (2001).

Hubbuch, J., Linden, T., Knieps, E., Thömmes, J. \& Kula, M.-R. Dynamics of protein uptake within the adsorbent particle during packed bed chromatography. Biotechnol. Bioeng. 80, 359-68 (2002).

Hubbuch, J., Linden, T., Knieps, E., Ljunglöf, A., Thömmes, J. \& Kula, M.-R. Mechanism and kinetics of protein transport in chromatographic media studied by confocal laser scanning microscopy Part I. The interplay of sorbent structure and fluid phase conditions. J. Chromatogr. A 1021, 93-104 (2003).

Hubbuch, J., Linden, T., Knieps, E., Thömmes, J. \& Kula, M.-R. Mechanism and kinetics of protein transport in chromatographic media studied by confocal laser scanning microscopy Part II. Impact on chromatographic separations. J. Chromatogr. A 1021, 105-115 (2003).

Hunter, A. K. \& Carta, G. Protein adsorption on novel acrylamido-based polymeric ionexchangers. I. Morphology and equilibrium adsorption. J. Chromatogr. A 897, 65-80 (2000).

Imai, K. \& Takaoka, A. Comparing antibody and small-molecule therapies for cancer. Nat. Rev. Cancer 6, 714-27 (2006). 
Jarvis, L. M. The Year In New Drugs 2014. Chem. Eng. News 93, 11-16 (2015).

Jarvis, L. M. The Year In New Drugs 2015. Chem. Eng. News 94, 12-17 (2016).

Jarvis, L. M. The Year In New Drugs 2016. Chem. Eng. News 95, 28-32 (2017).

Jendeberg, L., Persson, B., Andersson, R., Karlsson, R., Uhlén, M. \& Nilsson, B. Kinetic analysis of the interaction between Protein A domain variants and human FC using plasmon resonance detection. J. Mol. Recognit. 8, 270-278 (1995).

Johnson, R. D. \& Arnold, F. H. The temkin isotherm describes heterogeneous protein adsorption. Biochim. Biophys. Acta (BBA)/Protein Struct. Mol. 1247, 293-297 (1995).

Johnson, R. D. \& Arnold, F. H. Review: multipoint binding and heterogeneity in immobilized metal affinity chromatography. Biotechnol. Bioeng. 48, 437-443 (1995).

Jungbauer, A., Machold, C. \& Hahn, R. Hydrophobic interaction chromatography of proteins III. Unfolding of proteins upon adsorption. J. Chromatogr. A 1079, 221228 (2005).

Karlsson, R., Michaelsson, A. \& Mattsson, L. Kinetic analysis of monoclonal antibodyantigen interactions with a new biosensor based analytical system. J. Immunol. Methods. 145, 229-240 (1991).

Kelley, B. D., Switzer, M., Bastek, P., Kramarczyk, J. F., Molnar, K., Yu, T. \& Coffman, J. High-throughput screening of chromatographic separations: IV. Ion-exchange. Biotechnol. Bioeng. 100, 950-63 (2008).

Kelley, B. D. Industrialization of mAb production technology: the bioprocessing industry at a crossroads. MAbs 1, 443-52 (2009).

Kopaciewicz, W., Rounds, M. A., Fausnaugh, J. \& Regnier, F. E. Retention model for highperformance ion-exchange chromatography. J. Chromatogr. 266, 3-21 (1983).

Koppel, D. E. Analysis of Macromolecular Polydispersity. J. Chem. Phys. 57, 4814-4820 (1972).

Kramarczyk, J. F., Kelley, B. D. \& Coffman, J. L. High-throughput screening of chromatographic separations: II. Hydrophobic interaction. Biotechnol. Bioeng. 100, 707-20 (2008). 
Kunert, R. \& Reinhart, D. Advances in recombinant antibody manufacturing. Appl. Microbiol. Biotechnol. 100, 3451-3461 (2016).

Leavy, O. Therapeutic antibodies: past, present and future. Nat. Rev. Immunol. 10, 297297 (2010).

Leader, B., Baca, Q. J. \& Golan, D. E. Protein therapeutics: a summary and pharmacological classification. Nat. Rev. Drug Discov. 7, 21-39 (2008).

Lenhoff, A. M. Protein adsorption and transport in polymer-functionalized ionexchangers. J. Chromatogr. A 1218, 8748-59 (2011).

Levy, N. E., Valente, K. N., Choe, L. H., Lee, K. H. \& Lenhoff, A. M. Identification and characterization of host cell protein product-associated impurities in monoclonal antibody bioprocessing. Biotechnol. Bioeng. 111, 904-912 (2014).

Lewus, R. K. \& Carta, G. Binary protein adsorption on gel-composite ion-exchange media. AlChE J. 45, 512-522 (1999).

Liao, H.-X., Levesque, M. C., Nagel, A., Dixon, A., Zhang, R., Walter, E., Parks, R., Whitesides, J., Marshall, D. J., Hwang, K.-K., Yang, Y., Chen, X., Gao, F., Munshaw, S., Kepler, T. B., Denny, T., Moody, M. A. \& Haynes, B. F. High-throughput isolation of immunoglobulin genes from single human $B$ cells and expression as monoclonal antibodies. J. Virol. Methods 158, 171-9 (2009).

Linden, T., Ljunglöf, A., Kula, M.-R. \& Thömmes, J. Visualizing two-component protein diffusion in porous adsorbents by confocal scanning laser microscopy. Biotechnol. Bioeng. 65, 622-630 (1999).

Liu, H. F., McCooey, B., Duarte, T., Myers, D. E., Hudson, T., Amanullah, A., Van Reis, R. \& Kelley, B. D. Exploration of overloaded cation exchange chromatography for monoclonal antibody purification. J. Chromatogr. A 1218, 6943-6352 (2011).

Liu, J. K. H. The history of monoclonal antibody development - Progress, remaining challenges and future innovations. Ann. Med. Surg. 3, 113-116 (2014).

Ljunglöf, A. \& Hjorth, R. Confocal microscopy as a tool for studying protein adsorption to chromatographic matrices. J. Chromatogr. A 743, 75-83 (1996).

Ljunglöf, A. \& Thömmes, J. Visualising intraparticle protein transport in porous adsorbents by confocal microscopy. J. Chromatogr. A 813, 387-395 (1998).

Lofas, S., Malmqvist, M., Rönnberg, I., Stenberg, E., Liedberg, B. \& Lundström, I. Bioanalysis with surface plasmon resonance. Sensors Actuators B 5, 79-84 (1991). 
Lonberg, N. Human antibodies from transgenic animals. Nat. Biotechnol. 23, 1117-25 (2005).

Lonberg, N. Fully human antibodies from transgenic mouse and phage display platforms. Curr. Opin. Immunol. 20, 450-9 (2008).

Low, D., O’Leary, R. \& Pujar, N. S. Future of antibody purification. J. Chromatogr. B. Analyt. Technol. Biomed. Life Sci. 848, 48-63 (2007).

Martin, C., Iberer, G., Ubiera, A. R. \& Carta, G. Two-component protein adsorption kinetics in porous ion exchange media. J. Chromatogr. A 1079, 105-115 (2005).

Mattila, J. Clark, M., Liu, S., Pieracci, J., Gervais, T. R., Wilson, E., Galperina, O., Li, Xinfang., Roush, D., Zoeller, K., Brough, H. \& Simpson-Platre, C. Retrospective Evaluation of Low-pH Viral Inactivation and Viral Filtration Data from a Multiple Company Collaboration. PDA J. Pharm. Sci. Technol. 70, 293-299 (2016).

Naik, S., Brock, S., Akkaladevi, N., Tally, J., Mcginn-Straub, W., Zhang, N., Gao, P., Gogol, E. P., Pentelute, B. L., Collier, R. J. \& Fisher, M. T. Monitoring the kinetics of the $\mathrm{pH}$ driven transition of the anthrax toxin prepore to the pore by biolayer interferometry and surface plasmon resonance. Biochemistry 52, 6335-6347 (2013).

Nielsen, L. S., Baer, A., Müller, C., Gregersen, K., Mønster, N. T., Rasmussen, S. K., Weilguny, D. \& Tolstrup, A. B. Single-batch production of recombinant human polyclonal antibodies. Mol. Biotechnol. 45, 257-66 (2010).

Norman, P. Monoclonal antibodies in the pipeline: A segment of major growth. Report executive summary. Insight Pharma Reports 2013, 5-9 (2011).

O’Shannessy, D. J., Brigham-Burke, M., Soneson, K. K., Hensley, P. \& Brooks, I. Determination of rate and equilibrium binding constants for macromolecular interactions using surface plasmon resonance: Use of nonlinear least squares analysis methods. Anal. Biochem. 212, 457-468 (1993).

O'Shannessy, D. J. Determination of kinetic rate and equilibrium binding constants for macromolecular interactions: a critique of the surface plasmon resonance literature. Curr. Opin. Biotechnol. 5, 65-71 (1994).

Önell, A. \& Andersson, K. Kinetic determinations of molecular interactions using Biacore - minimum data requirements for efficient experimental design. J. Mol. Recognit. 18, 307-317 (2005). 
Pabst, T. M., Suda, E. J., Thomas, K. E., Mensah, P., Ramasubramanyan, N., Gustafson, M. E. \& Hunter, A. K. Binding and elution behavior of proteins on strong cation exchangers. J. Chromatogr. A 1216, 7950-6 (2009).

Pall ForteBio, Technical note 26: Dip and read amine reactive second-generation (AR2G) biosensors. (2011). Available at: http://www.fortebio.com/biosensorsAR2G.html.

Pall ForteBio, Technical note 27: Dip and read amine reactive second-generation (AR2G) reagent kit. (2011). Available at http://www.fortebio.com/biosensorsAR2G.html.

Pérez-Almodóvar, E. X., Tao, Y. \& Carta, G. Protein adsorption and transport in cation exchangers with a rigid backbone matrix with and without polymeric surface extenders. Biotechnol. Prog. 27, 1264-72 (2011).

Pérez-Almodóvar, E. X., Wu, Y. \& Carta, G. Multicomponent adsorption of monoclonal antibodies on macroporous and polymer grafted cation exchangers. J. Chromatogr. A 1264, 48-56 (2012).

Pharmaceutical Research and Manufacturers of America. 2012 Pharmaceutical industry profile. (2012). Available at:

http://www.phrma.org/sites/default/files/pdf/phrma_industry_profile.pdf.

Reichert, J. M. Trends in development and approval times for new therapeutics in the United States. Nat. Rev. Drug Discov. 2, 695-702 (2003).

Reichert, J. M., Rosensweig, C. J., Faden, L. B. \& Dewitz, M. C. Monoclonal antibody successes in the clinic. Nat. Biotechnol. 23, 1073-8 (2005).

Reichert, J. M. Which are the antibodies to watch in 2013? MAbs 5, 1-4 (2013).

Reichert, J. M. Therapeutic monoclonal antibodies approved or in review in the European Union or United States. The Antibody Society (2013).

Reichert, J. M. The Antibody Society: Archives For Approvals. (2017). Available at: http://www.antibodysociety.org/category/approvals/.

Rosenberg, A. S. Effects of protein aggregates: an immunologic perspective. AAPS J. 8, E501-7 (2006).

Roth, C. M., Unger, K. K. \& Lenhoff, A. M. Mechanistic model of retention in protein ionexchange chromatography. J. Chromatogr. A 726, 45-56 (1996). 
Ruthven, D. M. Principles of Adsorption and Adsorption Processes. (John Wiley \& Sons, Inc., 1983).

Schneider, C. A., Rasband, W. S. \& Eliceiri, K. W. NIH Image to ImageJ: 25 years of image analysis. Nat. Methods 9, 671-675 (2012).

Schuck, P. Use of surface plasmon resonance to probe the equilibrium and dynamic aspects of interactions between biological macromolecules. Annu. Rev. Biophys. Biomol. Struct. 26, 541-566 (1997).

Shukla, A. A, Hinckley, P. J., Gupta, P., Yigzaw, Y. \& Hubbard, B. Strategies To Address Aggregation During Protein A Chromatography. Bioprocess Int. 3, 36-44 (2005).

Shukla, A. A., Hubbard, B., Tressel, T., Guhan, S. \& Low, D. Downstream processing of monoclonal antibodies--application of platform approaches. J. Chromatogr. $B$ 848, 28-39 (2007).

Shukla, A. A. \& Hinckley, P. J. Host cell protein clearance during Protein A chromatography: Development of an improved column wash Step. Biotechnol. Prog. 24, 1115-1121 (2008).

Staby, A., Jacobsen, J. H., Hansen, R. G., Bruus, U. K. \& Jensen, I. H. Comparison of chromatographic ion-exchange resins $\mathrm{V}$. Strong and weak cation-exchange resins. J. Chromatogr. A 1118, 168-79 (2006).

Striegel, A. M., Yau, W. W., Kirkland, J. J. \& Bly, D. D. in Modern Size-Exclusion Liquid Chromatography: Practice of Gel Permeation and Gel Filtration Chromatography 18-48 (2009).

Suda, E. J., Thomas, K. E., Pabst, T. M., Mensah, P., Ramasubramanyan, N., Gustafson, M. E. \& Hunter, A. K. Comparison of agarose and dextran-grafted agarose strong ion exchangers for the separation of protein aggregates. J. Chromatogr. A 1216, 5256-5264 (2009).

Tan, H., Tan, Y., Witte, K. L., Zuk, R., Carricato, G. L. \& Lockard, S. Fiber-optic assay apparatus based on phase-shift interferometry. U.S. Patent 7,319,525 B2 (2008).

Tao, Y., Carta, G., Ferreira, G. \& Robbins, D. Adsorption of deamidated antibody variants on macroporous and dextran-grafted cation exchangers: I. Adsorption equilibrium. J. Chromatogr. A 1218, 1519-29 (2011).

Tao, Y., Carta, G., Ferreira, G. \& Robbins, D. Adsorption of deamidated antibody variants on macroporous and dextran-grafted cation exchangers: II. Adsorption kinetics. J. Chromatogr. A 1218, 1530-1537 (2011). 
Tao, Y., Pérez-Almodóvar, E. X., Carta, G., Ferreira, G. \& Robbins, D. Adsorption kinetics of deamidated antibody variants on macroporous and dextran-grafted cation exchangers. III. Microscopic studies. J. Chromatogr. A 1218, 8027-35 (2011).

Tao, Y., Chen, N., Carta, G., Ferreira, G. \& Robbins, D. Modeling multicomponent adsorption of monoclonal antibody charge variants in cation exchange columns. AlChE J. 58, 2503-2511 (2012).

Teske, C. A., Schroeder, M., Simon, R. \& Hubbuch, J. Protein-labeling effects in confocal laser scanning microscopy. J. Phys. Chem. B 109, 13811-7 (2005).

Teske, C. A., Von Lieres, E., Schröder, M., Ladiwala, A., Cramer, S. M. \& Hubbuch, J. J. Competitive Adsorption of Labeled and Native Protein in Confocal Laser Scanning Microscopy. Biotechnol. Bioeng. 95, 58-66 (2006).

Teske, C. A., Simon, R., Niebisch, A. \& Hubbuch, J. Changes in retention behavior of fluorescently labeled proteins during ion-exchange chromatography caused by different protein surface labeling positions. Biotechnol. Bioeng. 98, 193-200 (2007).

Toueille, M., Uzel, A., Depoisier, J. F. \& Gantier, R. Designing new monoclonal antibody purification processes using mixed-mode chromatography sorbents. J. Chromatogr. B 879, 836-843 (2011).

Vázquez-Rey, M. \& Lang, D. A. Aggregates in monoclonal antibody manufacturing processes. Biotechnol. Bioeng. 108, 1494-1508 (2011).

Velayudhan, A. \& Horvath, C. Preparative Chromatography of Proteins Analysis of the Multivalent Ion-Exchange Formalism. 443, 13-29 (1988).

Verzijl, D., Riedl, T., Parren, P. W. H. I. \& Gerritsen, A. F. A novel label-free cell-based assay technology using biolayer interferometry. Biosens. Bioelectron. 87, 388395 (2017).

Vicente, T., Mota, J. P. B., Peixoto, C., Alves, P. M. \& Carrondo, M. J. T. Modeling protein binding and elution over a chromatographic surface probed by surface plasmon resonance. J. Chromator. A 1217, 2032-2041 (2010).

Vicente, T., Mota, J. P. B., Peixoto, C., Alves, P. M. \& Carrondo, M. J. T. Analysis of adsorption of a baculovirus bioreaction bulk on an ion-exchange surface by surface plasmon resonance. J. Biotechnol.148, 171-181 (2010). 
Wang, W., Singh, S. K., Zeng, D. L., King, K. \& Nema, S. Antibody structure, instability, and formulation. J. Pharm. Sci. 96, 1-26 (2007).

Weinbrenner, W. F. \& Etzel, M. R. Competitive adsorption of $\alpha$-lactalbumin and bovine serum albumin to a sulfopropyl ion-exchange membrane. J. Chromatogr. A 662, 414-419 (1994).

Wiberg, F. C., Rasmussen, S. K., Frandsen, T. P., Rasmussen, L. K., Tengbjerg, K., Coljee, V. W., Sharon, J., Yang, C.-Y., Bregenholt, S., Nielsen, L. S., Haurum, J. S. \& Tolstrup, A. B. Production of target-specific recombinant human polyclonal antibodies in mammalian cells. Biotechnol. Bioeng. 94, 396-405 (2006).

Wilson, E. J. \& Geankoplis, C. J. Liquid Mass Transfer at Very Low Reynolds Numbers in Packed Beds. Ind. Eng. Chem. Fundam. 5, 9-14 (1966).

Wurm, F. M. Production of recombinant protein therapeutics in cultivated mammalian cells. Nat. Biotechnol. 22, 1393-8 (2004).

Xu, X. \& Lenhoff, A. M. Binary adsorption of globular proteins on ion-exchange media. J. Chromatogr. A 2009, 6177-6195 (2009).

Yamamoto, S., Nakanishi, K., Matsuno, R. \& Kamikubo, T. Ion exchange chromatography of proteins-prediction of elution curves and operating conditions. I. Theoretical considerations. Biotechnol. Bioeng. 25, 1465-83 (1983).

Yu, Y., Mitchell, S., Lynaugh, H., Brown, M., Nobrega, R. P., Zhi, X., Sun, T., Caffry, I., Cao, Y., Yang, R., Burnina, I., Xu, Y. \& Estep, P. Understanding ForteBio's sensors for high-throughput kinetic and epitope screening for purified antibodies and yeast culture supernatant. J. Biomol. Screen. 21, 88-95 (2015).

Zeder-Lutz, G., Altschuh, D., Geysen, H. M., Trifilieff, E., Sommermeyer, G. \& Van Regenmortel, M. H. V. Monoclonal antipeptide antibodies: affinity and kinetic rate constants measured for the peptide and the cognate protein using a biosensor technology. Mol. Immunol. 30, 145-155 (1993).

Zhang, J., Kleinöder, T. \& Gasteiger, J. Prediction of pKa values for alpiphatic carboxylic acids and alcohols with empirical atomic charge descriptors. J. Chem. Inf. Model. 46, 2256-2266 (2006).

Zhou, J. X., Dermawan, S., Solamo, F., Flynn, G., Stenson, R., Tressel, T. \& Guhan, S. pHconductivity hybrid gradient cation-exchange chromatography for process-scale monoclonal antibody purification. J. Chromatogr. A 1175, 69-80 (2007). 
Zhu, M. \& Carta, G. Adsorption of polyethylene-glycolated bovine serum albumin on macroporous and polymer-grafted anion exchangers. J. Chromatogr. A 1326, 2938 (2014). 


\section{Appendix A: Derivation of Finite-Difference Discretization Scheme}

The frontal analysis modeling in MATLAB utilizes a finite-difference discretization along the column length, within the particle radius, and relates the fluid phase concentration to the adsorbed phase concentration with an isotherm model. The 1-D column conservation equation ignoring axial dispersion is given by equation A.1 with the following boundary and initial conditions:

$$
\begin{aligned}
& \varepsilon \frac{\partial C_{i}}{\partial t}+(1-\varepsilon) \frac{\partial\left\langle\bar{q}_{i}\right\rangle}{\partial t}+u \frac{\partial C_{i}}{\partial x}=0 \\
& C_{i}=C_{i, F} \text { at } x=0 \text { for } t>0 \\
& C_{i}=\left\langle\bar{q}_{i}\right\rangle=0 \text { at } t=0
\end{aligned}
$$

where $\varepsilon$ is the column porosity, $C$ is the fluid phase concentration, $\left\langle\bar{q}_{i}\right\rangle$ is the average concentration within the stationary phase (including concentration within the pore volume), and $u$ is the linear velocity. The rate equation for pore diffusion in a spherical particle is given by equation A.2 with the following initial and boundary conditions:

$$
\begin{aligned}
& \frac{\partial q_{i}}{\partial t}+\varepsilon_{p} \frac{\partial c_{i}}{\partial t}=\frac{1}{r^{2}} \frac{\partial}{\partial r}\left(D_{e, i} r^{2} \frac{\partial c_{i}}{\partial r}\right) \\
& c_{i}=q_{i}=0 \text { at } t=0 \\
& \frac{4}{3} \pi r^{3} \frac{\partial\left\langle\bar{q}_{i}\right\rangle}{\partial t}=-4 \pi r^{2} D_{e, i} \frac{\partial c_{i}}{\partial r} \text { at } r=r_{p} \\
& \frac{\partial c_{i}}{\partial r}=0 \text { at } r=0 \\
& C_{i}=c_{i} \text { at } r=r_{p}
\end{aligned}
$$

where $\varepsilon_{p}$ is the intraparticle porosity, $c_{i}$ is the particle fluid phase concentration, $q_{i}$ is the particle adsorbed phase concentration, $D_{e, i}$ is the effective pore diffusivity, and $r_{p}$ is the particle radius. Equations A.1 and A.2 are spatially discretized using a backward finite difference scheme, with $N_{x}$ discretizations in the axial direction, and $N_{r}$ discretizations in the radial direction. The discretized column conservation equation combined with the boundary condition given in $A .2 b$ is: 
$\frac{\partial C_{i}^{j}}{\partial t}=-\frac{3(1-\varepsilon) D_{e, i}}{r_{p} \varepsilon} \frac{c_{i}^{j}-c_{i-1}^{j}}{\Delta r}+\frac{u}{\varepsilon} \frac{C_{i}^{j}-C_{i}^{j-1}}{\Delta x}$

where $\Delta r$ is $r_{p} / N_{r}, \Delta x$ is $L / N_{x}$, and $i$ and $j$ mark the radial and axial discretizations, respectively. This equation is applied to every component of interest in the system. For the rate equation discretization, it is convenient to first define the discretized second derivative in A.2, which assumes $D_{e, i}$ is not a function of $r$ :

$\frac{\partial}{\partial r}\left(r^{2} \frac{\partial c_{i}}{\partial r}\right)=\frac{\left(\frac{r_{i+1}+r_{i}}{2}\right)^{2} \frac{c_{i+1}-c_{i}}{\Delta r}-\left(\frac{r_{i-1}+r_{i}}{2}\right)^{2} \frac{c_{i}-c_{i-1}}{\Delta r}}{\Delta r}$

The term $r_{i \pm 1}=r_{i} \pm \Delta r$, thus:

$\frac{\partial}{\partial r}\left(r^{2} \frac{\partial c_{i}}{\partial r}\right)=\frac{\left(r_{i}+\frac{\Delta r}{2}\right)^{2}\left(c_{i+1}-c_{i}\right)+\left(r_{i}-\frac{\Delta r}{2}\right)\left(c_{i-1}-c_{i}\right)}{\Delta r^{2}}$

This is then plugged into A.2 in order to find the desired final form:

$$
\frac{\partial c_{i}}{\partial t}=\frac{D_{e, i}\left[\left(1+\frac{\Delta r}{2 r_{i}}\right)^{2}\left(c_{i+1}-c_{i}\right)+\left(1-\frac{\Delta r}{2 r_{i}}\right)^{2}\left(c_{i-1}-c_{i}\right)\right]}{\varepsilon_{p} \Delta r^{2}}-\frac{1}{\varepsilon_{p}} \frac{\partial q_{i}}{\partial t}
$$

Finally, the discretized equations, A.3 and A.6, require an assumed isotherm model in order to calculate the adsorbed phase time derivatives. This is done by implementing a full kinetic form of the various isotherm models.

For the full kinetic isotherm form, an isotherm model is re-derived without the assumption of steady-state equilibrium. For example, the Langmuir isotherm form of this is given by:

$$
\frac{\partial q_{i}}{\partial t}=k_{i}\left[c_{i}\left(q_{m, i}-\sum_{j} q_{j}\right)-\frac{q_{i}}{K_{L, i}}\right]
$$


where $k_{i}$ is an adsorption rate constant, and other parameters are defined the same as in the Langmuir isotherm. The analogous SMA treatment is given by:

$$
\frac{\partial q_{i}}{\partial t}=k_{i}\left[\left(q_{0}-\sum_{j}\left(z_{j}+\sigma_{j}\right) q_{j}\right)^{z_{i}} c_{i}-\frac{q_{i}\left(C_{N a^{+}}\right)^{z_{i}}}{K_{e, i}}\right]
$$

where, again, $k_{i}$ is an adsorption rate constant and other parameters are defined the same as in the SMA isotherm. This treatment affords an extra variable, the adsorption rate constant, which is used to impart a kinetic resistance to binding, in addition to the mass transfer resistance built into the discretization. Should $k_{i}$ be set high enough, the result would assume that mass transfer is fully controlling. Thus, there is a maximum value for $k_{i}$, beyond which the model prediction will no longer change. 


\section{Appendix B: Two-Component Adsorption Isotherms of Antibody Monomers}

The primary work presented in this dissertation deals with the two-component adsorption of a monoclonal antibody and its soluble dimer. However, in pursuit of more fully explaining the behavior involved in the monomer-dimer exchange, two-component monomer-monomer isotherms were also measured for different pairs of antibodies. This was done with the hope of finding conditions where selectivity could be found to vary with salt concentration, as it does for the monomer-dimer system. Previous work by Pérez-Almodóvar et al. (2012) had investigated the multi-component adsorption of two antibodies on macroporous and polymergrafted cation exchange resins, but the antibodies used in that work had substantially different isotherm behavior, leading to predictably high selectivities.

Figure B.1 shows the protein retention factor due to binding vs. the modifier concentration at elution from linear gradient elution experiments for mAbs A - D on Nuvia HR-S, as described in Section 3.2.4. MAb B elutes substantially earlier than the others, followed by $\mathrm{mAb} C$, and then mAbs $A$ and $D$ both eluting at similarly higher salt concentrations. Interestingly, the retention curves for mAbs $A$ and $D$ appear to cross, with mAb $D$ being less retained at higher salt concentrations, but more retained at low salt concentrations. For the two-component isotherms, an analytical technique is necessary to distinguish the individual components. From Fig. 5.1b showing the analytical CEX curve of the four antibodies, it is apparent that mAbs B and C cannot be analyzed together, as they elute at the same salt concentration. Based on the retention curves of Fig. B.1 and the analytical CEX, mAbs $A, B$, and D were chosen for isotherm measurements. This was done in order to have mAbs with substantially different retention behavior ( $B$ and $D)$, as well as very similar retention behavior ( $A$ and $D)$.

Figure B.2 shows the single component adsorption isotherms for mAbs A, B, and D on Nuvia HR$S$ at two different salt concentrations, using the protocol described in Section 3.2.9. As with the LGE dilute isotherm limit, mAb B is the least strongly adsorbed species at both 20 and $80 \mathrm{mM}$ $\mathrm{Na}^{+}$. MAbs $A$ and $D$ exhibit similar behaviors and capacities at $20 \mathrm{mM} \mathrm{Na}^{+}$, and $\mathrm{mAb} \mathrm{D}$ displays slightly stronger binding and a higher capacity at $80 \mathrm{mM} \mathrm{Na}^{+}$. 


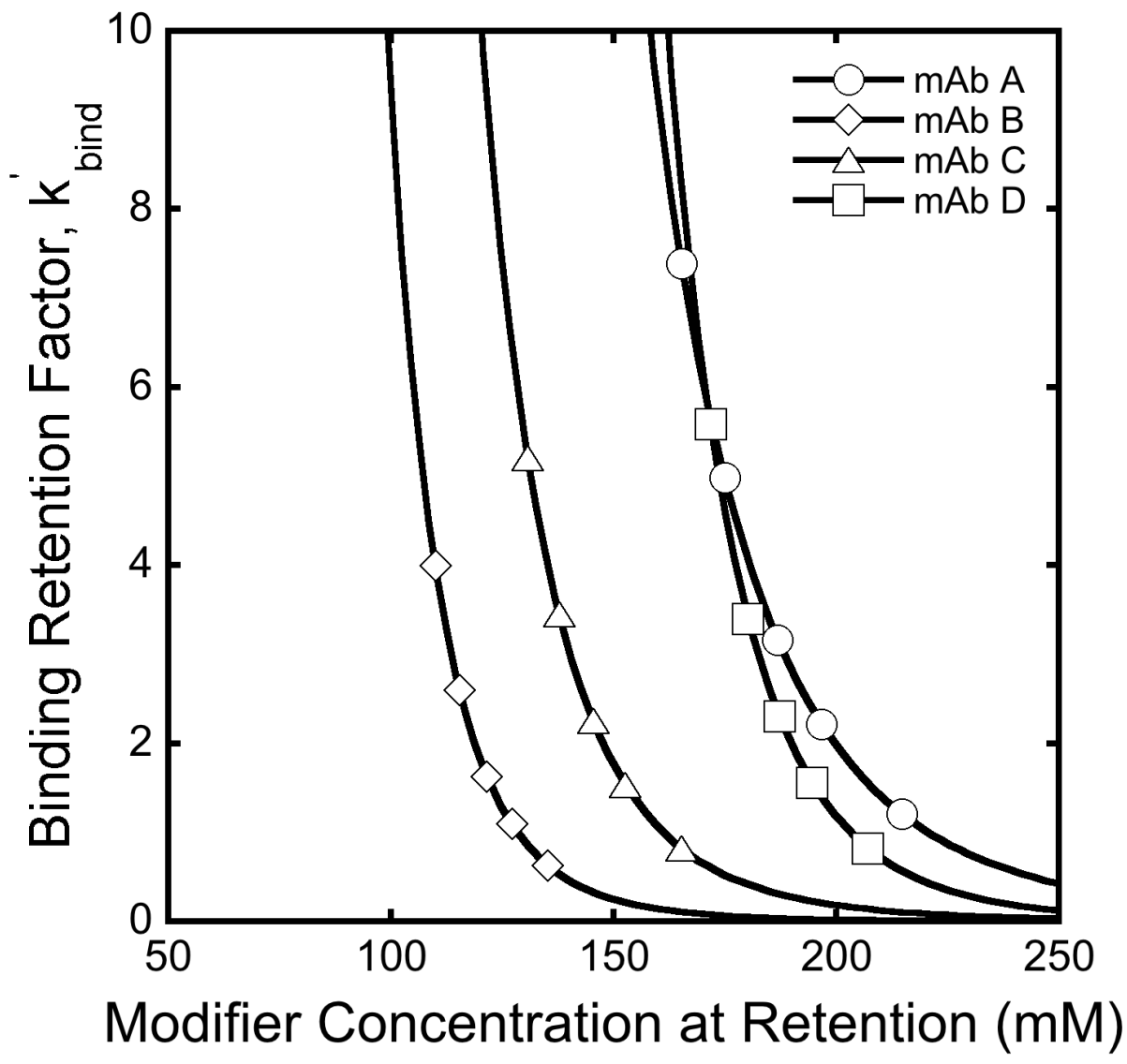

Figure B.1. Protein retention factor due to binding vs. $\mathrm{Na}^{+}$concentration at retention for mAbs A - D from linear gradient elution experiments 


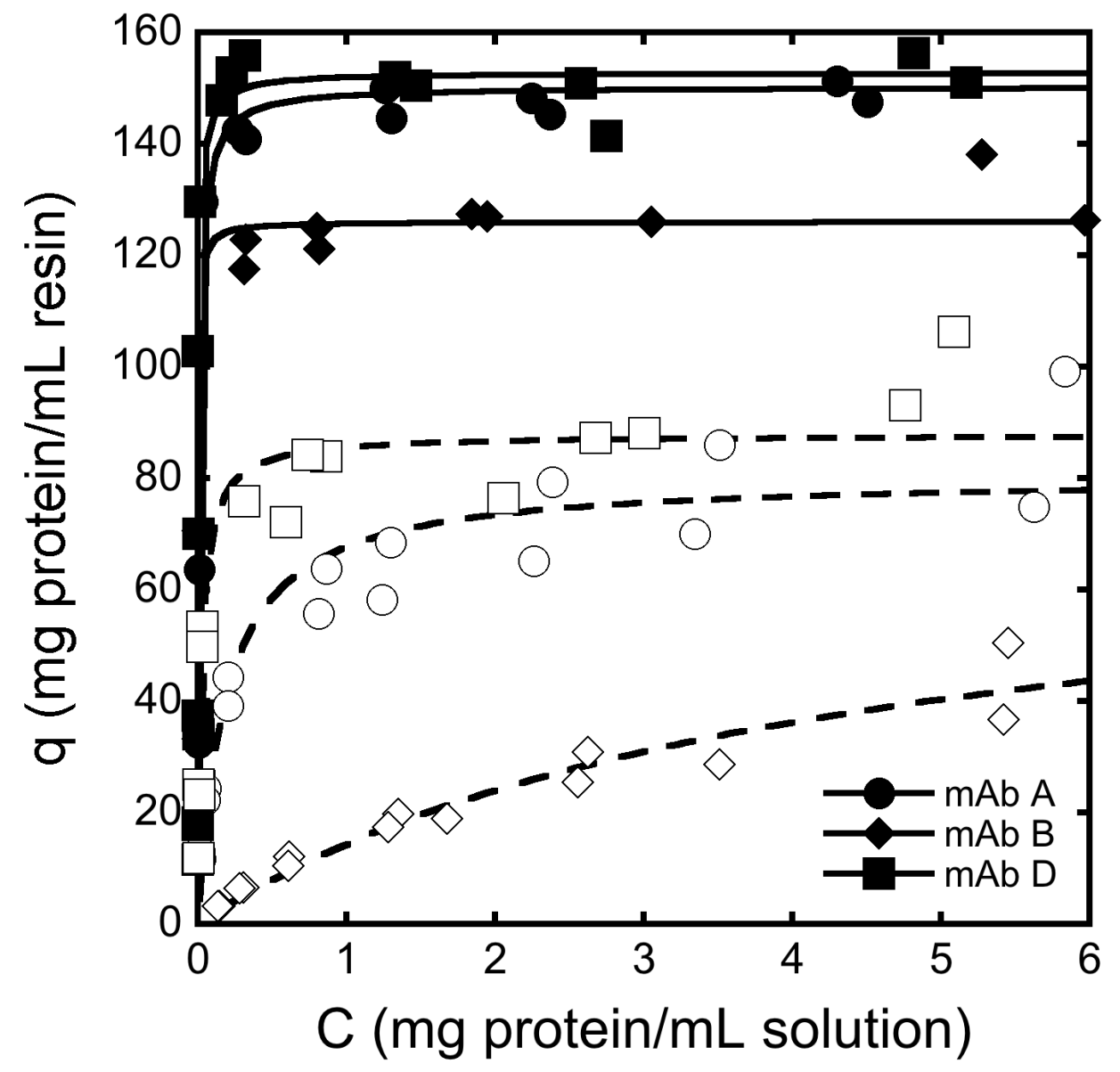

Figure B.2. Equilibrium isotherm measurements and calculated Langmuir isotherm fits for mAbs A, B, and D on Nuvia HR-S at $20 \mathrm{mM} \mathrm{Na}^{+}$(closed symbols, solid lines) and $80 \mathrm{mM} \mathrm{Na}^{+}$(open symbols, dashed lines) at $\mathrm{pH} 7.0$. 
Similar to the two-component isotherms of mAb monomer and dimer in Section 7.1, twocomponent isotherms of $m A b s B$ and $D$ and $m A b s A$ and $D$ were evaluated at different salt concentrations as described in Section 3.2.9. In these instances, HPLC CEX was used to evaluate the percentage of each component in the feed and supernatant after equilibration in order to complete the material balances. Figure B.3 shows the two-component equilibrium data in 10 $\mathrm{mM} \mathrm{Na}_{2} \mathrm{HPO}_{4}$ at $\mathrm{pH} 7.0$ with 0 and $60 \mathrm{mM}$ added $\mathrm{NaCl}$ and various ratios of $\mathrm{mAb} B$ and $\mathrm{mAb} D$. The data is also presented in tabular form in Tables B.1 and B.2. Figure B.4 shows the twocomponent equilibrium for the same conditions, but with various ratios of mAb A and mAb $D$. These data are also presented in Table B.3.

As seen from the plots, unlike the monomer and dimer system observed in the earlier Chapters, the selectivity behavior does not appear to be a strong function of salt for these monomermonomer systems. This may be expected for the mAb B and mAb D system, where mAb B has a significantly less favorable isotherm. However, the mAb A and mAb D system also displays significant selectivity, despite the two monomers having similar single component isotherm behaviors. It is uncertain why neither system seems to replicate the lack of selectivity of the monomer-dimer system at low $\mathrm{Na}^{+}$concentration, but the similar sizes of the antibodies may be an important factor. Both the adsorption isotherm experiments from Chapter 6 and the BLI experiments from Chapter 9 suggest the monomer and dimer have a similar binding orientation on the surface. Therefore, it may be that the kinetic limitations in the monomer-dimer system at low salt concentrations, and, thus, high protein loads, arise from the dimer needing to displace multiple monomers to be sterically accommodated on the surface. For the monomermonomer system when both proteins are of a comparable size, the equilibrium effects can take place without additional surface kinetic limitations, even at high protein loads. 

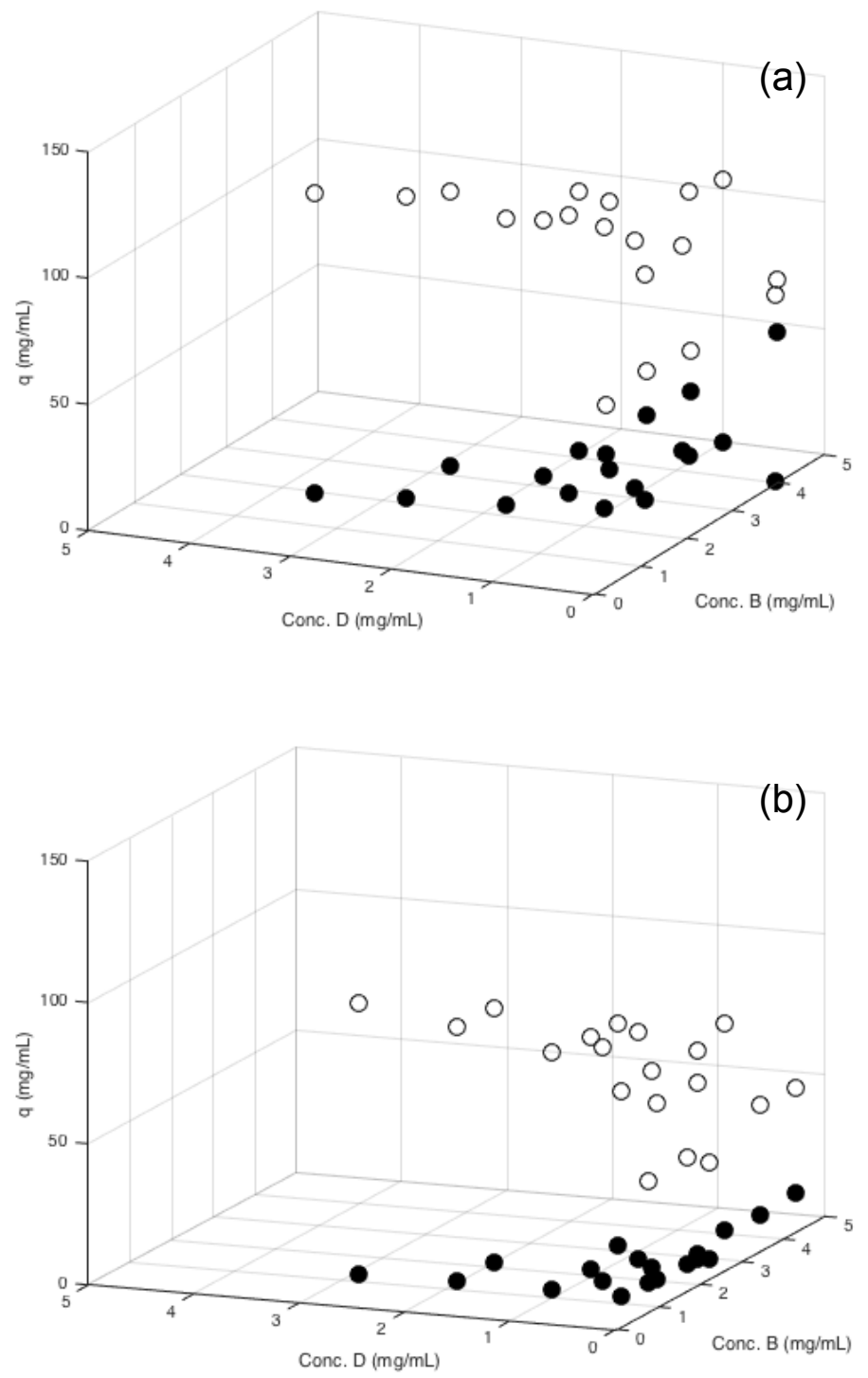

Figure B.3. Two-component adsorption equilibrium data for $m A b B$ (filled circles) and $m A b D$ (open circles) mixtures in $10 \mathrm{mM} \mathrm{Na}_{2} \mathrm{HPO}_{4}$ at pH 7.0 with (a) $0 \mathrm{mM}$ and (b) $60 \mathrm{mM} \mathrm{NaCl}$ added 


\begin{tabular}{|c|c|c|c|c|c|}
\hline $\begin{array}{c}C_{B} \\
(\mathrm{mg} / \mathrm{mL})\end{array}$ & $\begin{array}{c}C_{D} \\
(\mathrm{mg} / \mathrm{mL})\end{array}$ & $\begin{array}{c}q_{\text {total }} \\
(\mathrm{mg} / \mathrm{mL})\end{array}$ & $\begin{array}{c}q_{B} \\
(\mathrm{mg} / \mathrm{mL})\end{array}$ & $\begin{array}{c}q_{D} \\
(\mathrm{mg} / \mathrm{mL})\end{array}$ & $\alpha_{D, B}$ \\
\hline 2.66 & 1.35 & 143.7 & 20.4 & 123.3 & 11.8 \\
\hline 1.69 & 0.35 & 141.2 & 21.4 & 119.8 & 26.7 \\
\hline 0.31 & 0.02 & 123.6 & 52.1 & 71.5 & 21.8 \\
\hline 2.66 & 0.26 & 152.9 & 24.3 & 128.7 & 53.7 \\
\hline 2.14 & 0.01 & 129.1 & 56.3 & 72.8 & 188 \\
\hline 1.19 & 0.02 & 133.0 & 57.6 & 75.4 & 99.0 \\
\hline 1.71 & 1.27 & 144.0 & 21.3 & 122.7 & 7.76 \\
\hline 0.78 & 1.23 & 154.5 & 20.6 & 133.9 & 4.13 \\
\hline 0.72 & 0.23 & 161.3 & 24.8 & 136.5 & 17.3 \\
\hline 1.74 & 2.22 & 149.4 & 20.6 & 128.7 & 4.91 \\
\hline 3.60 & 0.36 & 141.4 & 18.6 & 122.8 & 66.1 \\
\hline 0.80 & 2.21 & 155.1 & 17.7 & 137.4 & 2.81 \\
\hline 4.02 & 0.03 & 73.9 & 0 & 73.9 & -- \\
\hline 0.86 & 3.15 & 148.4 & 14.8 & 133.6 & 2.48 \\
\hline 4.06 & 0.02 & 138.2 & 58.6 & 79.6 & 222 \\
\hline 2.18 & 0.83 & 148.5 & 21.4 & 127.2 & 15.6 \\
\hline 1.19 & 0.78 & 155.9 & 22.9 & 133.0 & 8.83 \\
\hline 1.20 & 0.04 & 137.2 & 24.1 & 113.1 & 147 \\
\hline 2.00 & 0.03 & 150.8 & 34.8 & 115.9 & 198 \\
\hline
\end{tabular}

Table B.1. Two-component adsorption equilibrium data for $\mathrm{mAb} B$ and $\mathrm{mAb} D$ mixtures in 10 $\mathrm{mM} \mathrm{Na}_{2} \mathrm{HPO}_{4}$ at $\mathrm{pH} 7.0$ 


\begin{tabular}{|c|c|c|c|c|c|}
\hline $\begin{array}{c}C_{B} \\
(\mathrm{mg} / \mathrm{mL})\end{array}$ & $\begin{array}{c}C_{D} \\
(\mathrm{mg} / \mathrm{mL})\end{array}$ & $\begin{array}{c}q_{\text {total }} \\
(\mathrm{mg} / \mathrm{mL})\end{array}$ & $\begin{array}{c}q_{B} \\
(\mathrm{mg} / \mathrm{mL})\end{array}$ & $\begin{array}{c}q_{D} \\
(\mathrm{mg} / \mathrm{mL})\end{array}$ & $\alpha_{D, B}$ \\
\hline 3.00 & 1.16 & 82.7 & 2.3 & 80.4 & 90.9 \\
\hline 1.97 & 0.44 & 78.7 & 4.6 & 74.1 & 72.5 \\
\hline 0.90 & 0.05 & 54.3 & 9.2 & 45.1 & 95.3 \\
\hline 2.93 & 0.39 & 76.0 & 2.0 & 74 & 284 \\
\hline 2.87 & 0.25 & 36.9 & 1.3 & 35.6 & 313 \\
\hline 1.82 & 0.04 & 54.6 & 8.3 & 46.3 & 237 \\
\hline 2.07 & 1.05 & 85.2 & 1.4 & 83.9 & 120 \\
\hline 1.13 & 1.05 & 87.1 & 1.6 & 85.5 & 57.7 \\
\hline 1.04 & 0.36 & 77.2 & 2.2 & 74.9 & 97.6 \\
\hline 2.14 & 1.99 & 90.8 & 0.4 & 90.3 & 237 \\
\hline 3.84 & 0.48 & 78.9 & 2.9 & 76.0 & 207 \\
\hline 1.20 & 1.98 & 92.1 & 1.1 & 91.0 & 48.2 \\
\hline 3.66 & 0.08 & 61.3 & 11.3 & 50.1 & 210 \\
\hline 1.26 & 2.76 & 96.6 & 0.4 & 96.2 & 104 \\
\hline 4.56 & 0.09 & 60.5 & 11.6 & 48.9 & 218 \\
\hline 2.52 & 0.78 & 84.9 & 2.1 & 82.9 & 129 \\
\hline 1.57 & 0.75 & 87.4 & 2.3 & 85.1 & 77.1 \\
\hline 1.44 & 0.18 & 73.7 & 5.6 & 68.1 & 99.4 \\
\hline 2.38 & 0.17 & 72.7 & 5.1 & 67.7 & 191 \\
\hline
\end{tabular}

Table B.2. Two-component adsorption equilibrium data for $\mathrm{mAb} B$ and $\mathrm{mAb} D$ mixtures in 10 $\mathrm{mM} \mathrm{Na}_{2} \mathrm{HPO}_{4}$ at $\mathrm{pH} 7.0$ with the addition of $60 \mathrm{mM} \mathrm{NaCl}$ 

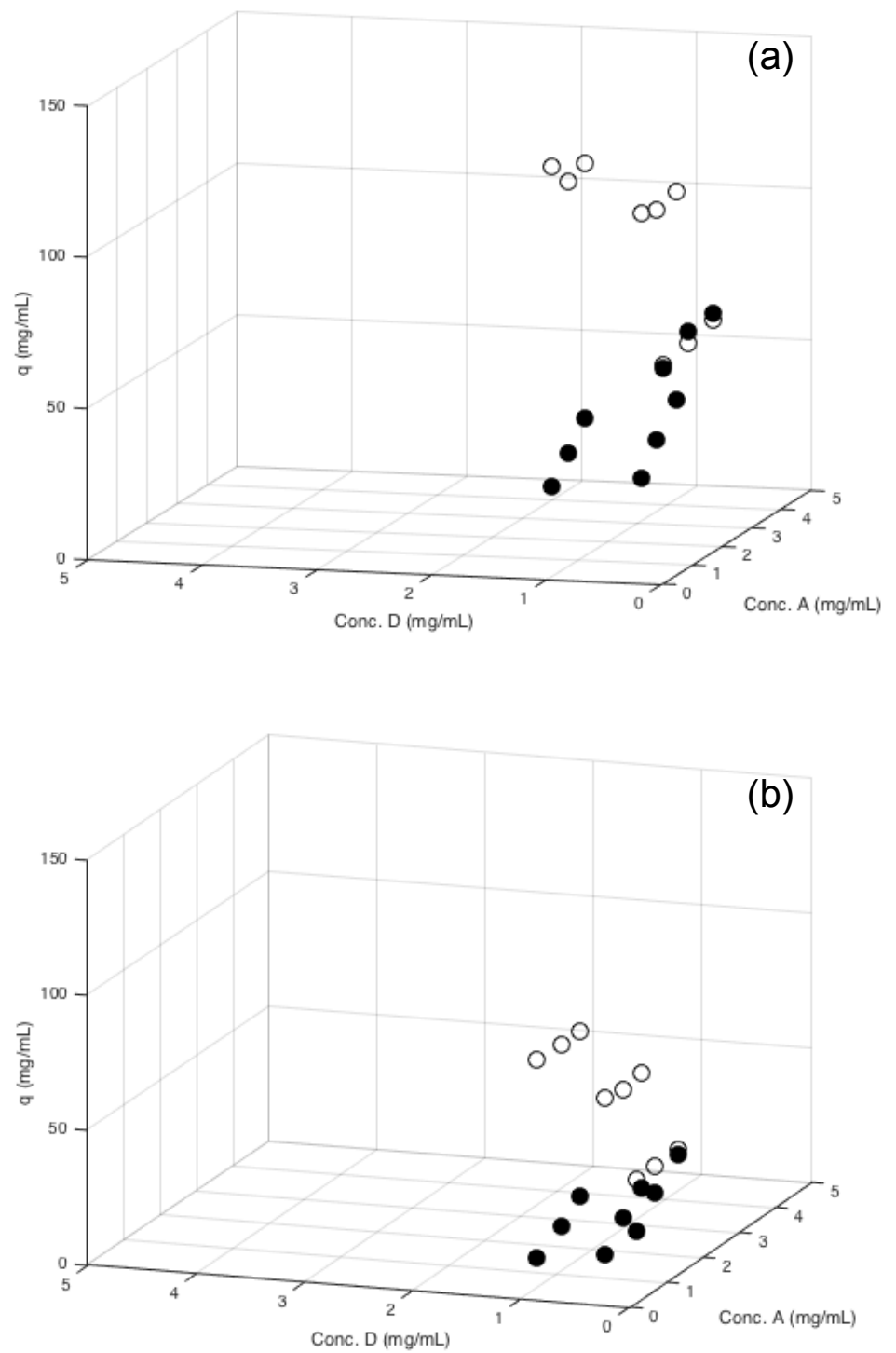

Figure B.4. Two-component adsorption equilibrium data for $\mathrm{mAb} A$ (filled circles) and $\mathrm{mAb} D$ (open circles) mixtures in $10 \mathrm{mM} \mathrm{Na}_{2} \mathrm{HPO}_{4}$ at pH 7.0 with (a) $0 \mathrm{mM}$ and (b) $60 \mathrm{mM} \mathrm{NaCl}$ added 


\begin{tabular}{|c|c|c|c|c|c|c|}
\hline $\begin{array}{l}C_{\mathrm{Na}^{+}} \\
(\mathrm{mM})\end{array}$ & $\begin{array}{c}C_{A} \\
(\mathrm{mg} / \mathrm{mL})\end{array}$ & $\begin{array}{c}C_{D} \\
(\mathrm{mg} / \mathrm{mL})\end{array}$ & $\begin{array}{c}q_{\text {total }} \\
(\mathrm{mg} / \mathrm{mL})\end{array}$ & $\begin{array}{c}q_{A} \\
(\mathrm{mg} / \mathrm{mL})\end{array}$ & $\begin{array}{c}q_{D} \\
(\mathrm{mg} / \mathrm{mL})\end{array}$ & $\alpha_{D, A}$ \\
\hline \multirow{9}{*}{0} & 2.55 & 1.33 & 157.7 & 36.8 & 120.9 & 6.30 \\
\hline & 1.52 & 0.45 & 150.9 & 37.7 & 113.2 & 10.2 \\
\hline & 0.12 & 0.02 & 142.4 & 70.5 & 71.9 & 5.69 \\
\hline & 2.41 & 0.49 & 158.8 & 45.1 & 113.7 & 12.3 \\
\hline & 1.94 & 0.05 & 152.4 & 77.2 & 75.2 & 37.6 \\
\hline & 1.01 & 0.04 & 150.5 & 77.2 & 73.3 & 23.8 \\
\hline & 1.65 & 1.25 & 152.2 & 31.2 & 121.0 & 5.13 \\
\hline & 0.78 & 1.17 & 156.4 & 25.3 & 131.3 & 3.47 \\
\hline & 0.67 & 0.35 & 148.5 & 30.5 & 118.0 & 7.34 \\
\hline \multirow{9}{*}{60} & 1.56 & 1.24 & 92.1 & 15.6 & 76.5 & 6.15 \\
\hline & 0.96 & 0.54 & 83.1 & 17.8 & 65.4 & 6.57 \\
\hline & 0.30 & 0.09 & 66.9 & 23.8 & 43.1 & 6.11 \\
\hline & 1.44 & 0.60 & 87.1 & 22.5 & 64.6 & 6.87 \\
\hline & 1.22 & 0.16 & 79.6 & 38.7 & 40.9 & 7.89 \\
\hline & 0.74 & 0.13 & 73.1 & 31.6 & 41.5 & 7.45 \\
\hline & 1.05 & 1.14 & 90.8 & 11.9 & 78.9 & 6.08 \\
\hline & 0.56 & 1.14 & 87.5 & 6.8 & 80.7 & 5.83 \\
\hline & 0.48 & 0.47 & 80.8 & 11.6 & 69.2 & 6.13 \\
\hline
\end{tabular}

Table B.3. Two-component adsorption equilibrium data for $\mathrm{mAb} A$ and $\mathrm{mAb} D$ mixtures in 10 $\mathrm{mM} \mathrm{Na}_{2} \mathrm{HPO}_{4}$ at $\mathrm{pH} 7.0$ with and without the addition of $60 \mathrm{mM} \mathrm{NaCl}$ 